\title{
Danish activities concerning noise in the environment (A)
}

\author{
Ingerslev, Fritz
}

\section{Published in:}

Acoustical Society of America. Journal

Link to article, DOI:

10.1121/1.2019901

Publication date:

1982

\section{Document Version}

Publisher's PDF, also known as Version of record

Link back to DTU Orbit

Citation (APA):

Ingerslev, F. (1982). Danish activities concerning noise in the environment (A). Acoustical Society of America. Journal, 72(S1), S45-S46. https://doi.org/10.1121/1.2019901

\section{General rights}

Copyright and moral rights for the publications made accessible in the public portal are retained by the authors and/or other copyright owners and it is a condition of accessing publications that users recognise and abide by the legal requirements associated with these rights.

- Users may download and print one copy of any publication from the public portal for the purpose of private study or research.

- You may not further distribute the material or use it for any profit-making activity or commercial gain

- You may freely distribute the URL identifying the publication in the public portal

If you believe that this document breaches copyright please contact us providing details, and we will remove access to the work immediately and investigate your claim. 


\title{
The 104th Meeting of the Acoustical Society of America
}

\author{
Sheraton Twin Towers • Orlando, Florida • 8-12 November 1982
}

\section{TUESDAY MORNING, 9 NOVEMBER 1982}

BROWARD AND PALM BEACH ROOMS, 8:00 TO 10:15 A.M.

\section{Session A. Underwater Acoustics I: The Impact of Satellite and Aerial Remote Sensing on the Study of Ocean Acoustics}

\author{
Paul D. Scully-Power, Chairman \\ Naval Underwater Systems Center, New London, Connecticut 06320 \\ Chairman's Introduction-8;00 \\ Invited Papers
}

8:05

A1. Ocean features observed from manned spacecraft that influence acoustics in the upper ocean. Robert E. Stevenson (Office of Naval Research, Scripps Institution of Oceanography, A-010, La Jolla, CA 92093)

Ocean fronts, eddies, and internal waves have been observed, photographed, and imaged by synthetic aperture radar during the first four orbital missions of the space shuttle, Columbia. Supporting data show that the visual features correspond to thermal discontinuities, have significant vertical extensions, act as barriers to underwater acoustics, and can be detected by synthetic aperture radar regardless of sky conditions or time of day. Although one thinks of these sea-surface manifestations as having a certain ubiquity, presently available information seems to show that they are concentrated bath generically and geographically. This concept is to be addressed from Columbia and Challenger during 1983.

$8: 25$

A2. Connection between acoustic and electromagnetic sea-surface backscatter. Suzanne T. McDaniel (Applied Research Laboratory, The Pennsylvania State University, University Park, PA 16802)

The performance of active high-frequency acoustic systems operating under water is, in many cases, limited by backscattering from the sea surface. The strength of surface reverberation has been experimentally found to depend on the speed of the wind driving the ocean waves, while the Doppler spread of the scattered signals has been related to the waveheight. Electromagnetic sea-surface backscatter may be used to remotely sense the ocean surface and, hence, provides a means of empirically predicting the properties of acoustic reverberation. We investigate the relationship between acoustic and electromagnetic surface backscatter. The physical mechanisms responsible for acoustic backscatter are discussed and the extent to which the relevant oceanic parameters may be extracted from electromagnetic backscatter data is assessed. It is concluded that electromagnetic remote sensing of the ocean surface provides a viable means of predicting both the strength and Doppler characteristics of underwater reverberation.

\section{8:45}

A3. Prediction of ambient noise in the deep ocean from surface wind data obtained via satellite. $D$. Shonting (Naval Underwater Systems Center, Newport Laboratory, Newport, RI 02840)

Open ocean measurements at both shallow and deep depths show that ambient acoustic noise above 10-15 $\mathrm{kHz}$ increases nearly linearly as the log of the wind speed. This relationship is studied under a variety of wind/ sea conditions at 15,20 , and $25 \mathrm{kHz}$ bands using a newly developed self-contained ambient noise recorder (SCANR). Assessment is made of possible ambient noise prediction from wind speed data obtained from a satellite mounted microwave scatterometer. 
A4. Satellite remote sensing and underwater acoustics: What you see is not necessarily what you get. Robert F. Henrick and Charles L. Johnson (Johns Hopkins University, Applied Physics Laboratory, Johns Hopkins Road, Laurel, MD 20810)

Continuing efforts in satellite systems and analysis methodology may soon result in routine production of ocean weather maps for substantial regions of the world's oceans. Such maps might illustrate positions of such mesoscale features as ocean fronts and eddies and be available to the acoustician on a routine basis. (In fact such products are presently available for the Gulf Stream region.) However, the use of such maps without guidance as to the acoustic significance of these features may have limited utility to an acoustician deciding on the deployment of an acoustic system, and may indeed serve to mislead. A front or eddy that may be clearly visible to a satellite sensor may not be acoustically significant to the system under consideration. Even if the mesoscale feature has acoustic impact, the surface expression of the feature may not coincide with the acoustically important portion of the feature. Alternately, acoustically relevant mesoscale anomalies may not be visible to a satellite sensor. To illustrate these cautions, oceanographic data taken by the U.S. Naval Oceanographic Office Ocean Measurements Program in the Northeast Atlantic and Norwegian Sea are utilized in conjunction with numerical acoustic propagation simulations. This region is typical of areas where significant mesoscale activity occurs and oceanographic sampling is sparse, so that satellite remote sensing may be very useful in predicting acoustic system performance. However, examples presented illustrate that significant work must be done before a useful satellite oceanographic product can be turned into a useful acoustic product. [Work supported by the U.S. Naval Oceanographic Office.]

9:25

A5. SAW's, the connection between space and hydrophones. Paul A. Nysen (Crystal Technology Inc., Palo Alto, CA 94303)

SAW (Surface Acoustic Wave) devices are a new class of signal processing and signal generating elements now in use with radar and communication systems. These devices can be made as filters, delay lines, or resonators which are small, rugged, and inexpensive. This paper discusses the mechanical and thermal sensitivities of SAW's with a view towards using these properties to sense temperature, pressure, and sound in water. Various modulation schemes are examined. Finally a buoy system is explored, involving a gauge string of SAW hydrophones/temperature/pressure sensors which can be enabled and interrogated from a space platform.

\section{Contributed Papers}

\section{$9: 45$}

A6. The use of satellite data for the detection and estimation of large acoustic anomalies caused by ocean currents. James $V$. White and Robert F. Brammer (The Analytic Sciences Corporation, 1 Jacob Way, Reading, MA 01867)

The Seasat-A satellite carried a radar altimeter and a microwave scatterometer that provided nearly global data sets on sea surface height, significant wave height, and sea-surface wind velocity. These data, and similar data from future satellite missions, can be used to estimate ocean parameters that effect underwater sound generation, propogation, and surface scattering. This paper describes a matched-filter technique for using altimeter data to detect, locate, and estimate the sizes of large coherent acoustic anomalies caused by mesoscale $(50 \mathrm{~km}-500 \mathrm{~km})$ rotating current rings. These ring currents are spawned by the Gulf Stream, have lifetimes ranging from several months to two years, and produce characteristic sea-surface height signatures in the altimeter data. A technique for processing the altimeter data to estimate sound-speed profiles in cold-core current rings is discussed, and results for representative ring currents are presented.

\section{0:00}

A7. Satellite detection of anomalies in ocean ambient noise? Calvin R. Dunlap and Glenn H. Jung (Oceanography Department, Naval Postgraduate School, Monterey, CA 93940

ASTREX (Acoustic Storm Response Experiment) observations made in mid-November 1980, along a flight line from Cape Mendocino, California, toward the central Gulf of Alaska, included concurrent airborne expendable bathythermograph (AXBT) measurements of surface and subsurface temperatures and ambient noise measurements $(1-2000 \mathrm{~Hz})$ using sonobuoys, with hydrophones deployed at $400 \mathrm{ft}(122 \mathrm{~m})$. NOAA- 6 satellite infrared observations also were available. It is shown that anomalous (low) ambient noise values, measured at 122 meters and frequencies 500 $2000 \mathrm{~Hz}$, appear to be associated with a warm-core eddy extending from the surface to depths greater than $250 \mathrm{~m}$. This thermal feature is shown clearly at the sea surface in a satellite infrared photograph. It appears that the warm-core eddy had refracted ambient noise away from the hydrophone, since ambient noise levels are several decibels higher outside the eddy region. It is suggested that careful studies are warranted of relations between ambient noise, thermal structure, and surface temperature features detectable by satellite, exploring the possibility of using satellites in some circumstances to indicate subsurface ambient noise anomalies. 


\title{
Session B. Architectural Acoustics I: The Technical Committee on Architectural Acoustics V. O. Knudsen Distinguished Lecture
}

\author{
Ludwig W. Sepmeyer, Chairman \\ 1862 Comstock Avenue, Los Angeles, California 90025 \\ Chairman's Introduction-8:30
}

Invited Paper

$8: 35$

B1. Complexity and contradiction in proscenium theater design-The last one hundred years. George C. Izenour (Izenour Associates, New Haven, CT 06515)

Proscenium theater design, rooted in Baroque tradition of court theater design and Beaux Arts architectural design achieved apogee in Opera de Paris (1875). Bayreuth Festpielhaus (1876) touched off a controversy in theater design among architecture, engineering, and artistic style that persists to this day. Opera de Paris was the last theater of historical importance where the architect alone made all crucial design decisions. Bayreuth Festpielhaus was the first theater of historical significance where the architect shared responsibility for design and technology with a theater consultant. At the time there was no such thing as an acoustical consultant. The ensuing century (1880-1980) changed everything. The industrial revolution changed structure and added mechanical and electrical engineering to the building process. Sabine provided the method for acoustical predesign of interior spaces. Post W.W. II computer revolution provided sophistication of operation. Mechanized transportation changed forever the relation of theater buildings to the overall fabric of urban design. These disparate disciplines have each contributed to the complexity of design and execution of theater buildings and the still unresolved artistic styles have all together become the modus operandi of contemporary theater design. It is for reasons both of a program dictating multiple use and the sheer magnitude of the engineering solutions resulting therefrom that apogee of proscenium theater design for our time will have been consumated in Teatro Teresa Carrena to be opened in Caracas, Venezuela (1983).

\section{Contributed Papers}

$9: 35$

B2. Acoustical modeling of 'Troy Music Hall. D. P. Ayyappana) (School of Architecture, Rensselaer Polytechnic Institute, Troy, NY 1218I)

An acoustical model built to a scale of 1:16 was utilized in evaluating the acoustics of the Troy Music Hall in Troy, New York. The model was modified into different configurations to identify the specific physical characteristics of the hall that might aid the acoustical qualities of the hall. Technical limits and usefuiness of an acoustical model to determine the probable acoustical qualities of concert halls at an early stage were evaluated by comparing the objective qualities in the model and in its prototype. The initial assumption involves the accepted high quality of Troy Music Hall. It was found that an acoustical model is an effective research tool, within limitations, in probing the early energy components. The objective data obtained from the model studies coincide, in general, with the subjective qualities found in the hall. The model study shows that the first balcony in the Troy Music Hall receives an equal distribution of lateral and non-lateral energies in the first $80 \mathrm{~ms}$. 'P Presently employed as Consultant in Theater Acoustics with Pelton/Blum, Inc., 1801 North Lamar, Dallas, TX 75202.

\section{9:50}

B3. A sonic tour of symphonic concert halls. J. Robert Ashley (Department of Electrical Engineering, University South Florida, Tampa, FL 33620)
The Koss CM-1030 speakers designed by the author will be described to justify their choice for the demonstration at the 104th meeting. The 100 -liter bass section was synthesized to a $30-\mathrm{Hz}$ BB4 alignment. Dual closed-box $800 \mathrm{ml}$ mid-range systems are crossed over at $300 \mathrm{~Hz}$. Dome tweeters cover the spectral ranges of 2 to 6 to $15 \mathrm{kHz}$. These speakers have much lower intermodulation distortion than most commercially marketed speakers. Telarc $\left.{ }^{(}\right)$digitally mastered recordings will be used for most of the sonic examples. These recordings have been made with three omnidirectional microphones and little equalization. A recording from the current best concert hall in the world, Powell Hall in St. Louis, will illustrate the accuracy of the recording and playback process. Another good hall, Symphony Hall in Boston, will show why the musicians prefer halls such as these. The cold, steely string quality of many modern halls will be sonically illustrated by the Atlanta Symphony. Slides will illustrate the physical causes of sonic differences.

\section{0:05}

B4. Effects of auditorium acoustics on musical performance. John Charles Cox (International Jensen, Inc., 4309 Transworld Road, Schiller Park, IL 60176)

The echo perception thresholds of listeners and performers are compared. Performer's minimum detectabie delay (MDD) and maximum tolerable delay (MTD) are presented. The effects of several auditoria on the performers of music are shown. 


\title{
Session C. Noise I and CCEA: The National Noise Control Program: Needs and Strategies
}

\author{
Henning E. von Gierke, Chairman
}

Aerospace Medical Research Laboratory, AFAMRL/BB, Wright-Patterson AFB, Ohio 45433

\author{
Chairman's Introduction-8:30
}

Invited Papers

C1. Transportation noise: The federal viewpoint. John E. Wesler (Office of Environment and Energy, Federal Aviation Administration, Washington, DC 20553)

Over the past year and one-half, the Federal program for transportation noise control has undergone a number of changes, both in direct and overall policy. Transportation noise control rernains an important priority, especially since public reaction to transportation noise severely constrains the efficient growth of a national transportation system needed to meet forecast demands for the movement of people and goods. In some cases, noise is forcing reductions in system capacity, directly in the face of demands for system expansion. This paper will review recent changes in Federal noise control programs and policies, and will attempt a brave look into the future of those activities.

\section{9:10}

C2. Proposed noise strategy for commercial aircraft. Richard J. Linn (Office of Technological Development, American Airlines; P.O. Box 61616, Dallas-Ft. Worth Airport, TX 75261)

Since recent court rulings have given the local airport proprietor more responsibility in reducing noise in airport communities without being discriminatory to any one airline, and in light of the continued difficulty in achieving federal pre-emption, this paper will present a strategy for achieving meaningful noise reduction without undo legislation by governing bodies.

\section{9:40}

C3. The national noise control program: needs and strategies; motor vehicle noise: the industry viewpoint. Paul P. Pataky (MVMA Vehicle Noise Committee, 300 New Center Bldg., Detroit, MI 48202)

This presentation briefly describes the history of the motor vehicle industry's involvement in the understanding and control of transportation noise and, specifically, the impact of motor vehicle noise on overall community noise levels. Recognizing the need for valid noise regulations, the presentation then discusses industry's requirement for harmonized vehicle noise standards and test procedures in order to facilitate compliance to those regulations in the most cost-effective manner possible. The paper then presents industry's recommendations for future strategies that can be implemented to meet the objective of achieving a quieter environment at the least cost to society. These strategies include the use of computer mathematical models to determine the environmental impact of new vehicle noise regulations and to assess other, perhaps more viable and more cost-effective alternatives to vehicle noise regulations. Also discussed are strategies that can be pursued jointly by industry, the voluntary standards associations and the motor vehicle regulators to achieve harmonization of motor vehicle noise standards and compliance test procedures, not only on a national, but on a worldwide basis.

\section{0:10}

C4. Noise, land use planning and the HUD noise policy. Richard H. Brown (Office of Environment and Energy, Dept. of Housing and Urban Development, Washington, DC 20410)

As opportunities for additional significant reductions in major noise sources decrease, land use planning remains one of the few ways to prevent noise problems from developing. The HUD noise policy specifically encourages communities to consider noise in their land use planning. It is not, however, the total answer by any means. There will always be situations where other local priorities must take precedence, particularly in urban areas. Noise attenuation measures will continue to be important and a crucial part of the HUD noise policy. Lacking any specific legislative authority on general land use issues, the Federal role can only be to encourage local governments to consider noise in their land use planning process and to provide information. 
C5. The national noise control program: Needs and strategies-State and local perspectives. Jesse O. Borthwick (National Association of Noise Control Officials, P.O. Box 2618, Fort Walton Beach, FL 32549)

On October 1, 1982, the U.S. Environmental Protection Agency's noise control program came to an end. The program was phased out following a determination by the Reagan Administration that, "the benefits of noise control can be adequately carried out at the State and local level without the presence of a federal program." This decision to defederalize noise control has raised many questions concerning the further roles and needs of state and local noise control programs, such as: What actions will the states and cities take in, the area of new product noise regulation, and how will these actions affect industry? Where will noise control officials be able to turn for technical and financial assistance? The author will discuss these and other questions currently confronting state and local noise control professionals.

\section{1:20}

C6. European commissions' noise abatement action program-A global approach on an international level. J. M. Junger (Environment Commission of the European Communities, Rue de la Loi 200, B1049 Brussels, Belgium|

The purpose of this paper is to indicate the role of the EEC's Action Programme for Noise Abatement, to present its evolution and to place it in the context of the European Community. It is also intended more particularly to discuss the interaction between this programme and (1) the national, regional, or local programs; (2) the global economic interests, which result from the free circulation of goods and a reduced distortion of competition; (3) energy saving. In conclusion, it is attempted to point at some criteria which might possibly constitute a basis for a coordinated noise abatement policy at an international level. The key word will be "harmonization" which must be understood as an optimal relationship between different components of a whole.

\section{1:50}

C7. Voluntary noise standards to meet national needs. Kenneth McK. Eldred (KEE, P.O. Box 1037, Concord, MA 01742)

The apparent termination of a federal noise control program in the Environmental Protection Agency removes a potential for a coordinated Fedéral leadership in the field of environmental noise in the USA, except for the areas in which authority is vested in other Federal agencies, such as the Department of Transportation, Defense, Labor, and Housing and Urban Development. At the same time the needs for control of various aspects of environmental noise persist both here and throughout the world. It may be anticipated that many states and localities will attempt to meet some of these needs through regulations and other activities. The the extent that these regulations and controls require measurement, harmonization of noise scales and measurement procedures is required, if the result is to be nonchaotic. This paper gives a brief overview of the extent of the potential impacts of various parts of the environmental noise problem in terms of sound exposure and population exposed. It then addresses the potential roles and interaction between sound and operating controls and summarizes their current status with respect to the various parts of the environmental noise problem. From this summary it deduces the needs for measurement procedures and their harmonization, and explores the possible roles of the voluntary standards sector in fulfilling these needs.

12:20 to 12:50

Panel Discussion

"Where Do We Go From Here" 


\title{
Session D. Physiological Acoustics I: Peripheral Acoustics and Physiology
}

\author{
John F. Brugge, Chairman \\ Department of Neurophysiology, 627 Waisman Center, University of Wisconsin, Madison. Wisconsin 53706
}

Chairman's Introduction- $-\mathbf{8 : 3 0}$

\section{Contributed Papers}

$8: 35$

D1. The influence of the sound level of a steady-state broadband noise on the temporal response of the acoustic reflex. Nicole Lalande and Raymond Hétu (Ecole d'orthophonie et d'audiologie, Université de Montréal, C. P. 6128, Montréal, Québec, Canada H3C 3J7)

The influence of the sound level of a steady-state pink noise on the short- and the long-term response of the contralateral acoustic reflex was investigated with nine adult subjects. Three noise levels were selected $\left(L_{\rho A}\right.$ of 95, 100, and 1 10) for a duration of $16 \mathrm{~min}$ (or $8 \mathrm{~min}$ occasionally). Based on previous data [R. H. Wilson et al., J. Acoust. Soc. Am. 64, 782-79] (1978)], it was hypothesized that when the reflex activity is expressed in absolute value of change in acoustic susceptance, the reflex decay is the same whatever the intensity of the noise; expressed in relative value of percentage of change in susceptance, the reflex adaptation is greater for a low level of noise than for higher ones. Results clearly confirmed the above hypothesis. These findings explain the conflicting results previousiy obtained in terms of the way data are analyzed. Practical implications concerning the protection afforded by the acoustic reflex under such noise exposures will be discussed.

\section{8:50}

D2. The importance of external and middle ear contributiens to bone conduction in man. S. Gatehouse (MRC Institute of Hearing ResearchScottish Section, Southern General Hospital, Glasgow, Scotland)

Differences between air conduction (a-c) and bone conduction (b-c) thresholds are commonly used to determine the presence and magnitude of a middle ear abnormality in the human audiotory system, making the tacit assumption that $b-c$ directly stimulates the cochlea. Animal experiments have long established that b-c transmission is a complex phenomenon [J. Tonndorf, in Foundations of Modern Auditory Theory, Vol. 2 (Academic, New York, 1972)] with significant contributions from middle and external ear components. An artificial reversible middle ear abnormality may be induced in man by maintaining an air pressure in the external meatus. The shifts in a-c and b-c thresholds provide a means to evaluate the relative importance of the external and middle ear components. At the lower frequencies of 250 and $500 \mathrm{~Hz}$, these components predominate; at $500 \mathrm{~Hz}$ there is an a-c shift of $15.9 \mathrm{~dB}$ accompanied by a b-c shift of 15.8 dB. These results have important implications for the interpretation of air and bone conduction thresholds.

\section{9:05}

D3. Ear-canal resonances and the assessment of hearing thresholds at high frequencies. K. N. Stevens, S. H. Blumenthal, D. M. Green, and M. Krasner (Bolt Beranek and Newman, Inc., Cambridge, MA 02238)

Standing waves in the ear canal cause substantial difficulties in the assessment of high-frequency $(8000-20000 \mathrm{~Hz})$ hearing thresholds because of uncertainties in the specification of the acoustic stimulus. A calibration procedure is proposed for estimating the sound pressure $p_{1}$ at the inner end of the ear canal by measuring the poles of the impulse response at the entrance to the ear cunal when an acoustic source is coupled directly to the canal through a short tube. This calculation is based on the fact that the transfer function from the source to $p_{1}$ is an all-pole function. As a step towards implementation of this procedure, frequencies and bandwidths of ear-canal resonances for a number of ears have been measured. The data show that these resonances have bandwidths as small as $300 \mathrm{~Hz}$, and deviations from equal spacing that are usually no greater than $10 \%$. The pressure $p_{i}$ has been calculated from the appropriate all-pole transfer function, and shows systematic and predictable differences with measured peak sound pressures in the outer 1 to $1.5 \mathrm{~cm}$ of the ear canal, as expected on the basis of measurements with models. [Supported by a contract from NINCDS.]

\section{9:20}

D4. Electrical stimulation of the auditory nerve: Membrane models applied to the interpretation of electrophysiological and psychophysical responses. $M$. White and $M$. Merzenich (Department of Otolaryngology, UCSF, San Francisco, CA 94143)

Electrophysiological and psychophysical measures of threshold as a function of sinusoidal stimulus frequency deviate considerably from those predicted by Hill's membrane model. Using a modified Hodgkin-Huxley model, considerably better estimates of threshold were obtained over the frequency ranges investigated. The Hodgkin-Huxley model was modified by increasing the sodium inactivation rate constant, beta- $h$, by a factor of four. This model has been useful in interpreting electrophysiological and psychophysical responses to a range of pulse and sinusoidal stimuli. [Work supported by NIH.]

\section{9:35}

D5. Ear-canal acoustic emissions as frequency-specific indicators of cochlear function. Richard A. Schmiedt and Cheryl L. Addy (Department of Otolaryngology, Medical University of South Carolina, Charleston, SC 29425]

Acoustic emissions in the form of distortion products $\left(2 f_{1}-f_{2}\right)$ can be measured in the external ear canal. Previous work has shown that the nonlinearities generating these emissions reside in the cochlea and disappear with metabolic disruption. Distortion products measured in the cochlear microphonic and in the responses of auditory-nerve fibers have been shown to be generated largely at the cochlear location associated with the primary tones. If cochlear emissions behave similarly, it should be possible to generate an "acoustic-emission audiogram" with appropriately placed primary pairs; i.e., low-frequency pairs could be used to test the cochlear apex, high-frequency pairs to test the base. We have obtained acoustic-emission and whole-nerve action potential audiograms in Mongolian gerbils and cats both before and after exposure to narrowband noise sufficient to cause a temporary threshold shift. Our results indicate that emssions produced by lower-level primaries (70 $\mathrm{db}$ SPL) seem to reflect the recovery of the whole-nerve response over time better than higher-level primaries ( $80 \mathrm{~dB}$ SPL). Further, acoustic emissions can be used on a frequency-specific basis to monitor the condition of the mid and basal regions of the cochlea. [Work supported in part by a South Carolina Biomedical Research Grant.]

\section{9:50}

D6. Acoustic and auditory nerve measurements of distortion products. $P$. F. Fahey (Department of Physics, University of Scranton, Scranton, PA 
18510) and J. B. Allen (Acoustics Research Department, Bell Laboratories, Murray Hill, NJ 07974)

We have made measurements of distortion products responses in the ear canal and in the auditory nerve of the cat. The frequencies and levels of the primaries, $f_{1}$ and $f_{2}$, were varied in such a way that the frequency and level of the distortion product, as measured by the response of a neuron at its threshold, were held constant. In particular, we found, as has been previously reported by others, that the amplitude of the primaries needed to give an isoresponse was relatively independent of frequency for the $f_{2}-f_{1}$ signal and strongly frequency dependent for the $2 f_{1}-f_{2}$ signal. The level and frequency dependence of the distortion product $2 f_{1}-f_{2}$ as measured in the ear canal (which were not seen when the driver was terminated with an acoustic cavity) seem to agree with the level and frequency dependence of the distortion products that were detected by single neurons in the auditory nerve.

\section{0;05}

D7. Phase and group delay in the auditory nerve relative to the cochlear microphonic. J. B. Allen (Acoustics Research Department, Bell Laboratories, Murray Hill, NJ 07974)

We have extended our earlier measurements of cochlear phase response by measuring the round window potential phase, which is then used as a reference phase. This normalization greatly simplifies the phase and group delay because it appears to remove middle ear artifacts. After being normalized, the group delay shows a monotonically increasing delay with increasing frequency. This data seems to be inconsistent with a large class of "second filters."

10:20

D8. Extensions of Davis' hair cell model. J. B. Allen (Acoustics Research Department, Bell Laboratories, Murray Hill, NJ 07974)

By making a very simple modification to the Davis model of hair cell transduction, it is possible to accurately model neural period histogram data. The model data match the neural data over the entire range of experimental observations. Computed responses to pure tones and to tone bursts will be compared to experimental observations.

\section{$10 \cdot 35$}

D9. Coding of auditory information in pigeon vestibular nerve fibers. $\mathrm{H}$. P. Wit and J. D. Bleeker (Institute of Audiology, University Hospital, Postbox 30.001, 9700 RB Groningen, The Netherlands)

After fenestration of the lateral semicircular canal of the pigeon auditory stimuli (e.g., tone bursts) evoke phase-locked responses in the vestibular nerve. Single unit activity was measured in the ampullary branch of the vestibular nerve innervating the crista in the horizontal semicircular duct. The sound stimulus was delivered to the eardrum of the pigeon through a silastic tube. Most units show best phase lock for frequencies of about 700 Hz. Few units however seem to be "tuned" to lower frequencies. The lowest sound pressure level that has an observable influence upon the firing pattern of a unit is about $95 \mathrm{~dB} \mathrm{SPL}$ at the units best frequency. The low-frequency slope of the tuning curve for a unit (sound pressure level versus stimulus frequency for a present synchronisation criterion) is approx. $18 \mathrm{~dB} /$ oct. The high-frequency slope is $25 \mathrm{~dB} /$ oct. If the roof of the membranous ampulla wall is directly stimulated with a piezoelectric vibrator, tuning of most fibers is not different from the tuning measured with stimulation by (sound) pressure variations in the ear canal. This rules out the middle ear system of the pigeon as the primary source for the observed tuning. The threshold for observable phase lock in direct stimulation is a vibrator amplitude of $20 \mathrm{~nm}$, corresponding to a velocity of 0.1 $\mathrm{mm} / \mathrm{s}$ at $700 \mathrm{~Hz}$.

\section{$10: 50$}

D10. Effects of stimulating frequency on discharge patterns of single eighth nerve fibers in the bullfrog. Andrea L. Megela (Hunter Laboratory of Psychology, Brown University, Providence, RI 02912)
Temporal discharge patterns to tone bursts at best excitatory frequency (BEF) have been examined in single eighth nerve fibers of many vertebrates. There are few descriptions, however, of how discharge patterns may vary in response to tone bursts at other frequencies within a fiber's excitatory tuning curve. Discharge patterns of single eighth nerve fibers in the bullfrog were studied in response to tone bursts at BEF, and at those frequencies representing upper and lower flanks of the tuning curve at 10 and $20 \mathrm{~dB}$ above threshold. High-frequency-sensitive fibers innervating the basilar papilla showed no changes in their discharge patterns, analyzed as PST histograms, with stimulating frequency. For these fibers, the shape of the PST histogram at BEF provides a good prediction of the histogram shape at frequencies at the tuning curve flanks. On the other hand, the discharge patterns of low-frequency-sensitive fibers innervating the amphibian papilla varied with stimulating frequency, from a more sustained mode of firing at BEF to a more phasic mode of firing above BEF. Thus, the discharge patterns of amphibian papilla fibers show more variability with stimulating frequency than do those of basilar papilla fibers. [Supported by NIH grant NS09244 to R. R. Capranica.]

\section{1:05}

D11. Responses of cerebellar units to acoustic stimuli in the CF-FM bats, Pteronotus parnellii parnellii and Pteronotus parnellii rubiginosus. Philip H.-S. Jen, Xinde Sun, and Tsutomu Kamada (Division of Biological Sciences, University of Missouri, Columbia, MO 65211)

Single units which faithfully fired action potentials to pure tones (35 $\mathrm{ms}$ in duration and $0.5 \mathrm{~ms}$ rise and decay times) could be recorded from the cerebellar vermis and hemispheres of the CF-FM bats, Pteronotus parnellii parnellii and Pteronotus parnellii rubiginosus. Most units were recorded at a depth less than $1000 \mu \mathrm{m}$ from the brain surface and they fired less than five impulses to acoustic stimuli. Only a few units fired tonically to acoustic stimuli. Off responses could be recorded from those units with best frequencies tuned at around $61 \mathrm{kHz}$ which is the predominant CF component of the bat's echolocative signals and Doppler-shifted echoes. Response latencies of these units were between 1.5 and $27 \mathrm{~ms}$ and minimum thresholds were between 2 and $83.5 \mathrm{~dB} \mathrm{SPL}$. While most of the threshold curves of these units were either broad or irregular, those curves with best frequencies tuned at around $61 \mathrm{kHz}$ were extremely sharp. The highest $Q_{10}-\mathrm{dB}$ value obtained from the sharply tuned threshold curves was 153 . These data indicate that auditory specialization for processing of species-specific echolocative signals also exists in the cerebellum of the CF-FM bat. [Work supported by NSF BNS 80-07348 and USPH 1-K04NS-00433-03 to P. Jen.]

\section{1:20}

D12. Auditory physiological properties of the units in the cerebellum of the FM bats, Eptesicus fuscus and Myotis lucifugus. Philip H.-S. Jen, Tsutomu Kamada, and Xinde Sun (Division of Biological Sciences, University of Missouri, Columbia, MO 65211)

Response properties of cerebellar units in the FM bats, Eptesicus fuscus and Myotis lucifugus were studied under free-field and closed-system sound stimulation. Units responding to sound stimuli could be isolated from a rather large area of cerebellar vermis and the cerebellar hemispheres. Only a few units fired tonically while most discharged between one and five impulses during acoustic stimulation. Response latencies ranged between 2.97 and $68 \mathrm{~ms}$ with most between 8 and $14 \mathrm{~ms}$. Threshold curves were broadly tuned with a triangular or irregular shape. Similar to units in the auditory nuclei, the number of impulses fired by cerebellar units changed either monotonically or non-monotonically with stimulus intensity. In the closed system study, the latency of an investigated unit to monaural stimulation was approximately the same as to binaural stimulation. Threshold curves of the same unit measured from each ear were also similar in shape and best frequency. Although all the units studied could be activated by stimuli delivered to either ear, the neural mechanism of binaural interaction is not a simple summation or occlusion. [Work supported by NSF BNS 80-07348 and USPH 1-K04-NS-00433-03 to P. Jen.] 


\author{
Session E. Engineering Acoustics I: Specialty Materials
}

\author{
Robert W. Timme, Chairman \\ Naval Research Laboratory, P.O. Box 8337, Orlando, Florida 32856
}

Chairman's Introduction-9:00

Invited Papers

9:05

E1. Specialty materials for underwater acoustics. Robert Y. Ting (Naval Research Laboratory, Underwater Sound Reference Detachment, P. O. Box 8337, Orlando, FL 32856)

In recent years, a majority of the failures of underwater transducers and sonar windows have been identified to be related to problems with the materials used in these systems. Most frequently, a design will successfully meet the initial performance requirement, but the device quickly fails after a short time of exposure to the service environment. The failure mechanisms often are attributable to the fact that the presently used materials are generally not optimized for long-term operation and always poorly characterized for their aging properties. In many cases, material components for a transducer may be selected from different sources, and this results in serious compatibility problems. Furthermore, because of the proprietary nature of many commercial materials, the user has very little control over the quality of the material, which often shows large batch-to-batch variations. This nonreproducibility has made it extremely difficult to properly repair or replace the failed acoustic devices. In order to increase the readiness of present sonars and to improve the lift-time performance requirement of future new transducers, it is necessary to understand materials' composition-property relationship, to develop quality control techniques and to design new materials for optimum applications. In this presentation, the major problem areas will be addressed, and progress made in broth the acoustically active and passive materials will be discussed.

\title{
9:35
}

E2. Twenty-year life hydrophones. A. C. Tims (Naval Research Laboratory, Underwater Sound Reference Detachment, P. O. Box 8337, Orlando, FL 32856)

A particular hydrophone design application has been chosen to demonstrate that high reliability can be achieved through the use of reliability modeling, an in-depth knowledge of transducer design, careful selection of materials, and good construction techniques. The reliability prediction for the design has been based upon random component failure with the assumption of a constant hazard rate. No consideration has been given to wear-out failure. The design features that increase reliability are presented; alternate design approaches, predictions of lifetime based upon reliability modeling, choices of materials, and successful operation of hydrophones to date are discussed. It is expected that this hydrophone will demonstrate that sonar equipment can be made with reliability equal to space-age equipment.

E3. Characterizing the impact of materials on transducer reliability. R. Lowell Smith (Texas Research Institute, 5902 W. Bee Cave Rd., Austin, TX 78746)

Achieving high reliability for an acoustic device depends ultimately on its design, materials of construction, fabrication processes, testing, correct installation, and the environment and conditions of use. This paper focuses on evaluating the impact of materials on transducer reliability. It is sometimes appropriate to characterize materials reliability using the familiar exponential reliability, or constant hazard rate model. Commonly in the marine environment, however, degradation processes operate which imply instead wearout reliability descriptions. Attention is focused on distinguishing, characterizing, and representing the reliability impact of these two situations. Failure definition, failure mode, and service limit concepts are introduced and their implications explored. The interpretation of chemical kinetics and other physical rate processes as the microscopic bases for macroscopic reliability descriptions is investigated. Under favorable circumstances this leads to the availability of time compression techniques referred to as accelerated testing, as a reliability evaluation tool. Suggestions are made concerning how reliability methods may be used to reduce the scope of the overall product or device improvement problem. Examples illustrating the use and implications of materials reliability methodology are presented. 


\section{Contributed Papers}

\section{0:20}

E4. Influence of chemical composition on dynamic properties of neoprenes for transducer applications. Rodger N. Capps and Corley M. Thompson (Naval Research Laboratory, Underwater Sound Reference Detachment, P.O. Box 8337, Orlando, FL 32856)

Elastomeric materials are widely used in the construction of wet-end sonar equipment. The viscoelastic nature of these materials causes them to possess and exhibit some extraordinary properties. Unless these properties are properly utilized, it can be disastrous to the performance of a transducer. Neoprenes are often used as components in transducers, due to the superior chemical and physical response properties of neoprene to many environments. However, the dynamic properties of neoprenes may often be inferior to those of othet types of elastomers. Since these properties are important for transducer applications, they should be optimized, with additives being used to provide desirable chemical and physical properties. In this paper, the effects of carbon black type and loading and variation of base polymer on a series of neoprenes have been investigated. The dynamic moduli and loss tangents have been characterized for a number of these elastomers. Based on the experimental results, the suitability of these materials for transducer applications and the optimization of dynamic properties of tranisducer elastomers will be discussed. [Work supported by NAVSEA 63R.]

\section{0:35}

E5. Some considerations on resistivity and permeability in the design of a reliable transducer elastomer. Corley M. Thompson (Naval Research Laboratory, Underwater Sound Reference Detachment, P.O. Box 8337, Orlando, FL 32856)

Neoprene elastomers are widely used as acoustic transducers but have rarely been optimized for their application. Electrical resistivity and water permeability are important properties in the design of a transducer elastomer. However, their dependency on the elastomer composite is generally unknown. A study was therefore designed to investigate the effects of different types and proportions of fillers on these pertinent physical properties. Three common neoprene types, each with a standard lead oxide cure system, were used. The dependency of resistivity on carbon black loading is found to be described by a sigmoidal curve for each carbonblack type. Ramifications of the frequently specified properties such as shore hardness and tensile strength on the resistivity will be discussed. Changes in electrical resistivity with seawater exposure will also be described. The dependency of the water permeability of the neoprene on some of the composition parameters has been determined. The effects of both of these variables on tranisducer lifetime and reliability will be discussed. [Work supported by NA VSEA 63X.]

\section{0:50}

E6. Elastomers in Mulloka sonar staves. D. Oldfield (Materials Research Laboratories, Department of Defence Support, Ascot Vale, Victoria, 3032, Australia)

This paper will present a case study of the use of elastomers in the underwater transducer array of the Australian designed Mulloka sonar system. The successful long-term operation of the sonar depends upon correct choice of materials to keep the interior of the array staves dry. A combination of factors led to severe corrosion inside the prototype staves which were subsequently refurbished with a butyl rubber coating over a cast polyurethane rubber membrane. Later laboratory studies showed that the sprayed butyl rubber coating was much more permeable to water vapour than a brushed coating. The rate of increase of relative humidity inside the array whilst in service with the Royal Australian Navy correlated well with the rate predicted from laboratory measurements. Experience with the prototype array clearly indicated the need for a much less permeable system for the production version. The design eventually adopted consisted of a pre-vulcanized bromobutyl rubber membrane bonded to the transducer heads and to the stave housing. The stave was then encapsulated in polyurethane to maintain the integrity of the rubber/ metal bonds. A limited amount of accelerated testing of actual staves gave moisture permeation results in good agreement with laboratory data. These results lend further support to the view that moisture ingress should not be a problem during the service lifetime of the transducer array.

\section{1:05}

E7. Water permeation through elastomer laminates. Patrick E. Cassidy (Texas Research Institute Inc., 5902 Bee Cave Road, Austin, TX 78746)

Neoprene and EPDM elastomers were used to measure the permeation rates of distilled water and salt water at $23^{\circ}, 40^{\circ}$ and $60^{\circ} \mathrm{C}$. For laminates, the rates were measured in both directions. The laminates showed a directional dependence of permeation rate and rates lower than the arithmetic mean of the two single layers. Effect of temperature on permeation rate had an Arrhenius type behavior and the activation energies fall within the range for those membranes that follow activated transport mechanism. Tentative mechanisms were proposed for the directional flow behavior in regard to concentration-dependent permeation.

\section{1:20}

E8. Fabrication of nonorthotropic axially stiffened polyurethane mandrels for use in fiber optic hydrophones. S. Africk, P. Jameson, and A. Ordubadi (Bolt Beranek and Newman Inc., 50 Moulton Street, Cambridge, MA 02138)

Ideally, extended hydrophones consisting of optical fibers wound on a compliant cylinder have high radial compliance to maximize sensitivity per unit length and high axial stiffness to minimize effects of longitudinal dynamics to assure uniform frequency response. Bendability is also desirable in certain applications. A set of nonorthotropic mandrels consisting of polyurethane cylinders with embedded axial steel wires have been fabricated. These include two levels of stiffening and polyurethanes of four degrees of hardness. Several aspects of the molding technique used, including process selection, mold design, air bubble requirements, and adhesion of the elements are described.

\section{1:35}

E9. Tonpilz head mass studies. George R. Douglas and Charles R. Wilson (Transducer and Acoustics Subsystems Design Engineering, Westinghouse Electric Corporation, Oceanic Division, Annapolis, MD 21404)

Alumina and composites of graphite/epoxy were used as head mass materials in two separate applications. In the first application alumina and composites of graphite/epoxy head masses were used to produce a low mechanical $Q$, high electroacoustic efficiency transducer. The initial results show that graphite/epoxy composites especially have great promise as head mass material because of its light weight and high tensile strength. In the second application an alumina head mass was used in a dual mode transducer. This configuration consists of an array of smaller tonpilz elements mounted in the head mass of larger tonpilz elements. This unique method required a very stiff secondary head mass to transfer acoustic energy (without storing strain energy) to the primary radiating surface of the smaller elements. Work in first application was sponsored by Westinghouse in-house R\&D Program of FY $81 \& 82$. [The work in second application was supported by the dual mode transducer program Navy contract No. N00140-80-R-6916.]

\section{1:50}

E10. Pressure release study of materials and configurations applicable to large sonar transducer. Charles $R$. Wilson (Transducer and Acoustics Subsystems Design Engineering, Westinghouse Electric Corporation, Oceanic Division, Annapolis, MD 21404)

Polyimide and polyester elastomer reinforced with $20 \%$ random glass were developed and tested as a pressure release material for sonar transducer applications. Tonpilz-type transducers using these materials as a 
pressure release were subjected to various high pressure conditions and intensive qualification tests including acoustic, extraneous noise, and shock. The results show that both polyimide and polyester elastomer reinforced with $20 \%$ random glass are excellent, cost efficient, pressure release configurations that can replace existing belleville springs and sonite pressure release systems now in use. [Work supported by Sonar Transducer Reliability Improvement Program FY 80 under Navy contract No. N00173-79-C-0390.]

\section{2:05}

E11. The speed of sound in epoxy resin blends and composites. Robert E. Montgomery, Fred J. Weber, David F. White (Texas Research Institute, 5902 Bee Caves Road, Austin, TX 78746), and C.
M. Thompson (Underwater Sound Reference Detachment, Naval Research Laboratory, Orlando, FL 32856)

Sound speeds in two types of diamine-cured epoxy resin blends were determined as functions of frequency, temperature, and composition, and the feasibility of impedance matching through the addition of microballoon fillers was also demonstrated. A filled resin system based on a diglycidyl ether of poly (propylene glycol) was shown to possess a mass density near $1.0 \mathrm{~g} / \mathrm{cm}^{3}$ and propagate sound at speeds near $150 \mathrm{~m} / \mathrm{s}$, while a resin system based on a diglycidyl ether of bisphenol A exhibited sound speeds and specific acoustic impedances about twice those of water. Although the incorporation of microballoons slightly increased sound speeds, the corresponding density reductions resulted in a net decrease of the specific acoustic impedance in all instances. [Work supported by NAVSEA 63R.]

TUESDAY MORNING, 9 NOVEMBER 1982

HILLSBOROUGH ROOM, 9:00 A.M. TO 12:05 P.M.

\title{
Session F. Musical Acoustics I and Psychological Acoustics I: Musical Perception and Cognition
}

\author{
Elizabeth A. Cohen, Chairman
}

Department of Physics, Stanford University, Stanford, California 94305

Chairman's Introduction-9:00

Invited Papers

9:05

F1. Music perception as detection of pitch-time constraints. Gerald J. Balzano (Department of Music, University of California, San Diego, La Jolla, CA 92093)

Music is viewed as constrained pitch-time structure. Perceiving music is taken fundamentally to be a process of detecting constraints in pitch-time information. These contraints emerge as invariants-under-transformation relative to certain mathematical groups. Groups for pitch and time structures are typically specified by an order relation and an equivalence relation. For pitch, the order relation is realized by pitch points that can be generated by a unit pitch interval ("semitone"), the equivalence relation by the octave. For time, the order relation is realized by time points that can be generated by a unit time interval, and the equivalence relation is created by the "measure" or "barline." By rendering pitch points separated by octaves as closely related, and similarly for time points at corresponding places in different measures, music reduces the number of its essentially different transformations drastically over pitch-time structures not so constrained, such as intonation contours in speech. In a number of experiments, listeners' ability to detect musical constraints is tested with "pseudomelody" stimuli-pitch-time structures consisting of approximately 80 note-events-exhibiting different kinds and degrees of constraint, and findings are reported. The present view of music perception as detection of existing structure is contrasted with current views of perception as construction or invention of structure, and a plea for a realist perspective in music research is expressed.

F2. Tw0-factor theory of auditory organization. Albert S. Bregman (Psychology Department, McGill University, 1205 Dr. Penfield Avenue, Montreal, Quebec, Canada H3A 1B1)

Evidence from several laboratories suggests a generalization that organizes several facts about auditory organization. It goes as follows: There are two "forces" of primary perceptual organization, a sequential one that organizes acoustic components sequentially into streams, and a simultaneous one that fuses components into pitch and timbre structures. Each of these forces draws its basic information from a separate physiological mechanism. The sequential force directly draws upon the place coding of the basilar membrane (BM). The simultaneous force is based upon timing information carried on neural outputs from the BM. Since the two forces compete, the factors that influence each can affect the other, but only indirectly. A parallel dichotomy is proposed for pitch: pitch height is controlled by BM place information while chroma is based on timing information. Hence pitch height should appear to influence sequential organization, and chromatic relations to affect fusion. Musical schemata can override these perceptual forces, but only at slow rates of presentation of sound. 
F3. Rhythmic and melodic strutures in perceptual space. Edward C. Carterette, Caroline B. Monahan, Eric Holman, Theodore S. Bell, and Robert A. Fiske (Department of Psychology and Brain Research Institute, University of California, Los Angeles, 405 Hilgard Avenue, Los Angeles, CA 90024)

By asking a person to say how much or how little alike two musical forms are, one can infer the dimensional properties of that person's musical space. We asked musically trained listeners to rate the similarity of pairs of brief melodies on a 9-point scale. Each of four different melodies and their inversions was played in each of four duration patterns (anapestic, dactylic, iambic, and trochaic) for a total of 1024 pairs of patterns. Multi-dimensional scaling and cluster analyses of the similarity matrices showed that at least five dimensions were necessary for giving a good account of the perceptual space of these musical structures. Surprisingly, the major dimensions were rhythmic, thus (using INDSCAL): (i) accent early or late, (ii) duple or triple meter, (iii) iambicdactylic versus anapestic-trochaic. Other dimensions were (iv) rising versus falling pitch and ( $v$ ) number of pitch-interval reversals. Cluster analysis suggests that some spaces have duple and triple meter as main branches, while others have accent first and accent last as main branches. Decision times (DT) were an inverted-U function of similarity $(S)$. The curve, when folded about $S=5$, was well fit by the power function $\mathrm{DT}(\mathrm{ms})=737 S^{027}$, with a coefficient of determination $R^{2}=0.98$.

\section{0:35}

F4. Contributions of sub-audio frequency modulation and spectral envelope constancy to spectral fusion in complex harmonic tones. Stephen McAdams (I.R.C.A.M., 31, rue Saint-Merri, F-75004 Paris, France)

Spectral fusion is a process by which elements of sound are grouped into unified auditory source percepts. The grouping processes use acoustic cues such as the presence of sub-audio frequency modulation, its coherence arnong spectral components and the constancy of the spectral envelope. Both periodic (vibrato) and random (jitter) modulation can contribute to fusion. The rms deviation of modulation must be greater than the modulation detection threshold found for these complex tones ( 16 harmonics of $220 \mathrm{~Hz}$ ). When the modulation imposed on the ensemble of spectral components is incoherent or does not maintain the harmonic relation (i.e., is not proportional to frequency for all harmonics), less fusion is perceived. There are indications that the detection of coherence of modulation occurs in the higher harmonic numbers. Those modulations which mitigate against fusion also contribute to an increase in spectral resolution. The spectral resolution indicated in these experiments appears to be greater than that for unmodulated signals classically reported. With more complex spectral envelopes, like vowels, a greater degree of fusion is reported when the spectral envelope is constant, i.e., a concurrent amplitude modulation accompanies frequency modulation and "traces out" the spectral envelope. This may indicate that grouping processes favor fixed-resonance structures. Musical examples illustrating the use of these principles in computer synthesis will be played.

\section{1:05}

F5. Musical key structure and its influence on perceived harmonic relations. Carol $L$. Krumhansl (Department of Psychology, Cornell University, Ithaca, NY 14853)

A structural description of musical key distance was derived from the tone-profile technique in which listeners rate how well each chromatic-scale tone follows a key-defining element (scale, tonic triad chord, or chord cadence). The rating profiles, reflecting the music-theoretic hierarchy of tonal stability, were shifted to all possible tonics, intercorrelated, and analyzed using multidimensional scaling. The four-dimensional solution placed the 24 major and minor keys on a torus simultaneously accommodating the circle of fifths and relative and parallel relations between major and minor keys. Two subsequent experiments investigated the effect of interkey distance on perceived harmonic relations between chords. The magnitude of three independent context effects (measured in both direct relatedness judgments and memory confusions) was found to depend systematically on the distance between the keys in which the chords function and the key of the broader context in which they are embedded. The results provide strong evidence that listeners interpret chords in their tonal functions and have internalized structure at the level of abstract tonal centers. [Work supported by NSF.]

\section{1:35}

F6. Chroma and interval in melody recognition: Effects of acquiring a tonal schema. W. Jay Dowling (Program in Psychology \& Human Development, University of Texas at Dallas, Richardson, TX 75080)

Pitch intervals of melodies in long-term memory are produced and recognized quite accurately by both musicians and nonmusicians. This could be either because memory represents intervals directly, or because pitches are represented as chromas in a tonal scale scherna (a do-re-mi-like representation). Changing chromas (but not intervals) of test melodies should disrupt recognition in the latter case, but not the former. Here I controlled chroma by introducing each melody with a chord sequence that ended with either a tonic (do-mi-sol) or dominant (sol-ti-re) chord. The melody was constructed around the root of that chord (do or sol). Recognition was tested with transpositions that either preserved or changed chord context. Listeners tried to distinguish between exact (interval-preserving) transpositions and near transpositions having one pitch altered. Inexperienced listeners succeeded whether chroma changed or not. Moderately experienced listeners succeeded only when chroma was preserved. The latter store melodies in terms of chromas and have difficulty recognizing them when chromas are changed. This result converges with others relating schema use and musical experience. 


\title{
Session G. Physical Acoustics I: Timely Topics
}

\author{
Steven L. Garrett, Chairman \\ Department of Physics and Chemistry, Naval Postgraduate School, Monterey, Califormia 93940
}

Chairman's Introduction-9:00

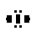

Invited Papers

iị.

9:05

G1. Intrinsically irreversible acoustic heat engine. John C. Wheatley (Group P-10, MS-M764, Los Alamos National Laboratory, Los Alamos, NM 87545)

Thermodynamic effects will be described associated with structures placed within a gas contained in an apoustically resonant tube. For suitable geometries and frequencies substantial temperature differences, perhaps 50 times the adiabatic temperature changes in the gas, can be produced. The experiments can be understood in a context of certain principles, including the principle of broken thermodynamic symmetry, which describe intrinsically irreversible reciprocating heat engines. [Work supported by LANL and by the D.O.E.]

$$
\text { 1: }
$$

9:35

G2. Acoustic streaming induced by the "vortex whistle" is the cause of the Ranque-Hilsch effect. M. Kurosaka, J. R. Goodman, and J. Q. Chu (Department of Aerospace and Mechanical Engineering, University of Tennessee Space Institute, Tullahoma, TN G7 388)

In 1933, Ranque discovered a striking phenomenon: When pressurized air is injected tangentially into a pipe and imparted a swirling motion, its total temperature separates into a colder one at the centerline and a warmer one at the periphery. The separationtakes place spontaneously, without the aid of any external mechanical work. The effect became populariztd after the Second World War by the work of Hilsch; the socalled Ranque-Hilsch tube or the vortex tube is now commercially available. Even for inlet antit only a few atmospheric pressure and at room temperature, the total temperature at the tube center can easily become freezingly cold; the total temperature at the periphery exceeds its upstream value correspondingly. Although there exists a general consensus that some unsteadiness is needed to account for this phenomenon, the crucial 3*:- issue as to the precise form of unsteadiness or its explicit mechanism has never been resolved. Now, it is a known fact that a pure tone of high intensity or vortex whistle often emanates from the vortex flow, the Ranque-Hilsch tube included; its frequency increases proportionally to the swirl. We submit here that acoustic streaming induced by the vortex whistle is the mechanism of the Ranque-Hilsch effect. We demonstrate this, based on our recently published work [Kurosaka et al., J. Fluid Mech. (in print)] and on additional results obtained subsequently. [Work supported by the Air Force Office of Scientific Research.]

\section{0:05}

G3. Spherical acoustic resonators for thermophysical property and standards measurements. Michael R. Moldover and James B. Mehl') (Thermophysics Division, National Bureau of Standards, Washington, DC 20234)

t. We are studying the feasibility of using the resonances of $a$ low density gas enclosed by a thick spherical shell for measuring various thermophysical properties, including the thermodynamic temperature, and the universal gas constant $R$. The radially symmetric resonances have high $Q$ 's $(2000-10000)$. Thus very simple instrumentation can be used to measure the speed of sound in a gas with an accuracy of $0.02 \%$. We have used more elaborate instrumentation to measure resonance frequencies, $f_{x}$, and resonance halfwidths, $g_{x}$, with a precision on the order of $10^{-7} f_{x}$. The ratios of $f_{x}$ for various resonances, extrapolated to zero pressure, are within $\pm 3 \times 10^{-6}$ of the ratios predicted 1 by our theoretical model. For the same resonances, the measured values of $g_{x}$ exceed the theoretical values by less than $5 \times 10^{-6} f_{x}$. These results suggest that an acoustic measurement of the thermodynamic temperature or the gas constant could be accurate to $0.001 \%$. The simple geometry of this oscillating system makes it easy to incorporate many phenomena in a theoretical model. We have included the following boundary effects: (1) the thermal boundary layer; (2) shell elasticity; (3) transducer coupling holes and a fill hole; (4) adsorption and desorption; (5) geometric imperfections, and (6) the viscous boundary layer (for nonradially symmetric modes). "Also: Physics Department, University of Delaware, Newark, DE 19711.

$+1+$ 
G4. An acoustic technique for measurement of electric fields inside dielectrics. A. Migliori (Los Alamos National Laboratory, Los Alamos, NM 87545)

If a dielectric containing an electric field is compressed locally by a narrow acoustic pulse, the potential difference changes between the boundaries of the dielectric along the direction of travel of the pulse. This change in potential is dependent on the compressibility of the dielectric, on the pressure dependence of dielectric constant, and on the local macroscopic electric field in the region being compressed [A. Migliori and T. Hofler, Rev. Sci. Instrum. 53, 662 (1982)]. Because the pulse travels at the speed of sound, the potential difference is time dependent and can be used to map the internal electric field over the path of the pulse. We describe the theory of this measurement technique and present data taken with laser-generated acoustic pulses. The data demonstrate a sensitivity of $5 \times 10^{4} \mathrm{~V} / \mathrm{M}$ and a resolution of $50 \mu \mathrm{M}$ with an absolute accuracy of $\pm 5 \%$. [Work supported by U. S. Department of Energy.]

\section{1:05}

G5. Electron-acoustic microscopy. G. S. Cargill III (IBM Thomas J. Watson Research Center, P. O. Box 218, Yorktown Heights, NY 10598)

Electron-acoustic microscopy is a technique which combines electron beams and ultrasonics to examine spatial variations in thermal and elastic properties of solids on a microscopic scale [G. S. Cargill III, Nature 286, 691 (1980); Phys. Today 34, 27 (Oct. 1981); E. Brandis and A. Rosencwaig, Appl. Phys. Lett. 37, 98 (1980)]. A focused electron beam is chopped at $\mathrm{kHz}$ or $\mathrm{MHz}$ rates to generate ultrasonic waves by thermoelastic expansion at the front surface of a specimen, and these are detected by a piezoelectric transducer bonded to the specimen. The transducer output is used to form a scanned, magnified image containing near-surface and subsurface information which is not accessible in conventional optical or electron microscopy. This mode of ultrasonic imaging in scanning electron microscopy may be called electron-acoustic microscopy in analogy with photoacoustic microscopy [G. Busse and A. Rosencwaig, Appl. Phys. Lett. 36, 815 (1980)] in which a laser is similarly used to generate acoustic or ultrasonic waves. However, the electron beam can be focused to a much smaller spot than is possible with visible light, yielding improved spatial resolution. [This work was begun at the University of Cambridge, with support from the Science Research Council and Churchill College.]

\section{Contributed Papers}

\section{1:35}

G6. Ultra-deep subharmonics in high amplitude shallow water waves. Robert Keolian and Isadore Rudnick (Department of Physics, University of California, Los Angeles, CA 90024)

When a horizontal annular channel containing water is parametrically excited by oscillating it vertically with moderately high amplitude at a frequency $f \simeq 10 \mathrm{~Hz}$, then under appropriate conditions nearly periodic subharmonics of frequency $f / 2000$ are generated. [Earlier work on this problem can be found in Phys. Rev. Lett. 47, 1133 (1981)]. This oscillation is robust and has been observed continuously for a period of $30 \mathrm{~h}$ (a million cycles of the driving frequency). The basic nature of the oscillation is that of a modulated $f / 2$ mode. In another experiment when waves in a linear trough are driven by a horizontally oscillating paddle, a modulated $f / 3$ mode is observed with a basic subharmonic of approximately $f / 100$. The pattern of the motion as shown by a strip chart recorder has the appearance of an intricate braid. Other ultradeep subharmonics have also been observed. The origin of these subharmonics will be discussed and a videotape will be presented. [Work supported by ONR.]

\section{1:50}

G7. Nonlinear thermoacoustics and electrostriction explanations of acoustic signatures in $4^{\circ} \mathrm{C}$ water caused by laser pulses and proton beams. Allan D. Pierce (Georgia Institute of Technology, Atlanta, GA 30332)

Experiments in water with proton beams [Hunter et al., J. Acoust. Soc. Am. 69, 1557-1562(1981)] and with laser pulses [Hunter, Jones, and Malbrough, J. Acoust. Soc. Am. 1563-1567 (1981)] give pressure signatures superimposed of a bipolar signal (reversing polarity at $4{ }^{\circ} \mathrm{C}$ ) and an anomalous tripolar signal that at onset is (1) negative (proton beams) or (2) positive (laser pulses). The explanation for (1) is that charge added by a proton beam causes a surrounding short-lived electric field that, via electrostriction, initially accelerates fluid inwards and temporarily lowers pressure in an annular region and raises it in the core. Such causes a minus-plus-minus-plus shaped signature varying inversely with electrical conductivity. The explanation for (2) ensues from a formulation that uses the thermal expansion coefficient's temperature dependence. Such yields an additional source term (nonzero at $4^{\circ} \mathrm{C}$ and proportional to negative of second time derivative of square of heat added per unit volume) to inhomogeneous wave equation, resulting in initially positive signature. Pulse durations and charge relaxation times are assumed short compared to acoustic transit time across diameter, so all signature time scales are of order of latter. Rough numerical estimates are not inconsistent with reported crossovers in initial polarity at $6^{\circ}$ and $3{ }^{\circ} \mathrm{C}$, respectively. The small second positive peak in the tripolar signal and the precursors in the laser beam experiments are still not understood. [Work supported by ONR.] 


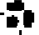

\section{Session H. Shock and Vibration I: Fatigue in Structures}

\author{
Sabih I. Hayek, Chairman
}

Applied Research Laboratory, Pennsylvania State University, P. O. Box 30, State College, Pennsylvania 16801

\author{
Chairman's Introduction-9:00
}

\section{Invited Papers}

\section{9:05}

H1. Nondestructive detection of fatigue damage. Robert E. Green, Jr. (Materials Science and Engineering Department, The Johns Hopkins University, Baltimore, MD 21218)

This paper will discuss the various nondestructive techniques which have been used or which are potentially useful for detection of fatigue damage. The ideal nondestructive testing technique would permit very early detection of fatigue damage so that proper assessment of the severity and rate of severity increase of the structural damage leading to failure can be made. Thus, the most sensitive systems would be capable of detecting motion, pileup, and breakaway of dislocations; the next most sensitive systems would be capable of detecting microcracks; the least sensitive systems would only be capable of detecting macrocracks. It is practically expedient to have nondestructive techniques which can successfully detect fatigue damage in each of these regimes since some components can tolerate larger amounts of fatigue damage or larger crack sizes than others without serious concern for the structural integrity of the component. It will be shown that the best nondestructive technique for detecting and sizing of macrocracks is ultrasonics; the best nondestructive techniques for detection of microcrack formation and possible pre-microcrack fatigue damage are ultrasonic attenuation and acoustic emission; a large htmber of nondestructive techniques for residual stress (strain) measirements are candidates for extremely early fatigue damage detection, with current interest primarily directed at ultrasonic wave velocity measurements.

H2. Ultrasonic characterization of fatigue in advanced composite materials. John H. Cantrell, Jr. (NASALangley Research Center, Mail Stop 499, Hampton, VA 23665)

In contrast,to fatigue of metals which tends to originate from crack initiation at identifiable defect sites, fatigue of fibef-Feinforced composite materials has not been identified with a single dominant failure process. Rather, fatigue of such materials is manifested by several damage mechanisms including through-the-ply cracks, fiber disbonds, and delaminations. The presence of multiple damage mechanisms gresents special problems both in understanding the fundamental nature of failure in fiber/matrix composites as; ${ }^{+}$ell as in their nondestructive evaluation. Ultrasonic methods for characterizing fatigue damage in fiber/matrix composites will be examined with special emphgsis on quantitative measurements of attenuation, velocity, and the values of the linear and quadratic attenuation coefficients measured as a function of frequency. The effect of ultrasonic phase cancellation etrors due to material anisotropy and inhomogeneity as well as the influence of pulse shape artifacts on quantitative measurements are discussed.

\section{0:05}

H3. Ultrasonic studies of mechanical properties of materials. A. V. Granato (Department of Physics and Materials Research Laboratory, University of Illinois at Urbana-Champaign, Urbana, IL 61801)

In recent studies, the close relationship between ultrasonic attenuation and the plastic flow of solids has become more explicit. Both depend sensitively upon the details of dislocation-defect interactions. The interactions can be separated into those producing pinning and those producing viscous drag. Deformation behavior: can be classified according to the strength of the drag. For small drag, as in superconductors, inertial effects:become important. For intermediate drag, traditional theories resting on rate theory treatments become applicable. For large drag, viscoelastic behavior is obtained. Measurements are examined for information concerning the basic nature of different sources of short- and long-range pinning and of drag.

H4. Interaction fultrasonic waves with cracks. Laszlo Adler artments of Welding Engineering and Engineering Mechánics, Ohio State University, Columbus, OH 43210)

An overview is presented on recent studies of ultrasonic waves interacting with artificial and real cracks. Various experimental methods will be described to obtain quantitative information concerning both geometrical [such as size, orientation] parameters as well as physical and surface parameters [such as stress intensity, 
surface roughness, distribution functicn, closure] of cracks. Various analytical approaches to support experimental results and carry out inyersions will also be emphasized. [Work supported by DARPA/AF through Ames Laboratory.]

\section{1:05}

H5. Application of $x$-ray diffraction to monitor fatigue deformation in high strength alloys. Robert N. Pangborn (Department of Engineering Science and Mechanics, The Pennsylvania State University, University Park, PA 16802)

A number of $\mathrm{x}$-ray diffraction techniques have been applied to measure the fatigue deformation introduced during axial and flexural loading in high strength aluminum and nickel base alloys. X-ray double-crystal diffractometry and $x$-ray line-broadening analyses were conducted to evaluate the localized and average microplasticity, respectively, generated prior to crack initiation. The modifications in diffraction peak profiles recorded using these techniques were interpreted in terms of changes in lattice distortion and dislocation density and correlated to the surface topographic features developed during fatigue cycling. These methods were also utilized to examine the fatigue deformations accrued in the subsurface and bulk of the samples. Both stepwise removal of surface layers and nondestructive evaluation using $x$ rays of different penetration capability were employed to obtain the depth profiles. X-ray stress measurements were made to disclose the modifications in residual stress occurring during fatigue, their relationship to the dislocation distribution, and their role in crack nucleation. The combination of $x$-ray methods provided information useful both to identification of the failure mechanism and to monitoring cumulative deformation preceding ultimate failure, even under spectrum amplitude testing conditions.

TUESDAY MORNING, 9 NOVEMBER 1982

ORANGE ROOM, 9:00 A.M. TO 12:07 P.M.

\section{Session I. Speech Communication I: Speech Perception}

Carl E. Williams, Chairman

Naval Aerospace Medical Research Laboratory, Pensacola, Florida 32508

Chairman's Introduction-9:00

\section{Contributed Papers}

9:05

I1. Perceptual scale for voice onset time (VOT) in synthetic / bae/ /pae/ syllables. B. Espinoza-Varas (Psychology Department, McGill University, Montreal, Canada, H3A 1B1)

The scale used by the perceptual system to represent variations in VOT appears to be different from the physical VOT scale. The perceptual scale, ranging from voiced to voiceless, has zero point at the phonetic boundary (the zero voiced-voiceless point). The degree of voicedness (voicelessness) increases with the distance between the VOT value and the boundary. Further, the perceptual effect of a difference in VOT near the boundary is greater than at points remote from the boundary. A rescaling of the VOT continuum that is more consistent with the properties of the perceptual scale is given by the function $D x_{i}=k\left|x_{i}-x_{b}\right|^{A}, 0<\beta<1$, where $x_{i}$ is a VOT stimulus value, $x_{B}$ the boundary VOT, $k$ a constant, and $D x_{i}$ the perceptual magnitude of $x_{i}$ along the voiced (voiceless) scale. The perceptual distance, $\Delta D$, between two stimuli $x_{i}, x_{i+1}$, is given by the difference between $D x_{i}$ and $D x_{i+1}$; that is, $\Delta D=k|| x_{i+1}-\left.x_{B}\right|^{\theta}$ $-\left|x_{i}-x_{B}\right|^{\beta} \mid$. The power transformation at the perceptual level is suggested by the following: (1) the relation between cumulative disciminability and cumulative distance from the boundary, in $\log$-log coordinates, is well described by straight lines with positive slopes smaller than one. This is observed in /bae/ / pae/ discrimination data obtained in the present and in previous studies [Elman, J. Acoust. Soc. Am. 65, 190-207 (1979)]; (2) for a constant msec difference between $x_{i}$ and $x_{i+1}$ the function $\Delta D$ reaches a maximum when the pair straddles the boundary, and decreases monotonically as the distance between the stimuli and the boundary in- creases; (3) changes in discriminability associated with changes in the size of the VOT steps of the /bae//pae/ correlate strongly with changes in $\Delta D$.

I2. A perceptual study of the voiced-voiceless contrast in syllable-final stops. Dennis Ingrisano, James Hillenbrand, Bruce L. Smith, and James E. Flege (Speech Research Laboratories, Department of Communicative Disorders, Northwestern University, Evanston, IL 60201)

Computer editing techniques were used to remove voicing in 10-ms steps from syllable-final closure intervals of natural tokens of [peb,ped,peg, pag, pig, pug]. Release bursts were retained and a pitchsynchronous editing routine was used to equalize vowel durations at about $110 \mathrm{~ms}$. Identification results from 23 listeners showed that, in general, relatively large amounts of voicing had to be removed from the closure intervals before perception changed from voiced to voiceless. For some of the continua (e.g., /peb-p\&p/), voiceless responses began to predominate when more than $95 \%$ of the closure interval was rendered voiceless. For other continua (e.g., /pag-pak/), the change from voiced to voiceless responses did not occur until voicing during the closure interval and a portion of the VC transition were removed. We found no place effect for the syllable-final stop; a significant, but relatively small effect for the vowel context was observed. In a second experiment, 11 listeners identified stimuli from which the release bursts had been excised. No significant 
differences were found between identification boundaries with or without bursts. Findings are discussed in relation to the nature of voicing cues in syllable-final stops. [Work supported by NIH: T32 NS 07100-04 and NIH Biomedical Sciences Support Grant: 5 S05 RR07028.]

\section{$9: 33$}

13. Perceptual and acoustic invariance for place of articulation in diffuse stop consonants. Letitia Gewirth, Sheila E. Blumstein, Aditi Lahiri, and Kathleen Kurowski (Department of Linguistics, Brown University, Providence, RI 02912)

A recent investigation of acoustic invariance for place of articulation in diffuse stop consonants [Lahiri and Blumstein, J. Acoust. Soc. Am. Suppl. 1 70, S39 (1981)] has shown that current theories of acoustic invariance cannot distinguish labial from dental stops. Analysis of natural speech utterances suggested that there was acoustic invariance for labial and dental consonants, but based on the relative changes in the distribution of energy at high and low frequencies from the burst release to the onset of voicing. In this study, we attempted to determine whether the relative changes in the spectral characteristics elaborated by Lahiri and Blumstein would play a role in the perception of place of articulation in diffuse stop consonants. Prototype CV syllables ([b d] in the context of [i e a $o$ u]) were generated based on natural speech tokens. The gross shape of the spectrum was manipulated first at the burst onset, then at the onset of voicing, such that the stimulus configuration had the spectral properties for labial or dental consonants while the formant frequencies and transition values were appropriate to the contrasting place of articulation. Results showed perceptual shifts for both labial and dental consonants in the context of $[i]$ and [e], but not for the back vowels. Changing the prototype stimuli for the latter by using a mid-value $F 2$ in the test stimuli did have some perceptual effects on place of articulation. [Supported in part by an NIH grant.]

\section{9:47}

I4. When is a [p] a [t], and when is it not. Richard E. Pastore, Rosemary Szczesiul, Lawrence D. Rosenblum, and Mark A. Schmuckler (Department of Psychology, SUNY-Binghamton, Binghamton, NY 13901)

Under certain dichotic listening conditions the initial stop $[p]$ in a naturally spoken word may be perceived as [ $t$ ]. The words were monaural with Gaussian noise in the contralateral earphone. Both naive subjects and knowledgeable subjects provided a psychophysical mapping of the conditions necessary for the transformation. The transformation occurs only in the context of certain vowels (occurring consistently in the words "pam," "pat," and "par"). The intensity, relative temporal onset, and to a lesser extent, the duration of the contralateral noise, are important parameters. The phenomenon clearly is dependent upon dichotic fusion. When the stimuli were mixed and presented monaurally, subjects either correctly reported the nature of the mixed stimuli (for low noise intensities) or reported hearing the noise plus the word without an initial consonant (for higher noise intensities acting as a masking stimulus). There are considerable individual differences in the tendency to perceive the transformation. To date we have not found any conditions where the dichotic noise will alter the perception of an initial [ $t$ ] or [b] other than in a strictly maskingtype fashion. [Research supported by NSF grant BNS-8003704.]

\section{0:01}

I5. Limits on the power of silence as a stop manner cue. Bruno $H$. Repp (Haskins Laboratories, 270 Crown Street, New Haven, CT 06510)

Previous research has shown that a bounded interval of silence is an important perceptual cue to stop manner, and that it may be both necessary and sufficient. However, most earlier stimuli did not include stop release bursts. When a release burst is present, a preceding interval of silence is frequently not necessary for perception of a stop, particularly when place of articulation is alveolar or velar. New experiments varying burst duration and amplitude reveal listeners' exquisite sensitivity to burst information, suggesting that the release burst, and not silence, is really the primary cue to stop manner. As to the sufficiency of the silence cue, it is well-known that a reasonable stop percept can be created by splicing sllence into a previously stopless utterance (e.g., $/ \mathrm{slit} / \rightarrow /$ split $/$ ). However, new experiments show that under certain conditions listeners readily perceive these modified utterances as two stopless pieces separated by silence (e.g., /s-lit/). Stop perception, in these cases, seems to depend on an integrative perceptual strategy that, however, can be disengaged without leaving the speech mode. Thus, silence alone is not always sufficient for stop perception. [Work supported by NICHD.]

\section{0:15}

I6. Nonmonotonic discrimination functions for temporal gaps in noisebuzz sequences. II: Filtering affects the discrimination peaks. Pierre L. Divenyi (Speech and Hearing Research Facility, V. A. Medical Center, Martinez, CA 94553 and Department of Otolaryngology, University of California, San Francisco, CA 94143)

It has been reported [P. L. Divenyi and W. F. Danner, J. Acoust. Soc. Am. Suppl. 1 55, S31 (1975) and J. D. Miller et al., J. A coust. Soc. Am. 60 $410-417(1976)]$ that discrimination of the gap bounded by a brief, filtered noise burst and a longer, /a/-like buzz is a nonmonotonic function of the gap duration, in a way similar to the well-known nonmonotonicity of the VOT discrimination functions [A. S. Abramson and L. Lisker, Proc. 6th Int. Congr. Phonet. Sci., 569-573(1970)]. Earlier, we reported that discriminability of the gap in such sequences is affected by passing the stimulus through 1/3-octave filters and that the discrimination thresholds are correlated with the difference between noise and buzz intensity within the particular bands-i.e., with one of the main attributes of temporal masking. The present experiments extend the previous ones, in that the continuum of "buzz-onset-times" was explored in finer steps $(10,15,20,25,30$, $35,40,55$, and $70 \mathrm{~ms}$ from noise onset to buzz onset). In addition to determining gap discrimination thresholds for the original, unfiltered stimulus, thresholds were also obtained at three filter settings $(780,1230$ and $2550 \mathrm{~Hz}$ center frequencies). Three trained listeners participated. While the smallest thresholds at the 780 and $1230-\mathrm{Hz}$ filter settings occurred at the same gap duration as for the unfiltered stimulus $(15 \mathrm{~ms})$, the threshold minimum at the $2550-\mathrm{Hz}$ filter setting occurred at longer gap durations (20 and $25 \mathrm{~ms}$ ). Implications of these results for variations in voicing category boundaries as well as for the possible role of listening bands in the perception of speech will be discussed. [Work supported by NIH Grant NS16019 and by the Veterans Administration.]

\section{0:29}

17. Laterality differences in speaker and consonant identification in dichotic listening. V. C. Tartter (Psychology Department, Rutgers University, Camden, NJ 08102 and Bell Laboratories, Murray Hill, NJ 07974)

Two dichotic listening experiments were conducted using the syllables [ba], [da], [pa], and [ta] each spoken by two male and two female speakers. Twenty right-handed subjects participated in each experiment. Each experiment consisted of two parts with order counterbalanced across subjects: in one, subjects identified the two consonants; in the other, after a brief training session to familiarize them with the speakers, they identified the two speakers. The experiments differed in whether the nontarget dimension varied. In experiment 1 if subjects were identifying speaker, the same consonant was presented to both ears on a given trial, whereas in experiment 2 both speaker and consonant varied. Both experiments resulted in significant right-ear advantages for consonant identification, typical feature-sharing advantages and blend errors for consonants, significantly fewer errors in speaker identification than consonant identification, and greater confusion between same-sexed voices than different-sex voices. Experiment 1 showed no significant ear advantage for speaker identification while experiment 2 revealed a marginal right-ear advantage. The difference is attributed to greater involvement of the left hemisphere incurred by the extra phonetic variation.

\section{0:43}

I8. Perceived manner and the intervocalic place cues of $/ \mathrm{s} /$. George P. McCasland (4045 Baltimore Avenue, C3, Philadelphia, PA 19104)

A set of 12 pairs of CVCV /sısi,sisa/ tokens spoken by one male were 
subjected to PCM editing to remove the friction noise of the intervocalic /s/. In a perceptual identification test subjects judged the consonants represented by the intervocalic silent intervals as one of $/ p, t, s, f, \theta /$. In a second test subjects were forced to choose only fricatives $/ f, \theta /$. For the /sisi/ series of tokens with the $/-\mathrm{i} /$ final vowel, when the manner judgment was a stop, it was predominătely $/ \mathrm{p} /$ indicating a labial place cue. When the manner judgment was a fricative, it was predominately $/ \theta /$ indicating a dental-alveolar cue. Perceived place depended on perceived manner. For the /sisa/ series, a manner-place effect was not apparent; nearly all judgments were $/ t /$ or $/ \theta /$. Clearly, any association between place and manner judgments may be specific to the vocalic context. This investigation extends the work previously reported by the writer [J. Acoust. Soc. Am. Suppl. 171, S105, 1982] and the work of Cardin, Levitt, Jusczyk and Walley [Percept. \& Psychophys. 29, 26-36 (1981)]. [Haskins Laboratories provided valuable assistance in preparing speech samples on NICHD Contract No. NIH-71-2420.]

\section{0:57}

19. Signaling of syntactic structure in whispered speech. Ilse Lehiste (Department of Linguistics, Ohio State University, Columbus, $\mathrm{OH} 43210$ )

Several recent studies have shown that the internal structuring of a sentence can be signaled by manipulation of the time domain [for a review, cf. R. Scott, J. Acoust. Soc. Am. 71, 996-1007 (1982)]. Other studies have associated the signaling of syntactic boundaries with specific pitch movements, such as a fall-rise in the intonation contour [cf. W. E. Cooper and J. M. Sorensen, J. A coust. Soc. Am. 62, 683-692 (1977)]. An experiment was designed to explore whether there is any difference in the perception of syntactically ambiguous sentences produced in two modes: with normal phonation and in whisper. Two speakers of American English recorded phonated and whispered versions of a list of 20 sentences containing instances of four syntactically ambiguous sentences. Twenty-five listeners identified the meanings intended by the speakers. The correct scores, averaged for the two speakers, were $97.1 \%$ for phonated speech and 97.6\% for whispered speech. Analysis of the sentences showed that while whispered sentences were somewhat longer than phonated sentences, the speakers had made no special effort to compensate for the absence of $F O$ cues by exaggerating durational cues.

\section{1:11}

I10. Accuracy and criterion in the perception of speech by young and old listeners. Jerry L. Yanz and Shirley M. Anderson (Department of Communication Disorders, University of Minnesota, 115 Shevlin Hall, Minneapolis, MN 55455)

Several authors have used signal detection theory (SDT) to demonstrate that older listeners use stricter criteria than younger listeners in detecting tones in noise [e.g., J. N. Rees and J. Botwinick, J. Gerontol. 26, 133-136 (1971); M. Potash and B. Jones, J. Gerontol. 32, 436-440 (1977)]. Our aim was to determine if such criterial differences also exist in the perception of speech. The identification of monosyllables (CID W-22 lists) presented in a broadband noise background ( $\mathrm{S} / \mathrm{N}=0$ or $5 \mathrm{~dB}$ ) was evaluated in young and old listeners. Immediately after identification of a test item, listeners rated the accuracy of their own identification on a six-point scale [see I. Pollack and L. R. Decker, J. Acoust. Soc. Am. 30, 286-292 (1958)]. Performance accuracy and decision criteria were separated according to a SDT analysis. Data showed no difference in criterion between young and old groups and a difference in accuracy only for the more favorable signal-to-noise ratio. Two performance measures will be discussed-percent correct, the conventional measure in speech audiometry, and $\boldsymbol{P}(\boldsymbol{A})$, the nonparametric measure of performance accuracy in SDT with regard to the quantification of perceptual accuracy and communication effectiveness.

\section{$11: 25$}

I11. Multidimensional scaling analysis of symmetric and nonsymmetric consonant confusion matrices: A comparison of different proximity measures. Moshe Yuchtman and Robert C. Bilger (Department of Speech and Hearing Science, University of Illinois, Champaign, IL 61820)

In a recent paper [Yuchtman and Bilger, J. Acoust. Soc. Am. Suppl. I 71, S75 (1982)] we reported that two proximity measures, namely, Ln S
[Soli and Arabie, J. Acoust. Soc. Am. 66, 46-59 (1979)] and the index of sensitivity, d', yielded similar multidimensional solutions in SINDSCL analysis of consonant confusion matrices. We have now studied these measures in a comparative analysis of symmetric and nonsymmetric consonant confusion matrices, expecting differences between $\mathrm{Ln} S$ and $d^{\prime}$, since the first measure, unlike the second assumes matrix symmetry. 48 consonant confusion matrices of 24 subjects were analyzed. For each subject stimuli were presented with presence of noise or in the quiet in procedure similar to the one described by Wang and Bilger [J. Acoust. Soc. Am. 69, 832-845 (1973)]. An index of symmetry was obtained for each matrix by using the procedure suggested by Hubert and Baker [Qual. Quant. 13, 77-84(1979)]. The 12 matrices with the highest or lowest symmetry scores were subjected to further analysis. For each of these matrices we calculated three proximity measures: $S$, $L n S$, and $d^{\prime}$. They were then analyzed using the SINDSCL program. The results indicate that: (1) for all of these measures, a given $n$-dimensional configuration accounted for larger proportion of the variance in the high-symmetry than in the low symmetry matrices; and (2) for both types of matrices, $d^{\prime}$ and $\mathrm{Ln} S$ accounted for roughly the same proportion of variance which was higher than that yieided for $\mathrm{S}$. We are now analyzing the specific solutions obtained for each of these measures. [This work was supported by NINCDS.]

\section{1:39}

112. Absolute judgments of speech rate as a function of vocal frequency and intensity. Stanley Feldstein (Department of Psychology, University of Maryland Baltimore County, Catonsville, MD 21228), Ronald N. Bond (Department of Psychology, New York University, New York, NY 10003), Sallie Simpson, and Elizabeth Brady (Department of Psychology, University of Maryland Baltimore County, Cantonville, MD 21228)

Previous studies have found that vocal frequency and intensity both influence the perception of speech rate when a standard, or "anchoring," stimulus preceded each experimental stimulus. The two studies reported here replicated the previous studies but omitted the anchoring stimulus. In one study, 30 judges rated nine "content-masked" speech stimuli generated from a 20 -second female speech samiple by factorially combining three levels each of manipulated frequency and intensity. In the other study, 29 judges rated 18 content-standard stimuli generated from two 20 $s$ speech samples, one from a woman and one from a man. The actual rate of each stimulus was $150 \mathrm{wpm}$. With the masked stimuli, frequency and intensity separately influenced the perception of speech rate. With content-standard stimuli, frequency and intensity jointly affected speech-rate perception. Moreover, the joint influence was affected by the gender of the speaker; stimuli from the male speaker were judged to be faster. The studies suggest that an anchoring stimulus is not needed to demonstrate the influence of frequency and intensity on speech-rate perception.

\section{1:53}

113. The influence of vocal frequency and intensity on the perception of speech rate: Natural speech samples as stimuli. Ronald N. Bond (Department of Psychology, New York University, 6 Washington Place, New York, NY 10003), Stanley Feldstein, Elizabeth Brady, and Sallie Simpson (Department of Psychology, University of Maryland Baltimore County, Catonsville, MD 21228)

Previous studies by the authors found, using masked speech samples, that the perception of speech rate was influenced by both vocal frequency and intensity. The study reported here replicated the previous one but used unmasked, or natural, speech stimuli and examined the effects of speaker gender. Using one speech sample each from a male and female speaker, three levels of frequency and intensity were factorially combined to produce nine stimuli for each speaker. The actual speech rate of each stimulus was $150 \mathrm{wpm}$. The 18 stimuli were randomly ordered with each preceded by a standard stimulus. Twenty-nine judges rated each stimulus, relative to the standard, on four 7-point, unipolar scales: speech rate, pitch, loudness, and duration. The results indicate that the perceptions of speech rate were positively related to frequency but not intensity. Judgments of pitch and loudness were positively related to frequency and intensity, respectively, and judgments of duration were inversely related to frequency. Finally, the influence of frequency on perceived speech rate differed for the male and female speaker. 


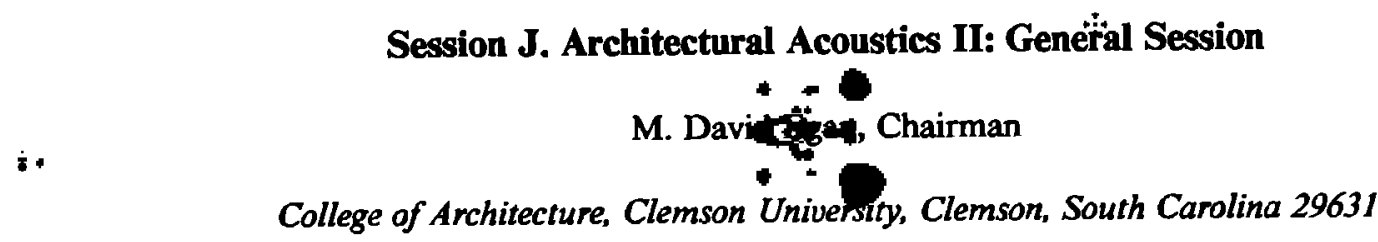

Chairman's Introduction-10:20

Invited Paper

if.

10:25

J1. Coñcert hall acoustics and number theory? Acoustic precision measurements with husic?? Manfred R. Schroeder (Drittes Physikalisches Institut. University of Göttingen, F. R. d. Gffithany and Bell Laboratories, Murray Hill, NJ 07974) :

Work at the Drittes Physikalisches Institut, University of Göttingen, on acoustic quality of concert halls is reviewed. Methods for better sound diffusion by reflection phase-gratings, based on three different principles of number theory, are described. The concept of the "modulation transfer function" (MTF) is exploited to use the music itself, as played during regular concerts in the presence of a full audience, to measure such important room acoustical parameters as reverberation time and early-to-late energy ratio.

+1.

\section{: Contributed Papers}

11:25

$+\bar{x}$

J2. Listening systems-Application analysis. Anna $K$. Nábžlek, Tomasz Lętowski, and Igor V. Nábělek (Depart'ment of Audiology and Speech Pathology, University of Tennessoe, Knoxville, TN 37996-0740)

For many years, listening systent have been used for the hearingimpaireds in schools for the deaf and the general public for simultaneous transmi cion in several languages. Recently, listening systems started to be instafied for pleasure listening in theaters, churches and concert halls. For mult-lingual conferences, the main emphasis was on separation of the channels. This is usually achieved by hard-wired connections with limited frequency response to the range most important for spech intelligibility. The systems for the hard-of-hearing have also limited frequency response, a philosophy inherited from hearing aids. The priority here was adequate gain allowed by the system, provision to couple with personal hearing aids, and sometimes flexibility in seat selection. The modern listening systems installed in public places should serve both the general public and hard-of-hearing listeners. They are designed for both speech and music transmission and quality of sound becomes the primary objective. The authors have examined several listening systems installed in the New
York City and Washington, D. C. areas and will share some observations which can be helpful for the designers of the systems. [Work supported by NIH.]

$$
\text { 11:40 }
$$

J3. Near and farfield transfer-function technique for reverberation room response studies. W. T. Chu(Division of Building Research, National Research Council Canada, Ottawa, Ontario, Canada, K1A OR6)

The current qualification procedure for discrete frequency measurements in reverberation rooms is difficult and time-tenuming. The possibility of using the transfer function between a near-s microphone close to the speaker and another microphone in the reverterant field with narrow-band random noise excitation has been explored. Preliminary results indicate that this technique can be a very attractive alternative. It is fast and does not require any near-field measurement of the speaker in an anechoic room. 


\title{
Session K. Underwater Acoustics II: Acoustic Remote Sensing of the Ocean
}

\author{
William A. Kuperman, Chairman \\ Code 320, Naval Ocean Research and Development Activity, NSTL Station, Mississippi 39529
}

Chairman's Introduction-10:30

\section{Contributed Papers}

\begin{abstract}
K1. Global measures of resolution in acoustic tomography. Ronald New, Thomas J. Eisler, Denise Calderone, and David L. Porter (Systems Analysis Staff, NOAA, 6010 Executive Boulevard, Rockville, MD 20852)

We have previously reported on applications of the Backus-Gilbert theory to the problem of Ocean Acoustic Tomography [New et al., J. Acoust. Soc. Am. Suppl. 1 70, S38 (1981)]. This theory directly provides estimates of two important elements of system performance, viz., resolution and variance. In this paper, we concentrate on the former. Resolution of a pointwise estimate of the sound speed anomaly is fully described by a resolution kernel which, when integrated with the actual values of the unknown function, produces the estimate. This kernel is a system property; its suitably defined width is used to define resolution length at a point. We consider a horizontal ocean slice and define a global measure of resolution to be the area within which a given resolution criterion is satisfied. The shape and size of such regions have been calculated for various tomographic configurations. The behavior of this global measure as a function of number of moorings suggests that many more moorings are needed for resolution of mesoscale phenomena than had previously been thought!
\end{abstract}

\section{$10: 50$}

K2. Error measures for tomographic estimates of sound velocity. Denise Calderone, Ronald New, David L. Porter, and Thomas J. Eisler (Systems Analysis Staff, NOAA, 6010 Executive Boulevard, Rockville, MD 20852)

Inversion of perfect (error-free) tomographic data produces an imperfect estimate of the sound speed anomaly. The estimate is in error because the unknown function is sampled by a finite number of rays. This imperfection of the measuring system can be interpreted either in terms of resolution or in terms of bias error. In certain instances, the bias interpretation offers significant advantages, particularly when one wishes to quantify the error in the estimate. In this paper, we develop the latter interpretation. Our treatment employs various moments of the resolution kernels of the Backus-Gilbert theory. Methods for calculating bias error bounds as combinations of constants, dependent only on the tomographic configurations, and bounds on the higher order derivatives of the estimated function will be shown. Calculated bounds for a sample configuration will be presented. Use of these bias constants in comparisons of configuration performance will also be discussed.

\section{1:05}

K3. Remote sensing of environmental parameters. Paul G. Nadeau (Naval Underwater Systems Center, Newport, RI 02840) and Richard Nowak (Woods Hole Oceanographic Institute, Woods Hole, MA 02543)

This paper describes a prototype environmental parameter sampling system and the problems it is intended to address. Increasingly sophisticated sonar performance models are being developed in support of ad- vanced sonar system. A graphic portrayal of the simulated performance of a notional sonar system is presented as an example of the sensitivity of a simulation to the input environmental parameters including the volume scattering profile. The prototype environmental sampling system is configured to provide an estimate of the volume scattering profile. Operationally, this profile is not available to simulation users. The prototype environmental parameter sampler will be used to demonstrate how simulation errors due to this critical environmental input can be eliminated. The use of the microcomputer based prototype hardware as a basis for estimating other environmental parameters will also be presented. [Work supported by NAVSEA.]

\section{1:20}

K4. Transverse Doppler acoustic current meters. Thomas L. Clarke (Ocean Acoustics Laboratory, AOML, 4301 Rickenbacker Causeway, Miami, FL 33149)

Limitations of existing profiling acoustic current meters are examined. The characteristics of both single pulse processing and pulse to pulse coherent processing force severe compromises in performance. A new transducer geometry is proposed that, in conjunction with coherent processing, relaxes these constraints. The velocity component perpendicular to the beam is measured by utilizing the differential or transverse Doppler shift between the signals received by two spatially separated transducers. Examples are given of the application of this technique to a variety of flow regimes, and experiments designed to prove the feasibility of this technique are outlined.

\section{1:35}

K5. Monitoring strategies for acoustic remote sensing of the Florida Current. D. R. Palmer, L. M. Lawson, and Y. H. Chong (Ocean Acoustics Laboratory, NOAA/AOML, 4301 Rickenbacker Causeway, Miami, FL 33149)

In designing an acoustic system of monitoring the mass transport of the Florida Current, one is immediately faced with two, intimately related, problems. First, how does one determine which source/receiver configuration is optimal? And, second, what role does supplementary observations from nonacoustic systems play in the design and use of an acoustic system? This paper reports on our progress in developing a methodology for answering questions of this type. We describe an observational system, acoustic and/or nonacoustic, in terms of its "sampling region," i.e., the portion of the cross-sectional area of the Straits that it monitors. A very fast, specialized ray-tracing computer program is used to determine the sampling region for an acoustic system. Within the sampling region a system is assumed to provide an estimate of the current. Given these estimates, linear optimization theory is used to obtain an estimate for the transport. The linear coefficients which appear are determined by requiring that the mean-square error in the transport be a minimum. This minimum error, which serves as a measure of performance for the system, depends on the sampling region and sampling errors as well as on the environment through the covariance matrix for the current fluctuations. 
11:50

K6. Acoustic observations of large internal soliton waves at the Sulu Sea. John J. Tsai, John R. Proni, Julius Craynock (Ocean Acoustics Laboratory, NOAA/AOML, 4301 Rickenbacker Causeway, Miami, FL 33149), John R. Apel (Applied Physics Laboratory, Johns Hopkins University, Laurel, MD 20810), and James R. Holbrook (NOAA/ PMEL, Seattle, WA 98105)

Landsat imagery taken over the Sulu Sea, Philippines, reveals the existence of very long, soliton-like internal wave pockets of semi-diurnal periods. An experiment was conducted in May 1980 to test the hypothesis of tidal generation, and to parameterize these soliton waves by obtaining additional measurements using acoustic remote sensing techniques as well as conventional methods. In this paper, we compare wave characteristics from various measurements, and present different wave forms due to ship speed change with respect to the wave velocity. Effects of deep scattering layer on the acoustic measurements are discussed, using computerdigitized backscattering intensity contour.

\section{2:05}

K7. Deduction of bottom reflection phase change using normal modes. T. C. Yang (Code 5123, Naval Research Laboratory, Washington, DC 20375)

For discrete normal modes in shallow water, the mode characteristic equation is known to depend on the phase change of bottom reflection. Precise measurement of mode time-frequency dispersion has enabled us to deduce the phase change using just the shot data. We discuss the method and present the phase-change data for grazing angles between $9^{\circ}$ to $10.4^{\circ}$.
This proves to be a useful technique since direct experimental measurement of phase change is extremely difficult. Past work which investigated the bottom properties have concentrated on the reflectivity coefficients We find that the phase-change information can also shed light on the acoustic properties of the bottom. It is hoped that this technique for measuring the phase change will be utilized to gain further understanding of the ocean bottoms.

K8. The generation and measurement of $\mathrm{CO}_{2}$ laser-induced acoustic pulses in sand. G. Daniel Hickman and John A. Edmonds (Applied Science Technology, Inc., 2317 Thomas Drive, Panama City, FL 32407)

A pulsed $\mathrm{CO}_{2}$ laser (1- $\mu$ s pulse width, 10-J pulse energy) when focused to a $1-\mathrm{cm}^{2}$ spot on the surface of sand generated an acoustic field in the sand. The sound pressure level (SPL) measured with a hydrophone $1 \mathrm{~cm}$ below the surface was $173 \mathrm{~dB}$ re $1 \mu \mathrm{Pa}$ with a frequency spectrum $<3 \mathrm{kHz}$. Focusing this same laser on a water surface yields equivalent values in water of $200 \mathrm{~dB} r e l \mu \mathrm{Pa}$ and $25-30 \mathrm{kHz}$, respectively. Sound velocities in sand ranged between 145-184 $\mathrm{m} \mathrm{sec}^{-1}$ depending on the dryness of the sand. When the acoustic pulses were generated in a few $\mathrm{cm}$ of water covering the sand, the sound velocities measured in the sand increased to 610$750 \mathrm{~m} \mathrm{~s}^{-1}$ while the spectrum was composed of both the high frequency water spectrum and the low-frequency sand spectrum. Acoustic pulse measurements were made to sand depths of $40 \mathrm{~cm}$. In addition, pulses were detected after reflection off a metal plate at a depth of $45 \mathrm{~cm}$. A discussion will be given of possible remote sensing applications. [Research supported by ONR, Code $422 \mathrm{CS}$.] 


\title{
Session L. Physiological Acoustics II, Psychological Acoustics II, Noise II, and Physical Acoustics II: Modern Techniques of Vibration Measurement
}

\author{
Shyam M. Khanna, Chairman \\ Department of Electrical Engineering. Columbia University, New York, New York 10027 \\ Chairman's Introduction-1:30 \\ Invited Papers
}

$1: 40$

L1. ESPI-The ultimate holographic tool for vibration analysis? Ole J. L 6 kberg (Department of Physics, The Norwegian Institute of Technology, 7034-NTH, Trondheim, Norway)

Electronic speckle pattern interferometry-ESPI-may be described as image holography, where the TVtarget has replaced the the photographic film as recording medium while the optical reconstruction is simulatad by videoprocessing and display. ESPI can do similar interferometric measurements as holography, but the technique is especially suitable for measurements on vibrating objects. Here the object's vibration mode is displayed as easily interpretable fringe patterns across the monitor image without time delay and at a high repetition rate. ESPI permits measurements on difficult, unstable objects in adverse environments. Using optical phase-modulation techniques in ESPI, we obtain an extended measuring range (lower-upper limits 0.01-10000 NM), separate contours of constant amplitude and phase, dynamic display of the vibration cycle and direct comparison between vibrating objects. The ESPI technique has been applied in various industrial and biomedical projects, which will be described. The general properties outlined above together with some samples of vibrating objects will be illustrated by a short movie.

\section{2:30}

L2. Heterodyne interferometry and holography for vibration measurements. René Dändliker (Institut de Microtechnique de l'Université, CH-2000 Neuchâtel, Switzerland)

Two frequency or heterodyne interferometry transforms the optical interference phase into the phase of an electronically detectable beat-frequency signal. This allows to use powerful signal processing for increased sensitivity far below the optical wavelength $\lambda$ and high temporal resolution, especially for vibration analysis. The principle and the properties of this heterodyne technique will be illustrated by presenting laser interferometry for displacement and velocity measurements. With an extended version [R. Dändliker and J.-F. Willemin, Opt. Lett. 6, 165-167 (1981)] amplitude and phase of in-plane and out-of-plane microvibrations (amplitudes $1 \mathrm{~nm}$, spatial resolution $30 \mu \mathrm{m}$ ) at frequencies from $5 \mathrm{kHz}$ to $5 \mathrm{MHz}$ can be analyzed in real time. Experimental results for piezoelectric ultrasound transducers will be presented. Two-reference-beam holographic interferometry allows to use the heterodyne technique for accurate fringe evaluation and interpolation $(\lambda / 1000)$, since the two object fields are stored and accessible independently by the corresponding reference beam [R. Dändliker, Progress in Optics (North-Holland, Amsterdam, 1980), Vol. 17, pp. 1-84.]. For vibration analysis, however, the object has to be recorded holographically by two or more coherent light pulses, synchronized to the object movement, using pulsed lasers or stroboscopic light modulation. Experimental results of deformation measurements by heterodyne holographic interferometry will be presented. The strength and weakness of this technique applied to vibration analysis will be discussed in particular.

L3. Holographic measurement of tympanic membrane vibrations. G. von Bally (Medical Acoustics and Biophysics Laboratory, ENT-Clinic, University of Münster, Kardinal-von-Galen-Ring 10, D-4400 Münster, West Germany)

Different holographic methods have been used for high resolving, contactless, $3 \mathrm{D}$ analysis of tympanic membrane vibrations [G. von Bally, Ed., Holography in Medicine and Biology (Springer, Berlin, 1979)]. Most experiments have been performed in-vitro for basic research on the transfer function of the middle ear. We have chosen double-exposure holography using a $Q$-switched ruby laser system, a (vibration-) phase sensitive technique which allows applications in-vivo. For simultaneous sound presentation and holographic recording of the elicited tympanic membrane vibration through the outer ear canal in humans, special closed acoustic systems were developed. Thus, it was possible to determine a vibration pattern in patients with regular middle ear status, which characteristicly depends on amplitude, frequency, and phase of the stimulus. Specific deviations from this "normal" vibration pattern were determined in investigations on patients with pathologically altered middle ear mechanics, proving the applicability of this method for diagnostic purposes. Increased feasibility for 
clinical use was achieved with flexible fiber optics for convenient positioning of the acousto-optical system and the use of otoscopic optics for easy optical access to the tympanic membrane. [Supported by Deutsche Forschungsgemeinschaft SFB 88/B3.]

4:10 to 4:40

Discussion

TUESDAY AFTERNOON, 9 NOVEMBER 1982

PINELLAS ROOM, 2:00 TO 5:00 P.M.

\title{
Session M. Architectural Acoustics III: Open Forum on Contemporary Issues in Architectural Acoustics
}

\author{
William J. Cavanaugh, Moderator
}

Cavanaugh Tocci Associates Inc., 18 Main Street, Natick, Massachusetts 01760

\begin{abstract}
Among the issues slated for discussion are what guidelines should architectural acousticians follow in dealing with music critics, the public at large and fellow professionals? and, are we applying all we know towards producing better buildings acoustically?... if not, why not? Several colleagues both within and outside of the acoustics community have been invited to outline various issues and lead what we hope will be a lively dialogue with all attending. Those wishing to present an issue for discussion should contact the Moderator at least 24 hours in advance of the session.
\end{abstract}

\author{
Moderator's Introduction-2:00
}

Invited Paper

2:05

M1. Acoustics of EPCOT. M. Long (Marshall Long/Acoustics, Santa Monica, CA 90404)

Walt Disney Productions and their engineering arm WED Enterprises have undertaken to build the Experimental Prototype Community of Tomorrow (EPCOT), the largest theme park complex in the world. This park recently opened in Orlando, Florida and features 17 pavilions in two groups, Future World and World Showcase. The project which will cost over a billion dollars to build has a number of interesting and unique acoustical features. The author who served as consultant on 6 of 7 Future World Pavilions and 8 of 10 World Showcase Pavilions will review several of the most interesting acoustical aspects of this monumental facility. 


\title{
Session N. Engineering Acoustics II: Transducers
}

\author{
Allan C. Tims, Chairman \\ Naval Research Laboratory, P.O. Box 8337, Orlando, Florida 32856
}

Chairman's Introduction-2:00

Invited Paper

2:05

\begin{abstract}
N1. Pragmatic aspects of underwater transducer design. Theodore J. Mapes (Naval Underwater Systems Center, New London, CT 06320)

The majority of failures experienced by underwater transducers are caused by deterioration of the materials which are employed to protect the active elements from the hostile effects of the seawater environment. In many cases such failures result from a poor choice of materials or from lack of consideration of the compatibility of the materials with themselves and with their environment. This paper reviews several case histories which highlight poor design practice in materials application. Guidelines for materials selection are promulgated in the hope that knowledge of past mistakes will provide insight towards avoiding repetition of these failures in future underwater transducer designs. Both organic and metallic materials are included in the study.
\end{abstract}

\section{Contributed Papers}

\section{2:35}

N2. A reliability model for predicting electric flashover failures of transducer ceramics. Lynn P. Browder (Naval Research Laboratory, Underwater Sound Reference Detachment, P.O. Box 8337, Orlando, FL 32856)

The electrical breakdown of sonar transducers has been a matter of great concern in recent years. Transducer replacement is generally very expensive, so high reliability is required of each unit in order to reduce lifecycle cost and to improve sonar system readiness. Since it is advantageous to be able to mathematically predict the life time of a transducer in its design stage, a reliability model has been developed by considering electric flashover failures at the ceramic/gas interface. The applied voltage that causes flashover may now be calculated as a function of time-to-breakdown in a transducer. Based on the voltage endurance test results on ceramic samples, the effects of physical parameters such as creepage distance and frequency, and environmental conditions including temperature, gas pressure, air contamination, and water vapor were determined. A random factor was also included to approximate the random variation of flashover voltage levels due to the combined effect of all the other factors. In addition to presenting the model itself, potential application to transducer reliability estimation will also be discussed.

\section{2:50}

N3. Temperature effects in piezoelectric ceramic-stack resonators containing cement joints. C. L. Goodhart (Transduction Sciences Branch, Code 7122, Naval Ocean Systems Center, San Diego, CA 92152)

Underwater acoustic transducers often employ a stack of piezoelectric rings that are cemented together, face to face, as the driving element. Recently, the performance of such a transducer was observed to seriously deteriorate when subjected to somewhat elevated temperatures. The source of the problem was determined to be the effects of elevated temperature on the cement joints. This paper discusses the temperature-related effects in cement joints on the performance of ceramic stack resonators.
Measurements of cement-joint compliance and loss factor as a function of temperature are presented. The behavior of ceramic-stack resonators resulting from temperature effects in the cement joints is examined using results from both theoretical models and experimental measurements. It is shown that as temperature increases, a corresponding increase in the cement-joint compliance and loss factor results in detuning, decreased electromechanical coupling and increased heating (which further provokes the problem) in the resonator. Methods of modifying the cementjoint characteristics in order to reduce the ill effects of temperature are discussed. [Work supported by NAVSEA 63X.]

\section{3:05}

N4. Low temperature diffusion bonding of transducer ceramics. Stephen C. Thompson, Andrew A. Lux, and John J. Gray (Gould Inc., Ocean Systems Division, 18901 Euclid Avenue, Cleveland, $\mathrm{OH} 44117$ )

A metallic diffusion bonding process has been developed to bond piezoelectric ceramics into stacks for use in sonar transducers. The assembly process starts with stacking the ceramics to be bonded alternately with layers of a diffusing material. This material is a thin shim of a four component alloy of indium, silver, lead, and tin. This alloy bonds by diffusing uniformly into the surface of the fired-on silver electrode on the ceramic face. A solid silver electrical lead may also be included in the stack. Surface preparation of all pieces is critical as minute amounts of surface oxidation or other contaminants will yield unacceptable bond strength. The stack is placed under moderated compression in an elevated temperature environment for the diffusion process. The temperatures involved are well below the Curie point for the ceramic material and give minimal degradation of the electrochemical coupling constant of the material. Tensile testing shows the diffusion bond to have higher strength than the ceramic material-The ceramic will fracture before the bond breaks. Preliminary results show the metallic bond to have less compliance and a lower loss factor than conventional epoxy bonding methods. Further tests are underway to better determine the bond mechanical properties. 
occupies the bandwidth of the information signal. It is a simple matter to

N5. Optimum tranisducer parameters for broadband applications. William J. Marshall, Jr. (Sanders Associates, Inc., Surveillance Systems Division, 95 Canal Street, Nashua, NH 03061)

A general method for making top-level sizing and design decisions for broadband transducers is presented. The realm of application is that of body force transducers having small radiating area at resonance. By "broadband applications" we mean that the system requirement driving the design is the shape and magnitude of the constant-voltage acoustic spectrum. The design method yields the optimum $Q_{m}$ and resonance frequency to meet the specified acoustic performance for given constraints on transducing material. The theory is illustrated by applying it to two common spectral shapes: white and pink noise of arbitrary bandwidth.

\section{3:35}

N6. Expendable sonobuoy hydrophone. T. A. Henriquez and A. C. Tims (Naval Research Laboratory, Underwater Sound Reference Detachment, P. O. Box 8337, Orlando, FL 32856)

A shock-hardened, low-cost pressure sensitive hydrophone has been developed for applications in expendable air-deployed passive sonar systems. The hydrophone is completely functional after sustaining the shock from an underwater explosion equivalent to $5 \mathrm{~kg}$ of dynamite at a distance of $3 \mathrm{~m}$. A novel preamplifier integral to the hydrophone has very low power consumption and self-noise. The preamplifier also has a very low output impedance which is necessary to drive long cables. The design is easily assembled and is oriented toward mass production to provide large quantities at very low cost.

\section{3:50}

N7. Development of a dual mode transducer array. Richard L. Schmidt and Jan F. Lindberg (Naval Underwater Systems Center, Newport, RI 02840)

A task was initiated to develop a sonar system serisor which permitted both conventional and parametric beam formation. Available space constraints and required acoustic power densities demanded that the conventional and parameteric signals be generated from the same transducer array. Extensive studies are reported that document simulations and experiment's undertaken to determine the best parametric primary frequencies. A dual-mode transducer was developed to generate both conventional and parametric signals. The tranisducer has the capability of high power transmission at two separate frequencies almost 2.5 octaves apart with broad bandwidth at both frequencies. The low-frequency transducer is a traditional Tonpliz with its head mass comprised of a multitude of nodally mounted high-frequency transducers which generate the parameteric sig. nal. The nodal plate of the high-frequency transducer permits the lowfrequency transducer to transmit acoustic energy to the medium without transversing the acoustic pressure release of the high-frequency transducers. Data are shown of the performance of a small partial array of these transducers.

\section{4:05}

N8. A monitor hydrophone system for accurate measurement of sound pressure levels produced by deep towed sources. Ira J. Bush and Ronald N. Demetres (Naval Research Laboratory, Underwater Sound Reference Detachment, P.O. Box 8337, Orlando, FL 32856)

The only way to accurately determine the sound pressure level of a towed high power acoustic source is to place a calibrated standard at a fixed distance in close proximity to the source. From a practical standpoint, the source and monitor hydrophone must share the same electromechanical cable. Crosstalk results from cable sharing because the drive signal to the high power source and the return monitor signal are of the same frequency and contained within the same cable. For the case of most tow cables, shielding between the two signals is almost nonexistant. The resulting cross-talk obfuscates the output and usefulness of the monitor. If the return signal is frequency modulated, the cross-talk signal no longer filter out the cross-talk signal before the information signal is locally demodulated. Synchronous detection is utilized to insure accuracy, linearity and immunity to carrier drift. A prototype system has been successfully tested at calibration facilities and out at sea demonstrating accurate measurements for both electrodynamic and piezoelectric sources. [Work supported by NRL.]

\section{4:20}

\section{N9. Abstract withdrawn.}

\section{4:35}

N10. Development of a hydraulically powered underwater acoustic projector for use in the range of 1 to $10 \mathrm{~Hz}$. George D. Hugus, III (Naval Research Laboratory, Underwater Sound Reference Detachment, P.O. Box 8337, Orlando, FL 32856)

Development of a hydraulically powered acoustic projector with a minimum source level of $160 \mathrm{~dB}$ re: $1 \mu \mathrm{Pa}$ at $1 \mathrm{~m}$ over the 1 to $10 \mathrm{~Hz}$ range will be presented. This projector will be used at the Underwater Sound Reference Detachment for low-frequency underwater acoustic testing. Design parameters and their relation to the unique capabilities of hydraulic power for this low-frequency application will be discussed. Construction details, and acoustic as well as mechanical performance will also be presented. [Work supported by Naval Sea Systems Command, Code 63R.]

\section{4:50}

N11. Acoustical matching of a traveling wave heat engine. P. H. Ceperley (Department of Physics, George Mason University, Fairfax, VA 22030)

A traveling wave heat efigine is a thermally driven acoustical amplifier which uses a differentially heated regenerator as the gain element. However, because it amplifies only the volumetric flow component of an acoustical wave and not the pressure component, the impedance of the wave changes in going through the device, thereby complicating the impedance matching to the input and output wave guiding tubes. This paper derives expressions for this impedance change and discusses possible matching methods. It also presents some experimental verification of both. Other relevent matching considerations such as impedance variations due to temperature and tubing dimension changes are discussed. Earlier papers by this author introduced the traveling wave heat engine and calculated its gain and efficiency [P. H. Ceperley, J. Acoust. Soc. Am. 66, 1508-1513 (1979)]

\section{5:05}

N12. Analytical formulation and experimental study of the properties of piezopolymer transducers using shell-or membrane-dome structures. P. Ravinet, F. Micheron, D. Guillou, and C. Claudepierre (ThomsonCSF, Central Research Laboratory, B.P. 10, 91401 Orsay Cedex, France)

Dome shaped piezopolymer transducers can be considered as membrane or shell structures, depending on the thickness of the thermoformed film. We report an analysis of the mechanical behavior of such structures, taking into account both the extensional and flexural effects, and leading to a formulation of their acoustic mass and compliance and of the frequency of their first resonance mode. The microphone sensitivity and the transmitter efficiency are then, respectively, derived from the stress field induced by an applied pressure and from the relative change of radius of curvature due to an applied electrical field. These predictions are more specifically applied to the optimization of telephone microphones and receivers. It is shown that the product of the microphone sensitivity by the square of the resonance frequency and the radius of curvature is a function of material parameters only. Maximal receiver efficiency is ascribed to the piezoelectric coupling factor of the material. Experimental results will be presented, concerning such transducers using home made piezopolymer $\mathrm{PVF}_{2}$ in shell or membrane structures. [Work sponsored by DAII.] 


\title{
Session O. Noise III: Review of A-Weighting for Noise Assessment
}

\author{
Daniel L. Johnson, Chairman
}

Aerospace Medical Research Lab., AMRL/TS, Wright-Patterson AFB, Ohio 45433

\author{
Chairman's Introduction-2:00
}

\section{Invited Papers}

01. Historical development of the use of A-weighting. James H. Botsford (Howard Engineering Company, 3456 Altonah Road, Bethlehem, PA 18017)

The original writers of the specifications for American sound level meters wished to provide a standard instrument that would indicate the loudness of sounds as perceived by the normal human ear. As loudness varies with the sound pressure level and frequency of a sound, three frequency weighting networks were specified to mimic the response of the ear at the loudness levels of 40,70 , and approximately 100 phons. The first of these was designated the $\mathrm{A}$-weighting and the last was called the $\mathrm{C}$-weighting, which was a flat response. When the rules given for using the weighting networks were followed, the sound level meter indicated very accurately the loudness levels of pure tones but not broadband noises. For measuring noises more precisely, special rules were developed by sound level meter users for combining the readings obtained using two or more of the weighting networks, particularly the $\mathrm{A}$ - and $\mathrm{C}$-weighting networks. Long experience with estimating human responses to noise from sound level meter readings has led to the general use of the A-weighting network alone for many purposes.

O2. A-weighting and the rating of auditory hazard. G. Richard Price (U.S. Army Human Engineering Laboratory, Aberdeen Proving Ground, MD 21005)

Considering the complexity of the alternatives, it is easy to understand the reasons that evaluation procedures using A-weighting have gained acceptance. On the other hand, it may seem suprising that this specific weighting, not originally intended for rating hazard, should have proved so useful in that role. From a theoretical viewpoint it can be argued that the shape of the curve is a function of physiological mechanisms that also underlie hearing loss. This paper will discuss these mechanisms as they are becoming known and the implications they have for the use of A-weighting with special emphasis on the noise research conducted in the last decade.

\section{2:55}

O3. Frequency weighting for community response and annoyance. Karl S. Pearsons (Bolt Beranek and Newman Inc., Canoga Park, CA 91305)

Subjective evaluations of various noises have been studied over the years by many researchers. The results of these studies have provided various schemes for predicting annoyance, loudness, or noisiness of the noises using objective measures. A review of some of the pertinent studies will be presented including evaluations of some of the measures in current use. A discussion of community response studies will also be presented which indicates the variety of frequency weightings which have been used in past research. Information on situations which may require special weightings such as those which include impulse noise, tones, or high-frequency noise will also be presented. Mention will also be made of an approach which considers the ambient noise as well as the intruding noise.

O4. Frequency weighting for the assessment of speech interference. J. C. Webster (Communication Research Department, National Technical Institute for the Deaf, Rochester Institute of Technology, Rochester, NY 14623)

All known frequency weighting systems for evaluating the effects of noise on speech discrimination will be reviewed and evaluated. These will include the articulation index (AI); the speech interference level (SIL); speech, noise, and alternate noise contours (SC, NC, NCA); the speech transmission index (STI) and modulation transfer function (MTF). Also there will be a discussion of the problems associated with measuring speech level (vocal effort) and with measuring those aspects of noise that affect talking and/or listening levels and the discriminability of spoken language. It has been found that the equivalent noise level $\left(L_{\text {eq }}\right)$ measured in $\mathrm{dB}(\mathrm{A})$ is a good measure of the effects of time varying noise on speech intelligibility (discrimination). Therefore it will be 
suggested that the A-weighted level of noise, which is not the best speech interference measure for steady-state wideband noises, may be the best simple comprehensive measure of the speech interfering aspects of noise.

\title{
$3: 45$
}

O5. Frequency weighting to assess sleep and classroom environments. Jerome S. Lukas (California Department of Health Services, Office of Noise Control, Berkeley, CA 94704]

Only a limited amount of specific data are available regarding the relative effectiveness of A-versus Cweightings in predicting the effects of noise on sleep or of noise on children in classrooms. These data will be reviewed, but suggest that with respect to sleep the results are equivocal. In the classroom, the single study available [Lukas, DuPree, and Swing, in press] suggests the C-weighted noise levels are more highly correlated (negatively) wth academic achievement that are A-weighted levels.

O6. A-weighting for assessment of highway noise impact. Charles E. Wilson (New Jersey Institute of Technology, 323 High Street, Newark, NJ 07102)

Exposure to transportation noise sometimes results in unacceptable noise levels inside residential and office buildings. Underprediction of intrusive noise can result from the frequency characteristics of building attenuation and from room effects when low-frequency tones are prominent at the source. The significance of A-weighting in relation to this problem is investigated through simultaneous measurements of indoor and outdoor noise.
07. An improved procedure for evaluating the annoyance of small arms ranges. George Luz (US Army Environmental Hygiene Agency, Aberdeen Proving Ground, MD 21010)

By combining annoyance data published in studies of small arms ranges in Sweden and Australia with other data on the acoustics and levels of small arms fire published in limited distribution Army and Navy technical reports, the author derives a computer program for predicting the impact and annoyance of small arms ranges firing ammunition up to 25 $\mathrm{mm}$. Copyright free copies of the computer program written in BASIC will be available for interested participants.

TUESDAY AFTERNOON, 9 NOVEMBER 1982

DADE AND FLORIDA KEYS ROOMS, 2:00 TO 5:05 P.M.

\author{
Session P. Physical Acoustics III: Relaxation, Interactions, and Propagation \\ F. Douglas Shields, Chairman
}

Physics Department, University of Mississippi, University, Mississippi 38677

Chairman's Introduction-2:00

\section{Contributed Papers}

\section{2:05}

P1. Self-de-excitation of water vapor: Monomer and dimer contributions. Allan J. Zuckerwar (NASA Langley Research Center, M/S 238, Hampton, VA 23665

Past efforts to interpret experimental data on the de-excitation rate of the $v_{2}(1 \rightarrow 0)$ vibrational mode of water vapor have been thwarted because of the attempt to fit the data to a single relaxation time. In a phenomenological theory proposed here the de-excitation takes place by means of two parallel reactions: (1) the conventional collisional de-excitation of the monomer and (2) a two-step reaction involving association and re-dissociation of the dimer. The resulting relaxation equation yields two coupled relaxation times. In pure $\mathrm{H}_{2} \mathrm{O}$ and in mixtures without $\mathrm{O}_{2}$, the reaction rate for dimer association is taken to be very slow, and the relaxation modes are effectively decoupled: the first corresponding to de-excitation of the monomer and the second to spontaneous dissociation of the dimer. In mixtures of $\mathrm{H}_{2} \mathrm{O}$-air and $\mathrm{H}_{2} \mathrm{O}-\mathrm{O}_{2}$, the association rate of the dimer is found to increase strongly, suggesting that $\mathrm{O}_{2}$ serves as an effective "chap- eron" for this reaction, and the relaxation modes of the monomer and dimer become strongly coupled. Fourteen sets of past data, representing a wide variety of test conditions and experimental methods, both acoustical and nonacoustical, are organized into three groups - each corresponding to a relaxation mode predicted by the theory.

\section{2:20}

P2. Relaxation mechanism for low-frequency sound absorption by boric acid in sea water. Robert H. Mellen (MAR, Incorporated, East Lyme, CT 06333)

Investigation of the low-frequency boric acid relaxation in sea water by the resonator method shows that the mechanisms is an exchange reaction with the carbonate system, i.e., $\mathrm{B}(\mathrm{OH})_{3}+\mathrm{CO}_{3}^{-2}$ $\rightleftharpoons \mathrm{B}(\mathrm{OH})_{4}^{-}+\mathrm{HCO}_{3}^{-}$. However, the absorption is small unless calcium is present; then the system can be modeled as a complex exchange involving the ion pairs $\mathrm{CaCO}_{3}^{\circ}$ and $\left.\mathrm{CaB} / \mathrm{OH}\right)_{4}^{+}$. Theory and supporting experimental results are presented. [Work supported by DARPA.] 
measured earlier in a similar way for W [F. Douglas Shields, J. Chem. Phys. 72, 3767 (1979); 76, 3809 (1982); 76, 3814 (1982)]. Exposing the flashed surface to $\mathrm{O}_{2}$ raised the combined values of TMAC + EAC to 1.28 for $\mathrm{Ne}$ and 1.00 for $\mathrm{He}$. Flashing the surface at $1100 \mathrm{~K}$ removed some of the $\mathrm{O}_{2}$ and flashing at $1400 \mathrm{~K}$ restored the original $\mathrm{AC}$ values. In contrast to this, exposure to $\mathrm{N}_{2}$ did not change the surface. Exposure to $\mathrm{CO}$ raised the $\mathrm{AC}$ values but not as much as $\mathrm{O}_{2}$ but the $\mathrm{CO}$ was removed by the $1100 \mathrm{~K}$ flash. AC values have also been obtained for $\mathrm{N}_{2}$ on $\mathrm{Pt}$, for $\mathrm{O}_{2}$ on $\mathrm{O}_{2}$ on $\mathrm{Pt}$ and for $\mathrm{CO}$ on $\mathrm{CO}$ on $\mathrm{Pt}$. threshold of water with concentration of the polymer additives polyethylene oxide and guar gum. It was found that small amounts of these additives could significantly increase the cavitation threshold. A theoretical model, based upon nucleation of a gas bubble from a Harvey-type crevice in a mote or solid particle, is developed that gives good agreement with the measurements. The applicability of this approach to an explanation of cavitation index reduction in flow-generated or confined jet cavitation, when polymer additives are introduced, is discussed. [Work supported in part by the ONR and the NSF.]

\section{2:50}

P4. The effect of pulse length on transient cavitation inception. Ronald A. Roy and Lawrence A. Crum (Physical Acoustics Research Group, Department of Physics, University of Mississippi, Oxford, MS 38677)

Most ultrasonic systems used for diagnostic purposes employ short acoustic pulse rather than cw operation in order to accomplish imaging or Doppler scanning. It is also commonly thought that these short-pulse systems, although driven at high acoustic pressure amplitudes, do not induce cavitation effects due to the low duty cycle of the ultrasound. Although stable cavitation typically requires cw operation for its effects to be present, transient cavitation can theoretically occur in pulse lengths as short as one cycle. We have developed a detection scheme utilizing sonoluminescence to observe the cavitation produced by pulses of varying length and repetition rate. Normal detection methods that examine noise emitted by the collapsing cavity can not be used in short-pulse excitation due to the high spectral content of the pulse itself. We have observed transient cavitation effects (sonoluminescence) in pulse lengths as low as a few tens of cycles and are modifying our apparatus to go to even shorter pulses. [Work supported in part by the ONR and the NSF.]

3:05

P5. Stress waves induced in graphite-epoxy composite structures by current pulses. Jacques R. Chamuel (The Charles Stark Draper Laboratory, Inc., 555 Technology Square, Cambrige, MA 02139)

A technique to generate ultrasonic waves in graphite-epoxy composite structures using electrical current pulses flowing in the structure is described. The difference between the thermal expansion coefficients of graphite and epoxy create large stress waves in response to localized heating by current pulses flowing in the individual graphite fibers. Experimental results are presented demonstrating the applicability of the method to nondestructive testing problems. The anisotropic elastic and electrical properties of a T-300 graphite/DER-332 epoxy composite plate were determined. In the experiments, a piezoelectric receiver transducer was used to detect the thermoelastic waves in the composite plate. Distinct ultrasonic waves were detected with $20 \mu \mathrm{sec}$ current pulses below $50 \mathrm{~mA}$.

P6. Acoustical measurement of energy and momentum accommodation coefficients on polyerystalline platinum. F. Douglas Shields (Department of Physics and Astronomy, The University of Mississippi, University, MS 38677)

The acoustical method of measuring energy (EAC), and tangential momentum (TMAC) accommodation coefficients [F. Douglas Shields, J. Chem. Phys. 62, 1248-1252 (1975)] has been used to measure the accommodation coefficients of $\mathrm{Ne}$ and $\mathrm{He}$ on vacuum flashed $\mathrm{Pt}$ and on $\mathrm{Pt}$ after exposure to $\mathrm{O}_{2}, \mathrm{~N}_{2}, \mathrm{CO}$, and $\mathrm{CO}_{2}$. Vacuum flashing the $\mathrm{Pt}$ at $1400 \mathrm{~K}$ in the presence of misch metal getter resulted in a surface that was reproduced a number of times after exposure to various adsorbants. The combined value of TMAC + EAC for this surface was $0.80 \pm 0.04$ for $\mathrm{Ne}$ and $0.64 \pm 0.04$ for $\mathrm{He}$. These values are significantly greater than values 
dependence of wave speed normal to the stratification and the alternation of pass and stop bands for such propagation.

$4: 20$

P10. A variational method for wave propagation and scattering in discrete random media: The coherent field for scalar waves. Robert $\mathbf{H}$. Andreo (Applied Physics Laboratory, Johns Hopkins University, Johns Hopkins Road, Laurel, MD 20707)

The propagation and scattering of scalar waves in a random medium of particles is analyzed from a variational point of view. Using an arbitrary set of basis functions, multiple scattering equations are obtained for the multipole coefficients in terms of the single-particle transition matrix elements and the matrix elements of the Helmholtz-Green function. Comparison is made with the method of moments and the method of weighted residuals. Closure hypothesis lead to algebraic equations for the coherent scattered field and integral equations for the coherent exciting field. With suitable assumptions on the pair correlation function, dispersion relations can be obtained which characterize the effective macroscopic random medium in terms of an experimentally measurable phase velocity and attenuation coefficient. Examples are discussed, with emphasis on the physical role of the pair correlation function and the mathematical role of the closure hypothesis. [Work supported by NAVSEA and ARO.]

\section{4:35}

P11. Frequency dependence of the velocity and attenuation of sound waves propagating in a fluid containing a dense suspension of particles. $V$. K. Varadan, Y. Ma, and V. V. Varadan (Wave Propagation Group, Department of Engineering Mechanics, The Ohio State University, Columbus, OH 43210)

Acoustic waves propagating through a suspension of particles in a fluid are dispersed and attenuated due to two reasons-geometric disper- sion or multiple scattering as well as inherent losses if any associated with the particles or the host fluid. A self-consistent multiple scattering theory is used incorporating the $T$ matrix of the individual particles to characterize their response and a configurational averaging procedure to characterize the statistics of the distribution. Numerical results are obtained for the phase velocity and attenuation of the coherent wave in such a system as a function of the frequency, size, shape, and distribution of the particles of the suspension. These results will be compared with single scattering approximation as well as more approximate methods. The results are of interest in the study of ocean bottom sediments and biological applications.

\section{4:50}

P12. Resonance dependence of the dynamic effective properties of particulate composites. G. C. Gaunaurd and H. Überall (Naval Surface Weapons Center, R43, White Oak, Silver Spring, MD 20910)

We have developed a general deterministic resonance theory for the effective dynamic properties of elastic (or viscoelastic) solids containing ensembles of randomly distributed solid spherical inclusions not necessarily of the same sizes. The method follows our earlier procedure for fluid inclusions [J. Acoust. Soc. Am. 71, 282-295 (1982)] which is generalized here for solid inclusions of known size distributions. We present a summary of the theory and numerous displays of calculations for the effective shear speed, the effective (sound) speed of compressional waves, and the effective attenuation versus $k a$ at known concentrations $\Phi$, or versus $\Phi$ at various values of $k a$. The current theory contains nearly all deterministic results available in the earlier literature and it compares quite favorably, at least within the "acoustical branches," to the only set of dynamic measurements in the recent literature [i.e., V. Kinra and A. Anand, Int. J. Solids Struct. 18, 367-380 (1982)] which extends sufficiently beyond the long-wavelength regime to permit comparisons. [H. Überall is also at Catholic University, Washington, DC and is additionally supported by ONR.]

\title{
Session Q. Shock and Vibration II: Active Systems for the Reduction of Vibration and Sound and Advanced Instrumentation
}

\author{
Frank P. Finlon, Chairman
}

Applied Research Laboratory, Pennsylvania State University, P. O. Box 30, State College, Pennsylvania 16801

Chairman's Introduction-2:00

Invited Papers

2:05

Q1. Recent developments in active vibration suppression. Leonard Meirovitch (Department of Engineering Science and Mechanics, Virginia Polytechnic Institute and State University, Blacksburg, VA 24061)

In many mechanical systems, vibration is an undesirable effect, to be suppressed as expeditiously as possible. Traditionally, vibration suppression has been achieved by passive means, such as vibration absorbers, dampers, etc. In seeking more efficient vibration reduction, particularly for larger-order systems, there has been a recent trend toward active feedback control. Vibrating systems can be divided into lumped-parameter and distributed-parameter systems. Feedback controls for lumped-parameter systems can be designed by methods of modern control, such as pole allocation, optimal control, etc. In applying techniques of modern control to distributed-parameter systems, difficulties arise because modern control theory is essentially a discrete (-in-space) theory, not well suited for distributed systems. To circumvent this problem, the tendency has been to discretize (in space) the distributed system. This approach has the effect of imparting to the 
distributed system all the characteristics of a lumped system. A recently developed method, called the independent modal-space control method, is more faithful to the distributed model and is more suitable for the control of distributed systems.

2:35

Q2. Historical review and recent development of active attenuators. H. G. Leventhall (Atkins Research and Development, Woodcote Grove, Ashley Road, Epsom, Surrey KT18 5BW, United Kingdom)

Active attenuators are now about 50 years old. Their history has been one of disappointment, demise, and rediscovery, although modern electronics and computing has resulted in remarkable advances in recent years. Early practical applications were in the mid 1950's for the control of transformer noise and a wide range of applications was proposed by Olsen. Some of these are the ones which are being developed today. Following the developments in the 1950's, there was little progress for a further 10 years when theoretical advances were made by Jessel. Much of the modern interest in active attenuators can be traced to Jessel, who was the first to use a unidirectional source which gives absorption of sound rather than cancellation by interference. Subsequently, a different form of unidirectional source was proposed by Swinbanks and active attenuators have attracted considerable interest during the past 15 years. The current trend is to return to simple radiating systems and make electronic compensation for their deficiencies. At the present time, the basic Olsen type of monopole attenuator gives a good prospect for an inexpensive duct attenuator for low-frequency noise.

3:05

Q3. Current developments in automatic adaptive control of noise. Glenn E. Warnaka (Lord Corporation, 2000 West Grandview Blvd., P. O. Box 10038, Erie, PA 16514-0038)

Modern solid-state electronics and control theory allow active control of noise by the carefully controlled generation of out-of-phase cancellation signals from loudspeakers. Active control of noise is rather easily applied to the problem of noise in ducts where the waves are constrained to be one dimensional. However, with careful consideration of the acoustical parameters, active noise control can also be applied to two- and threedimensional problems.

\section{Contributed Papers}

\section{3;50}

Q4. Adaptive active sound control. John C. Burgess (Department of Mechanical Engineering, 2540 Dole Street, University of Hawaii, Honolulu, HI 96822)

Adaptive signal processing has been used to cancel echoes in telephone networks and to improve signal-to-noise ratios in measurement systems. Adaptive methods offer the potential for significant improvement over existing methods for active sound and vibration control. Adaptive methods can accommodate feedback with transport delay, operate over a broad frequency range, and adjust to uncontrollable system parameters such as changes in fluid velocity, fluid temperature, and component aging. A computer simulation shows feasibility for adaptive active sound control in a duct [J. C. Burgess, "Active adaptive sound control in a duct: A computer simulation," J. Acoust. Soc. Am. 70, 715-726 (1981)].
Q5. A new digital technique for the measurement of mechanical vibration. Gunnar Rasmussen (Brüel \& Kjaer, 18 Naerum Hovedgade, 2850 Naerum, Denmark)

The two-channel FFT analyzers have revolutionized the measurement technique used for mechanical measurements. Recursive digital filter technique is in general use within acoustics. However, the true real time capability, eliminating the use of window functions like f.ex. Hanning along with born logarithmic frequency scale, offers great advantages for the measurement of mechanical vibrations. The measurement of vibrational net power flow, vibration modes and stress intensity by mechanical transducers will be discussed.

\title{
Session R. Speech Communication II: Speech Recognition
}

\author{
Harry L. Hollien, Chairman
}

IASCP, 50 ASB, University of Florida, Gainesville, Florida 32611

\author{
Chairman's Introduction-2:00
}

\section{Contributed Papers}

This paper presents a new approach to text-independent speaker recognition. The technique, developed to perform with short unknown utterances, models the spectral traits of a speaker with multiple sub-models rather than using a single statistical distribution as done with previous approaches. The recognition is based on the statistical distribution of the

R1. Text-independent speaker recognition with short utterances. $K$. P. Li and E. H. Wrench, Jr. (ITT Defense Communications Division, 10060 Carroll Canyon Road, San Diego, CA 92131) 104th Meeting: Acoustical Society of America 
distances between the unknown speaker and each of the speaker models. Only frames that are close to one of the speaker's sub-models are considered in the recognition decision, so that speech events not encountered in the training data do not bias the recognition. The technique has been tested on a conversational data base. Models were generated using $100 \mathrm{~s}$ of speech from each of 11 male talkers. Unknown speech was obtained one week after the model data. Recognition accuracies of $96 \%, 87 \%$, and $79 \%$ were obtained for unknown speech durations of 10,5 , and $3 \mathrm{~s}$, respectively. The use of multiple sub-models to characterize spectral traits results in improved discrimination between speakers, particularly when short speech segments are recognized. [Work supported by U. S. Air Force, Rome Air Development Center.]

\section{2:19}

R2. A syllable parsing algorithm for telephone-quality speech. D. Kahn (Acoustics Research Department, Bell Laboratories, Murray Hill, NJ 07974)

This paper describes an algorithm for the automatic parsing of continuous speech into a string of syllables, intended for use as the "front end" of a demisyllable-based continuous speech recognizer. The algorithm takes an input an autocorrelation coefficient (ACC) parameterization of speech, an analysis independently required for our recognition process; it makes use of only the zeroth-order ACC (energy) and normalized first-order ACC. Local maxima in a severely low-pass-filtered representation of the energy signal determine potential syllabic nuclei. Some of these first-pass syllables are discarded on the basis of further tests. Compared to other syllable parsers described in the literature, this algorithm (1) was designed and tested for use with telephone speech, i.e., utterances spoken into a carbon microphone over a dialed-up phone line and bandpass filtered to $200-3200 \mathrm{~Hz}$; (2) locates potential syllabic nuclei by reference to the simple energy signal, rather than to a spectrally weighted "loudness" transformation of energy; (3) differs in many details of the true/spurious syllable decision process. Performance data for the algorithm will be presented, as will a tape recording of examples of the rather carefully spoken but truly continuous speech used to evaluate it.

\section{2:33}

R3. On the use of energy in LPC-based recognition of isolated words. $M$. K. Brown and L. R. Rabiner (Bell Laboratories, 600 Mountain Ave., Murray Hill, NJ 07974)

Recognition of isolated words by encoding speech into Linear Predictive Coefficients $(L P C)$ is well known and widely accepted as one of the better methods for speech recognition. One of the drawbacks in relying entirely on LPC for recognition, however, is that the energy information in the speech is removed during the LPC analysis. This talk discusses problems involved in combining energy pattern information with the LPC pattern information and presents results of recognition experiments with one method. The method is general and applicable to the addition of any new feature to the recognition feature set. The energy information and LPC information are combined linearly in the frame-by-frame manner utilizing the dynamic time warping (DTW) method of Itakura. The Itakura distance function, which determines the spectral difference between two frames of speech, does not lend itself to direct statistical analysis in multiple dimensions. The method for obtaining the weighting for the linear combination involves an iterative minimization of a probability of error function. The combined energy and LPC distance function was tested using a 129-word "airline" vocabulary which was designed for speaker independent, isolated word recognition. The inclusion of energy information in the recognition feature space reduces recognition error rates by an average of about $3 \%$ as compared to LPC alone.

\section{2:47}

R4. Nasal vowel identification using LPC-based formant analysis. Masahiro Hamada (Room 36-511, R.L.E., Massachusetts Institute of Technology, Cambridge, MA 02139 and Matsushita Electric Ind.)

An experiment was carried out to determine the problems associated with the identification of nasal vowels in English in the context of vowel recognition using LPC-based formant analysis. A number of tokens of three pairs of oral/nasal vowels $[\mathfrak{x}, \varepsilon, \mathrm{r}$,$] produced in similar phonetic$ contexts (such as in camp versus camp) were obtained from three male speakers. The first and second formant frequencies obtained as LPC spectral peaks from these pairs of vowels showed significant differences in distribution on the $F 1-F 2$ plane. The first formant frequencies of nasal vowels especially had apparent overlap with those of other nasal vowels, while oral vowels had no overlap with each other. The LPC spectra corresponding to the shifted formants and the pole-zero pairs introduced by the nasal cavity coupling showed substantial individual differences in shape in the first formant region, including different bandwidths and number of peaks. These facts suggest the difficulty of vowel identification in (1) using the same template for both oral and nasal vowels and (2) identifying an unknown vowel even if one knows it is nasalized.

\section{3:01}

R5. Speaker-independent discrete utterance recognition system, combining deterministic and probabalistic strategies. G. Vysotsky (Acoustics Research Department, Bell Laboratories, Murray Hill, NJ 07974)

A speaker-independent discrete utterance recognition system is described which represents utterances as sequences of segments. Each segment is labelled with one of four broad phonetic classes intended to be highly reliable over a large population of speakers. Each type of segment is parameterized by a set of acoustic features obtained from various combinations of the basic acoustic measurements provided by the front-end processor. Each sequence of segments together with its feature sets constitutes a test pattern. Each test pattern is compared with similarly constituted reference prototype patterns to obtain a recognition decision. Reference prototypes are stored in tables indexed by each possible sequence of segments. Stored in each table are means and standard deviations of segment features associated with each vocabulary word characterized by that sequence of segments. The system was evaluated using a 20 -word vocabulary which includes the ten digits. A mean recognition accuracy of about 95\% was obtained over a population of ten speakers, five of who participated in training the system. Over $90 \%$ of the errors were rejections rather than misidentifications.

\section{3:15}

R6. A suprasegmental component in a speech recognition system. J. Vaissière (Centre National d'Etudes des Télécommunications, 22301 Lannion, France)

This paper presents a method for integrating a suprasegmental component into an automatic speech recognition system. It aims to narrow down the number of word candidates proposed by the lexical component by rejecting candidates whose fundamental frequency $(F 0)$, durational, and intensity patterns do not match with the observed prosodic pattern of an input word. A stylized $F 0$ pattern for each word in the lexicon is described by a network specifying $F 0$ rises, levels, and falls. Expected durational relationship as which of two arbitrary segments (i.e., phonemes and/or syllables) within the word is longer are specified by a matrix: these dichotomous relations are marked in the matrix only when they are invariant, regardless of the context, i.e., the position of the word in the utterance, and of speakers. For intensity, a similar matrix is constructed for each word. As expected, the degree of suprasegmental constrains increases as the length of the words become longer. These suprasegmental characteristics are determined, manually or automatically, by using word-independent general rules, or by specific rules which can apply to a subset of the lexical words related to the task-dependent syntax [which constrains the position( $\mathbf{s})$ of a word in the utterance, and consequently increases prosodic expectations for that word].

\section{3:29}

R7. Speaker independent isolated digit recognition using hidden Markov models. S. E. Levinson, L. R. Rabiner, and M. M. Sondhi (Acoustics Research Department, Bell Laboratories, Murray Hill, NJ 07974)

A method for speaker independent isolated digit recognition based on modeling entire words as discrete probabilistic functions of a Markov 
process is described. Training is a three-part process comprising conventional methods of linear prediction analysis and vector quantization of the LPCs followed by an algorithm [L. E. Baum, Inequalities 3, 1-8 (1972)] for estimating the parameters of a hidden Markov process. Recognition utilizes linear prediction and vector quantization steps prior to maximum likelihood classification based on the Viterbi algorithm [A. J. Viterbi, IEEE Trans. Inf. Theo. IT-13, 260-269 (1967)]. After training based on a 1000-token set, recognition experiments were conducted on a separate 1000 -token test set obtained from 100 new talkers. In this test a $3.5 \%$ error rate was observed which is comparable to that measured in an identical test of an LPC/DTW system [L. R. Rabiner et al., IEEE Trans. Acoust. Speech Signal Process. ASSP-37, 336-349 (1979)]. The computational demand for recognition under the new system is reduced by a factor of approximately ten in both time and memory compared to that of the LPC/DTW system. Issues of selection of model structures, averaging techniques to obtain model stability, and methods of compensating for finite training set size are also discussed.

\section{3:43}

R8. On the effects of varying filter bank parameters on isolated word recognition. B. A. Dautrich, L. R. Rabiner, and T. B. Martin (Acoustics Research Department, Bell Laboratories, Murray Hill, NJ 07974)

The vast majority of commercially available isolated word recognizers use a filter bank analysis as the front end processing for recognition. It is not well understood how the parameters of different filter banks (e.g., number of filters, types of filters, filter spacing, etc.) affect recognizer performance. In this talk we present results of performance evaluation of several types of filter bank analyzer in a speaker trained, isolated word recognition test using dialed-up telephone line recordings. We have studied both DFT (discrete Fourier transform) and direct form implementations of the filter banks. We have also considered uniform and nonuniform filter spacings. The results indicate that the best performance (highest word accuracy) is obtained by both a 15-channel uniform filter bank and a 13-channel nonuniform filter bank (with channels spaced along a critical band scale). In comparison to a conventional linear predictive coding (LPC) word recognizer, the performance of the best filter bank recognizers was, on average, several percent worse than that of an eighth order LPC-based recognizer.

R9. Some experiments of a speaker-independent isolated word recognition using the Selective Weighted Matching. $K$. Mizuno, H. Iizuka, and K. Yamada (Oki Electric Industry Co., Ltd., 550-5 Higashi-asakawa-cho, Hachioji-shi, Tokyo 193, Japan)

At the last meeting in Chicago, we reported the speaker-independent isolated word recognition system using the Selective Weighted Matching. This paper refers to the results of some experiments to investigate the relation between recognition accuracy and following items: (1) the number of training talkers, (2) the number of reference patterns, and (3) the recognition accuracy for 100 test talkers. In these experiments, we used a vocabulary (digits and two command words in Japanese) uttered over a telephone line by 500 talkers. Concerning (1), we made recognition tests for 100 test talkers within 96 reference patterns. The number of training talkers was varied from 100 to 400 . In the case of 100 talkers, the recognition accuracy was $96.96 \%$ for the training talkers and $93.37 \%$ for the test talkers. In the case of 400 talkers, the results were $95.37 \%$ and $94.17 \%$, respectively. By increasing the training talkers, the recognition accuracy was inclined to decrease for the training talkers and to increase for the test talkers, and the difference between them was diminished. From the results of these experiments, we decided the practical conditions for our recognition system.

\section{4:11}

R10. A nonuniform sampling approach to isolated word recognition. F. Soong (Acoustics Research Department, Bell Laboratories, Murray Hill, NJ 07974)

Speech signals are known to consist of both stationary and transient parts. The transient part is invariably shorter than it stationary counter- part. The standard uniform sampling approach for creating word reference patterns for speech recognition will naturally emphasize the longer steady-state portions more than the short transitional portions. Since the transient portions of words contain valuable consonantal information for recognition, a nonuniform sampling approach seems to be a natural choice for potentially improving the recognizer performance as well as for reducing the overall sampling rate and computational complexity of the recognizer. However, there are some inherent difficulties in this approach. They are (1) artifacts and distortions such as breath noise, mouth clicks, etc. contribute non-negligible spectral variations and result in oversampling in these regions; (2) the inherent variance of the spectral estimate of the transitional regions is much larger than than of a steady-state region. Nonuniform sampling therefore suffers high uncertainty in its spectral estimate since the high variance regions are sampled more often. Recognition results are presented on a 39-word, alpha-digit vocabulary and compared with those from a uniform sampling approach.

4:25

R11. Speaker-independent recognition of English letters. Ronald A. Cole, Michael S. Phillips, Scott M. Brill, Philippe Specker, and Andrew P. Pilant (Carnegie-Mellon University, Department of Computer Science, Pittsburgh, PA 15213)

An isolated word recognition system is described which classifies English letters using features extracted from speech. Speech was sampled at $16000 \mathrm{~Hz}$, and pitch, endpoint detection, and location of vowel onset and offset were computed directly from the digitized data. A 128-point DFT was performed every $3 \mathrm{~ms}$ using a 20-ms window, and frequency samples were linearly compressed to 54 coefficients spanning the frequency range from 62.5 to $7000 \mathrm{~Hz}$. Most feature extraction algorithms used this 54 coefficient representation. A knowledge-engineering approach was used to create feature extraction algorithms from digital spectrograms of 2080 tokens of English letters produced by ten male and ten female talkers. Featural measurements included voice onset time, onset abruptness, formant frequencies, and formant trajectories, and all made use of the end: points of the sound and/or vowel onset as temporal anchors. Feature values from a subset of the 2080 utterances were used to train the classifier, and system performance was evaluated on the basis of errors in classifying the remaining utterances. Features will be described and recognition results will be reported for each decision point within the system. [Supported by NSF.]

\section{4:39}

R12. Tuning to the speaker: dynamic adaptation of statistical parameters in isolated letter recognition. Richard M. Stern and Moshe J. Lasry (Department of Electrical Engineering, Carnegie-Mellon University, Pittsburgh, PA 15213)

The performance of a feature-based, speaker-independent recognition system can be improved by enabling the system to learn the acoustical characteristics of individual speakers. Even when features are designed to be speaker-independent, it is typically observed that for a given feature and pair of letters within-speaker variation can be less than betweenspeaker variation. For example, across all speakers a given feature may have an expected value of 5 for the letter $M$ and 10 for $N$, but a certain speaker may produce average values of 9 for $M$ and 14 for $N$. In such cases it is necessary to adjust statistical feature parameters to the individual speaker to obtain optimal recognition performance. This paper describes a dynamic adaptation procedure for updating expected feature values during recognition. The algorithm uses maximum a posteriori probability (MAP) estimation techniques to update the mean vectors of sets of measure values on a speaker-by-speaker basis. In updating these mean vectors, the algorithm makes use of the observations input thus far, the relative variability of the features' means within and across subjects, and the covariance of the mean vectors within and across the various letters or sets of letters. The use of tuning produced a dramatic decrease in error rates for certain speakers and letters. [Work supported by NSF.] 
heuristics involved. Psychological evidence suggests that prosodic and robust segmental features may be used as preliminary decision criteria in human speech perception. In this paper we present attempts to apply such heuristics to the design of VLVR systems. As a data base a 20000 word vocabulary has been compiled providing phonemic, prosodic, and pragmatic information. Based on this corpus, the tradeoffs between the robustness of certain features and their power to reduce the search space have been studied. Our results indicate that combining prosodic information (syllable counts, stress patterns) with a set of robustly detectable features (frication, stops, vowel nuclei of stressed syllable) can reduce the vocabulary size to groups of less than $\mathbf{4 0 0}$ words. Additional potentially useful prosodic features, e.g., rhythmic patterns, are currently being investigated. the recognition system the test utterance was first analyzed using Fisher linear discriminant functions, with threshold weights individually set for each pairwise decision in order to minimize misclassifications. When a decision could not be made with certainty, classification was performed using a maximum likelihood procedure assuming multivariate Gaussian statistics. The sequential use of nonparametric and parametric discriminant functions produced superior classification to that obtained with either of the separate analyses. The overall system structure will be discussed in terms of practical tradeoffs between the number of features used at each decision node and the system's overall probability of error. [Work supported by NSF.]

\section{5:07}

R14. A powerful post-processing algorithm for time domain pitch trackers. Philippe Specker (Department of Computer Science, CarnegieMellon University, Schenley Park, Pittsburgh, PA 15213)

Existing pitch tracking algorithms are not precise nor reliable enough to be useful in feature-based recognition systems. It is possible, however, to analyze the errors produced by a particular algorithm and then reduce these errors using post-processing techniques. Error patterns were analyzed for a time-domain pitch tracker [W. H. Tucker and R. H. T. Bates, IEEE Trans. ASSP-26, 597-604 (1978)] and a post-processing algorithm, based on artificial intelligence techniques, was written in order to eliminate errors. Performance was compared for the original and modified versions of the pitch tracker for a number of speakers using both isolated words and sentences. All types of errors were reduced by the post-processing algorithm. Voiced-voiceless decisions were performed with less than $1 \%$ error for 2080 letters produced by males and females. The fundamental frequency microstructure was tracked sufficiently well to be used in extracting phonetic features in a feature-based recognition system.

\section{$5: 21$}

R15. Very large vocabulary recognition (VLVR): using prosodic and spectral filters. Alex Waibel (Computer Science Department, CarnegieMellon Uiversity, Pittsburgh, PA 15213)

The use of Very Large Vocabularies ( 20000 words) imposes two major constraints on the design of isolated or connected word recognition systems: the efficient reduction of the large search space to a subvocabulary of manageable size [V. Zue and D. Shipman, J. Acoust. Soc. Am. Suppl. 1 71, C7 (1982)] and the robustness of the search space reduction

\section{$5: 49$}

R17. Unifying dynamic programming methods. James K. Baker (DRAGON Systems, Inc., 173 Highland Street, West Newton, MA 02165)

Dynamic programming has come to be widely used and accepted in automatic speech recognition. However, two different but similar applications have often been described more in terms of their differences than their similarities. On the one hand, dynamic programming is used to find the best nonlinear dynamic time warping to align two instances of a word. On the other hand, dynamic programming may be used to find the best state sequence for a hidden Markov process. Not only are these procedures essentially equivalent, but significant generalization comes from an explicit unification. Dynamic programming may be used not only to align two instances of a word, but also to align an instance of a word with an arbitrary finite state model for the word, or even to align two arbitray models. Multiple instances of a word may contribute to a single model, and multiple passes on a finite set of training data can be used to further refine word models. 


\title{
Session S. Speech Communication III: Perception in Children and Hearing Impaired
}

\author{
Robert J. Porter, Chairman
}

Kresge Hearing Research, Laboratory of the South, New Orleans, Louisiana 70122

Chairman's Introduction-2:00

\section{Contributed Papers}

2:05

S1. Identification of $\mathrm{CV}$ syllables by children and adults when task demands are varied. Lois L. Elliott and Lu Ann Clifton (Northwestern University, 2299 Sheridan Road, Evanston, IL 60201)

We previously reported that normal children require higher intensities, than adults, to identify three CV syllables [ba,da,ga] [Elliott et al., Percept. Psychophys. 30, 411-416 (1981)]. We replicated the previous experimental condition and, also, presented a 5-CV condition (the $3 \mathrm{CVs}$ used previously plus $2 \mathrm{CV}$ located at the category boundaries). Intensity ranges for each 6-year-old and adult $S$ assured performance that varied from less than $50 \%$ to better than $90 \%$ correct. Thus all Ss received approximately equal numbers of positively reinforced trials. Since responses were limited to "ba," "da," and "ga," Ss could never be correct when responding to the two extra CVs of the 5-CV test condition. It was hypothesized that if 6-year-olds were less attentive to the task than adults, they would perform relatively more poorly on the 5-CV task than the 3 $\mathrm{CV}$ task, when compared to adults, because of the longer test run and because of the lower overall level of positive reinforcement. An ANOVA showed no differences in "identification thresholds" or slopes of the identification functions between the 3- and 5-CV conditions, for either children or adults. This provides additional support for the conclusion that the higher intensities required by normal children do not reflect lack of attention. [Supported, in part, by NSF.]

\section{2:20}

S2. Perceptual test of the error severity model of the recovery process for articulatory disorders in children. Donald J. Sharf (Speech and Hearing Sciences Program, University of Michigan, 1111 Catherine Street, Ann Arbor, MI 48109) and Ralph N. Ohde (Bill Wilkerson Hearing and Speech Center and Vanderbilt University School of Medicine, 1114 19th Avenue South, Nashville, TN 37212)

According to the error severity model of the recovery process from articulatory disorders, children progress from errors of omission to substitution to distortion before correct sound production. Inherent in this model is the assumption that the distorted phoneme is perceived as a unique sound category. Although categorical perception studies have shown within category perception, by far the most robust effects have been observed across categories. Consequently, categorical perception theory would provide only weak support for the above assumption. Since the perception of a distorted phoneme category is fundamental to the error severity model, this study tested the perceptual reality of the distorted $/ r /$. In order to determine if subjects could categorize acoustic properties of /Cej/-type utterances varying in $F 2$ and $F 3$ onset frequencies as distorted $/ r /$, speech pathologists identified two 25 -stimuli manifolds synthesized so as to approximate $/ \mathrm{r} /, / \mathrm{w} /$ and defective $/ \mathrm{r} /$ sounds modeled after child and adult vocal tracts. Subjects scaled the stimuli between $/ r /$ and $/ w /$ in one condition and labeled them as $/ r /, / w /$, or distorted $/ r /$ in another condition. The results showed that subjects consistently identified a distorted $/ \mathrm{r} /$ category for the adult manifold but not for the child manifold. These results cast some doubt that the distorted $/ r /$ is a viable perceptual category for speakers who hold the greatest interest among speech pathologists-children. [Work supported in part by NINCDS.]

\section{2:35}

S3. Dichotic listening and stuttering severity: statistical significance or sampling error? Gordon W. Blood and Ingrid M. Blood(Department of Communication and Theatre, Miami University, Oxford, $\mathrm{OH}$ 45056)

The clinical importance of dichotic listening tasks (DLT) in stuttering should be established. The purpose of the present series of experiments was to determine the relationship between stuttering severity and dichotic ear preference scores. Experiment I, conducted in 1978, administered a DLT to 51 stutterers between the ages of 7 and 15 years. Results revealed that the right ear preference (REP) weakened as the severity of the stuttering decreased. Experiment II, conducted in 1980, involved 24 stutters between the ages of 8 and 13 years. Analyses revealed that the REP weakened as the severity of stuttering increased. Experiment III, conducted in 1982, with a sample of nine stutterers (ages 9-11 years) confirmed the results of experiment II. Results of different analyses $\left(X^{2}\right.$, phi coefficient, $t$ test) for significance of ear preference and analysis of the data as one cumulative study or three independent studies will be presented. Discussed will involve the limitations of future research with DLT's stuttering and its usefulness as a clinical tool in light of these findings.

\section{2:50}

S4. Speech perception in noise as a function of hearing status. $M$. L. Matthies, R. C. Bilger, and M. Yuchtman (Department of Speech and Hearing Science, $901 \mathrm{~S}$. Sixth Street, University of Illinois, Champaign, IL 61820)

Speech-recognition scores were obtained for two recorded tests (SPIN and MRT) at signal-to-babble ratios ranging from +7 to $-1 \mathrm{~dB} \mathrm{~S} / \mathrm{B}$ for 90 subjects. These subjects were selected to meet criteria of age, audiometric configuration, and hearing-aid use which defined five subgroups of 18 subjects each (college age, normal hearing; adult, normal hearing; adult, high-frequency hearing loss; adult, successful hearing-aid use; adult, unsuccessful hearing-aid use.) To assess the effects of increasing babble level on speech recognition scores, one should first try to minimize the group differences. We attempted to equate the three hearing-impaired groups with the normal subjects in three ways, equating the groups for the mean at the optimal S/B, across all S/B's and by graphically shifting that data to the normal's S/B function. The graphical method did not preserve the shape of the S/B function but the adjusted means made it possible to look primarily at the noise effects. Conclusions based on the corrected data about the speech recognition performance of hearing-impaired subjects in noise are substantially different from those based on the uncorrected data. In the corrected data, there is little difference between the hearing-impaired and the normal-hearing groups on the MRT and the apparent affects of S/B on the SPIN scores are substantially reduced. [Work supported under contract to NINCDS.]

\section{3:05}

S5. Frequency response considerations in the acoustic coupling of hearing aids and telephones. Richard G. Stoker, Marilyn French-St. George, and Alice E. Holmes (Speech and Hearing Clinic, The Pennsylvania State University, 110 Moore Building, University Park, PA 16802)

Four types of signal processing (high-frequency emphasis, low-frequency emphasis, bimodal emphasis centered at $900 \mathrm{~Hz}$, and flat gain) 
were evaluated for their effects upon the perception of speech over the telephone when used with a hearing aid microphone. The telephone receiver and hearing aid microphone were acoustically coupled and recordings made using a dummy head. These recordings were later presented to three subject groups (normally hearing, moderately impaired, and severely impaired). The data suggest that the frequency response of conventional hearing aids may be inappropriate for the acoustic coupling of hearing aids and telephone receivers, especially in backgrounds of noise. Additionally, it was demonstrated that subjects do not reliably select the test conditions which give them maximum objective scores when given the opportunity to personally manipulate frequency response and signal level.

\section{$3: 20$}

S6. Telephone listening ability in hearing impaired subjects. Alice E. Holmes (Department of Speech, University of Florida, Gainesville, FL 32611) and Thomas Frank (Department of Communication Disorders, The Pennsylvania State University, State College, PA 16801)

The purpose of this study was to investigate telephone listening ability of the hearing impaired. Forty-five subjects with bilateral sensorineural hearing losses were divided into three groups ( $N=15 /$ group) based on the audiometric configuration of their test ear. The subjects were evaluated using conventional and alternative telephone listening strategies by assessing their discriminations of word lists presented at 86-dB SPL (simulating the output of a standard telephone handset) and at their Most Comfortable Loudness (simulating the output of a telephone amplifier handset). The results indicated that subjects with precipitous drop hearing losses had similar word discriminations across the listening modes which included a TDH-39 earphone, unaided telephone, and their hearing aids acoustically coupled to a telephone handset and at both 86-dB SPL and their Most Comfortable Loudness (MCL). Subjects with gradual slope or flat hearing losses had similar word discriminations across the listening modes at either 86-dB SPL or MCL. However, these subjects with gradual slope and flat hearing losses had better word discriminations in the listening modes when the word lists were presented at MCL compared with 86 dB SPL.

\section{$3: 35$}

S7. The feasibility of deriving an articulation index based scheme for assessing residual auditory function in listeners with sensorineural hearing impairment. Chaslav V. Pavlovich (Department of Communicative Disorders, University of Mississippi, University, MS 38677)

In the first experiment the AI theory was applied to audiograms of normal and sensorineural hearing impaired individuals in order to predict speech discrimination under various conditions. Good predictions were found for the normal and the lesser impaired subjects, but not for those with greater impairments. Also, poor correlation was obtained between the reduction in the $A I$ for everyday speech and different subjective estimates of auditory handicap. In the second experiment it was investigated whether the AI predictions for presbyacusic individuals could be improved by making use of the frequency unspecific proficiency factor concept as suggested by Fletcher [J. Acoust. Soc. Am. 24, 490 (1952)] and Dugal et al. [in Acoustical Factors Affecting Hearing Aid Performance, edited by G. A. Studebaker and I. Hochberg, (University Park Press, Baltimore, 1978), Chap. 17]. The results indicate that the proficiency factor is not frequency independent. It assumes the value of 1 at those frequencies where hearing sensitivity is normal and it is substantially reduced for frequencies where hearing threshold is elevated.

\section{3:50}

S8. Recognition of selected French vowels and consonants by deaf children: lipreading and coarticulation. Michèle Gentil (IBM-France, Centre Scientifique, 36 Avenue Raymond Poincaré, 75116 Paris, France)

The visual recognition of selected French vowels and consonants by deaf children was studied. Thirteen vowels were associated with [1] in the final position and/or placed between [p] and [1]. Sixteen consonants were combined with the extreme vowels on the vocalic triangle [i-a-u] in nonsense words of consonant-vowel type. Two speakers were chosen for this investigation. This research determined confusion matrices. Concerning vowels, the extreme vowels on the vocalic triangle were the most easily recognized visually. Concerning consonants, the results of visual confusions revealed four basic homophenous categories or viseme groups. Three of these categories were based on lip movement $[p-b-m][f-v][s-3]$. The fourth category consisted of consonants produced inside the mouth $[\mathrm{t}-\mathrm{d} \cdot \mathrm{n}-\mathrm{s}-\mathrm{z}-\mathrm{k}-\mathrm{g}-\mathrm{n}-\mathrm{R}]$. Besides in this study emphasis was placed on coarticulatory effects which make lipreading difficult. The difficulty of recognizing depended on the vocalic environment: most difficulty when followed by [u], intermediately difficult when followed by [i], least difficult when followed by [a]. Inter-speaker variability was evident.

\section{4:05}

S9. Development changes in perceived stimulus structure in consonantvowel and vowel stimuli. Lynne E. Bernstein, David Talkin, Rosemary Condino, and Rachel E. Stark (Communication Sciences Research Laboratory, John F. Kennedy Institute, Baltimore, MD 21205)

It was hypothesized that developmental changes take place in the ability to extract acoustic-phonetic structure from the speech signal. In order to demonstrate developmental change, Garner's two-choice, speededclassification paradigm was adapted for use with children [Am. Psych. 25, $350-358(1970)]$. In this paradigm, two stimulus dimensions are presented under control, correlated, and orthogonal experimental conditions. Patterns of results across conditions are interpreted as indicative of certain perceptual structures, viz., integral versus separable processing. Stimulus dimensions being tested with adults and eight-year-olds are (1) consonant identity (/ba/ versus /da/) and pitch (125 vs $\left.165 \mathrm{~Hz} F_{0}\right)$ [cf. Wood, Percept. Psychophys. 15, 501-508 (1974)]; and (2) vowel identity (/a/ versus / i/ and pitch (125 vs $165 \mathrm{~Hz} F_{0}$ ). It was hypothesized that adults process these two stimulus sets in terms of, respectively, integral and separable structures, but that children process both sets in terms of similarity struc ture (integrally). Preliminary results confirm predictions for children but suggest that adults process pitch integrally for both stimulus sets. Implications from a larger group of subjects will be discussed in terms of a theory of speech perceptual development. [Work supported by $\mathrm{MCH}$ Training Grant Project \#917 and March of Dimes Grant \# 12-84.]

\section{$4: 20$}

S10. Emergence of vocants in infant utterances. R. E. Stark, D. Talkin, J. M. Heinz, and J. Bond (Communication Sciences Research Laboratory, John F. Kennedy Institute, Baltimore, MD 21205)

This preliminary study was designed to determine if the point vowels $/ \mathrm{a} /, \mathrm{i} / \mathrm{and} / \mathrm{u} /$ emerge in a particular order and within specific age ranges in the productions of infants. Vowel-like sounds (vocants) were selected randomly within certain general criteria from the cooing, expansion, and babbling periods of vocal development in two normal female infants. The formant frequencies of these vocants were estimated, at several points, by inspecting the wideband spectrogram, a 32-ms spectral section, an inverse LPC spectrum, and the frequencies and bandwidths obtained by finding the roots of the denominator polynomial of the LPC model. The order of the LPC model was chosen adaptively to provide frequency resolution consistent with the fundamental frequency of voicing for each frame in order to minimize the interaction between harmonic and resonance locations. The results indicated (1) only modest changes in formant structure from the cooing to the expansion period and (2) a frequent lowering of the first formant and raising of the second in the babbling period, suggesting that an $/ \mathrm{i} /$-like vocant does not emerge until the babbling period. Perceptual data for these vocants will be reported. [Work supported by NICHD grant HD11970.]

\section{$4: 35$}

S11. Effects of mild hearing loss and age on speech recognition in noise. Judy R. Dubno, Donald D. Dirks, and Donald E. Morgan (Division of Head/Neck Surgery, UCLA School of Medicine, Los Angeles, CA 90024)

Previous investigations have established that, under identical test conditions, hearing-impaired listeners have more difficulty than normal- 
hearing listeners understanding speech in noise. Using an adaptive strategy, the effect of mild sensorineural hearing loss on speech recognition in babble was evaluated and the extent of the interaction of mild hearing loss with adult listeners' chronological age was examined. The signal-to-babble ratio required to achieve $50 \%$ recognition was measured for three speech materials presented at 56, 72, and $88 \mathrm{~dB}$ SPL; likewise, performance without babble was measured. Four sets of subjects $(N=18$, each set) were tested: normal-hearing listeners $<44$ and $>65$ years of age, and hearing-impaired subjects $<44$ and $>65$ years. Subjects were selected so that age-group audiograms were closely matched. For results in babble, a consistent and statistically significant difference was demonstrated between the groups differentiated by age and by hearing loss, while only the latter effect was present in the absence of babble. Significant differences in articulation indices (AIs) between the two age groups were found for results in babble only, while no differences in AIs were seen between the two hearing loss groups under either listening condition. [Work supported by NINCDS.]

\title{
TUESDAY AFTERNOON, 9 NOVEMBER 1982
}

BROWARD AND PALM BEACH ROOMS, 2:00 TO 5:05 P.M.

\section{Session T. Underwater Acoustics III: Scattering and Noise}

\author{
Harlan G. Frey, Chairman \\ Applied Research Laboratories, University of Texas, P. O. Box 8029, Austin, Texas 78712
}

Chairman's Introduction-2:00

\section{Contributed Papers}

\section{2:05}

T1. Backscattering of sound in shallow water. T. G. Muir, L. A. Thompson, J. A. Shooter, and T. E. DeMary (Applied Research Laboratories, The University of Texas at Austin, Austin, TX 78712

Measurements are reported on the backscattering of sound from an ocean surface and sand bottom overlying the continental shelf near San Diego, California. Data were acquired in the $1-25 \mathrm{kHz}$ band with a directive parametric source at a grazing angle of $10^{\circ}$. The bottom was a $\sim 5-\mathrm{m}$ layer of coarse grained sand and clay (mean grain size $0.57 \mathrm{~mm}$ ) that contained many shell fragments and sublayers. The bottom backscattering coefficient was found to be $-40 \mathrm{~dB} r e: 1 \mathrm{~m}^{2}$, independent of frequency. Surface backscattering was measured in a shoaling sea containing biologic scatterers. The surface heights were Gaussian distributed (rms wave height $=0.35 \mathrm{~m}$ ) and had a power spectral density that peaked near 80 $\mathrm{MHz}$ and decayed as the fifth power of frequency. The surface backscattering coefficient was found to be $-43 \mathrm{~dB} r e: 1 \mathrm{~m}^{2}$, with no frequency dependence. Statistical data in the form of probability distributions (peak acoustic amplitudes in 500 sample ensembles at each frequency) show a Rayleigh distribution for bottom backscattering and high-frequency (10$25 \mathrm{kHz}$ ) surface backscattering. Low-frequency surface backscattering ( $1-5 \mathrm{kHz}$ ) departs from the Rayleigh form due to the prevalence of highamplitude echoes from fish. [Work supported by the Office of Naval Research.]

\section{2:20}

T2. Horizontal and vertical covariance of surface reverberation. Gary R. Wilson (Applied Research Laboratories, the University of Texas at Austin, P. O. Box 8029, Austin, TX 78712)

Lake surface reverberation was measured simultaneously on four horizontally separated and nine vertically separated staves in two linear arrays. The vertical array was found to maintain a significant level of coherence at much larger separations than the horizontal array. The normalized horizontal covariance was constant with time, while the normalized vertical covariance oscillated slowly with a period that depended on the separation of the vertical staves. The measurements were compared to a theoretical model of reverberation by $D$. Middleton. The model was implemented with a uniform planar distribution of surface scatterers and adequately predicted the horizontal spatial covariance and the time dependence of both the horizontal and vertical covariance. However the simple planar surface model was unable to model the vertical spatial covariance, indicating a need for a more detailed description of the surface.

\section{2:35}

T3. Backscatter from the ocean surface at low frequency. James E. Barger (Bolt Beranek and Newman Inc., Cambridge, MA 02138)

Backscatter strengths from the ocean surface were measured using a vertically directive, impulsive sound source having a $26-\mathrm{Hz}$ bandwidth centered at a frequency of $52 \mathrm{~Hz}$. The ocean surface was characterized by a 6.3-m swell having an $80-\mathrm{m}$ wavelength, with $15-\mathrm{kt}$ wind waves superposed. Backscatter strengths were measured as functions of the vertical grazing angle $\theta$ and of the horizontal angle $\psi$ between the swell and the backscatter directions. The results are summarized by the expression for scattering strength, SS $=-66.5+53 \sin \theta+8.7 \cos \psi$. These values of scattering strength are somewhat larger than those reported by Brown and Saenger [M. V. Brown and R. A. Saenger, J. Acoust. Soc. Am. 52, 944-960 (1973)], but their data were not obtained from surfaces having such a large swell. The backscattered intensities have swell-induced amplitude modulation, suggesting that backscatter from wind waves is periodically shadowed by swell troughs. [Work supported by DARPA.]

\section{2:50}

T4. Multiple scattering with applications to fish-echo processing. $T$. K. Stanton (Marine Studies Center, University of Wisconsin, Madison, WI 53706)

A general expression has been derived and evaluated for the backscattered echo energy of an acoustic pulse due to a collection of identical randomly distributed isot ropic scatterers. Excess attenuation of the signal due to the extinction cross section of the scatterers as well as second-order scattering have been taken into account. Special attention is focused toward the numerical evaluation of second-order scattering effects. The expression is evaluated for three scattering geometries. It is shown in each geometry that when the absorption cross section of the scatterers is small, second-order scattering at least partially offsets effects due to excess attenuation in the low-to-moderate attenuation region. It is shown that second-order scattering can be important in fish-echo processing where the echo energy is quite often analyzed. The directional characteristics, acoustic frequency of the pulse in relation to the resonance frequency of the swimbladder (if any), and the degree of randomness of the spatial 
distribution of the fish determine the degree to which second-order scattering plays a role in this area. [Work supported by Office of Naval Research N00014-80-C-0667.]

\section{3:05}

T5. Con- and de-convolution of the fish scattering PDF and echo PDF for single transducer sonar. C. S. Clay (Department of Geology and Geophysics, University of Wisconsin, Madison, WI 53706)

This paper is a continuation of our studies of acoustic methods to measure fish abundance. The integral equation for the probability density function (PDF) of fish echoes is in Clay and Medwin [Acoustical Oceanography, pp. 476-482 (1977)]. It relates the PDF of the output of the sonar $w_{E}(e)$ to the PDF of the sonar for a target at random location in the beam $w_{1}(b)$, and the PDF of the scattering process at the fish $w_{R}(e)$, where $e$ is echo amplitude and $b$ is the transducer response. Our procedure is to adjust parameters in the two-parameter Rice PDF (the amplitude of the envelope of a sine wave plus noise) until $w_{E}(e)$ matches experimental data. Numerical computations are somewhat tedious. In this paper I use nonlinear transformations $b=\exp (x)$ and $e=e_{0} \exp (y)$ to change the integral to a standard form of the convolution integral. One can do a Fourier transformation of the result and then express the convolution as the product of $z^{--}$transforms. Computations with the $z^{--}$transforms are simple and may become the basis of ways to determine $w_{F}(e)$ directly. [Work supported by Office of Naval Research N00014-80-C-0667.]

\section{$3: 20$}

T6. Prediction of ocean basin bottom reverberation using a rangedependent reverberation model. K. R. Nicolas, E. R. Franchi, and A. Tolstoy (Code 5160, Naval Research Laboratory, Washington, DC 20375)

The NRL model for underwater acoustic reverberation incorporates range dependent bathymetry and sound speed profiles. An extended ray theoretic approach for propagation estimates is combined with boundary scattering models to predict, among other measures, reverberation time histories. This paper presents model predictions of ocean basin and margin bottom reverberation for a monostatic source-receiver geometry at a deep water site in the Northeast Pacific. The reverberation time histories are calculated for a number of radials which are selected to include prominent features such as seamounts, fracture zones, islands, and continental slopes. These time histories are azimuthally convolved with a receiver beam pattern to approximate the total basin reverberation response. The strong backscattered returns from both the continental shelf of North America and large fracture zones are of particular interest. Their strength can be 30 to $50 \mathrm{~dB}$ above the normal reverberation decay levels, making them a serious interference, even when located in the beam sidelobes. These predictions provide valuable information for source-receiver design, signal selection, and pulse repetition rates to be used in future deep sea experiments. [Work supported by Naval Electronic Systems Command, Code 612.]

\section{T7. Abstract withdrawn.}

$$
\text { 3:50 }
$$

T8. An acoustic bottom microprofiler and its application to highfrequency acoustic scattering. Y. Igarashi and R. L. Allman (Naval Ocean Systems Center, San Diego, CA 92152)

Small-scale bottom roughness data are required for evaluation of high-frequency scattering models. A shipboard-launched acoustic microprofiler has been designed for obtaining detailed traces of bottom contours over 6-ft horizontal line scans in random orientations. The system consists of a $5.75-\mathrm{MHz}$, downward-looking transducer and electronic instrumentation package mounted on a cable-lowered frame, which is set on the sea floor. A roughness scale of approximately $1 \mathrm{~cm}-1 \mathrm{~m}$ is measured. Estimated vertical and horizontal resolutions are 1 and $8 \mathrm{~mm}$, respectively. Measurements were made in two bottom areas that are nominally flat but have contrasting small-scale features. Computed roughness height power spectral density functions showed that the magnitude of the spectral functions differed by about $15 \mathrm{~dB}$, but the slopes of the spectra both exhibited a power-law decay of approximately 2.50 . These results are directly applicable to many theoretical scattering formulations with regard to the magnitude and frequency dependence of scattering functions. [Work supported by the Naval Sea Systems Command.]

\section{4:05}

\section{T9. Abstract withdrawn.}

\section{$4: 20$}

T10. Sound-channel effects on ambient noise spectra. Orest Diachok and Roger Gauss (Naval Research Laboratory, Washington, DC 20375)

Possible causes of the observed site dependence of deep ocean noise spectra below $100 \mathrm{~Hz}$ are examined through a coherent ambient noise prediction scheme due to Ingentio and Kuperman. The model accounts for propagation from a random distribution of surface dipoles in a rangeindependent, compressional sound-velocity structure; hence data-theory comparisons were limited to deep ocean abyssal plain environments. A variety of environments from open ocean (with varying wind speeds) to noisy ice-edge configurations were considered. Results below $100 \mathrm{~Hz}$ reveal a frequency-dependent, sound-channel-controlled amplification of the noise-source spectra which is sensitive to bottom sound-speed profile/ attenuation coefficient and sea-surface scattering loss. Below $5 \mathrm{~Hz}$ the computed amplification accurately accounts for the apparent discrepancy between theoretical and measured noise due to nonlinear interaction of surface waves. Above about $50 \mathrm{~Hz}$ the computed noise-source spectral shapes in the open ocean and at the ice edge were nearly identical, suggesting that the same mechanism (possibly subsurface bubble cavitation) is responsible. Estimated sound-channel effects on the frequency and windspeed dependence of the noise spectra between 5 and $50 \mathrm{~Hz}$, where a different mechanism(s) predominates, will also be presented. [Work supported by 425AR, NAVELEX 612.]

\subsection{5}

T11. Modeling the vertical directionality of ambient noise in a range dependent environment using the adiabatic invariant approximation method. John Northrop (Naval Ocean Systems Center, Code 5311, San Diego, CA 92152)

The adiabatic invariant approximation (AIA) method of tracing sound rays in a range dependent environment was used in computing the vertical arrival angle of ambient noise at various frequencies and depths. Both surface- and bottom-reflection coefficients were incorporated in the model so that calculations could be performed out to any number of convergence zones. Noise from shipping and storms were treated as monopoles and dipoles, respectively. It is shown how the results can be used to choose the best operating depth for a vertical line array.

\section{4:50}

T12. A linear regression model for the wavenumber-frequency spectrum of the turbulent boundary layer pressure fluctuations. Y. F. Hwang (David Taylor Naval Ship R\&D Center, Bethesda, MD 20084)

The rationale of using a linear regression model for estimating the wavenumber-frequency spectrum of the turbulent boundary layer pressure fluctuations is presented. The contention is that the wavenumberfrequency spectra, normalized by the convected ridge value at the respective frequency, are the same for all frequencies if the wavenumber is expressed in terms of a nondimensional wavenumber. The nondimensional wavenumber is the streamwise wavenumber that is normalized by the convected wavenumber at the respective frequency. The normalized wavenumber spectrum is then approximately represented by a set of discrete spectral coefficients. The linear regression model relates the response of a measuring system to these discrete spectral coefficients. The spectral coefficients are determined by least-square minimization of a set of randomly collected response data. 


\title{
Session U. Underwater Acoustics IV: Space-Time Signal Processing (Précis-Poster Session)
}

\author{
Richard R. Rojas, Chairman
}

Naval Research Laboratory, Code 5000, Washington, DC 20375

\author{
Chairman's Introduction-8:00
}

\section{Contributed Papers.}

\section{8:05}

U1. Deterministic signal processing of acoustic array outputs. Philip L. Stocklin (Consulting Physicist, 25 Ledgewood Court, Norwich, CT 06360)

Acoustic signal processing currently relies heavily upon probabilistic assumptions concerning data and upon statistical properties of data to develop efficient processing principles. In contrast, deterministic signal processing uses only the actual sensor output amplitudes over a finite time (a data set) to estimate signal waveform and input power for that data set, employing no probabilistic assumptions or statistically based techniques. The major result presented is a demonstration of the improvement in output $\mathbf{S} / \mathrm{N}$ and in signal waveform and power estimation of deterministic processing versus probabilitistic averaging under conditions of a priori unknown signal waveform and input power, very low input $\mathbf{S} / \mathrm{N}$, short processing time and small number of sensors. For example, with input $\mathbf{S /}$ $\mathrm{N}$ in the region of -15 to $-30 \mathrm{~dB}$, order of 3 to 10 sensors, and 5 to 20 time sampling points, improvements in output $S / N$ on the order of 5 to 15 $\mathrm{dB}$ are achieved. These results are preceded by a summary of relevant deterministic properties of data sets and derivation of the deterministic algorithm used. Areas of further necessary work and implementation concepts are discussed. [Work supported by NUSC.]

\section{8:09}

U2. Generation of multiple target data sets. Homer P. Bucker (Code 5311, Naval Ocean Systems Center, San Diego, CA 92152)

Standard analysis of acoustic detection systerns is based on the sonar equation in terms of array signal gain, array noise gain, signal level, noise level, etc. In most cases, results from the sonar equation are too optimistic because of real ocean effects such as multipath arrivals, array bending, and environment induced fluctuations. In addition, most modern highresolution techniques, such as cross-sensor processing, cannot be analyzed in terms of the sonar equation because needed quantities such as directivity index cannot be defined. To solve this problem a more basic approach is required. Experiments should be conducted using multiple sources for tests in areas where the acoustic system will operate. Then it is a simple matter to determine the performance of the acoustic system. Because a multisource experiment may be too expensive, a good approximation to the desired experiment can be obtained by combining data collected at different times during a single source experiment. An example will be shown using data collected during a recent sea test.

\section{8:13}

U3. Doppler properties of pulse bursts. Henry Cox and Michael Frentz (Bolt Beranek and Newman Inc., 1300 North 17 th Street, Arlington, VA 22209)

Impulsive sound sources are attractive because they can generate very high energy levels in short pulses with good range resolution properties. However, short pulses have relatively poor Doppler resolution properties and in a number of measurement applications Doppler resolution is important. This paper discusses the design of sequences of pulses called bursts which are processed coherently. New results are presented for the Doppler properties of nonuniformly spaced pulses. The effectiveness of coarse quantization achieved by varying the number of sources per pulse in achieving good side-lobe behavior in the Doppler domain for uniformly spaced pulses is illustrated in several examples. Results for narrow-band pulse bursts $(2 V T W / C<1)$ are generalized to broadband pulse where the wideband ambiguity function must be used.

\section{8:17}

U4. Beampattern synthesis and computation for cylindrical arrays. Henry Cox, Robert Elmer, and John Thurston (Bolt Beranek and Newman Inc., 1300 N. 17th Street, Arlington, VA 22209\}

The synthesis and calculation of beampatterns for cylindrical arrays can be greatly simplified by factoring the problem into a line array of identical hoops under very mild restrictions on array geometry. For example, elements may be directive and hoops may be nonuniformly spaced. This result has particular significance for large arrays of many elements. The design approach consists of using one of several techniques to design a good hoop beampattern combined with a standard technique for designing a good line array beampattern. The technique is illustrated by application to an array of more than 2000 directional elements where other design techniques are impractical and straightforward computation of beampatterns is several orders of magnitude more time consuming. [Work supported by ONR.]

\section{$8: 21$}

U5. Hybrid phase-shift/time-delay digital beamforming. Robert A. Gabel and Richard R. Kurth (Sperry Research Center, 100 North Road, Sudbury, MA 01776)

Digital phase-shift beamforming of narrow-band signals from uniform colinear hydrophone arrays may be accomplished by computationally efficient algorithms which exploit the functional regularity of the required phase-shift operations. However, phase-shift beamforming is a valid approximation to delay-and-sum steering over only a limited bandwidth whose value depends on array aperture and steering angle. This paper describes a multiple-beam digital beamforming structure which is a phase-shift/time-delay hybrid and which an be designed for operating bandwidths in excess of the phase-shift limit. Much of the computational efficiency of colinear array phase-shift beamforming is retained in this approach, and it can offer substantial advantage over the alternative of delay steering by digital interpolation. This will be demonstrated by examples.

\section{8:25}

U6. Frequency-dependent refocusing in phase shift beamforming systems. James F. Lynch (Woods Hole Oceanographic Institution, Woods Hole, MA 02543), Bob Stokes, and Al Garcia (Applied Research Laboratories, The University of Texas at Austin, Austin, TX 78712) 
U14. Sonar array measurement facility. Frederick Reitz and Melvin Main (Gould Inc., Defense Electronics Division (DED), 6711 Baymeadow Drive, Glen Burnie, MD 21061)

DED has developed a facility for the accurate calibration of towed line sonar arrays which is small and noise immune enough that it can be installed at any site requiring such measurements. The facility consists of a series of cylindrical transducers submerged in a water tank. The array under test is pushed through the transducers and the free field voltage sensitivity of each channel is measured one-at-a-time by comparison against some of the transducers. The remaining transducers project acoustical energy into the water. This facility is an outgrowth of a concept developed by NRL/USRD [J. F. Zalesak and W. J. Trott, J. Acoust. Soc. Am. 62, 91-101 (1977)]. Within limits, the accuracy of this facility meets the requirements of the Navy's towed line arrays. Typically, between 20 $\mathrm{Hz}$ and $2 \mathrm{kHz}$, measurements are repeatable within $\pm 0.7 \mathrm{~dB}$ and correlate with the Navy's measurements within $\pm 0.7 \mathrm{~dB}$. During the past three years, the facility has performed over 3000 measurements and is presently under development as a product line.

\section{9:01}

U15. Very accurate production oriented hydrophone calibration systems. Frederick Reitz and Melvin Main (Gould, Inc., Defense Electronics Division (DED), 6711 Baymeadow Drive, Glen Burnie, MD 21061)

DED produces towed line sonar arrays for a large number of U.S. Navy applications. The fact that the performance of these arrays is very tightly specified and that DED produces hydrophones for these arrays by the tens of thousands each year has forced the development of production oriented hydrophone calibration systems meeting the following requirements: parameters measured-free field voltage sensitivity, relative phase; frequency range $-1 \mathrm{~Hz}$ to $2 \mathrm{kHz}$; measurement repeatability$\pm 0.2 \mathrm{~dB}$ max., $\pm 0.5 \mathrm{~dB}$ degrees max.; measurement correlation with Navy's standard facility (NRL/USRD)- $\pm 0.2 \mathrm{~dB}$ max.; temperature/ pressure-ambient. Automated measurement systems have been developed where the above hydrophone parameters are measured within closed, rigid air chambers. Measurements are made by comparison against acoustical standards which are built into each chamber. These syslems provide a well behaved, repeatable measurement over a wide frequency range. These measurement systems meet or exceed the above requirements and have been in use for approximately ten years.

\section{9:05}

U16. A pseudorandom noise source, programmable in center frequency and bandwidth. Dixon W. Coulbourn (Applied Research Laboratories, The University of Texas at Austin, Austin, TX 78712)

A source of pseudorandom (PR) noise with switch-selectable center frequency and bandwidth is discussed. PR noise is generated by shift register encoders. The noise is filtered at baseband before being heterodyned to form uncorrelated symmetric sidebands of a suppressed carrier. The carrier is generated by an accumulator frequency synthesizer employing a multiplier-accumulator chip. The chip makes possible use of various crystal frequencies and allows for a variety of frequency step sizes.
U17. Spectral broadening in turbulent wakes and jets. Nicholas P. Chotiros and C. Robert Culbertson (Applied Research Laboratories, The University of Texas at Austin, Austin, TX 78712)

A continuous acoustic signal propagating through turbulence is scattered by variations in the flow velocity. The scattered signal is Doppler shifted in a random manner due to the turbulent motion of the scatterers, thus giving rise to spectral broadening. Since the scattered component is usually quite small in underwater acoustics, the method of small perturbations was used. A theoretical estimate of the power spectrum of the scattered signal was derived for a sound wave propagating in a wake, and also for the case of propagation in a jet. Some experimental results were obtained, and compared with the theoretical estimates. [Work supported by Naval Sea Systems Command.]

\section{9:13}

U18. Ambiguity design for the recognition of spatially extended acoustic scatterers. D. W. Ricker (Applied Research Laboratory, P.O. Box 30, State College, PA 16801)

Acoustic scatterers which have spatial extent in range $(R)$ and azimuth $(\theta)$ space may be mapped into an equivalent delay $(\tau)$ and frequency $(\phi)$ space if they are observed with an active sonar which has a relative crossrange velocity component. It is shown that the mean-square oulput of a simple correlation receiver may be maximized by seeking signal waveforms whose ambiguity functions most closely match the support of the scatterer image as mapped into $(\tau, \phi)$ space. Spatially extended scatterers may be recognized and an estimate of their shape and orientation may be obtained by a comparison of receiver responses to waveforms whose ambiguity functions have distinct support in $(\tau, \phi)$.

\section{9:17}

U19. Optimizing the performance of a quantizer. William A. Struzinski (Code 3213, Naval Underwater Systems Center, New London, CT 06320)

In a previous paper [W. A. Struzinski, J. Acoust. Soc. Am. Suppl. 170, S16 (1981)], a mathematical model was developed to compute the OR-ing loss for quantizers followed by an OR-ing device and an accumulator. The quantizer investigated was a three-bit device that extended from the noiseonly mean $m_{0}$ to $m_{0}+2 \sigma$. The subject paper examines different spans of the same three-bit quantizer. It is determined that the best performance results when the quantizer extends from the noise-only mean $m_{0}$ to $m_{0}+3 \sigma$. The OR-ing loss and the input SNR requirements to the $O R$-ing device are computed for the $m_{0}$ to $m_{0}+3 \sigma$ quantizer. These results are compared to those of the $m_{0}$ to $m_{0}+2 \sigma$ quantizer. It is concluded that a quantizer spanning the range $m_{0}$ to $m_{0}+3 \sigma$ is most beneficial for small alarm probabilities, small number of samples in the accumulator, and higher number of channels OR-ed. [Work supported by the Naval Sea Systems Command.] 
DADE AND FLORIDA KEYS ROOMS, 8:45 TO 10:50 A.M.

\author{
Session V. Physical Acoustics IV: Nonlinear Acoustics
}

\author{
Mark B. Moffett, Chairman \\ Naval Undenwater Systems Center, New London, Connecticut 06320
}

Chairman's Introduction-8:45

\title{
Contributed Papers
}

8:50

V1. Finite amplitude method for the determination of the acoustic nonlinearity parameter $B / A$. Wesley $\mathrm{N}$. Cobb (Applied Mechanics, Yale University, New Haven, CT 06520)

The acoustic nonlinearity parameter $B / A$ is determined using a method based on the finite amplitude distortion of a sine wave emitted by a piston. We measure the growth of the second harmonic component of this wave using a piston receiver, which is coaxial with and the same size as the source. In order to determine $B / A$, the experimental measurements are compared to a theory which incorporates the nonlinearity parameter. The theory developed for this study accounts for the influence of both diffraction and attenuation on the experimental measurements. For this reason, the method is more accurate than previous techniques that employ plane wave theory for a lossless medium. To test the measurement method, we compare the experimental results for $B / A$ in distilled water, ethylene glycol and glycerol to established values. The agreement between these values suggests that the measurement accuracy is plus or minus $4 \%$ for common liquids. [Work supported by ONR.]

9:05

V2. Effective acoustic nonlinear parameter $(B / A)$ of a mixture. Robert E. Apfel and Wesley N. Cobb (Applied Mechanics, Yale University, New Haven, CT 06520)

Wederive an expression for the effective nonlinearity parameter, $B / A$, of a mixture for which the density, compressibility, $B / A$, and mass (volume) fraction of each component is known. We then compare the predictions with experimental results reported in the literature and with our own experimental results for compositions ranging from solutions to tissues. Finally, we attempt to rationalize differences between predictions and experimental results, where they exist. [Work supported by U.S. Office of Naval Research.]

\section{9:20}

V3. Rapid cavitation in water induced by the reflection of shock waves. Bruce T. Unger and Philip L. Marston (Department of Physics, Washington State University, Pullman, WA 99164]

Negative pressures were produced in distilled water by refiecting shock pulses of $11 \mathrm{MPa}$ off a quasifree water-Mylar-air interface. The incident pulses, created by conventional impact techniques, were typically $1.7 \mu \mathrm{s}$ in duration. An interferometer measured the displacement of the interface from which the velocity history of the Mylar could be extracted. Tension is created in the water when rarefaction waves collide. The tension results in cavitation which is evidenced by a rapid rebound of the Mylar's velocity. Difficulties experienced in previous experiments [P. $L$. Marston and G. L. Pullen, in Shock Waves in Condensed Matter, 1981, edited by W. J. Nellis et al. (AIP, New York, 1982), pp. 515-519] have been overcome by reducing the thickness of the Mylar which initially constrains the water. Velocity histories give a clear indication of cavitation in the sample; the abrupt tension being relieved by bubble formation within $1 \mu \mathrm{s}$. Also evident is reverberation within the spalled layer of the pressure wave radiated by the bubbles. The results suggest an apparent dynamic tensile strength $>5 \mathrm{MPa}$ for moderately clean water. [Work supported by ONR and the American Chemical Society Petroleum Research Fund. Marston is an Alfred P. Sloan Research Fellow.]

V4. Finite-amplitude waveforms produced by a circular piston projector. Mark B. Moffett (Naval Underwater Systems Center, New London, CT 06320) and Jerry H. Ginsberg (Georgia Institute of Technology, Atlanta, GA 30332)

Measurements were made of the waveforms produced at six different locations on the axis of a $0.51-\mathrm{m}$-diam projector driven at $60 \mathrm{kHz}$ in the NUSC/Newport large acoustic tank facility. The locations were at the last three pressure maxima in the nearfield, a quasifarfield point at $5 \mathrm{~m}$, and two farfield positions at $10 \mathrm{~m}$ and $15 \mathrm{~m}$. The projector was driven at several levels, and yielded waveforms ranging from sinusoidal at the lowest levels and shortest ranges to shock formation at the highest levels and longer ranges. Two different hydrophones were used, but neither had a flat enough response to avoid ringing when shocks were present. The waveforms exhibit the asymmetry (sharp pressure peaks and rounded pressure troughs) previously observed by Browning and Mellen [J. Acoust. Soc. 44, 644-646 (1968)] and predicted by recent work of Ginsberg which accounts for diffraction as well as nonlinear propagation effects. [Work supported by ONR Code 425 UA.]

9:50

V5. Pulsed parametric array revisited. D. H. Trivett (Naval Research Laboratory, Washington, DC 20375) and Peter H. Rogers (Office of Naval Research, Arlington, VA 22217)

In a previous paper [J. Acoust. Soc. Am. Suppl. 1 71, S30 (A)] we presented the results of an investigation of the pulsed parametric array. We found that while the primary pulse exited the transducer a sum and difference frequency signal was scattered. However, once the interaction region was freely propagating (i.e., both boundaries moving at the sound speed) no further scattered signal was generated. This results in the observed scattered signal having a pulse length equal to the primary pulse length and the appearance of direct radiation from the face of the transducer. We also concluded that the directivity pattern, identical to the cw parametric array, was not due to a volume distribution of virtual sources. On re-examination this conclusion has been found to be incorrect. In this paper we demonstrate that the observed signal at a given farfield point is generated by a volume distribution of virtual sources, as long as contributions to the field at that point are still being received from the stationary boundary. Once signals from the stationary boundary cease there is no further scattering to that point. The extent of the scattering region is dependent upon the primary pulse length and the observation angle and is given by $L_{\text {eff }}=L /(1-\cos \phi)$, where $L$ is the primary pulse length and $\phi$ is the observation angle. This result may have some practical applications. 
fractive and nonlinear effects. Solutions are obtained for harmonic and difference frequency generation, under conditions (1) large ka, (2) small aperture angles, and (3) quasilinear interaction (strong shocks precluded). The solutions furnish computations on the phase and amplitude of both primary and secondary components in propagation along the acoustic axis and in the focal plane. Of particular interest are the phase shifts that are observed in passing through the region of geometrical focus. In general, focal phase shifts for difference frequency sound are not usually observed, while focal phase shifts for harmonic radiations are less pronounced that that experienced by linear radiations. Experimental results are presented on underwater measurements conducted with an $f / 2$ lens coupled to a dual, interleaved primary array operating at 373 and 403 $\mathrm{kHz}$. The measured data support the utility and validity of the analytical model. In particular it is shown that both diffractive and nonlinear effects are important in the focal region, especially for the harmonic radiations. The measurements also confirm the effective focusing of the difference frequency radiation for the present configuration, in that the width of the beam in the focal plane becomes quite narrow (approximately $1 / 3$ of a difference frequency wavelength). (Work supported by the Office of Naval Research.] ${ }^{\text {a) }}$ On leave from G.E.R.D.S.M., Le Brusc, France.

\title{
10:35
}

V8. Parametric sonar as applied to high resolution boundary scattering measurement. William L. Konrad, William I. Roderick, and Lynn F. Carlton (Naval Underwater Systems Center, New London, CT 06320)

\section{0:20}

V7. The field of a focused nonlinear source.T. G. Muir and B. G. Lucas ${ }^{\text {a) }}$ (Applied Research Laboratories, The University of Texas at Austin, Austin, TX 78712)

Theoretical and experimental results on focused, nonlinear radiations are presented. The theory is developed with a parabolic approximation to an inhomogeneous Helmholtz wave equation, and accounts for both dif-

The use of the parametric acoustic source with its narrow beam and lack of sidelobes permits measurement of sea surface and bottom forward and back scatter to very low grazing angles. Measurements using conventional sources are often contaminated by sidelobe or other unwanted returns which result when unavoidably large boundary areas are illuminated. This paper describes the design and performance of one parametric source which has been employed for such measurements. [Work supported by NAVSEA and NORDA 530.]

\section{Session W. Psychological Acoustics III: Animal Echolocation and Human Psychophysics (Poster Session)}

\author{
James W. Hicks, Chairman
}

\section{Institute for Advanced Study of the Communication Processes, University of Florida, Gainesville, Florida 32611}

\author{
Chairman's Introduction-8:45
}

\begin{abstract}
All posters will be displayed from 8:50-10:50 A.M. To allow all contributors an opportunity to see other posters, contributors of odd-numbered papers will be at their posters from 8:50-9:50 and contributors of evennumbered papers will be at their posters from 9:50-10:50.
\end{abstract}

W1. Recovery of spatial tuning of auditory neurons in the owl's optic tectum. Stephen J. Leibovic and Eric I. Knudsen (Department of Neurobiology, Stanford Medical School, Stanford, CA. 94305)

Monaural occlusion alters binaural sound localization cues. Nevertheless, young barn owls learn to localize sounds after eight weeks of occlusion. We investigated effects of monaural occlusion on responses of space-tuned neurons in the optic tectum. In normal birds, $85 \%$ of cells in deep layers respond to sound and light (bimodal); the rest respond to sound only. None respond to light only. Bimodal cells have coincident auditory and visual receptive fields and space for both modalities is mapped onto the tectum. Acute ear plugging abolished the auditory responsiveness of tectal cells. In contrast, after 8 to 14 weeks of occlusion, tectal cells were responsive and space-tuned. Twenty recordings from su- perficial tectal layers revealed all normal receptive fields. In deeper layers ( 43 recordings), $50 \%$ of the cells were bimodal, $40 \%$ responded to light only and $10 \%$ to sound only. Although the auditory fields were elongated vertically, they were normal in their horizontal dimensions. Moreover, the auditory and visual fields of single neurons were horizontally aligned. The recovery of auditory spatial tuning and the alignment of auditory and visual fields indicates that the binaural stimulus requirements of these neurons changed in an adaptive manner in response to altered binaural input.

W2. The external ears as receiving antennas in echolocating bats. J. A. Simmons (Institute of Neuroscience, University of Oregon, Eugene, OR 97403) 
The external ears of bats appear to vary greatly in size and shape, but they in fact have dimensions specifically related to the structure of sonar sounds in different species. They determine the directionality of hearing and produce cues for vertical target localization. The pinna of broadband (FM) bats is equal in length to the wavelength of the lowest first-harmonic frequencies in the sonar signals, even if the bat only broadcasts higherorder harmonics into the air. The bladelike tragus focuses the directional sensitivity of hearing more sharply and cleanly to the front than the pinna alone can achieve. It also produces secondary reflections within the external ear which encode target elevation and which are necessary for accurate vertical localization of targets. Narrow-band (CF) bats have a pinna 5 to 8 times longer than the wavelength of predominant signal frequencies and have sharper directional sensitivity than broadband bats which have a pinna only 3 to 4 times the predominant wavelengths. The configuration of the external ear matches the timing of tragus reflections to the period (autocorrelation) structure of FM sonar sounds to avoid average-phase ambiguity in perception of target elevation.

W3. Fcho sensitivity as a function of target range: Automatic gain control in eqholycating bats. S. A. Kjck (Institute of Neuroscience, University of Ortogytygene, OR 97403)

The sensitivity of the echolocating bat, Eptesicus fuscus, for detecting sonar echoes at different delays (corresponding to ranges of less than $3 \mathrm{~m}$ ) was measured in a two-choice psychophysical task. The stimuli were either electronically reproduced, delayed replicas of the bat's own sonar sounds or were tone-bursts triggered by the bat's sounds. The threshold for echo detectiôn ât delays for ranges of $3 \mathrm{~m}$ or more is about $0 \mathrm{~dB}$ SPL, but it rises for shorter delays, for example, to $35 \mathrm{~dB}$ SPL at the delay for 25 $\mathrm{cm}$. The bat's middle-ear muscles contract during vocalization and are still relaxing when echoes return from targets at close ranges, accounting for most of the elevation of thresholds. The threshold shift at different ranges corresponds to the change in echo strength from a target at different ranges due to spreading losses and atmospheric attenuation. The bat thus regulates its sensitivity to echoes to a constant echo strength above threshold as it approaches targets. The mechanism is a delay- or rangerelated change in threshold.

\section{$+4$}

:

W4. Beluga whale echolocation signals in two different ambient noise environments. Whitlow $W$. L. Au, Donald A. Carder, Ralph H. Penner, and Billy L. Scronce (Naval Ocean Systems Center, San Diego, CA 92152 and Kailua, HI 96734)

The echolocation signals of a Beluga whale (Delphinapterus lecus) were measured, first in San Siego Bay, and later in Kaneohe Bay, Oahu, Hawaii, while the animal was performing a target detection task. The ambient noise of both bays is dominated by snapping shrimp, with the noise level in Kaneohe Bay typically $20 \mathrm{~dB}$ greater than in San Diego Bay. In San Diego Bay, the animal emitted clicks with peak frequencies between 40 and $60 \mathrm{kHz}$, and bandwidths between 15 and $25 \mathrm{kHz}$. When the animal was moved to Kaneohe Bay, it used higher frequency clicks with peak frequencies between 100 and $120 \mathrm{kHz}$, and bandwidths between 20 and $40 \mathrm{kHz}$. The shift in the peak frequencies was probably caused by the difference in the ambient noise environment between the two sites, and may represent an attempt by the animal to optimize the signal-to-noise ratio during the target detection task. The whale also emitted signals with higher source levels and source energy flux densities in Kaneohe Bay than in San Diego Bay, even though the detection task in Kaneohe Bay was relatively simple and the task in San Diego Bay was relatively difficult.

W5. Directional hearing in the Atlantic bottlenose dolphin (Tursiops truncatus). Whitlow W. L. Au and Patrick W. B. Moore (Naval Ocean Systems Center, P.O. Box 997, Kailua, HI 96734)

The directional hearing sensitivity of an Atlantic bottlenose dolphin was measured behaviorally in the vertical and horizontal planes for puretone frequencies of 30,60 , and $120 \mathrm{kHz}$. The vertical beam-pattern results were reported previously [Moore and Au, J. Acoust. Soc. Am. Suppl. 170 , S85 (1981)]. Measurements in the horizontal plane were performed with the dolphin stationing in a bite plate and with two noise sources located $\pm 20^{\circ}$ on either side of the bite plate, $3.5 \mathrm{~m}$ away. The levels of the noise sources were held constant and the masked thresholds were determined by varying the level of the signal source at different azimuths. The horizontal beam patterns, which were directed forward, were fairly symmetrical about the major axis and became narrower as the frequency increased. Using the beam patterns measured in the vertical and horizontal planes, the receiving directivity index was calculated and found to be approximately 10,15 , and $21 \mathrm{~dB}$ for frequencies of 30,60 , and $120 \mathrm{kHz}$, respectively. A two-element rectangular transducer array was used to model the dolphin's directivity index; each element having an effective area of $\mathbf{8 . 1}$ $\mathrm{cm}^{2}$. The frequency dependency of the dolphin's directivity index was found to be very similar to the linear array model.

W6. Masked pure-tone thresholds of the bottlenosed dolphin (Tursiops truncatus) at extended frequencies, Patrick W. B. Moore and Whitlow W. L. Au (Naval Ocean Systems Center, P.O. Box 997, Kailua, HI 96734)

Masked underwater pure-tone thresholds were obtained for a bottlenosed dolphin (Tursiops truncatus) using an "up-down staircase" method of stimulus presentation and a "go/no-go" response procedure. Thresholds were determined at two continuous broadband $1 \mathrm{kj}$ ing noise levels at test frequencies of $30,60,90,100,110,120$, and $1 \mathrm{kH}$ The Critical Ratio (CR), defined as the ratio of signal power to noise spectrum level at masked threshold, was calculated for both noise levels. A psychometric function relating CR's to frequency showed parallel functions with CR's previously reported for the dolphin [Johnson, J. Acoust. Soc. A 965-967 (1968)] for frequencies up to $100 \mathrm{kHz}$. Results for 30,60 , 98 , and $100 \mathrm{kHz}$ were $31,32,45$, and $40 \mathrm{~dB}$, respectively, supporting previous findings for the dolphin. Results beyond $100 \mathrm{kHz}$, where no other values had previously been obtained, show a sharp increase at $110 \mathrm{kHz}$ (51 dB) followed by a slight decline at $120 \mathrm{kHz}$ (46 dB). Generally, the CR function suggests that the frequency resolving ability of this echolocator is not optimal at these higher frequencies even though peak energies of the outgoing echolocation pulse have been recorded in this higher range.

\section{W7. Abstract withdrawn.}

$\pm$

W8. Temporal resolution in normal-hearing and noise-exposed chinchillas. Diane Giraudi-Perry (Albert Einstein College of Medicine, Bronx, NY 10461), Richard J. Salvi, and Donald Henderson (Callier Center for Communication Disorders, Dallas, TX)

Auditory temporal resolution has been shown to deteriorate with sensorineural hearing loss (Boothroyd, 1973; Fitzgibbons, 1979); however, there is considerable intersubject variability in human studies. The purpose of the present experiment was to investigate the effect of stimulus presentation level on the gap detection thresholds obtained from chinchillas during various amounts of noise-induced asymptotic threshold shift 
(ATS). ATS was induced with an octave-band of noise centered at $500 \mathrm{~Hz}$ and having a SPL of $75,85,95$, and $100 \mathrm{~dB}$. Gap detection thresholds were unaffected by the 75-dB SPL noise exposure. However, during the 85-dB SPL noise exposure, gap detection thresholds were longer than normal if compared at the same sound pressure levels, but within normal limits if compared at similiar sensation levels. At ATS for the 95 or 100 -dB SPL noise exposures, gap detection thresholds were significantly wider than pre-exposure measures regardless of whether the comparison was in terms of sound pressure level or sensation level. The results show that there is an orderly breakdown in temporal resolution as the degree of noise-induced ATS increased. [Supported in part by grants from NIH (NS 1676), NIOSH (OH-00364), and the U.S. Army Medical Research and Development Command (DAMD 17-80-0133)]

W9. No hysteresis effect in adaptive masked thresholds. Daniel L. Weber and Betty L. Habler (Boys Town Institute, 555 North 30th Street, Omaha, NE 68131)

We measured forward-masked thresholds for a $10-\mathrm{ms}, \mathrm{l}-\mathrm{kHz}$ sinusoidal signal as a function of masker level for both sinusoidal and narrowband $(60 \mathrm{~Hz}$ wide) noise maskers at five masker frequencies $(0.6,0.8,1.0$, 1.15 , and $1.25 \mathrm{kHz}$ ). We measured these growth-of-forward-masking functions in the conventional fashion (determine the threshold level of the signal for a fixed level of the masker) and with the converse procedure (determine the masker level necessary to just mask a given signal). A common growth-of-masking function describes the results of both procedures for a given masker type and frequency, i.e., identical stimuli lead to identical performance regardless of whether one varies signal level or masker level. The growth-of-masking functions for different masker frequencies show varying degrees of nonlinearity. The nonlinearity of the growth-ofmasking functions underlies the discrepancies which arise between masking pattern and tuning curve data. These discrepancies do not arise because of a "hysteresis effect" between the psychophysical procedures. [Research support by NIH and NSF.]

W10. A quantal first effect of pitch shift? Edward M. Burns (Department of Audiology and Speech Sciences, Purdue University, West Lafayette, IN 47907)

Langer [Exp. Brain Res. 44, 450-454 (1981)] has proposed a model for the perception of the "periodicity pitch" of AM tones based on the neuronal cross correlation of subharmonics of the carrier frequency with an internal oscillator with a period of $0.4 \mathrm{~ms}$ which is phase locked to the modulation envelope. The evidence for the $0.4-\mathrm{ms}$-period internal oscillator comes from two sources: (1) physiological measurements on single neurons from the midbrain of the Guinea fowl, and (2) psychophysical measurements on the pitch perception of AM tones by humans. The pitch experiment is a version of the classic "first effect of pitch shift" experiments wherein observers match the pitch of an AM tone whose carrier frequency is shifted, in constant $(\mathrm{Hz})$ increments, over a range corresponding to about twice the modulation frequency. The primary difference between Langner's experiment and previous experiments is in the resolution of carrier shifts (increments of $10 \mathrm{~Hz}$ ). Langner reports that, in contrast to the smooth shift in pitch estimates obtained in previous studies, many observers show steplike shifts, with the step size corresponding to a change in the period of the pitch estimate of about $0.4 \mathrm{~ms}$. In an effort to replicate this result, we have obtained similar measurements on a large number of highly-trained observers. To date we have found no evidence of quantal shifts in pitch of the type described by Langner.

W11. An acoustic approach to diver navigation: Parameter selection and training effects. J. W. Hicks, Jr., H. Hollien, and B. Klepper (Institute for Advanced Study of the Communication Processes, University of Florida, Gainesville, FL 32611

Recently Hollien, Hicks, and Klepper (1981) presented data concerned with the development of an underwater diver navigation system based on the projection of an acoustic signal through an underwater range. It was found that a multiple beacon source, energized sequentially in a linear fashion, generates an Underwater Auditory Phi Phenomenon (UAPP). This multiple beacon configuration, when used in combination with a thermal noise source, produces an acoustic field which divers can localize and swim to from a distance of $150 \mathrm{~m}$. As part of a coordinated research program designed to develop and refine this acoustic navigation system, further investigations were carried out to (1) delineate more precisely the types of signals that were best suited for the production of the UAPP percept in divers, and (2) determine the effects of training on divers' abilities to navigate through the markerless space that is characteristic of the underwater environment. The results of this investigation demonstrate that divers improve their navigational skills quite rapidly, and even previously uninitiated divers learn to accurately home on the target within five or six trials. Furthermore, it appears that a band-limited thermal noise source provides the best acoustic navigation signal. [Work supported by Florida Sea Grant award R/OE-12.]

\title{
Session X. Memorial Session for John C. Johnson
}

\author{
Jiri Tichy, Chairman
}

Graduate Program in Acoustics, Pennsylvania State University, University Park, Pennsylvania 16802

Chairman's Introduction-9:00

Invited Papers

X1. Natural modes, coupled modes, and transients of vibratory systems. Eugen J. Skudrzyk (Applied Research Laboratory, The Pennsylvania State University, P.O. Box 30, State College, PA 16801)

Resonances of a point driven vibrator occur when the reflected waves reach the driver in phase with the outgoing wave, antiresonances occur when these two waves are in antiphase. The frequency response for the outgoing wave field determines the geometric mean line through the frequency response curve of the vibrator. Reflections at the boundaries of the vibrator, at ribs and appendages, or at material variations, have no effect on 
the "mean-line response." Plate modes are scalar functions of the position. In contrast, shell modes are represented by three-dimensional functions. But they are also described by uncoupled and orthogonal mode functions. Isolated vibrating systems always have orthogonal modes. It may sometimes be convenient to describe a vibrator by mode functions of some simpler vibrator. Every mode then is represented by a sum of mode functions which are coupled (e.g., plate with a mass load). The transients of a complex vibrator can be built up from the transients of its modes. An understanding of the theory of transients is important, for instance, when frequency modulated pulses are generated; increasing the damping may lead to long duration transients; the aim here is to cancel the switching-on transient with the switching-off transient which can only be done if damping is small. The theory of natural modes and transients will be illustrated by practical examples. [This work was sponsored by the Office of Naval Research, Code 474.]

9:35

X2. John C. Johnson-Applied Research Laboratory Director and Penn State Professor. L. R. Hettche (The Applied Research Laboratory, The Pennsylvania State University, University Park, PA 16802)

\title{
9:55
}

X3. J. C. Johnson and the U.S. Navy. Carey D. Smith (Naval Sea Systems Command, NSEA 63R, Department of the Navy, Washington, DC 20362)

10:05

X4. J. C. Johnson's contribution to Noise Control Engineering. W. W. Lang (IBM Acoustics Lab., C18/704, P.O. Box 390, Poughkeepsie, NY 12602)

10:20

X5. The Role of J. C. Johnson in the physics community. R. T. Beyer (Department of Physics, Brown University, Providence, RI 02912)

WEDNESDAY MORNING, 10 NOVEMBER 1982

PINELLAS ROOM, 9:00 TO 10:50 A.M

\section{Session Y. Architectural Acoustics IV: Bull Session on Research in Architectural Acoustics \\ David Lubman, Chairman}

D. Lubman and Associates, 2217 Vista Del Sol, Fullerton, California 92631

A forum for presentation and discussion of new scientific ideas, initiatives, and technical challenges in the field of architectural acoustics. Anyone wishing to present a topic should contact the Chairman in advance of the session.

\author{
Chairman's Introduction-9:00
}

Invited Paper

9:05

Y1. Peristyle-50 years. D. A. Harris (Owens Corning Fiberglas Corp., Fiberglas Tower, Toledo, OH 43659)

"A modern form of Greek Theater" is what Time magazine called it in January, 1933. The "Peristyle" concert hall, in Toledo, Ohio, formally opened to Leopold Stokowski conducting the Philadelphia Symphony Orchestra. Termed "a modern version of an open air theater." semi-circular tiers surround the orchestra section with the topmost row topped by ionic colonades. A generous stage, cork flooring, marble paneled walls, and gently domed ceiling that extends beyond the colonades complete the acoustical envelope. A concealed lighting system gives an effect from "bright warm sunlight" to "a night sky shimmering with moonlight." Opening to harsh criticisms of both the architecture and acoustics, the Peristyle, a part of the Toledo Museum of Art and a gift of Edward Drummond Libby, is generally considered to be an excellent hall for listeners. Musicians are not universally in agreement. A gentle blending of the orchestra can be found at nearly every seat. Yet the players complain of not being able to hear each other. To this day, electronic amplification is unnecessary even for verbal announcements. With only slight modifications over decades, the Peristyle acoustical attributes will be described using modern day technology and equipment with the hall in its present state.

9:30 to 10:50

Open Forum 


\title{
Session Z. Musical Acoustics II: Melody
}

\author{
Daniel W. Martin, Chairman \\ Baldwin Piano and Organ Co., 1801 Gilbert Avenue, Cincinnati, Ohio, 45202
}

Chairman's Introduction-9:00

\section{Contributed Papers}

9:05

Z1. Integration of melody and text in memory for songs. Mary Louise Serafine, Robert G. Crowder (Department of Psychology, Yale University, New Haven, CT 06520), and Bruno H. Repp (Haskins Laboratories, 270 Crown Street, New Haven, CT 06510)

Subjects with varying musical experience listened to a series of 24 brief excerpts from relatively unfamiliar folksongs. Subsequently, test series of 20 similar excerpts was presented and the subjects indicated for each excerpt whether they had heard that precise song before or, if not, whether they had heard the tune or the words. The test items included (a) old songs, (b) new songs, (c) old tunes with new words, (d) new tunes with old words, and (e) old tunes with "mismatched" old words that had previously been part of a different song. The results showed that old songs were recognized very well, whereas recognition of old tunes with new or mismatched old words was very poor, and recognition of old words with new or mismatched old tunes was good but nevertheless below the accuracy achieved for old songs. Thus, Thelody and text of songs seem to be remembered in an integral fashion nf as separate entities. [Work supported by NSF.]

9:25

22. "Figural" and "tonal" aspects of melody discrimination. Anthony J. Watkins (Department of Psychology, Reading University, Reading, England RG6 2AL)

Tone sequences were synthesized using a random process constrained to generate relatively close approximations to the tonal relationships found in melodies which occur in music. In control sequences these constraints are systematically inverted. Other melody variables were fixed (e.g., rhythnfyr completely randomized. The perceptual task involved discrimination between "same transposed" and "different transposed" melody pairs. Alterations which changed the "contour" of the melody were compared with those which preserved contour. We find that contour violation and a low "Sth span" of the melody intervals aid discrimination, and these operate independently. We also examined diatonic scale, contour and tuning, the latter being equal temperament ( 100 cents per semitone), "micro" (60 cents per semitone) or "macro" (140 cents per semitone). A discrimination advantage for melodies based on a diatonic scale is only found for equal temperament tuning, whereas contour violation aids discrimination regardless of tuning. We conclude that contour is a general perceptual property of serial auditory patterns ("figural"), whereas scale and Sth span are specific to sequences heard as music ("tonal").

\section{9:45}

23. Melodic octave tuning; separable dimensions of perception. Scott Makeig and Gerald Balzano (Department of Music, University of California at San Diego, La Jolla, CA 92037)

Ascending and descending digitally synthesized pure-tone melodic octaves jn the range $250-1500 \mathrm{~Hz}$, varying width from $1180_{q}$ to $1220_{q}$ were pressinted to nine highly musical subjects who were asked to judge (1) degree of octave mistuning, and (2) whether the second tone of the pair was sharp or flat. Results confirm the existence of so-called "octave stretch" previously reported in the literature. However, the two tasks were found to be perceptually dissimilar in degree of octave stretch, variability, and dependence on register. Consistent with results of Lindquist and Sundberg (VIIth Int. Congr. Acoust., 1971] preferred "octave stretch" for the "sense of mistuning" judgments was very small Gabout 2.5\%) for low register octaves. An additional significant finding was that melodic octaves whose second tone was flat relative to an adjudged "in tune" octave were called more "mistuned" than octaves whose second tone was equally sharp. Together, these results imply that perception of octave tuning does not amount to a one-dimensional judgment of log frequency difference, but is multidimensional in nature.

\section{0:05}

ZA. Automatic identification of musical chords. Daniel W. Martin (Baldwin Piano \& Organ Company, Cincinnati, OH 45202)

Various electronic and computer systems, which have been developed during the last fifteen years for identifying musical chords automatically. will be reviewed and compared. The purpose in most cases has been to facilitate easy-play, automatic musical accompaniment for musical beginners on electronic musical instruments. However the means used could also help provide low-cost instrumentation for real-time recording of new musical compositions, improvisations, and arrangements.

\section{Session AA. Noise IV: Environmental Noise Propagation and Annoyance}

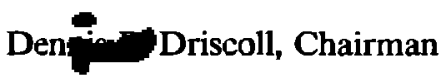

Standara Oil Company (Indiana), 200 E. Randolph Drive, Chicago, Illinois 60601

\section{Contributed Papers.}

\section{9:05}

AA1. Danish activities concerning noise in the environment. Fritz Ingerslev (The Acoustics Laboratory, Technical University, DK2800 Lyngby, Denmark)
The paper describes the administrative activities and the distribution between federal and local authorities. The importance of having a federal agency with highly qualified employees who can establish a superior national noise abatement strategy is stressed. The federal authority should represent the country in international collaboration. It is claimed that 
noise abatement will be diffuse and weak, if it is not based on a national strategy. The discussion of noise in the environment covers: external industrial noise, road traffic noise, and air traffic noise.The principles on which the maximum permissible Danish noise exposure levels are based will be explained. ISO 1996, 1st edition 1971, has played a decisive part. The maximum permissible Danish noise exposure levels for external industrial noise are strict and enforced for new plants and in connection with enlargement of existing plants. The regulations concerning road traffic noise are based on results of extensive interviews of persons living in areas with various exposure levels. The results of these interviews will be reported as well as the conclusions drawn.

AA2. Multiple-event airplane noise annoyance. Clemans A. Powell (NASA Langley Research Center, Hampton, VA 23665)

Two experiments were conducted in which subjects in a simulated living room environment judged the annoyance of sessions of airplane noise which contained different noise levels and numbers of flyovers. In the first experiment one, two, or four high noise level flyovers occurred at the beginning, middle, or end of 30-min test sessions each of which contained a total of eight flyovers. In the second experiment, one, four or 16 flyover noises occurred in 15,30 , or 60 -min test sessions. The time-ofoccurrence of the high noise level flyovers in the sessions did not significantly affect annoyance, but annoyance increased with the number of such flyovers. Annoyance decreased with test session duration but increased with the total number of flyovers in the test sessions. These results support an average energy model better than total energy, annoyance decay, or peak noise level models.

\section{9:35}

AA3. Administering a "noise budget" for a civil airport. Dwight E. Bishop (Bolt Beranek and Newman Inc., P. O. Box 633, Canoga Park, CA 91305)

"Noise budgets" offer air carrier airports a means for achieving noise control without imposing rigid restrictions of numbers of operations or types of aircraft. This paper describes the application of noise budget concepts to actual administration of operations at a medium size air carrier airport. The airport's noise budget is based upon consideration of the noise-incompatible land use areas within a specified community noise equivalent level (CNEL) contour. However, budget controls for air carriers are expressed in terms of the "equivalent numbers of operations" where the equivalent number is based upon the number of opertions per day, evening, and night periods and the relative noise levels produced by different aircraft. The relative noise levels are derived from data from the airport's permanent noise monitoring system. Samples of the budget analyses that are undertaken to evaluate changes in air carrier schedules will be presented.

$$
\text { 9:50 }
$$

AA4. Outdoor sound propagation over ground with several impedance discontinuities. M. Reada Bassiouni (Acoustic Technology, Inc., 240 Commercial St., Boston, MA 02109]
Extensive studies have been made on the outdoor propagation of sound over a surface of finite impedance as well as over a surface with an impedance discontinuity. Under realistic field conditions, the ground surface over which sound is propagating has several discontinuities. This paper deals with the propagation of sound from a well-defined sound source over a surface characterized by a finite number of infinitely long parallel regions of ground with homogeneous impedance within each region, but the impedance varies from one region to another. The sound source itself generates one tone at considerably higher levels than the higher order harmonics. The analytical results are presented in comparison to experimental measurements obtained from testing conducted under actual field conditions. Factors such as topography and meteorology, which would otherwise contribute to the excessive attenuation, have been omitted in order to isolate the effects of a finite number of impedance discontinuities. Because the analytical study has been validated by field results the findings will improve prediction methods of environmental noise from sources of discrete tones (e.g., high power transformers, I.D. and F.D. fans, etc.) propagating over realistic outdoor ground conditions.

\section{0:05}

AA5. Community noise from air-cushioned landing craft. Richard E. Burke (Parsons Corp., 100 W. Walnut, Pasadena, CA 91124)

The effect of noise on neighboring communities resulting from operating the U.S. Navy's proposed Air Cushioned Landing Craft (LCAC) at an initial training site was predicted and evaluated in this preliminary impact assessment. Farfield noise levels of two prototype vehicles, each with a maximum gross weight of 160 tons, were measured under various station. ary and moving modes at the Naval Coastal Systems Center in Panama City, FL. These data and appropriate vehicle operating characteristics were entered into NOISEMAP, a noise contour generating computer program which had heretofore only been used in aircraft noise analyses. Four vehicles undertaking a total of 440 annual missions were found to generate a 65-dB DNL contour with an area of only $1 / 4$ square mile. Nonetheless, single event maximums over $65 \mathrm{~dB}$ predicted near residential locations were identified as potential areas of adverse impact on the community. [Work supported by the U.S. Navy.]

\section{$10 ; 20$}

AA6. Noise levels of Hong Kong marine craft. Richard E. Burke (Parsons Corp., 100 W. Walnut, Pasadena, CA 91124)

Noise measurements were made of jetfoils, hydrofoils, hovercraft, and nine other kinds of passenger and commercial marine craft operating in Hong Kong's Victoria Harbor for the purpose of predicting noise impacts expected from similar craft at a proposed offshore airport. Tape recordings of several passbys of each craft were analyzed to obtain characteristic time histories, spectra, and maximum, statistical, and equivalent noise levels. Results provide initial indication of main concerns expected from marine craft noise and suggest operational abatement measures which may be effective. [Work supported by the Hong Kong Civil Aviation Department.]

WEDNESDAY MORNING, 10 NOVEMBER 1982

SARASOTA AND ST. JOHNS ROOMS, 9:00 TO 10:35 A.M.

\title{
Session BB. Physiological Acoustics III: Cochlear Metabolism and Theory
}

\author{
Donald W. Nielsen, Chairman \\ Otological Research Lab., Henry Ford Hospital, 7036 Education \& Research Building, Detroit, Michigan 48103
}

Chairman's Introduction-9:00

\section{Contributed Papers}

BB1. Metabolic requirements of endocochlear potential (EP) T. Kobayashi, M. Rokugo, I. Thalmann, and R. Thalmann
(Department of Otolaryngology, Washington University Medical School, St. Louis, MO 63110) 
We have, in recent years, developed a method for arterial perfusion of the isolated inner ear of the guinea pig with "artificial blood," consisting of an emulsion of a perfluorocarbon oxygen carrier in a physiological salt solution, containing a high molecular weight polyol as emulsifier and plasma expander. Using this technique, we found that, in addition to $D$ glucose, mannose can sustain the EP at normal levels for prolonged periods, while L-glucose, fructose, and galactose are ineffective. We also demonstrated that lactate and pyruvate can support the potential [Kambayashi et al., Hear. Res. 7, 105-114 (1982)]. In subsequent studies using the same technique, we have found that acetate, acetoacetate, and betahydroxybutyrate can also maintain the EP at normal levels; however, glycerol and phosphoenolpyruvate, as well as respresentative amino acids such as glutamine, glutamate, and alanine could not support the EP. Experiments concerning the possible utilization of fatty acids by the stria vascularis are still inconclusive since the synthetic blood may lack adequate carriers for these substances. A different perfusion medium containing carrier proteins will be required for a definitive answer. [Supported by NIH.]

\section{9:20}

BB2. Profiles of free amino acids in cochlear structures. $R$. Thalmann, T. H. Comegys, and I. Thalmann (Department of Otolaryngology, Washington University Medical School, St. Louis, MO 63110)

Godfrey et al. [J. Histochem. Cytochem. 24, 468-470(1976)] reported levels of the putative amino acid transmitters aspartate, glutamate, glycine and GABA in cochlear structures. Moreover, Thalmann et al. [J. Acoust. Soc. Am. Suppl. 1 66, S47 (1979)] determined levels of aspartate and glutamate in cell layers of the organ of Corti. Both studies used catalytic fluorometric assays, in which one amino acid is determined at a time. Due to recent advances in high performance liquid chromatography, we have been able to determine profiles of the majority of common amino acids in different tissues of the inner ear. Our findings with respect to the putative amino acid transmitters agree with earlier studies. In the organ of Corti, for example, glutamate is abundant $(60-70 \mathrm{mmoles} / \mathrm{kg}$ dry weight), as previously shown. The putative neurotransmitters aspartate and glycine, as well as serine, glutamine, alanine, and phosphoethanolamine, are also present at considerable levels ( $8-30 \mathrm{mmoles} / \mathrm{kg}$ dry weight). The other amino acids measured range from 0.3 to $6.0 \mathrm{mmoles} / \mathrm{kg}$ dry weight. Data on other cochlear structures will be presented and implications of our findings discussed. [Supported by NIH.]

\section{9:35}

BB3. Alteration of furosemide ototoxicity. Leonard P. Rybak and Craig Whitworth (Department of Surgery, SIU School of Medicine, Springfield, IL 62702)

The present study was designed to investigate the effects of inhibitors of organic acid transport on furosemide ototoxicity. Chinchillas weighing 400-600 g were anesthetized with ketamine $45 \mathrm{mg} / \mathrm{kg}$ IM followed by pentobarbitol $30 \mathrm{mg} / \mathrm{kg}$ IM. Control animals were injected intravenously with $0.5 \mathrm{ml}$ of either phosphate buffer or normal saline $30 \mathrm{~min}$ prior to injecting furosemide $25 \mathrm{mg} / \mathrm{kg}$ IV. Experimental animals received an intravenous injection of either probenecid $50 \mathrm{mg} / \mathrm{kg}$ in phosphate buffer or novobiocin $50 \mathrm{mg} / \mathrm{kg}$ in saline $30 \mathrm{~min}$ prior to the injection of furosemide $25 \mathrm{mg} / \mathrm{kg}$ IV. The magnitude of change of EP did not differ in the experimental group pretreated with novobiocin prior to furosemide compared with controls. However, the experimental group which received probenecid prior to furosemide showed a striking reduction in the magnitude of EP change compared to both controls and the novobiocin treated experimental group. The time required for EP recovery did not significantly differ in the three groups although the group treated with probenecid did show a tendency for longer recovery times. [Work supported by NIH and Deafness and Research Foundation.]
BB4. Response phases of cochlear hair cells at low sound frequencies and their significance. J. J. Zwislocki (Institute for Sensory Research, Syracuse University, Syracuse, NY 13210)

Hair-cell excitation depends on the deflection of their stereocilia toward their kynocilium or basal body. Since the stereocilia of the outer hair cells $(\mathrm{OHCs})$ are embedded in the tectorial membrane, their deflection must be controlled by the shear displacement between this membrane and the reticular lamina. Inferring from cochlear microphonics, $\mathrm{OHCs}$ are depolarized at low frequencies when the basilar membrane is displaced toward scala vestibuli. Because of the geometry of the organ of Corti, this produces a radial displacement of the reticular lamina toward the limbus. The response phase requires that the tectorial membrane move radially either in phase or in phase opposition with the reticular lamina, but the latter possibility may be excluded on the basis of the mechanics of the system. Since, in general the depolarization of the inner hair cells (IHCs) does not follow the depolarization phase of $\mathrm{OHCs}$, their stereocilia cannot be solidly embedded in the tectorial membrane. The known experimental results are compatible with a viscoelastic coupling. Such a coupling may result from the viscosity and surface tension of the surrounding fluid. In addition, the assumption made earlier that at low frequencies at the location of IHCs the amplitude of the radial tectorial-membrane displacement is nearly the same as of the reticular lamina must be accepted. [Work supported by NIH.]

\section{0:05}

BB5. Model calculations of low-frequency intracochlear pressures and the cochlear input impedance of the cat and guinea pig. Shozo Koshigoe, Wai-Kwong Kwok, and Arnold Tubis (Department of Physics, Purdue University, West Lafayette, IN 47907)

Recent low-frequency measurements of intracochlear pressures and the cochlear input impedance of the cat [V. Nedzelnitsky, J. Acoust. Soc. Am. 68, 1676-1689 (1980); T. J. Lynch, III, V. Nedzelnitsky, and W. T. Peake, J. Acoust. Soc. Am. 72, 108-130 (1982)] and the guinea pig (A. Dancer and R. Franke, Hear. Res. 2, 192-205 (1980)] are shown to be in reasonable agreement with the results of cochlear model calculations. Included in the cochlear model are perilymph viscosity, the measured variation of the area of the scala vestibuli with distance from the stapes [P. Dallos, J. Acoust. Soc. Am. 48, 489-499 (1970)], and the finite impedance of the round window membrane. Approximate analytic expressions for the fluid pressures in the scalae are derived so as to explicitly exhibit the dependence of the model results on cochlear parameters. [This work was supported by NSF.]

\section{$10: 20$}

BB6. A nonlinear feedback model for the mechanical response of outerhair-cell sterocilia. Shozo Koshigoe, Wai-Kwong Kwok, and Arnold Tubis (Department of Physics, Purdue University, West Lafayette, IN 47907)

There is some experimental evidence that the mechanical cochlear function is strongly influenced by the efferent innervation of outer-haircell stereocilia (OHCS). [See, e.g., D. C. Mountain, Science 210, 71-72 (1980); J. H. Siegel and D. O. Kim, Hear. Res. 6, 171-182 (1982).] This suggests that the various nonlinear and active mechanical features of the cochlear response may be mainly rooted in the OHCS. We propose a specific nonlinear feedback model for the mechanics of the OHCS, which is a generalization of a linear model proposed by Mountain. It gives formally the combination of negative resistance and nonlinear damping used in previous calculations [S. Koshigoe and A. Tubis, J. Acoust. Soc. Am. Suppl. 1 71, \$17 (1982)]. The parameters of the model are determined so as to account for experimental measurements of the basilar membrane response as well as stimulated and spontaneous emissions in the ear canal. [This work was supported by NSF.] 


\section{Session CC. Speech Communication IV: Modeling}

John F. Boehm, Chairman

National Security Agency, Ft. Meade, Maryland 20755

Chairman's Introduction-9:00

\section{Contributed Papers}

\section{9:05}

CC1. Mathematical models of glottal area and vocal fold contact area. Ingo R. Titze (Department of Speech Pathology and Audiology, University of Iowa, Iowa City, IA 52242)

The glottal area function and the vocal fold contact area are computed as a function of a dynamically changing glottal configuration. Parameters are prephonatory widths and amplitudes of vibration of the upper and lower margins of the vocal folds, and the phase angle between the two. The computed waveforms are compared with measured photoglottographic (PGG) and electroglottographic (EGG) waveforms. The basic PGG and EGG waveform shapes are confirmed by modeling, although a simple relationship between vocal fold contact area and EGG may not always exist. It is demonstrated that symmetries in these glottographic waveforms are related to the shape of the medial surface contour of the vocal fold and should not be confused with asymmetries in the glottal volume flow [Rothenberg, Vocal Fold Physiology, edited by Stevens and Hirano (1981), pp. 305-323].

\section{$9: 20$}

CC2. Letter-to-phoneme rules: A semi-automatic discovery procedure. Dennis H. Klatt and David W. Shipman (Room 36-523, Massachusetts Institute of Technology, Cambridge, MA 02139)

A 20000 -word phonemic dictionary has been compiled from several sources and hand-edited so as to contain phonemic representations that are ideal input for the Klattalk synthesis-by-rule program or for an automated phonetic alignment system such as is planned for the Spire phonetics work station. The phonemic representation consists of 40 phonemes, primary and secondary stress symbols, and morpheme-structure symbols to indicate a prefix, suffix, or compound. Computer programs were then written to create letter-to-sound rules of the form $A \rightarrow[b] / C D$ EF, i.e., the letter " $A$ " goes to the phoneme [b] in the letter environment consisting of left context $C D$ and right context EF. Each instance of the letter " $A$ " in the dictionary creates a rule that may be new, the same as an old rule, or conflicting with an old rule (a letter goes to a different phoneme in the same context). Conflicting rules (which limit the performance of this procedure) are inevitable unless each whole word is stored in the left/right context. Conflicting rules arise because English spelling does not indicate where morpheme boundaries are located, which syllables are stressed, or the fact that the present work has undergone exceptional historical sound change or has been borrowed from another language. If only the most popular of each set of conflicting rules is used, the computer program can form the rules into a tree for rapid execution. The accuracy and tree size of this approach have been investigated as a function of (a) length of the left context, (b), length of the right context, (c) presence or absence of stress symbols in the orthography (which would require stress prediction from text, a problem that we have not studied as yet), and (d) presence or absence of morpheme boundary structure in the orthography. [Research supported in part by an NIH grant.]

\section{9:35}

CC3. How important is syntactic resolution of orthographic ambiguity? William M. Fisher (Texas Instruments, Inc., P.O. Box 226015, MS 238, Dallas, TX 75266)
Low-cost English text-to-speech systems typically do not use syntactic analysis. As a result, they must sometimes mispronounce certain words whose correct pronunciation depends on their surface syntactic category, such as "use." Using the grammatically tagged Brown Corpus as a model of English text, I estimated how much knowing the part-of-speech of words can help text-to-speech systems: the difference between the minimum phonemic error rate achievable with and without such syntactic knowledge is $0.015 \%$ counting only segmental errors, or $0.025 \%$ if stress errors are also counted. Six types of ambiguity were found: consonant voicing ("use"); location of primary stress ("present"); degree of secondary stress, with associated vowel reduction ("separate"); vowel syncope ("ragged"); "French final e" ("expose"); and vowel quality ("live"). Details of the calculations and lists of the words found to have these ambiguities will be presented.

\section{$9: 50$}

CC4. Position constraints on interacting segments in elicited errors favor the word over the stressed syllable. Stefanie Shattuck-Hufnagel (Research Laboratory of Electronics, MIT, Cambridge, MA 02139)

On the hypothesis that the position constraint on segmental interactions in speech errors reflects some aspect of lexical access during speech production planning, we have analyzed corpora of both spontaneous and elicited errors to determine the unit over which the position constraint operates. In spontaneous errors, two interacting segments are likely to occupy similar positions in both their respective words and syllables. Moreover, since most of the interaction errors in the MIT error corpus occur in initial position in words with first-syllable stress, it is difficult to determine which unit (word or stressed syllable) is the most appropriate one over which to state the constraint. Results of error elicitation experiments suggest that the word is the appropriate unit, since, for example, $/ \mathrm{j}$ / and $/ \mathrm{d} /$ are more likely to interact in the tongue twister "July dog dock Gillette" (where $/ \mathrm{j} /$ and $/ \mathrm{d} /$ are both word-initial) than they are in the twister "legit dog dock largesse" (where $/ \mathrm{j} /$ and $/ d /$ are both prestressed). Implications for models of lexical access during production planning will be discussed.

\section{0:05}

CC5. Towards an expert system for automatic stop consonant identification. Gary E. Kopec, Marcia A. Bush (Fairchild Camera and Instrument Corporation, 4001 Miranda Ave., Palo Alto, CA 94304), and Victor W. Zue (Research Laboratory of Electronics, MIT, Cambridge, MA 02139)

The adequacies of three sets of acoustic measurements for stop consonant recognition were assessed by examining the performance of a trained human spectrogram reader in a stop identification task. The data sets consisted principally of (1) gray-level pictures (wideband spectrograms); (2) two-dimensional line drawings (LPC spectra and short-time energy contours); and (3) tables of numerical measurements (e.g., frequencies, amplitudes, and bandwidths of spectral peaks). The reader's task was to identify the initial consonant in $/ \mathrm{CVb} /$ syllables selected from a corpus containing all combinations of the six stop consonants $/ \mathrm{b}, \mathrm{d}, \mathrm{g}, \mathrm{p}, \mathrm{t}, \mathrm{k} /$ with the six vowels $/ i, e, a e, a, o, u /$ as spoken by three male and three female talkers. The objective of the experiment was to identify an appropriate set 
of measurements for use in a knowledge-based expert system for automatic stop consonant identification. Results of the experiment will be presented and discussed in the context of this objective.

\section{$10: 20$}

CC6. Computation of vocal tract sensitivity functions: A complex Lagrangian approach. Francis Charpentier (Dept. RCP, CNET, 22301 Lannion, France)

A method for computing vocal tract sensitivity functions is presented in this paper. Fant and Pauli [Speech Commun. Semin., Stockolm, 121132 (1974)] have derived closed-form expressions relating the kinetic and potential energies in the tract to the sensitivity of formant frequencies with respect to small variations of the area function. In our approach, more general closed-form formulas are derived to compute the sensitivity of any quantity related to the vocal tract transfer function. A Lagrangian formulation allows a straightforward computation of the gradient of acoustic parameters with respect to area function parameters. This technique was used to replace the finite-difference gradient approximation in a procedure identifying articulatory parameters from formant parameters [Charpentier, ICASSP Proc., 1984-1987 (1982)]. The method takes into account the vocal tract losses (heat conduction, viscosity, wall vibrations, lip radiation). It was found effective for determining the articulatory parameters from outputs of the model itself.

\author{
Session DD. Plenary Session \\ David T. Blackstock, Chairman \\ President, Acoustical Society of America
}

Presentation of Awards

Wallace Clement Sabine Medal to Thomas David Northwood

Silver Medal in Engineering Acoustics to Per Vilhelm Brüel

Pioneers in Underwater Acoustics Medal to Arthur Olney Williams, Jr.

\title{
Lecture
}

Acoustic Microscopy. Calvin F. Quate (Department of Applied Physics, Stanford University, Stanford, CA 94305)

\section{Meeting of Standards Committee S2: Mechanical Shock and Vibration}

to be held jointly with the

\section{Technical Advisory Group (TAG) Meeting for ISO/TC 108 Mechanical Shock and Vibration}

\author{
P. H. Maedel, Jr., Chairman S2
}

Westinghouse Electric Corporation, Lester Branch, P.O. Box 9175, Lester, Pennsylvania 19113

G. Booth, Chairman Technical Advisory Group for ISO/TC 108

220 Clark Avenue, Branford, Connecticut 06405

\begin{abstract}
Standards Committee S2 on Mechanical Shock and Vibration. Working group chairpersons will present reports of their recent progress on writing and processing letter ballots on various shock and vibration standards. In addition, there will be a report on the interface of S2 activities with those of ISO/TC 108 (the Technical Advisory Group for ISO/TC 108 consists of members of S2, S3, and other persons not necessarily members of those committees). The Technical Advisory Group meeting will be held jointly with the meeting of Standards Committee $\mathbf{S} 2$.
\end{abstract}




\title{
Session EE. Education in Acoustics: Special Session on the Use of Computers in Acoustics Education
}

\author{
Lawrence A. Crum, Chairman \\ Department of Physics, University of Mississippi, University, Mississippi 38677
}

Chairman's Introduction $-8: 30$

Invited Papers

$8: 35$

EE1. Classroom exercise programs for numerically predicting and graphically displaying propagation characteristics of sound. Joseph A. Clark (Mechanical Engineering Department, Catholic University of America, Washington, DC 20064)

Several computer programs will be described which were developed for an introductory graduate course in underwater acoustics that was offered at a government laboratory (DTNSRDC) where Tektronix or H.P. graphics computers were available to most of the students. One program numerically solves the acoustic field equations in one dimension by a finite difference method. This program has been used to illustrate the reflection of sound at boundaries and effects of transmission into a second (or third) medium where the acoustic impedance and sound speed might differ. A second program numerically solves the acoustic field equations in two spatial dimensions. A ray tracing program will be described which illustrates the major features of sound propagation in inhomogeneous media and a program which plots beam patterns for an array with an arbitrary number of elements spaced at arbitrary distances apart will also be presented. The programs are written in BASIC and include interactive features which allow the students to explore a variety of propagation and radiation conditions on their own. The programs were found to provide a third useful way to develop a physical intuition for sound propagation characterstics when they were used in conjunction with studies of approximate analytical solutions of the field equations and laboratory demonstrations.

9:05

EE2. A Fourier-synthesis system with audible and visible ouput. W. James Hadden, Jr. (JHAD Acoustics, 2400 Westover Rd., Austin, TX 78703)

All too often, when teaching Fourier analysis in vibrations and acousties courses, one encounters a spurious resistance (from students) partially attributable to the absence of illustrations with tangible results. This paper describes the use of a commercially available digital/analog converter and microcomputer to provide demonstrations of Fourier series in which the usual time- and frequency-domain representations are supplemented by an audible output. Several versions of the driving software will be discussed. In one, the user may alter amplitudes of spectral components "on the fly" and soon thereafter hear the change in the acoustic signal while choosing between graphic displays of waveform or Fourier spectrum. In another, the user may enter spectral amplitudes and phases or fill in parameters in formulas for Fourier series-this information is then processed and stored for display as in the previous case. Convergence of Fourier sums to simple periodic functions, effects of relative phase changes, and responses of resonant systems to periodic excitation are among the topics that can be illustrated using this system. A brief demonstration is planned.

\section{9:35}

EE3. Some experiments with broadband ultrasonic pulses. M. Paul Hagelberg (Wittenberg University, P.O. Box 720, Springfield, $\mathrm{OH} 45501$ )

Several broadband ultrasonic pulse experiments will be presented which are suitable for use in the undergraduate laboratories. A brief description will be given of a set which requires only time domain measurements and for which the analysis is conceptually easy. Attention will be concentrated on a group which requires frequency domain analysis and for which digital data acquisition techniques are particularly suited. While these may be performed with relatively unsophisticated equipment they are readily adapted for microcomputer control and analysis.

\section{0:05}

EE4. Computer based laboratory instruction in acoustics and noise control. David K. Holger (Department of Engineering Science and Mechanics, 214 ERI Bldg., Iowa State University, Ames, IA 50011)

A computer based instructional laboratory in acoustics and noise control is described. The semester length laboratory, which is currently being developed, is based upon analog to digital ( $A$ to $D$ ) conversion of trans- 
ducer signals followed by micro- and/or main frame-computer analysis of the digitized signals. The proposed laboratory is compared with traditional laboratories in acoustics which normally require a number of standalone instruments. Extensions of the microcomputer based laboratory using the "micro" as a "smart" terminal in a main frame time sharing system are also discussed. The progression of experiments in the laboratory evolves through these phases. First phase experiments are designed to familiarize the student with transducers, $A$ to $D$ conversion and simple, stand alone, micro-computer analysis. Second phase experiments involve multichannel data acquisition and more extensive micro-computer analysis. Third phase experiments involve main frame software and data storage using the micro-computer as a "smart" terminal in a time sharing system. A phase two experiment pertaining to acoustic parameters describing the impulsive response of a room will be described and discussed in detail.

\section{0:35}

EE5. The development of a local micro-computer network for an acoustics laboratory. J. E. Kerivan and C. I. Holmer (E-A-R Div., Cabot Corporation, 7911 Zionsville Rd., Indianapolis, IN 46268)

Local computer networks (LCN's) lend themselves as a cost-efficient, growth-oriented, powerful answer to the problem of real-time control and processing of acoustic signals in a laboratory. The availability of inexpensive scientific and engineering "off-the-shelf" software which can be structurally integrated in a shared resource environment permits the realization of large data-base management systems with micro-computers. The comparison of LCN alternatives, both hardware and software options, as it relates to specific acoustic measurement problems will be contrasted in showing E-A-R's solution for a mini/micro-computer network. Modal analysis, reverberation-time measurements, damping data reduction, hearing protection attenuation, and analytical modeling are some of the tasks which are integrated in the system. In this presentation we will discuss in detail some of the alternatives considered to date in our system development and their perceived advantages and limitations. Listings of structured programs currently under development will be provided.

\section{1:05}

EE6. Computers in acoustics education at Lehigh. John B. Ochs (Lehigh University, 267 Packard Lab. 19, Bethlehem, PA 18015)

In the late 1970's the Industrial Engineering Department and the Mechanical Engineering Department of Lehigh University joined forces to develop and promote an integrated approach to the progression from mechanical design to manufacturing with computer graphics systems providing the man/machine interface. The primary focus of the CAD/CAM program is the education of the approximately 600 undergraduates in the two departments with the emphasis on the design and manufacturability of products so that an engineer can understand the ramifications of his design decision in an integrated CAD/CAM environment. The aim of the program is to provide state-of-the-art computer graphics systems as tools to improve design and manufacturing innovation. Educationally the goal is to implement the computer graphics technology into every undergraduate course in the two departments, which include such diverse topics as statics/dynamics, thermodynamics, mechanical vibration, statistics, operation research as well as the classical design and manufacturing. Some courses stress extensive use of these computer systems and the means to manage them, while others use the facilities for projects or homework assignments. By hard wiring the CAD and CAM laboratories to classrooms, the color real-time dynamic displays can be used as dynamic blackboards to visualize complex three-dimensional geometry or to illustrate multi-dimensional mathematical relationships. Growing from a strong undergraduate base the program has attracted graduate research from several engineering disciplines supported mainly by Lehigh's industrial partners.

\section{1:35}

EE7. Approaches for digital processing acoustic and vibration data in a teaching laboratory. Wolfgang Sachse, R. Cochran, S. Yu, C. P. Chen, and C. Chang (Department of Theoretical and Applied Mechanics, Cornell University, Ithaca, NY 14853)

In this paper we describe the signal acquisition and processing approaches we have implemented using a portable mini-computer system in laboratory experiments of acoustics and vibrations courses. Also described will be examples of programs we have developed to data which include the simulation of 1- and 2-degree-offreedom vibrating systems, the analysis of displacement, velocity, or acceleration signals of such systems; the digital processing of speech and other transient acoustic signals to obtain spectrograms; the determination of reverberation time; two-dimensional ray tracing in homogeneous and inhomogeneous layered media; and a general data acquisition, processing, and display program in which any one of more than 70 commands can be called with a three-letter mnemonic. Key to all the examples is the versatility of the system and the ease with which new experiments or examples can be implemented by inexperienced users. 


\author{
Session FF. Noise V: Measurement and Human Effects of Impulse Noise in the Workplace \\ John J. Earshen, Chairman
}

Metrosonics, Inc., P.O. Box 23075, Rochester, New York 14692

Chairman's Introduction-8:30

Invited Papeçs:

8:35

FF1. Impulsthoise measurement: The physiological basis. G. Richard Price (U.S. Army Human Engineering Laboratory, Aberdeen Proving Ground, MD 21005)

In designing a system to measure noise there are many interacting considerations, among them the electrical/acoustical state-of-the-art, regulatory requirements, administrative concerns, and economic issues. In contrast to these fluctuant elements, the structure of the auditory system is for all practical purposes fixed and establishes limits to many technical issues. Although theoretical work in the noise effects area is in its infancy, this paper will discuss the developing information with respect to the issues inherent in the measurement of noise, such as high- and low-frequency cutoffs, temporal factors, "trading ratios," and limits on the dynamic range.

9:05

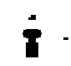

FF2. Intermittence and the total-energy hypothesis. W. D. Ward, C. W. Turner, and D. A. Fabry (2630 University Ave. \$E, Minneapolis, MN 55414)

The theory that auditory damage is proportional to the total A-weighted acoustic energy that has entered the ear has been shown to hold for single uninterrupted exposures to steady $700-2800-\mathrm{Hz}$ noise in the chinchilla, provided that a critical level of 110-115 dBA is not exceeded. The theory is not correct, however, if any appreciable interruptions in exposure occur. Earlier research [W. D. Ward, T. Kiester, and C. W. Turner, J. Acoust. Soc. Am. Suppl. 166 , S61 (1975)] had established that breaking up a 220-min exposure at 114 dB SPL into 22 10-min exposures, two per week, reduced both permanent threshold shift (PTS) and cochlear damage (missing outer hair cells, MOHC) by an amount equivalent to a reduction of at least $7 \mathrm{~dB}$ in level. Present results primarily compare the damage produced by a 15-day continuous exposure at $92 \mathrm{~dB}$ SPL to that geperated by nine weeks of "work-week" exposure (8 h/day Monday through Friday). The average PTS at 1, 2, 47/4d $8 \mathrm{kHz}$ was $11 \mathrm{~dB}$ for the continuous exposure, $4 \mathrm{~dB}$ for the work-week exposure, and the MOHC count dropped from 640 to 340 . The work-week values were similar to those observed in a group exposed continuously for 15 days to

$\mp \quad 85 \mathrm{dBSPL}$, indicating again a "saving" cquivalent to a 7-dB reduction, though this value is surely coincidental. Implications for exposure criteria will be discussed. [Work supported by NIH grant NS 12125.]

$$
\text { 9:35 }
$$

FF3. Industrial impact noise: Description and definition. John Erdreich, Thomas Doyle, and Steve Spaeth (National Institute for Occupational Safety and Health, Cincinnati, OH 45226)

In order to define the practical requirements of instrumentation for the measurement of industrial impact noise and to determine common parameters of industrial impact noise which should be considered in the study of the biological effects of such noise, survey measurements were made in industries which produce impulse noise. The measurements were made to include a bandwidth from $10 \mathrm{~Hz}$ to $40 \mathrm{kHz}$ and a dynamic range of either $\mathbf{4 2}$ or $72 \mathrm{~dB}$, for analog and direet digital recording, respectively. From the impact noise samples, we calculafed true rms sound pressure level for the burst, peak-level, crest factor based on burst duration, and 1-s equivalent continuous level. Dose based on 3- $\mathrm{dB} /$ doubling and 5-dB/doubling $\mathrm{A}$-weighted slow response and A-weighted fast response was calculated also. We will discuss the descriptors of impuse noise and also present preliminary data which address the variability of impulse noise signatures from the same process and the same machine.

\section{H.}

$$
\begin{gathered}
\text { 10:05 to 10:15 } \\
\text { Recess }
\end{gathered}
$$

\title{
Contributed Papers
}

\section{0:15}

FF4. The influence of continuous background noise on impact noiseinduced TTS. Raymond Hétu and Réjean Lazure (Université de Montréal, 2375, chemin côte Ste-Catherine, Montréal, Québec, Canada, H3T 1A8)
The present study assessed the influence of a cờtinuous background noise on the effect of the peak level and the number of impacts producing a $15 \mathrm{~dB} \mathrm{TTS}_{3}$ at 4, 6, and $8 \mathrm{kHz}$. A group of 15 normal-hearing adults were exposed to different conditions of noise. The critical peak level (CL) of an 
impact noise was first determined. The $C L$ defined as the level producing the criterion amount of $\mathrm{TrS}_{3}$ when the impacts were presented in series of 60 at a rate of one every $2 \mathrm{~s}$. This $C L$ was not altered by the introduction of a continuous broadband noise at $85 \mathrm{dBA}$. But it was lowered by 3 to $6 \mathrm{~dB}$ among one-third of the subjects when the background noise was at 105 dBA. Referring to the latter condition, both the peak and the continuous level were lowered by $4 \mathrm{~dB}$ and the number of impacts increased by a

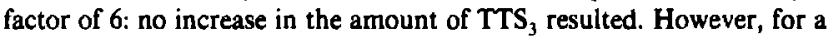
tenfold increase in the number of impacts, a 13-dB decrease in the peak and the continuous level did produce a slightly but significantly higher amount of $\mathrm{TTS}_{3}$. Implications of these results are discussed in term of exposure limits to industrial noise. [Work supported by INRS grant \# N/ D 25-80-25.]

\section{0:45}

FF5. Measurement of short duration high level impact noises. Per V. Brüel (Brüel \& Kjær, Nærum, Denmark)

Over the past few years there has been a growing suspicion that in shipyards, and in metal working industries with punch presses and forging processes, etc., the acoustic environment contains very short duration high level impulses. Since impulsive sounds as a rule are of very short duration, the human ear, and the standardized sound level meter can far from measure correctly the maximum sound pressures of impulses, simply because the integration time of the human ear and of the sound level meter is considerably longer than the impulse duration. Furthermore, the relatively long time intervals between short impulses, make the use of a sound level meter with "peak" holding capacity difficult in practice. In this article it is shown how the peak pressures can be measured three times a second and $L_{\mathrm{eq}}$ every minute with the aid of two sound level meters (one with $30-\mu$ s rise time and the other an integrating sound level meter) and a two channel level recorder. The instrumentation setup is battery driven and can operate without attention for seven days. The acoustic environments of a number of different industries have been measured with this set of instruments. The results show that in metal industries the levels of the short duration impulses are considerably higher than those found in wood industries.

\section{1:15}

FF6. Characterization and calibration of microphone systems for measurement of impulselike sounds. Victor Nedzelnitsky (National Bureau of Standards, Building 233, Washington, DC 20234)

Measuring the peak value of impulse noise is required by damage-risk criteria for hearing, material design standards, and for characterization of exposure to hazardous sound levels in industrial workplaces and the military. Other areas of application include building design and land-use planning. However, use of current proposed ANSI and IEC standards provides only highly accurate magnitude calibration of microphone cartridges and systems in response to sinusoids, and does not ensure accuracy in measuring the peak values of impulse noise. Convenient approximate methods for audio-frequency measurement include calibration of small, wideband transducers of ultrasonic resonance frequency by means of electrostatic actuators (for phase response and relative magnitude response) and by reciprocity-based methods (for absolute magnitude response at selected frequencies). Such transducer systems can have a fairly flat magnitude response and an approximately linear phase response throughout most of the audio range. Precision sources of transient or impulselike sound are also very useful. Several methods for realizing such sources are discussed; one particularly simple and robust device utilizes aerodynamic impact sound from colliding spheres. For in situ measurements of personal sound exposure, additional consideration must be given to the influences of microphone position and diffraction produced by the torso, head, and external ear.

\title{
Session GG. Physiological Acoustics IV: Response Activity from Central Auditory Sites
}

\author{
George M. Gerken, Chairman
}

Callier Center, Univeristy of Texas, 1966 Inwood Road, Dallas, Texas 75235

Chairman's Introduction $-8: 30$

\section{Contributed Papers}

\section{$8: 35$}

GG1. Alterations in the auditory brainstem response produced using a forward-masking paradigm. A. K. Ananthanarayan and George M. Gerken (Callier Center for Communication Disorders, University of Texas at Dallas, 1966 Inwood Road, Dallas, TX 75235)

The post-stimulatory effects of a masker on the probe-evoked auditory brainstem response (ABR) were evaluated in 18 normal-hearing subjects using a tone-on-tone forward-masking paradigm. The masker consisted of a $4 \mathrm{kHz}$ tone burst of $60 \mathrm{~ms}$ duration and the probe was a $4 \mathrm{kHz}$ tone burst of $10 \mathrm{~ms}$ duration. The probe was either centered in the masker or followed the masker by various offset-to-onset intervals $(\Delta t)$. Stimulus intensity was specified for each subject re: threshold for the masker. The latency versus $\Delta t$ functions for waves $I I I$ and $V$ and the amplitude versus $\Delta t$ function for wave III were compatible with a partial forward-masking interpretation. However, the wave $\mathrm{V}$ amplitude versus $\Delta t$ function showed amplitude increments re: the unmasked wave $V$ amplitude for some values of $\Delta t$ (enhancement). Other stimulus variables manipulated included the masker frequency, the probe frequency, the relative intensity of the masker, the absolute intensity of the probe and masker, and the rise/ fall times of the masker, Enhancement of wave $V$ is a robust phenomena obtainable with a broad but definite set of stimulus conditions.

\section{8:50}

GG2. Auditory nuclei in the brainstem exhibit hypersensitivity to electrical stimulation as a result of sound-induced hearing loss. George M. Gerken, Robin E. Paul, and Samuel S. Saunders (Callier Center for Communication Disorders, University of Texas at Dallas, 1966 Inwood Road, Dallas, TX 75235)

Thresholds for the detection of electrical stimulation applied to auditory brainstem nuclei were previously demonstrated to decrease by 6 to 15 $\mathrm{dB}$ in most cases following mechanical destruction of the cochleas. In the present work electrodes were located in cochlear nucleus and in the nucleus of inferior colliculus, and detection thresholds for electrical stimulation were measured behaviorally. A 48-h exposure to a $1-\mathrm{kHz}$ sine wave of approximately $110 \mathrm{~dB}$ SPL resulted in temporary threshold shifts of $50 \mathrm{~dB}$ 
or more and permanent threshold shifts of 10 to $30 \mathrm{~dB}$ as evaluated using evoked potential techniques. Behaviorally measured electrical-stimulation thresholds were obtained for each electrode for a number of sessions prior to and following noise exposure. Stimulation thresholds decreased by 2 to $17 \mathrm{~dB}$ (hypersensitivity) immediately following the exposure to sound and for most electrodes this hypersensitivity was maintained even after recovery from the temporary portion of the threshold shift. These results indicate that a moderate hearing loss may alter the condition of central auditory mechanisms. [Work supported by NIH Grant NS 16411.]

\section{9:05}

GG3. An automatic electric response audiometer, Robert Galambos and Scott Makeig (SHNSC, Childrens Hospital, 8001 Frost Street, San Diego, CA 92123)

Available evoked potential procedures vary widely in how fast and how accurately they assess sensitivity at the audiometric frequencies (250 through $4000 \mathrm{~Hz}$ ). We will describe a device that can extract this information quickly and automatically. As in Bekesy audiometry, it uses patient responses to control attenuator settings which are then plotted as a function of time. The responses used, however, are the sine-wave like brain potentials recorded at the scalp during monaural presentation of tone pips at a rate around $40 \mathrm{~Hz}$ [Galambos et al., Proc. Natl. Acad. Sci. USA 78, 2643 (1981)]. Cross correlating several hundreds of these with a sinusoidal template yields a number which, depending on whether it is high or low, causes a $5 \mathrm{~dB}$ decrease or increase, respectively, in the sound intensity. The procedure is then repeated at the new sound level. A computer processes the responses, performs the statistical computations and resets the attenuator every 5-15 s. A threshold estimate can be obtained in a few minutes; these agree well with the behavioral for alert adults, but when the mind wanders, and during drowsiness and sleep, they can rise by $20-30$ dB.

\section{$9: 20$}

GG4. Effects of stimulus presentation rate on the middle-latency auditory evoked potentials. David $R$. Stapells (Department of Psychology, University of Ottawa, Ottawa, Canada K1N 6N5), J. Braxton Suffield (Department of Psychology, Carlton University), and Terence W. Picton (Department of Medicine, University of Ottawa, Ottawa, Canada K1N 6N5)

The middle-latency evoked potentials were recorded in response to 60 $\mathrm{dB}$ nHL $500 \mathrm{~Hz}$ tones with rise and fall times of $4 \mathrm{~ms}$ and plateau durations of $2 \mathrm{~ms}$. The tones were presented at rates of between 10 and $60 / \mathrm{s}$ using 5/s steps. Replicate averages of 2000 evoked potentials each were analyzed using a sweep of $50 \mathrm{~ms}$ in ten subjects. The response had a roughly sinusoidal waveform and was repeated twice in the $50 \mathrm{~ms}$ period. The size of the response was measured as the average of the peak-to-peak amplitudes in the $50 \mathrm{~ms}$ sweep. The amplitude was greatest at stimulus rates of $40(1.12 \mu \mathrm{V})$ and $45 / \mathrm{s}(1.15 \mu \mathrm{V})$. It was about half this amplitude (between 0.54 and $0.75 \mu \mathrm{V}$ ) at rates of less than $35 / \mathrm{s}$ or greater than $50 / \mathrm{s}$. The morphology of the response and its relation to stimulus rate were quite similar from one subject to another. These results extend and replicate those of Galambos et al. [Proc. Natl. Acad. Sci. USA 78, 2643-2647 (1981)]. They indicate a steady-state auditory evoked potential with a resonant frequency of $40-45 \mathrm{~Hz}$

\section{$9: 35$}

GG5. The effects of within-train intensity changes on wave $V$ adaptation. Bob Burkard and Kurt Hecox (Waisman Center, University of Wisconsin-Madison, Màdison, WI 53706)

Adaptation of the BER was evaluated using stimulus trains containing one or two click intensities. Single-intensity trains consisted of eight clicks presented at 40,50 , and $60 \mathrm{~dB}$ nHL. For trains comprised of two click intensities, the first four clicks were presented at $50 \mathrm{~dB} \mathrm{nHL}$ and the last four clicks at 40 or $60 \mathrm{~dB}$ nHL. In the two-intensity trains, the responses to the fifth click (the first response following the intensity shift) were significantly delayed compared to the first click of the 40 and $60 \mathrm{~dB}$ nHL control series. For all click trains, wave $V$ latency shifts had asymptoted by click 5 , demonstrating that wave $V$ adaptation is not intensity specific. The magnitude of latency shift by the fifth stimulus in the train showed some dependency on the intensity of the initial clicks. We can tentatively conclude: (1) intensity shifts of $10 \mathrm{~dB}$ do not interrupt the adaptation process, (2) asymptotic latency shift is attained by the tifth click in the train, even if click intensity is changed mid-train, and (3) under some conditions the magnitude of latency shift may be dependent on the relative intensity of the initial and terminal clicks in the train. [Work supported by NIH.]

\section{9:50}

GG6. BER adaptation paradigm: Stimulus dependencies. Lynn Weatherby and Kurt Hecox (Waisman Center, University of Wisconsin, Madison, WI 53706)

The time course of wave $V$ latency shifts (adaptation paradigm) to a train of eight noise bursts with variable duration, rise-fall time, and intensity was investigated. Wave $V$ latency increased as a function of increasing position in the train for all conditions. Approximately $70 \%$ of the total latency shift occurred within the first four stimuli of the train. Unlike click stimuli the asymptotic latency was not attained by the eighth stimulus in the train. Rise-fall time $(0,1,2, \mathrm{~ms})$ increased the latency of wave $V$ to the first stimulus in the train but the magnitude and rate of adaptation were not affected. Increasing stimulus duration $(2,5,7 \mathrm{~ms})$ did not affect the latency of the first response in the train but did affect the magnitude and rate of adaptation. Increasing intensity increased the latency to the first stimulus and the magnitude of adaptation. These stimulus dependencies suggest that forward masking contributes to the latency shifts observed in the adaptation paradigm. [This work is supported by NIH.]

\section{0;05}

GG7. Steady-state evoked potentials in humans to continuous amplitude modulated tones. Field W. Rickards and Graeme M. Clark (Department of Otolaryngology, University of Melbourne, Parkville, Australia 3052)

Phase locking a computer to the modulation envelope of a continuous sinusoidally modulated amplitude modulated tone, enables an averaging technique to be used to record a periodic, steady-state evoked potential from the scalp of normal human subjects. The periodic evoked potentials had the same period as the modulation waveform. Fourier analysis was used to quantify the amplitude and phase of the first and second harmonics of the response. Responses were recorded for modulation rates from 4 to $448 \mathrm{~Hz}$, for carrier frequencies from $250 \mathrm{~Hz}$ to $4 \mathrm{kHz}$ and for sound pressure levels from 30 to $100 \mathrm{~dB}$ SPL. In general, the response amplitude increases with SPL. Estimates of latencies of these steady-state evoked potentials can be made by measuring the phase change of both harmonics as the modulation frequency is varied. The latencies appeared to have one of 14 discrete values from $3 \mathrm{~ms}$ up to $104 \mathrm{~ms}$ depending on the stimulus parameters. The majority of the responses had latencies from 9 to $33 \mathrm{~ms}$.

\section{0:20}

GG8. Asymmetries in the auditory brainstem response: Estimates of reliability and variance. C. Berlin, P. Allen, and L. Hughes (Department of Otorhinolaryngology and Biocommunication, Kresge Hearing Research Laboratory, Louisiana State University, 1100 Florida Avenue, New Orleans, LA 70119)

At the previous meeting of the society we presented data on binaural asymmetries in the auditory brainstem response. Individual data show that the binaural interaction was difficult to replicate and resisted systematic reliability studies. In the present studies a factorial design was used to reduce contribution of earphone and electronic channel assymmetries. Although binaural interaction (defined as the sum of binaurals minus the sum of monaurals [ $\Sigma$ BIN- $(\Sigma$ MON $R+L)]\}$ was seen in group data it was not reliably obtained from all subjects under all conditions, confirming R. Dobie [J. Acoust. Soc. Am. 71, 1031-1033 (1982)]. However, differences between responses acquired from the right and left sides of the head were quite systematic, and reliably duplicable from subject to subject and from condition to condition. A statistical program to perform point-bypoint analysis of each of the auditory brainstem responses was written and designed by one of us (LFH) and allows an exact probability determination that specific similarities occur by change. [Supported by NINCDS \#NS 11647.1 
10:35

GG9. An afterhyperpolarization model for vestibular afferents. Charles E. Smith (Department of Biometry, MUSC, Charleston, SC 29425) and Jay M. Goldberg (Department of Pharmacological and Physiological Sciences, University of Chicago, Chicago, IL 60637)

A stochastic afterhyperpolarization model, modified from Kernell (Brain Res. 11, 685-687 (1968)], simulates many features of the steadystate discharge of vestibular afferents, and may be relevant for spike initiation mechanisms in auditory afferents. Major assumptions are: (a) a single trigger site and firing occurs when the transmembrane voltage reaches a fixed critical level; (b) postsynaptic voltage fluctuations are responsible for interspike-interval variability and are due to random times of occurrence of quantal synaptic inputs; (c) postpike recovery is due to a time-dependent decrease in the potassium conductance $\left(\boldsymbol{g}_{k}\right)$, i.e., an afterhyperpolarization; (d) cumulative after hyperpolarization is responsible for the lengthening of the mean interval following an interposed spike; and (e) regular and irregular afferents differ only in the magnitudes of their afterhyperpolarization. The model predicts a stronger dependence of galvanic sensitivity on normalized coefficient of variation of interspike intervals than is actually observed. The discrepancy can be explained by assuming a fourfold variation in the size of synaptic quanta characterizing different vestibular afferents. [Work supported by NSF, S.C. Appropriation for Biomedical Research.]

THURSDAY MORNING, 11 NOVEMBER 1982

INDIAN RIVER ROOM, 8:30 TO 12:05 P.M.

\title{
Session HH. Shock and Vibration III: Shock and Vibration Standards and Fluid Related Vibration Studies
}

\author{
Paul H. Maedel, Jr., Chairman \\ Steam Turbine-Generator Division, Westinghouse Electric Corporation, Lester P.O. Box 9175, Philadelphia, Pennsylvania \\ 19113
}

Chairman's Introduction-8:30

Invited Papers

8:35

HH1. Machinery vibration standards. Jack B. Catlin (IRD Mechanalysis, Inc., 6150 Huntley Road, Columbus, OH 43229) and Paul H. Maedel, Jr. (Westinghouse Electric Corporation, P.O. Box 9175, Philadelphia, PA 19113)

Over the past 20 years the use of vibration measurement has grown rapidly in the areas of machinery mechanical condition monitoring and evaluation. As a result of this growth, a number of measurement techniques and measurement parameters have evolved. While there has been some commonality in these techniques and parameters, it has often been found difficult to compare machine condition when the same techniques and parameters were not used. Other associated problems have also resulted. This variability in techniques and parameters indicated a real need for vibration measurement standardization within the United States, and throughout the world. To meet this need, standardization committees were formed by the American National Standards Institute (ANSI) to handle U.S. standardization requirements, and by the International Standards Organization (ISO) to produce vibration standards acceptable on a worldwide basis. Vibration Standards offer a potential of a number of advantages: (1) machinery purchase specification simplification, (2) machine design guidelines, (3) machine evaluation criteria, (4) a more useable data base, and (5) easier interchange of technical information. As a result of these advantages, a number of national (ANSI) and international (ISO) machinery vibration standards have been developed. This paper reviews those machinery vibaration standards which have been published, as well as those which are in-process and planned.

\section{9:05}

HH2. Development of standards on vibration effects on buildings. G. R. Coonan (Peabody Coal Company, St Louis, MO 63178), D. E. Siskind (U.S. Bureau of Mines, Twin Cities Mining Research Center, Minneapolis, MN 55417), and S. P. Ying (Gilbert/Commonwealth, Jackson, MI 49201)

One of the Working Groups of the American National Standards Institute S2 Committee on Mechanical Shock and Vibration is \$2-78 on Measurement and Evaluation of Structural Vibration. This working Group is currently participating in the development of a standard for building vibration with the corresponding Working Group of the International Standards Organization TC 108/SC2/WG3. Consensus standards of this type provide technical guidelines to the Government agencies responsible for establishing regulations for environmental conditions as well as occupational health and safety. The proposed standards on building vibration specify the basic principles of performing vibration measurement and processing data, in regard to using the results of measurements for evaluating vibration effects on buildings. The evaluation is primarily directed toward structural response, but appropriate analytical methods are also included. The response as affected by vibration frequency, duration, and amplitudes as induced by any source is examined. The status of the proposed international standards and the available empirical data on damage from various sources are reviewed. 
HH3. Vehicle vibration standards: From the ground up. J. C. Barton (Caterpillar Tractor Company, 100 Northeast Adams, Peoria, IL 61629)

ANSI S2-67 includes the measurement and evaluation of mechanical vibration and shock in land vehicles. The starting point of the work is the ground, or roadway, as a principal source of vehicle vibration. The end point is vibration and shock data relevant to vehicle or component service life, and/or to the occupants of the vehicle. The standards work of ANSI S2-67 ranges from test instrumentation, through test methods and data analysis, to the form of the final report. Through its liaisons with other USA standards organizations, ANSI S2. 67 coordinates the USA position on ISO proposals for standards and provides the USA input to the corresponding 1 SO working group. Hence, ANSI S2-67 insures that USA views are represented in the development of international standards. The international standards have as their objective the removal of technical barriers to trade and the facilitation of international trade, hence are important for the export of USA products and are beneficial to the USA economy.

\section{Contributed Papers}

\section{$10: 05$}

HH4. Full-scale and model acoustic experiments on a ro-ro container ship. Alex de Bruijn (Institute of Applied Physics, TNO-TH (TPD), P.O. Box 155, 2600 AD Delft, The Netherlands)

To obtain a systematic set of data on noise and vibration due to propeller cavitation a comprehensive program of model and full-scale hydrodynamic and acoustic investigations was carried out during the years 19791981 on a single screw 39000 dwt Dutch ro-ro containership (speed, power, full-scale cavitation viewing, noise, and vibrations simultaneously). This paper reviews some of the acoustic results in connection with the possible application of compliant layers on the shell plates in way of the propeller. A simplified acoustic scale I: 10 model of the stern part has been constructed in steel, including proper scantlings, bulkheads, rudder and skeg, and checked for reliability by means of an electrodynamic hydrosounder located at the propeller disk. The model and full-scale ratios of sound pressure in front of the plating to velocities of the shell and of some decks agreed satisfactorily except below $20 \mathrm{~Hz}$ (full scale). The acoustic effect of an a priori seaworthy compliant layer, investigated by means of the model, appeared satisfactory, although it was limited by the flanking paths through rudder and skeg. The model also showed that a useful reduction of the transmission of cavitation noise by an increase of the source-to-shell-distance can only be obtained for frequencies above $25 \mathrm{~Hz}$ (full scale).

\section{0:20}

HH5. Structureborne sound transmission in fluid filled piping system elbows. N. Duke Perreira (Department of Mechanical Engineering, Taylor Hall Mail Room, The University of Texas at Austin, Austin, TX 78712)

A newly developed technique to analyze structureborne sound transmission in fluid filled piping system elbows based on wave theory is described. It is believed that generalization of the technique can be used to analyze structureborne sound transmission in fluid filled nonplanar piping systems that include bends and branches. To illustrate some of the basic principles of the method, an example is included. The wave theory approach is commonly used in analyzing electromagnetic and acoustic wave transmission in thin waveguides. Use of the technique to analyze structural vibrations is not as common. Other methods used to analyze the dynamics of structures include the differential equation and energy methods. Comparison of the experimental results with that using computational techniques based on the various theories show that the wave theory leads to much more accurate results than the differential equation solution method. Both the differential and the wave theory are based on force and moment analysis using a free body diagram of a differential system element; thus the system responses based on the two theories should be equal. The differences in answers using the two techniques is due to computational and numerical errors. In the wave theory the form of the system deformation functions are assumed while in the differential equation method, the form of the solution is obtained numerically using a finite element method. The wave theory method is not without its own set of problems. The differential equation can include higher order affects such as rotary inertia and Poisson effects; the wave theory method requires that the deformation be written in terms of exponential functions or in terms of the sine, cosine, hyperbolic sine, and cosine functions. This latter requirement of the wave theory method may not allow the higher order affects to be easily included in the system model. This does not seem to be a problem in the analysis of piping systems where the higher order affects are probably not important.

\section{0:35}

HH6. Doubly asymptotic approximations for vibration analysis of submerged structures. Thomas L. Geers and Carlos A. Felippa (Lockheed Palo Alto Research Laboratory [52-33/255], 3251 Hanover Street, Palo Alto, CA 94304)

Doubly Asymptotic Approximations (DAA's) are differential equations for boundary-element analysis of the dynamic interaction between a complex structure and a surrounding infinite medium [T. L. Geers, J. Acoust. Soc. Am. 64, 1500-1508 (1978); P. G. Underwood and T. L. Geers, Int. J. Solids Struct. 17, 687-697 (1981)]. In this paper, the use of first- and second-order DAA's for vibration analysis of submerged structures is examined. First, the governing discrete-element equations for the general problem are set down and discussed. Then the accuracy of three DAA forms is studied through the generation of numerical results for a submerged spherical shell. Although the first-order DAA is found to be inadequate, the two second-order forms show considerable promise. [Work supported by the David W. Taylor Naval Ship R \& D Center.]

\section{0:50}

HH7. Resonant oscillations of fluid-loaded struts. D. G. Crighton (Department of Applied Mathematical Studies, University of Leeds, Leeds, England

A simple model is used to describe how radiation damping limits the resonant oscillations of a cantilever or strut. In the low-frequency limit, radiation damping is equivalent to a fictitious internal dissipation, with a loss factor $\eta_{\text {rad }}$ slightly higher than typical mechanical loss factors, showing that resonant response is limited only by radiation loss, even for thin struts for which $k_{0} a<1$. When $k_{0} a>1$ it is found that $\eta_{\mathrm{rad}}$ is much larger than any internal loss factor and therefore that high-frequency resonances are heavily damped, regardless of internal dissipation. The pressure field near the strut is also examined, and in the case $k_{0} a<1$ is found at resonance to exceed the driving pressure by the large factor $\left(k_{0} a\right)^{-2}$. The farfield pressure at resonance may, under some conditions, also greatly exceed the off-resonance scattered field, and thiš great scattering efficiency of a thin strut at resonance is demonstrated by detailed examination of a particular case.

\section{1:05}

HH8. Acoustic radiation from point-driven, doubly periodic ringsupported, fluid-loaded circular cylinders. C. B. Burroughs (Applied Research Laboratory, Pennsylvania State University, P.O. Box 30, State College, PA 168011

Closed form analytic expressions are derived for the farfield acoustic radiation from a point-driven, fluid-loaded circular eylindrical shell reinforced with doubly periodic ring supports. The ring supports interact with the cylindrical shell only through normal forces; moments for the supports are not included. The analytic expressions include the effects of fluid loading and interactions between supports. Characteristics of the acoustic 
radiation are examined by example and some physical interpretation of significant features are offered.

HH9. The entrapped elasticity of a fluid-loaded plate. Alan D. Stuart (Applied Research Laboratory, Pennsylvania State University, P.O. Box 30, State College, PA 16801

It is basic knowledge that the wave equation for an acoustic fluid represents a force balance between an inertia and an elastic-restoring component in much the same way a balance exists between the mass and spring forces of a simple oscillator. It is also commonly known that only the mass component contributes to sound radiation from a simple pulsating convex source like a pulsating sphere, cylinder, or circular piston in a baffle. (By simple source one usually means a source whose dimensions $a$, are small compared to the acoustic wavelength, that is $k a<1$.) This paper will explore the situations under which the elastic component of an acoustic field arise, and specifically, consider how both the mass and elastic components of the acoustic field contributes to the vibratory response of a fluid-loaded plate.

\section{1:35}

HH10. Effects of a rigid coupling on wave propagation in fluid-filled elastic cylinders. Robert T. Menton (Code 5844, Naval Research Laboratory, Washington, DC 20375)

In order to more closely approximate the true nature of pertinent experimental facilities, the effects of a short, fluid-filled rigid coupling on wave propagation into a coaxial, fluid-filled, semi-infinite elastic cylinder are modeled. A vibrating piston is located at the end of the rigid coupling that is remote from the elastic cylinder. Linear plane-wave propagation is assumed, with the rigid coupling supporting fluid-borne waves at the speed of sound in the fluid, and the elastic cylinder supporting hydroelastic breathing and extensional waves. Compared to the case where the vibrating piston is located right at the elastic cylinder (i.e., no coupling), it is found that the amplitude of breathing waves in the elastic cylinder is nearly doubled. In addition, breathing waves propagate down the cylinder as though they originated at a position slightly in front of the vibrating piston. These results are useful for correlating elastic cylinder modeling with experimental data.

$$
\text { 11:50 }
$$

HH11. Dispersion relation for composite waveguide with application to measurements in acoustic impedance tube. Pieter S. Dubbeiday and Rodger N. Capps (Naval Research Laboratory, Underwater Sound Reference Detachment, P.O. Box 8337, Orlando, FL 32856)

The amplitude and phase of the reflected wave in an acoustic impedance tube are related to the speed and attenuation of sound in the sample material. It is usually assumed without justification that the pertinent propagation speed is the dilatational speed. To investigate this an analysis was performed on the propagation of axially symmetric waves in a composite waveguide consisting of a solid cylinder immersed in a fluid bounded by a concentric rigid wall. The roots of the ensuing dispersion relation were computed for various materials. The results show that the sound speed in the waveguide may vary between the dilatational and the bar speed, depending on the size of the fluid gap and the elastic properties of solid and fluid. Experiments are designed to verify this effect by changing the sample radius and by excluding the fluid from the gap between sample and wall.

\title{
THURSDAY MORNING, 11 NOVEMBER 1982
}

BROWARD AND PALM BEACH ROOMS, 8:30 to 11:15 A.M.

\author{
Session II. Underwater Acoustics V: Propagation (Précis-Poster Session)
}

\author{
Halcyon E. Morris, Chairman \\ Code 300, Naval Ocean Research and Development Activity, NSTL Station, Mississippi 39529
}

\author{
Chairman's Introduction-8:30
}

\section{Contributed Papers}

\section{$8: 35$}

II1. A study of secondary sound channels due to temperature inversions in the Northeast Pacific Ocean. R. K. Chow and D. G. Browning (Defence Research Establishment Pacific, FMO, Victoria, B.C. V0S 1B0, Canada)

Above $45^{\circ} \mathrm{N}$ latitude the circulation of the Northeast Pacific Ocean is controlled by the counterclockwise Alaskan Gyre of subarctic water. Due to a greater freshwater influx than evaporation, the water column is characterized by the three distinct layers: $0-100 \mathrm{~m}$ depth, cold, low salinity water; $100-200 \mathrm{~m}$, zone of strong salinity gradient (halocline); $200 \mathrm{~m}$ and below, gradual changes in temperature and salinity. Roden [J. Geophys. Res. 69, 2899-2914 (1964)] has shown that the middle halocline layer is the site of a complex temperature structure with numerous temperature inversions. These temperature inversions result in the formation of secondary sound channels which our analysis shows are widely distributed. Using a PE prediction model the cutoff frequency of a typical secondary sound channel was determined to be approximately $80 \mathrm{~Hz}$. Since depth excess exists at most locations, energy not trapped in the secondary duct remains in the deep sound channel. Relative loss in the two channels is presented as a function of frequency $(25-200 \mathrm{~Hz})$ and range $(0-100 \mathrm{~km})$ for various source and receiver depths. Under optimum conditions the existence of a secondary sound channel can decrease the average propagation loss by $20 \mathrm{~dB}$.

112. Low-frequency attenuation in the North Pacific subarcticsubtropical transition zone. D. G. Browning and R. K. Chow (Defence Research Establishment Pacific, FMO, Victoria, B. C.VOS 1BO, Canada)

The water mass regions in the North Pacific are relatively well defined. Kibblewhite el al. [J. Acoust. Soc. Am. 61, 1169-1177 (1977)] have shown that each water mass has a characteristic low frequency (below 200 $\mathrm{Hz}$ ) attenuation. They find the highest attenuation to be in the SubarcticSubtropical Transition Zone and these values are the highest yet reported in any ocean for this frequency range. It has recently been reported by Focke et al. [J. Acoust. Soc. Am. 71, 1438-1444 (1982)] that such values of attenuation can be modeled using a frequency independent mechanism, exponentially decreasing from the surface, the origin of which is unspecified. We compare these results with attenuation coefficients we have obtained in this zone. The oceanographic and biological properties of this region are analyzed to determine possible scattering mechanisms and 
their distribution. The effect of the two frontal areas bounding this region on the determination of attenuation coefficients is also examined.

\section{8:43}

II3. More on replacing transitional acoustic ray fields by a bundle of modes. E. Niver, A. Kamel, and L. B. Felsen (Department of Electrical Engineering and Computer Science, Polytechnic Institute of New York, Farmingdale, NY 11735)

The failure of ray acoustics in transition regions near single and multiple caustics, bottom glancing and critically incident rays in a vertically stratified ocean can be repaired by replacing these ray fields with a bundle of guided modes plus truncation remainders. The theory for this equivalence is exact, and has been verified by direct numerical implementation of the various constituents [E. Niver, A. Kamel, and L. B. Felsen, J. Acoust. Soc. Am. Suppl. 171 S66 (1982)]. The study has now been extended by treating the remainder not only as a modified ("collective") ray field as presented previously [L. B. Felsen, J. Acoust. Soc. Am. 69, 352 (1981)] but alternatively as a modified (collective) modal field. Moreover, asymptotic considerations have been employed to simplify the computer program. Detailed numerical comparisons for a model profile reveal the accuracy and numerical efficiency of various mode and remainder options as applied to the transitional ray fields noted above. It is shown that modal replacement of rays near single or multiple caustics also accounts for non. negligible evanescant ray fields. Moreover, it is preferable to treat the remainders as a modified modal field instead of a modified ray field since the width of the equivalent mode bundle can be reduced thereby. [Work supported by ONR Ocean Acoustics Branch.]

\section{$8: 47$}

II4. A comparison of acoustic propagation predictions for surface duct environments using ray-theoretic and parabolic-equation computer codes. A. Tolstoy, E. R. Franchi, and K. R. Nicolas (Acoustics Division, Code 5160, Naval Research Laboratory, Washington, DC 20375)

An important type of under water acoustic environment involves a deep water SOFAR channel with a superimposed (winter) surface duct capable of trapping acoustic energy. In this paper we examine the effects predicted by a computer model of three ducted sound-speed profiles on acoustic propagation at $300 \mathrm{~Hz}$. The purpose of this study was to better understand the capabilities and limitations (both theoretical and numerical) of a ray theoretic model for predicting mid- and long-range signal transmission loss (TL) in the winter North Atlantic. Several source and receiver configurations (combinations with one or both in and out of the duct) have been studied. To test validity, the ray theoretic calculations are compared to previously accepted results predicted by a propagation model based upon the parabolic equation. It is found that both model predictions agree qualitatively (TL measurements and acoustic field patterns are comparable), while any quantitative discrepancies are explained by the theoretical or numerical limitations of the different approaches. We conclude that when both the source and receiver are at least several wavelengths from the sea surface, ray theory can be quite accurate in its prediction for these ducted environments. [Work supported by Naval Electronic Systems Command, Code 612.]

\section{8:51}

II5. Multipath propagation in a deep ocean including modified rays near grazing incidence at the bottom. Ronald F. Pannatoni (Bell Laboratories, Whippany, NJ 07981\}

A ray description of acoustic propagation between communicators submerged in a deep ocean may include rays that almost graze the interface between water and sea-floor sediment. For example, this can occur in range-independent environments where the squared refractive indices in water and in sediment decrease linearly with depth. Four pairs of such rays connect communicators near the surface and separated in range by at most $4(H / \gamma)^{1 / 2}$, where $H$ is the ocean depth and $\gamma$ is the gradient of square refractive index in water. One ray in each pair is entirely waterborne; the other intercepts the sediment and grazes a caustic. We present a uniform asymptotic analysis of these ray pairs that incorporates diffractive finite frequency effects as in the modified ray theory of Murphy and Davis. These effects include the appearance of a frequency-dependent caustic and a modification of the usual $\pi / 2$ phase shift experienced by the classical ray that intercepts the sediment. Comparison of these results with normal mode calculations is excellent. Vector diagrams (after Bartberger) also identify mode groups corresponding to the modified rays.

\section{8:55}

116. A formula for the ray density over a caustic surface for variable index media. Donald G. Burkhard (Department of Physics, University of Georgia, Athens, GA 30602)

The acoustic ray density becomes infinite over caustic surfaces and therefore cannot be used as a measure of the concentration of rays at or near the caustic. A finite substitute measure, the density of rays tangent of the caustic, may be obtained by dividing an element of incident flux by the area of the caustic formed by the associated rays. This provides a measure of the energy density over different regions of the caustic. As an example the ray density over the caustic is evaluated for a linear depth dependent index of refraction.

\section{8:59}

II7. Application of two-variable Taylor series to the ray theory of propagation in an unbounded medium. Marshall Hall ${ }^{a /}$ (Naval Ocean Systems Center, Code 712, San Diego, CA 92152)

The ensonified acoustic field near the initial caustic in the first convergence zone is considered. This field is formed by a pair of rays that are heading downward at the source and upward at the receiver. The ray parameter (namely the phase velocity) of each ray is expressed as a twovariable Taylor series in horizontal range and depth relative to a fixed expansion point. The terms of the series are calculated for derivatives up to fifth order. The method is applied to a typical oceanic example in which the caustic of the rays passes the expansion point at a vertical distance of $15 \mathrm{~m}$ and at a horizontal distance of $200 \mathrm{~m}$. The Taylor-series phase velocities are inserted into the usual ray theory expressions for the relative energy and travel time. It is found that satisfactory accuracy is obtained over a region of the ocean that is sufficiently large for several sonar applications. The advantage of this new approach is that, to calculate the sound field at a grid of points within a region, it is sufficient to determine (by iteration the ray parameters of only the two rays that pass through the expansion point. ${ }^{21}$ On exchange from the Defense Science and Technology Organization, P.O. Box 706, Darlinghurst, NSW 2010, Australia.

\section{9:03}

II8. Sound propagation in a surface duct: Can the deep water profile be neglected? Marshall Halla) (Naval Ocean Systems Center, Code 712, San Diego, CA 92152)

In calculating normal-mode acoustic propagation in an ocean surface duct, the sound-speed profile is often represented by the simple bilinear profile. The question arises as to whether the positive gradient in the sound-speed profile that occurs beneath the SOFAR axis has a significant effect on surface duct propagation. Calculations have been made for typical oceanic profiles and it has been found that the bilinear profile and the complete profile yield the same result as a function of range (even though the individual normal modes are different) until the convergence zone effect becomes significant. The threshold range at which this effect becomes significant decreases as frequency is decreased below the surface duct's cutoff frequency (since the energy propagated within the duct decreases while the width of the convergence zone increases). For a duct that has a cutoff frequency of $200 \mathrm{~Hz}$ for example, the threshold range decreases from 30 to $15 \mathrm{~km}$ as the frequency decreases from 200 to $100 \mathrm{~Hz}$. a) On exchange from the Defence Science and Technology Organization, P.O. Box 706, Darlinghurst NSW 2010, Australia.

\section{9:07}

119. Acoustic sensitivity to sound-speed profile selection in the deep ocean. P. Bilazarian, ,' W. L. Siegmann, and M. J. Jacobson (Rensselaer Polytechnic Institute, Troy, NY 12181)

The effect of the selection of a deep-ocean sound-speed profile on the sensitivity of acoustic receptions is examined. In our initial study [J. Acoust. Soc. Am. Suppl. 69, S34 (1981)], the fixed sound source and receiver were positioned on the ocean boundaries. In this work, a variety of 
locations of submerged source and receiver, with separations of less than $50 \mathrm{~km}$, is considered so that different types of ray propagation must be examined. Given a particular profile, procedures are prescribed for constructing a simpler profile for which all significant ray geometric and acoustic quantities, such as ray types, numbers, phase, and amplitudes, are either identical or virtually so. Also, the sensitivity of performance measures for horizontal linear receiving arrays to profile selection is studied. Conditions on array length and source-receiver range and orientation are developed for which a simpler profile may replace a given profile, and still maintain essentially equivalent array performance. [Work supported by ONR.] ${ }^{\text {st }}$ Present address: Siena Colledge, Loundonville, NY 12211 .

\section{9:11}

II10. Analysis of short-range sound transmission through fronts in shallow water. T. H. Rousseau, M. J. Jacobson, and W. L. Siegmann (Rensselaer Polytechnic Institute, Troy, NY 12181)

Effects of sound-speed variations, produced by fronts in shallowocean regions, on short-range ray transmissions between fixed source and receiver are investigated. A parametric model is used in which the front is represented by a sound-speed jump in an otherwise constant-speed channel. The propagation and frontal models are sufficiently general to permit determination of acoustical effects for fronts of varying strengths and for arbitrary oreintations and positions relative to the propagation range. Frontal influences on travel time and geometrical spreading loss are determined, and expressions for per-ray and total field quantities are developed for various source and receiver depths. Using our results, it is shown how the determination of the location of a source is influenced by the presence of a front. Further, it is demonstrated how predictions of the location, strength, and orientation of fronts may be obtained from acoustic receptions at a receiver using the known location of a source. [Work supported by ONR.]

\section{9:15}

II11. Model experiments on the spatial altering of modes. Yunyu Wang(Department of Geology and Geophysics, University of Wisconsin, Madison, WI 53562 and Institute of Acoustics, Academia Sinica, Peking, People's Republic of China), J1-xun Zhou, Hong-hai Li, Zhen-zhong Zhang, and Shen-li Jiang, Er-chang Shang (Institute of Acoustics, Academia Sinica, Peking, People's Republic of China), Qinghua Bao, Long-jiang $\mathrm{Zu}$, and Guo-guang Yan (Oceanographic Institute of Shandong, People's Republic of China)

The spatial filtering of modes is very important both for theoretical and practical purposes. Spatial filtering of modes can verify the normalmode theory and is also a way to match the waveguide fields. It will have an important application in the investigation of the interaction between the sound wave and environmental factors such as the acoustic characteristics of ocean bottom, internal wave,...etc., because it is easier to study separate modes. In this paper, the model experiments include the following: (1) The spatial filtering of modes in a long water tank without absorbers on the side wall. (2) The suppression of the near interference point source by means of selected mode. (3) The effects of surface waves on each individual mode and the sum of all modes at a point receiver.

\section{9:19}

II12. Normal mode filtering in shallow water. En-cen Lo, Jixun Zhou(Institute of Acoustics, Academia Sinica, Peking, People's Republic of China), and Er-Chang Shang (Marine Physical Laboratory, Scripps Institution of Oceanography, La Jolla, CA 92093)

The filtering of mode $I$ and mode 2 by using a vertical array of nine hydrophones has been realized in the frequency range of $250-800 \mathrm{~Hz}$ in the shallow water of the Yellow Sea with a near-perfect isovelocity condition. The eigenfunctions of the mode were calculated by using two parameters $(P, Q)$ to describe the characteristics of the bottom reflections approximately at small grazing angles. The results of the mode filtering were quite good: (1) The group delay measurements of mode 1 and mode 2 agree well with the theoretical value calculated by using the bottom reflection phase shift parameter $P,(2)$ The parameter $Q$ of the bottom reflection loss can be extracted from the amplitude ratio of mode 1 and mode 2 . The extracted values of $Q$ were quite near to the values of $Q$ obtained by other approaches.

\section{9:23}

II13. Some theoretical problems on mode filtering in shallow water. ErChang Shang (Marine Physical Laboratory, Scripps Institution of Oceanography, La Jolla, CA 92093) and Tain-Fu Gao (Institute of Acoustics, Academia Sinica, Peking, People's Republic of China)

Since the mode filtering is a certain sort of "space filtering," the matter of the utmost concern is the "side lobe" produced by some simplified processing in practice instead of the ideal orthogonal operation. In this paper the following problems were discussed theoretically: (1) "side lobe" produced by using an integration in water column instead of half-space; (2) "side lobe" produced by using a finite discrete sampling; (3) "side lobe" produced by using a hard-clipped eigenfunction; (4) "side lobe" produced by the inclination of the vertical array. Some analytic results and numerical examples were shown for a special case in which the sound-speed profile is isovelocity in water column. It was found that the effect of array inclination was serious and a phase compensation approach was proposed.

\section{9:27}

II14. A coupled mode solution for acoustic propagation in a waveguide with stepwise depth variations. Richard B. Evans (Ocean Data Systems, Inc., 6000 Executive Boulevard, Rockville, MD 20852 and Naval Ocean Research and Development Activity, NSTL Station, MS 39529)

A coupled mode solution is formulated for the problem of acoustic propagation in a cylindrically symmetric ocean divided, in range, into a finite number of adjoining Pekeris waveguides of differing water depths. The problem is discretized by assigning a pressure release boundary condition at some large finite depth and including sufficient attenuation to exclude any significant energy returning to the water. This formulation includes backscatter from the depth variations of the waveguide and full cross coupling between a finite number of propagating and nonpropagating modes determined by the range and strength of the depth variations. Numerical results based on an implementation of this solution are presented. [Work supported by NORDA.]

\section{9:31}

II15. An improved numerical ordinary-differential-equation solution to the parabolic wave equation. Ding Lee (Naval Underwater Systems Center, New London, CT 06320) and Kenneth Jackson (University of Toronto, Toronto, M5S 1A7 Canada)

The solution of the parabolic wave equation can be approximated by the solution of a stiff Initial Value Problem (IVP) associated with a system of Ordinary Differential Equations. The Generalized Adams Methods (GAM) based upon a stable rational approximation to the matrix exponential has been shown to be an effective method for solving this problem. However, previous implementations of the GAM suffered from severe storage limitations because of the use of an explicit rational approximation to the matrix exponential. In this presentation, we describe a new implementation of the GAM that is based upon an implicit use of a "Restricted-Pade" approximation to the matrix exponential. This implementation is particularly effective for problems for which the associated matrix is banded, as is the case for the IVP approximating the parabolic wave equation. A problem of propagation in a wedge shaped region is presented to illustrate the strong limitation of the previous implementation and how this limitation is removed in the new implementation. [Work is supported by ONR.]

II16. A comparison of two range-dependent parabolic equation models: IFD and split-step. Ding Lee (Naval Underwater Systems Center, New London, CT 06320), Martin H. Schultz (Department of Computer Science, Yale University, New Haven, CT 06520), and Kenneth E. Gilbert (Naval Ocean Research \& Development Activity, NSTL Station, MS 39529)

The introduction of the IFD (implicit finite-difference) model for the prediction of propagation loss offers users an additional tool for solving the parabolic wave equation. Numerical results comparing the IFD and split-step models for a set of selected problems using the VAX 11/780 computer are presented. In particular, we compare these two models with 
respect to generality, accuracy, speed, user effort, flexibility, reliability, and other features. [Work supported jointly by NUSC and NORDA.]

\section{9:39}

II17. Attenuation of low- and high-frequency waves in a random medium. Alan R. Wenzel (Naval Ocean Research and Development Activity, Code 340, NSTL Station, MS 39529)

A theoretical analysis of the wavefield radiated by a point source in a one-dimensional random medium has been carried out. Approximate expressions for the mean intensity and mean energy flux as a function of propagation range, based on general results obtained previously, have been derived for the limiting cases of low and high frequencies. These expressions show that, in both cases, the mean intensity and mean energy flux decrease more rapidly with range than would be the case in the absence or randomness. This more rapid rate of decrease with range can be interpreted as an excess attenuation. These results are similar, in general, to those obtained previously for the intermediate-frequency case. The low-frequency results are found to be in qualitative agreement with observations of excess attenuation of sound propagating through turbulence. At high frequencies, the results indicate that the excess attenuation is always much smaller than the attenuation due to absorption. This is also in agreement with observations, which generally show no significant excess attenuation at high frequencies. [Work supported by NORDA.]

\section{$9: 43$}

II18. Signal statistics near a caustic in the presence of fluctuations. Thomas L. Clarke (Ocean Acoustics Laboratory, AOML, 4301 Rickenbacker Causeway, Miami, FL 33149)

Signal fluctuations in the field near a caustic produced by random inhomogeneities in sound speed are examined. Statistics are calculated by Monte-Carlo simulation based on a phase-screen approximation. The diffraction parameter for the phase-screen approximation serves to generalize the $\boldsymbol{\Lambda}$ parameter. The statistics are found to depend on the strength of the fluctuations in a complicated way when the generalized difiraction parameter is near unity. In particular, the scintillation index is large at intensity minima even for weak fluctuations.
I119. Short-range, high-frequency acoustic propagation fluctuations. Robert J. Vent and G. Thomas Kaye (Naval Ocean Systems Center, San Diego, CA 92152)

A series of one-way high frequency acoustic propagation experiments were conducted off southern California in an area east of San Clemente Island. Source and receiver were located at depths of 137 and $107 \mathrm{~m}$, respectively, below the diurnal thermocline. The source frequency was 30 $\mathrm{kHz}$; the pulse duration was $0.5 \mathrm{~ms}$ and the pulse repetition rate was 0.5 $\mathrm{s}^{-1}$. A series of fixed ranges were used extending from 180 to $920 \mathrm{~m}$. In addition, temperature fluctuations were observed over the experiment depth range with a thermistor array. For a representative data set, energy density spectra of the acoustic and temperature fluctuations reveal similar forms. At a range of $385 \mathrm{~m}$ with a record length of $1 \mathrm{~h}$ (1800 transmissions), the standard deviation of the received acoustic intensity was $0.5 \mathrm{~dB}$ with a coefficient of variation of $12 \%$. The sound-speed fluctuation patch size was calculated to be $1 \mathrm{~m}$. [Work supported by NAVSEA.]

9:51

II20. Propagation in a random medium: An experiment using heating and cooling in a large water tank. Joe $W$. Posey and Coleman Levenson (Naval Ocean R\&D Activity, NSTL Station, MS 39529)

One millisecond tone bursts in the frequency range 0.8 to $2.0 \mathrm{MHz}$ were propagated to a range of $10 \mathrm{~m}$ through a water tank having a $3.7 \times 3.7 \mathrm{~m}$ cross section. Sound speed distribution along the propagation path was randomized by either heating or cooling. The heating technique has been used by several previous investigators. This method has been criticized because degassification bubbles rise through the propagation path and may act as resonant scatterers. The cooling approach does not produce bubbles. A three-dimensional, fast response thermistor array was used to measure temporal and spatial statistics of the temperature fluctuations. Variations in the complex amplitude of received acoustic signals are compared with Wenzel's theory [AIAA paper 75-546 (1975)]. He defines the coherent wave as having the mean complex amplitude of the total wave and derives a conservation relation for the sum of coherent and incoherent wave energy. He predicts that coherent energy will decay exponentially with characteristic length and mean-square amplitude of sound speed fluctuation as well as with range and signal frequency squared.

\title{
THURSDAY MORNING, 11 NOVEMBER 1982
}

CAPE CANAVERAL AND VOLUSIA ROOMS, 9:00 TO 11:35 A.M.

\section{Session JJ. Engineering Acoustics III: New Sensors}

\author{
T. L. Poteat, Chairman \\ Bell Laboratories, 600 Mountain Avenue, Murray Hill, New Jersey 07974
}

Chairman's Introduction-9:00

\section{Invited Papers}

9.05

\begin{abstract}
JJ1. Formation of silicon microstructures for high performance integrated sensors. $K$. Wise (Department of Electrical and Computer Engineering, University of Michigan, Ann Arbor, MI 48109)

Recent progress in the development of sophisticated, low-cost microcomputers has stimulated the adoption of electronic instrumentation and control in many areas not formerly involved with electronics. In most of these new applications, the realization of the desired system has been found to be limited not by microcomputer performance, but by the lack of suitable sensors to couple the computer to a nonelectronic world. The most promising solution is to extend integrated circuit technology to allow the formation of the needed transducers together with their interface circuitry on a single monolithic chip, constituting an integrated sensor. The ability to precisely shape silicon in three dimensions in an essential requirement in the realization of a variety of
\end{abstract}


transducers. This paper will review and illustrate the available techniques for chemically or electrochemically shaping silicon. The development of a chronically implantable array of recording electrodes for the study of signal processing in the brain and, perhaps, the eventual control of neural prostheses will be discussed. Fabrication techniques and trade-offs involved in the realization of high-performance integrated sensors will also be given.

\section{9:35}

JJ2. Integrated circuit pressure transducers. Wen H. Ko, C. D. Fung, B.-X. Shao, and W. J. Shen (Electronic Design Center, Case Western Reserve University, Cleveland, OH 44106)

Solid state electronic and integrated circuit technology can be used to design and fabricate miniature, high performance transducers. Two examples of silicon diaphragm pressure transducers, with electronic components on the chip, will be discussed. (1) A capacitive pressure transducer for the measurement of very smal deformation of a silicon diaphragm has been studied. This transducer has the potential for better long-term stability, higher sensitivity, and less mechanical interference. These theoretical expectations have been confirmed by a pilot design. Two successful designs incorporating the oscillator, capacitor bridge, and amplifier circuits on the chip are now being evaluated, and the results will be discussed. (2) Piezoresistive pressure transducer design considerations, fabrication processes, and evaluated results will be presented. This mm-size device can be used for both differential and absolute pressure measurements. By integrating electronic circuits on the transducer chip, a new approach to transducer design has been developed that can upgrade the performance of the transducer and generate a new family of "intelligent transducers." Some of the functions and advantages of this approach will be briefly discussed.

\section{0:05}

JJ3. Microelectrode arrays for neurostimulation fabricated by integrated circuit technology. $R$. L. White, L. A. Roberts, and O-H. Kwon (Department of Electrical Engineering, Stanford University, Stanford, CA 94305)

We are engaged at Stanford University in the development of an auditory prosthesis which works by direct electrical stimulation of the auditory nerve. This project must confront many questions on the nature of speech processing, speech comprehension, and the representation of speech as electrical stimulation patterns on the auditory nerve. It is also necessary to develop a microelectrode array for delivery of the appropriate electrical stimulation pattern onto the auditory nerve. While it is possible both in principle and practice to fabricate such arrays using fine wire bundles, it is clearly attractive to utilize the high-resolution high-precision photolithographic technology common to integrated circuit fabrication to achieve arrays which are electrically and mechanically optimal and are reproducible and stable. For the cochlear prosthesis we have developed rigid electrode arrays using a sapphire substrate, tantalum conductors, platinum electrodes and tantalum pentoxide and silicon nitride insulation. We have also developed a flexible electrode array using a liquid polymer and platinum conductors. Comments will be made on problems solved in developing these fabrication techniques and on their extensibility to electrodes for other biostimulation applications.

\section{Contributed Papers}

\section{0:35}

JJ4. Acoustic response of a twisted nematic liquid crystal cell. W. Hamidzada and S. V. Letcher (Department of Physics, University of Rhode Island, Kingston, RI 02881)

A thin layer of liquid crystal can be used as a simple two-dimensional detector of ultrasound. The acoustic beam disturbs the molecular alignment of the liquid crystal, which allows changes in light transmission when the cell is placed between crossed polarizers. Most studies have concentrated on nematic liquid crystals with optic axis along the layer normal. We have studied the acoustic response of a new type of cell developed for electro-optic displays [D. Berreman and W. Heffner, J. Appl. Phys. 52, 3032-3039 (1981)], which is a twisted nematic with oblique attachment angle at the layer surfaces. We find that this cell is generally more sensitive than the homeotropic nematic and has a faster response time. The cell has the additional feature of having two stable molecular configurations that satisfy the same boundary conditions. Under certain conditions the acoustic beam can drive the system from one state to the other, providing a memory feature for the detector.

\section{0:50}

JJ5. Piezoelectric polymer microphones and receivers of improved efficiency. Preston Murphy, Guy Maurer (Lectret SA, 1217 MeyrinGeneva, Switzerland), and Geoffrey Davies (Department of Physics, University of Leeds, Leeds, England)

Piezoelectric plastic film transducers have been designed to operate as microphones and receivers in new communications equipment such as electronic telephones. A theory was developed for a telephone type receiv- er using a thin, circular membrane, curved by air pressure and driving a coupling volume with significant reaction effects. A figure of merit was derived where the acoustic output level per input voltage was proportional to $\bar{d}\{Y /[t(1-v)]\}^{1 / 2}$, where $\bar{d}$ is the mean piezoelectric coefficient $\left(d_{33}\right.$, $d_{32}, t$ is the diaphragm thickness, $Y$ is Young's modulus, and $v$ is Poisson's ratio. Experimental results were in general agreement with theory. A new transducer design of improved efficiency was developed with two circular diaphragms bonded in the center, spaced at the periphery, and connected to operate in a push-pull mode. Either series or parallel connection could be used to match output level and impedance to following circuitry. The efficiency of receivers with correct telephone bandwidth was about 100 $\mathrm{dB} / \mathrm{V}$. This is about $10 \mathrm{~dB}$ higher than receivers with auto-supported or foam-supported diaphragms. Microphone sensitivities were typically $-52 \mathrm{~dB} / \mathrm{Pa}$. The design was shown to be thermally stable and resistant to severe environmental conditions.

\section{1:05}

JJ6. A foil electret transducer for use under varying static pressure. I. J. Busch-Vishniac, J. E. West, and R. A. Kubli (Acoustics Research Department, Bell Laboratories, Murray Hill, NJ 07974)

We have built a foil electret microphone for use under-the-cuff in an automatic blood pressure monitoring system. The microphone uses a 50$\mu$-thick FEP diaphragm charged to $-250 \mathrm{~V}$ and suspended $35 \mu$ above a back electrode. A flat sensitivity over a pressure range of 40 to $250 \mathrm{~mm} \mathrm{Hg}$ $\left(5.3 \times 10^{4}\right.$ to $\left.3.3 \times 10^{5} \mathrm{dyn} / \mathrm{cm}^{2}\right)$ is required for this transducer. The sensitivity is maintained at a constant level by allowing the first contact between the electret and the back electrode to occur at $20 \mathrm{~mm} \mathrm{Hg}$. Although the microphone senistivity changes quickly from 0 to $20 \mathrm{~mm} \mathrm{Hg}$, it 
is relatively flat in the pressure range of interest. This design technique canbe used to provide a flat sensitivity for any foil electret microphone subjected to a varying static pressure.

11:20

JJ7. Feasibility of optictet hydrophones using multimode optical fibers as sensing elements. Peter Shajenko (Naval Underwater Systems Center,
New London, CT 06320)

The use of multimode optical fibers in an interferometer to sense the strain imposed on the fiber was experimentally demonstrated. A phase shift of $2 \times 10^{-4} \mathrm{rad}$ with a $1.4-\mathrm{m}$-long optical fiber was measured. Use of low-coherent light sources such as an LED to reduce fiber noise, improve performance, and simplify design of an optical hydrophone is discussed.

THURSDAY MORNING, 11 NOVEMBER 1982:

DADE AND FLORIDA KEYS ROOMS, 9:00 A.M. TO 12:05 P.M.

\title{
Session KK. Physical Acoustics V and Musical Acoustics III: Measurement of Sound Fields from Complex Sources
}

Wayne M. Wright, Co-Chairman

Physics Department, Kalamazoo College, Kalamazoo, Michigan 49007

\author{
Arthur H. Benade, Co-Chairman \\ $-1$. \\ $\Xi$ \\ $-\dot{1}-$ \\ Physics Department, Case Western Reserve University, Cleveland, Ohio 44106 \\ Chairman's Introduction-9:00
}

$:$

Invited Papers

:

9:05

KK1. Sound radiation measurement with nearfield holography. J. D. Maynard and E. G. Williams (Department of Physics, The Pennsylvania State University, University Park, PA 16802)

With holography, an entire 3D wave field can be reconstructed from data obtained on a single 2D surface. Such high information content has made holography a powerful research tool, and useful extensions from its original optical domain to other wave fields, such as sound, have naturally been pursued. However, it has usually been assumed that the resolution of a holographic reconstruction is limited by the wavelength of the radiation; this limitation severely diminishes the usefulness of holography for sound fields when the wavelengths are many times larger than the objects to be imaged. We have developed a new technique called nearfield holography, which eliminates the wavelength resolution limit and in addition permits a determination of: (1) the complete sound pressure and particle velocity fields produced by the source; (2) the mode of vibration of the surfaces of the source; (3) the vector intensity field (showing flow of acoustic energy) around the source; (4) the farfield directivity pattern; (5) the total power radiated into a half-space. A particularly interesting application of nearfield holography occurs in the study of sound radiation from musical instruments. [Work supported by ONR.]

9:35

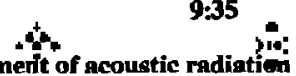

KK2. Computer-mediated measuremenit of acoustic radiation fields. Gabriel Weinreich (Randall Laboratory of Physics, University of Shichigan, Ann Arbor, MI 48109)

By parametrizing an acoustic field as an expansion in solutions of the wave equation, one can not only predict the field at points that have not been measured, but also obtain a powerful connecting link to the detailed physics of the mechanical systems with which the field interacts. To provide such a description of an actual field one must measure its amplitude and phase at sufficiently many points, and sufficiently many frequencies, to compute the necessary coefficients; even if both domains are intelligently truncated, the amount of data to be collected and processed remains enormous. Fortunately, modern electronics and computers make such an approach practical. Illustrations will be given of (a) a system that expands a measured radiation field in spherical waves, separating outgoing from incoming waves, and classifying each by its spherical harmonic angular dependence; (b) a system that measure modiative monopole and dipole moments of a complex vibrating structure, whose frequency dependence zan then be analyzed to yield the radiative contributions of the normal modes. [Work supported by NSF.] 
KK3. Measurement of room-averaged spectra from complex sources. A. H. Benade (Physics Department, Case Western Reserve University, Cleveland, $\mathrm{OH}$ 44106)

When a complicated source is turned on in a room, each of the earliest reflections arriving at the detector carries spectral information about the sound as radiated in a particular direction. However, the random phase shifts and attenuations of these successive arrivals lead ultimately to a steady-state signal whose spectral components each have a Rayleigh distribution with a spread of $\pm 5.2 \mathrm{~dB}$ around a mean value obtainable via many readings taken at suitably spaced points throughout the room. Thus at least 27 properly chosen samples are required to give a precision of $\pm 1 \mathrm{~dB}$. In some cases FFT analysis of the arrivals before about $\left(T_{\text {rev/s }}\right)$ can provide roughly equivalent precision with fewer samples. There are indications that musical perception involves processes related to both the buildup-epoch and steady-state spatial-sampling procedures of the laboratory. Constraints on room size, reverberation time, intersample distance, source-to-detector distance, etc. (following Schroeder, Waterhouse, Lubman...) will be outlined. Examples of buildup-epoch and steady-state spatial-sampling measurements will be presented and compared. [Work supported by NSF.]

\section{0:35}

KK4. Recent progress in measurement methods for studying acoustic radiation. Thomas $H$. Hodgson (Center for Sound and Vibration, North Carolina State University, Raleigh, NC 27650)

The need to locate and describe sources of noise from complex machinery has meant that recently there has been tremendous progress in measurement techniques. This is particularly true in the areas of acoustic intensity measurement, both in the free-field and on the radiating surface, and in coherence function signal processing and modal analysis. These methods have been applied to both steady-state and transient noise sources such as impact machinery. This paper will review this progress in measurement methods for studying acoustic radiation which are very applicable to analysis of the source radiation problem in musical acoustics, where the sound sources can be both complex and transient in nature.

\section{1:05}

KK5. Impedance and impulse response measurements using low-cost components. M. I. Ibisi (University of Jos, Jos, Nigeria) and A. H. Benade (Case Western Reserve University, Cleveland, OH 44106)

A thin 27-mm-o.d. disk transducer (from Radio Shack 273-060 signaler) is RTV sealed to the end of a measurement tube whose $15 \mathrm{~mm}$ i.d. not only approximates the transducer disk's nodal circle but also the i.d. of a clarinet, sax, or trumpet mouthpiece. A 10-mm-o.d. electret microphone element (Radio Shack 270-092) mounted through the tube wall near the piezo driver picks up the resulting pressure signal. When the high mechanical impedance driver is excited by a sinusoidal sweep via a 6-dB/ oct integrator, the resulting pressure signal gives an excellent view of the input impedance of the air column over the musically useful range to 3.5 $\mathrm{kHz}$ since the driver resonance is at $\omega_{0} / 2 \pi \simeq 5 \mathrm{kHz}$ and band width $g \simeq \omega_{0} /$ 6. A ramplike drive voltage (running between op-amp supply limits) of the form $A\left(1-e^{-g^{t} / 2}\right)$ for $\theta \leqslant t \leqslant 2 \pi / \omega_{0}$, and constant thereafter, gives a clean pressure pulse $\simeq$ const. $\left(1-\cos \omega_{0} t\right)$ whose $\mathrm{FWHM} \simeq 0.1 \mathrm{~ms}$ is , convenient for impulse response studies. Drive signal spectrum, piezoelectric coupling, and driver-to-tube coupling each minimize transducer secondmode ringing effects. Driver-to-mike proximity effects are small, visible, calculable, and easily allowed for in both impulse and sinusoidal drive usage. The driver is not quite rigid, perturbing the air column slightly, but corrections are straightforward. Applications for research and in teaching labs will be described. [Assisted by NSF.]

\section{1:20}

KK6. Full field visualization towards developing improved ultrasonic test procedures. C. P. Burger, I. Miskioglu (Department of Engineering Science and Mechanics, Iowa State University, Ames, IA 50011), and R. G. Hughes (Naval Research Laboratory, Washington, DC 20375)

Improved procedures for quantitative ultrasonic evaluation of materials and structures depend upon improved knowledge of the ways in which acoustic waves in solids interact with geometric details and with typical defects. Full field visualization of the dynamic elastic field as it propagates through a structure is a powerful physical aid in developing such an improved understanding. This paper describes how a multilaser, CranzSchardin type, high-speed polariscope was used to photograph the full field dynamic progression of a Rayleigh surface wave past geometric details at the surface of a solid. The resultant spatial information of the surface stresses is used to extract frequency information for the incident, reflected and transmitted waves at a step on the surface. The characteristic features of the FFT that relate to the step height is identified and confirmed in ultrasonic tests on steel samples. This integrated, three pronged, experimental/numerical approach holds great promise towards developing more effective ultrasonic test procedures. [Work supported by NA VSEA, NOOO14-81-CO2475.]

\section{1:35}

KK7. Detection of broadband acoustic pulses in liquids using wideband differential interferometry. Avinash $O$. Garg, Richard $O$. Claus, and George D. Dockery (Department of Electrical Engineering, Virginia Polytechnic Institute and State University, Blacksburg, VA 24061)

Several authors have reported the detection of continuous ultrasonic waves in transparent media by interferometric transmission techniques [C. H. Palmer, R. O. Claus, and S. E. Fick, Appl. Opt. 16, 1849 (1977); D P. Jablonowski, Appl. Opt. 17, 2064 (1978)]. In this paper, the measurement of broadband acoustic pulses in a water tank by a wideband differential optical system is discussed. The two coherent light beams in this system are narrowly collimated and propagated through the tank along lines parallel to the generated ultrasonic wavefronts and normal to their direction of propagation. A change in the index of refraction of the water caused by the acoustic field at the location of one optical beam alters the optical phase difference between the beams and is interferometrically detected. Since the optical beams are separated, the acoustic pulse modulates them at different times and two detected differential signals of opposite sign are observed. Beam separation determines the lower frequency response limit of the system; upper frequency response is limited to approximately $100 \mathrm{MHz}$ by the diameter of the optical beams. Applications in the scanning of three-dimensional acoustic fields is indicated. [Work supported in part by NASA and NSF.]

\section{1:50}

KK8. Volume perturbers as possible sound field randomizers in roomaverage spectrum measurement procedures. $I$. $M$. Lindevald and $A$. H. Benade (Physics Department, Case Western Reserve University, Cleveland, OH 44106)

The use of moving volumes (people) instead of rotating vanes for sound field randomization has been proposed as a plausible and convenient technique. A perturbing volume $V_{\rho}$ in a room of volume $V_{0}$ shifts the frequen- 
cy of the Imn mode by an amount $(\Delta f / f)_{\text {Imn }}$ $=\int v_{r}(U-K)_{l m n} d V / \int v_{n}(U+K)_{l m n} d V$, where $U$ and $K$ are the modal potential and kinetic energy densities of the sound field. Computer experiments confirm that when $V_{\rho}$ is moved throughout a room, the rms frequency shift is given by $(\Delta f / f)^{\mathrm{rms}}=3\left(V_{p} / V_{0}\right) H(k a)$. For a cube of side $a$, $H \simeq 1$ for $k a<1$, falling basically as $(1 / k a)$ above this. For $V_{p}$ a thin square of side $a$, or bar of length $a$, the same asymptotic relations hold, but the transition to $(1 / k a)$ behavior is much less abrupt. If $(\Delta f / f)^{\mathrm{rms}}>$ the mean intermode frequency spacing $\left(c^{3} / 4 \pi V_{0} f^{3}\right)$, one expects adequate randomi- zation of the room modes for frequencies above $f_{p}=c /\left(12 \pi V_{\rho}\right)^{1 / 3}$ provided $V_{p}$ is displaced sufficiently between recordings of the spectral samples. Measurements of spectra using fixed source and microphone but moving $V_{p}$ as outlined show however that the observed mean spectrum has a variance only slightly less than the $\pm 5.2 \mathrm{~dB}$ value associated with a single reading. That is, the expected randomization due to moving $V_{\rho}$ hardly takes place. Other types of experiment confirm this. Possible reasons will be discussed. [Assisted by NSF.]

THURSDAY MORNING, 11 NOVEMBER 1982

ORANGE ROOM, 9:00 A.M. TO 12:05 P.M.

\title{
Session LL. Speech Communication V: Speech Production
}

\author{
Thomas Shipp, Chairman \\ Veterans Administration Hospital, 42nd Avenue \& Clement Street, San Francisco, California 94121
}

Chairman's Introduction-9:00

Contributed Papers

9:05

LL1. A phonetically relevant auditory-perceptual space. James D. Miller (Central Institute for the Deaf, 818 S. Euclid, St. Louis, MO 63110)

The vowels, based on averages of Peterson and Barney [J. Acoust. Soc. Am. 24, 175-184 (1952)], can be described independently of the age and sex of the talkers in a space with axes: $x=\log (F 3 / F 2), y=\log \left(F 1 / F 0^{\prime}\right)$, and $z=\log (F 2 / F 1)$, where $F 0^{\prime}=a F 0 . F 1, F 2$, and $F 3$ are the center frequencies of the first three formants and $F 0$ is the fundamental. The constant $a$ varies across speakers, but the average value for men is 1.478 times that for children and women. So described, the vowels fall within a tetrahedron, whose properties will be described, and their spatial organization appears to reflect their perceptual and articulatory organization and to suggest rules for the enlargement or compression of their spectra. Distances between vowels are in $\log$ frequency and are easily converted to cents, semitones, or octaves. As examples, the neighboring vowels, $/ \mathrm{I} /$ and $/ \varepsilon /$, are separated by 0.76 octaves while the distant vowels, $/ \mathrm{a} /$ and $/ i /$ are separated by 3.2 octaves. New vowel data and possibilities for locating the consonants in the space will be mentioned. [Supported by NINCDS Grant NS 03856.]

\section{9:20}

LL2. Planning speech for execution at different tempos. William E. Cooper, Audrey Ham, Kristina Damon, and Carlos Soares (Department of Psychology and Social Relations, Harvard University, Cambridge, MA 02138)

A study was conducted to examine the influence of speaking rate on the occurrence of palatalization across word boundaries, fall-rise patterns of fundamental frequency, and pausing. Eighteen speakers, nine of whom were characterized as normally fast speakers, uttered preselected sentences at normal, fast, and slow rates. The frequency of palatalization was greater for characteristically fast speakers and for fast rates of speech within speakers. Palatalization was reduced in the immediate vicinity of emphatic stress, most markedly when such stress appeared on the $/ y /$ word, replicating earlier results [W. E. Cooper, C. Egido, and J. M. Paccia, J. Exp. Psych.: Hum. Perc. Perf. 4, 264-272 (1978)]. Analyses of fundamental frequency and pausing for these utterances and for a related corpus of naturally elicited speech will be discussed in terms of implications for a model of speech planning and execution. [Work supported by NIH.]
9:35

LL3. Experimental manipulation of speaking rate for studying temporal variability in children's speech. Bruce $L$. Smith, Steven $H$. Long, and Michael D. Sugarman (Speech and Language Pathology, Northwestern University, 2299 Sheridan Road, Evanston, IL 60201)

Children's speech typically exhibits both longer segment durations and greater inter- and intra-subject variability than the speech of adults. One hypothesis which has been proposed to account for the greater variability is that children have less-refined neuromotor control capabilities and do not move their articulators with the same accuracy that adults do. Another hypothesis is that children's increased variability is essentially a statistical artifact which occurs because of the greater range of durations likely to be associated with longer segment durations. In the past, statistical methods alone (e.g., "relative variance" and "coefficient of variation") have been used in an attempt to compensate for between-group duration differences when comparing variability in the speech of adults and children. In the present study, durational factors were also experimentally manipulated by having three groups of children (ages 5, 7, and 9) and a group of adults produce ten repetitions of a sentence at each of three different speaking rates: normal, fast and slow. Within- and betweengroup comparisons of variability under these conditions were investigated. Results will be discussed in relation to the above two hypotheses.

9:50

LL4. The influence of consonant environment on $F 0$ from voicing onset to vowel target in the speech of children. Ralph $\mathrm{N}$. Ohde (Bill Wilkerson Hearing and Speech Center and Vanderbilt University \$chool of Medicine, 1114 19th Avenue South, Nashville, TN 37212)

Although research on adult speech has manifested systematic effects of consonant environment on the fundamental frequency $(F 0)$ of vowels, little is known about the nature of these influences in children. In order to determine the effects of consonant environment on $F 0$ in children, three girls and three boys between 8 and 9 years recorded five repetitions each of voiceless aspirated $/ \mathrm{p}^{\mathrm{h}}, \mathbf{t}^{\mathrm{h}}, \mathrm{k}^{\mathrm{h}} /$ voiceless unaspirated $/ \mathrm{sp}, \mathrm{st}, \mathbf{s k} /$, and voiced $/ b, d, g /$ stops in combination with the vowels $/ i, e, u, o, a /$. Fundamental frequency was measured for the first five glottal periods and vowel target, and voice onset time (VOT) was determined for each utterance. The findings for VOT revealed nearly identical results for voiceless unaspirated stops and voiced stops, whereas differences between voiceless unaspirated 
and voiceless aspirated stops for labial, alveolar, and velar features were 61,65 , and $59 \mathrm{~ms}$, respectively. Despite the similarity in VOT between voiceless unaspirated and voiced stops, $F 0$ contours of voiceless unaspirated stops were more similar to voiceless aspirated than voiced stops. In general, there was a decrease in $F 0$ from the first glottal period to the second glottal period for all voicing conditions. Moreover, $F O$ was usually higher in the environment of voiceless stops than voiced stops, although this was not unanimous across subjects. The findings will be discussed relative to developmental and coarticulatory processes, and sex differences. [Work supported in part by NINCDS.]

\section{0:05}

LL5. The effects of linguistic context on temporal and $F 0$ properties of speech. Ralph N. Ohde (Bill Wilkerson Hearing and Speech Center and Vanderbilt University School of Medicine, 1114 19th Avenue South, Nashville, TN 37212)

Research examining acoustic properties of speech frequently use isolated syllables or syllables embedded in carrier phrases for analysis. Although it has been argued that these contexts represent rather limited speech situations [N. Umeda, J. Acoust. Soc. Am. 70, 350-355 (1981)], it is not clear that the influences of these contexts on speech are comparable. In order to determine the effect of these contexts on several acoustic properties of speech, three male subjects recorded repetitions of voiceless aspirated $/ \mathrm{p}^{\mathrm{h}}, \mathrm{t}^{\mathrm{h}}, \mathrm{k}^{\mathrm{h}} /$ and voiced $/ \mathrm{b}, \mathrm{d}, \mathrm{g} /$ stops in combination with the vowels $/ \mathrm{i}, \mathrm{e}, \mathrm{u}, \mathrm{o}, \mathrm{a} /$ in both isolated CVC syllables and CVC syllables embedded in carrier phrases. Fundamental frequency $(F 0)$ was measured for the first five glottal periods and voice onset time (VOT) was determined for each utterance. The three acoustic properties selected for purposes of comparison were VOT, the percent decrease in $F 0$ from the first to second glottal period, and the absolute value of $F 0$ for the first five glottal periods. The results revealed that the greatest differences in VOT between isolated syllables and syllables produced in carrier phrases were for voiceless stops, i.e., $37 \mathrm{~ms}$ or greater across place of articulation, whereas for the $F 0$ properties variation between these contexts was greatest for voiced stops. In general, however, the findings for $F 0$ were similar in direction to results obtained in fluent reading. [Work supported in part by NINCDS.]

\section{$10: 20$}

LL6. Context-induced vowel duration change and intrinsic vowel duration. Michelle Caisse (Phonology Laboratory, University of California, Berkeley, CA 94720)

This study investigated the pattern of duration change of vowels of different quality (and intrinsic duration) when spoken in contexts which differently affect duration. Stressed vowel duration was measured in five contexts; test stimuli included all 15 English vowels and diphthongs. Duration change induced by context appeared to be independent of intrinsic vowel duration and vowel quality, and was best described as a constant which combines additively with some measure of intrinsic vowel duration. Results of other studies which examined similar data are discussed.

\section{$10: 35$}

LL7. Evaluation of vocal efficiency in voice disorders by calculation of intrapulmonic pressure. Shinzo Tanaka and Wilbur J. Gould (Vocal Dynamics Laboratory, Lenox Hill Hospital, $100 \mathrm{E} 77$ th Street, New York, NY 10021l

Vocal efficiency was investigated by simultaneously measuring the intrapulmonic pressure, the mean flow rate and the sound intensity. The intrapulmonic pressure during sustained phonation has been successfully and noninvasively calculated with a pneumotachograph and a body plethysmograph. Normal and disordered subjects were examined, and the variation of vocal efficiency for each patient from the averaged values of ten normal subjects was investigated. The vocal efficiency is represented by a ratio of the sound power to the aerodynamic power, and the aerodynamic power is expressed in terms of intrapulmonic pressure times mean flow rate. The result suggests that the variation of vocal efficiency can represent overall severity of the voice disorder. The relative contributions of intrapulmonic pressure and mean flow rate to the efficiency can classify the pathological lesions into three types such as leakage of air through the glottis, a mass on the vocal fold, and the stiffness of the vocal fold

\section{0:50}

LL8. Laryngeal function: Electroglottograph, ultra-high-speed films, and speech inverse filtering. A. K. Krishnamurthy, J. M. Naik, and D. G. Childers (Department of Electrical Engineering, University of Florida, Gainesville, FL 32611)

Numerous methods have been devised to assess laryngeal function, including photography, inverse filtering of speech, electroglottography, and others. We have been conducting a series of experiments in which we record simultaneously the electroglottograph (EGG) waveform and the acoustic phonatory signal, while filming vibratory patterns of the vocal folds for both normal subjects and patients with various pathologies and vocal disorders. These data are synchronized via a special timing signal, which is recorded on the audio tape and ultra-high-speed laryngeal films. We present graphical plots of this synchronized data including (1) glottal area measured from the films, (2) the EGG waveform, (3) the speech waveform, and (4) the inverse filtered waveform. These results are being used in various ways including the verification that the EGG signal is indicative of the amount of contact between the vocal folds. Other uses of our results are obtaining better models of the source/tract coupling and the synthesis of more natural sounding speech.

\section{1:05}

LL9. Relationships between vowel air expenditure and fricative air flow in VCV syllables by normally hearing and hearing-impaired speakers. Robert L. Whitehead (National Technical Institute for the Deaf, P. O. Box 9887, Rochester, NY 14623)

In a previous investigation, Whitehead et al. [J. Acoust. Soc. Am. Suppl. 1 64, S51 (1978)] speculated that significantly reduced air-flow rates for intervocalic fricatives produced by some hearing-impaired speakers may have been the result of air wastage on the initial vowel. In the present investigation, the volumes of air expended during production of the initial vowel in VCV syllables by ten normally hearing females, ten moderately to severely hearing-impaired females with intelligible speech and ten profoundly hearing-impaired females with semi-intelligible speech were calculated and compared with the average volume velocities for the intervocalic fricatives. The results indicated that the semi-intelligible hearing-impaired speakers produced the initial vowels with significantly greater air volumes than the other two groups of speakers. Further, for the semi-intelligible speakers, significant negative correlations indicated the existence of a relationship between the volume of air expended on the initial vowel and the average volume velocity for the intervocalic fricative. Such a relationship did not appear to exist for the other groups of speakers. [Work supported by U. S. Department of Education.]

\section{$11: 20$}

LL10. Aerodynamic/timing factors related to the intelligibility of deaf speakers. W. S. Brown, Jr., Donald Goldberg, and Howard Rothman (IASCP and Department of Speech, ASB 63, University of Florida, Gainesville, FL 32611)

The literature describing the speech of the deaf indicates that the inability to learn timing characteristics of consonant and vowel productions is a major cause of reduced intelligibility in oral communication. This study measured several timing gestures for consonant and vowel combinations which have been shown to be essential for intelligibility. The subjects included three groups of speakers between the ages of 12 to 14 years. Group I, which served as the control, consisted of 15 normal hearing school children with normal speech. Group II consisted of 15 prelingual deaf children who were trained predominately in manual communication. Group III consisted of 15 prelingual deaf children who were trained predominately in oral communication. The three groups were compared for differences and similarities in (1) consonant duration, (2) vowel duration, and (3) VOT associated with productions of $\mathrm{CV}, \mathrm{VCV}$, and VC syllables 
spoken in a carrier phrase. The results will be discussed in terms of the deviations of the deaf speech from the normal speech and their relationship to intelligibility.

\section{1:35}

LL11. A comparative study of common chimpanzee and human infant sounds. Harold R. Bauer (Boys Town Institute, $555 \mathrm{~N}$. 30th Street, Omaha, NE 68131)

Chimpanzees observed in Tanzania produced a graded, dynamic sound system ranging from short distance, softer, lower $F 0$ sounds to long distance, louder, higher $F 0$ sounds. A softer and a louder sound category, the whimper and the scream, were selected for further study of $F \mathbf{0}$ and harmonics. From these acoustic analyses and behavioral observations, evidence for a variety of phonatory and resonance patterns were evident in their vocalic sound system which could carry information but lack the segmental contrastivity of human speech. Human infants about a year old were audio and video recorded in the laboratory or home. Vocalic sound durations, $F 0$ contours, and range were much more variable than those observed for chimpanzees. The $F 0$ of human infant sounds often ranged from $300-600 \mathrm{~Hz}$, although occasionally $F 0$ values many times higher were produced making harmonic-formant interactions a critical issue in the comparison with chimpanzee sounds. Fricative, stop, and other consonants not evident in chimpanzee sounds, were common in human infants at 13 months. Selection and reduplication of early word productions suggests the importance of self stimulation, caregiver responsiveness, imi- tation, vocal tract development, and the acquisition of species typical speech motor skills as some important prerequisites to the development of human words. [Support was provided by NIH, NSF and the A. von Humboldt Foundation.]

\section{1:50}

LL12. The roice of dominance. John J. Ohala (Phonology Laboratory, Department of Linguistics, University of California, Berkeley, CA 94720)

Among animals dominance and aggression is conveyed in part by vocalizations with low fundamental frequency $\left(F_{0}\right)$, whereas submission is signalled by cries with high $F_{0}$, e.g., growls and whines, respectively. It has been suggested that low and high resonances of vocalizations should serve the same function and that this helps to explain the origin of the smile, a nonaggressive signal, since retracting the corners of the mouth during a vocalization would raise the resonant frequencies [Ohala, "The acoustic origin of the smile," J. Acoust. Soc. Am. Suppl. 168, S33 (1980)]. Do these acoustic parameters function similarly in human vocalizations? To investigate this, American English listeners were asked to listen to and to determine subjectively the relative "dominance" evident in voice samples that were actually synthetic speech samples devoid of recognizable lexical elements but varying in $F_{0}$ and formant structure. Preliminary results suggest that perceived dominance is correlated inversely with overall $F_{0}$ of voice. Further results will be presented and discussed in relation to current ethological theory. [Supported by the Harry Frank Guggenheim Foundation.]

\title{
Meeting of the Standards Committee S12 on Noise
}

to be held jointly with the

\section{Technical Advisory Group for ISO/TC 43/SC1 Noise}

\author{
K. M. Eldred, Chairman S12
}

\section{P. O. Box 1037, Concord, Massachusetts 01742}

\author{
H. E. von Gierke, Chairman, Technical Advisory Group for ISO/TC 43/SC1
}

\section{Chief, Bionics \& Biodynamics Division, U. S. Air Force AMRL/BB, Wright-Patterson AFB, Dayton, Ohio 45433}

\begin{abstract}
At its first formal meeting, Chicago, Illinois (April 1982), the current status of standards under preparation was discussed. Discussion took place on new standards which might be needed over the next few years. In addition, a report was given on the March 1982 meeting of ISO/TC43/SC1 Noise, for which $\mathrm{S} 12$ is the U. S. counterpart.
\end{abstract}

At this meeting, working group chairpersons will report on their progress under the newly developed plan for the production of noise standards. The interaction with ISO/TC $43 / \mathrm{SC} 1$ will be discussed. 


\title{
Session MM. Psychological Acoustics IV: Tuning Curves and Forward Masking
}

\author{
Walt Jesteadt, Chairman \\ Boys Town Institute, 555 N. 30 Street, Omaha, Nebraska 68131
}

Chairman's Introduction-10:45

\section{Contributed Papers}

\section{0:50}

MM1. Confusion effects in forward masking. Donna L. Neff (Boys Town Institute for Communication Disorders in Children, 555 North 30th Street, Omaha, NE 68131)

Moore and Glasberg [J. Acoust. Soc. Am. 71, 941-948 (1982)] recently stressed that some forward-masked thresholds may reflect the listener's difficulty in discriminating between signal and masker, rather than the detectability of the signal energy. As an extreme example, if the masker were a long series of brief tonal pulses, the detectability of one more identical pulse added to the end of the series would be very low if the level of all pulses were identical. Psychometric functions for such stimuli should show a local minimum, with performance approaching chance in the region of greatest signal-masker confusion. These predictions were tested in several experimental conditions, using various masker-signal combinations, psychophysical procedures, and a contralateral cue (broadband noise) designed to resolve confusion about masker offset. Results confirm the predictions of the "confusion" interpretation of certain forwardmasking effects. However, psychophysical procedure and listener training have a stronger influence on performance than the use of the contralateral cue. [Work supported by NSF, NIH, and the University of Nebraska.]

\section{1:05}

MM2. Interaction of simultaneous and for ward masking for maskers and signals differing in frequency. Walt Jesteadt, Daniel $\mathbf{L}$. Weber, and S. Shane Wilke (Boys Town Institute for Communication Disorders in Children, Omaha, NE 68131)

The combination of a $1000-\mathrm{Hz}$ forward masker and a broadband simultaneous masker produces more masking than would be predicted from the sum of their individual effects [Jesteadt and Wilke, J. Acoust. Soc. Am. Suppl. 1 71, S72 (1982)]. We have extended the original study to include forward maskers above and below the signal in frequency. We measured the threshold for a $1000-\mathrm{Hz}$ signal as a function of masker level ( 30 to $80 \mathrm{~dB} \mathrm{SPL}$ ) for forward masker frequencies from 600 to $1200 \mathrm{~Hz}$ (100-Hz steps below the signal, $50-\mathrm{Hz}$ steps above) with the signal and masker presented in quiet and in an 8-dB No background noise. Nonadditivity was greatest when the forward masker and signal were similar in frequency. We also obtained psychophysical tuning curves for a $45-\mathrm{dB}$ SPL, $1000-\mathrm{Hz}$ signal using forward maskers from 600 to $1200 \mathrm{~Hz}$, with the signal and masker presented in quiet and in noise. As reported by Green et al. [J. Acoust. Soc. Am. 69, 1758-1762 (1981)], the forwardmasked tuning curves were sharper in noise, presumably because the nonadditivity of the two maskers was greatest in the region of the tip of the tuning curve. If the background noise produced an increase in masking by restricting off-frequency listening, we would predict greater nonadditivity when the signal and masker differed in frequency as well as broader tuning curves in noise than in quiet. [Work supported by NIH and NSF.]

\section{1:20}

MM3. Tuning curves and pitch matches in unilateral, low-frequency hearing loss: A case study. Mary Florentine (Communications Research Laboratory, 133 FR, Northeastern University, Boston, MA 02115 and
Research Laboratory of Electronics, 36-730, Massachusetts Institute of Technology, Cambridge, MA 02139) and Adrianus J. M. Houtsma (Research Laboratory of Electronics, 36-755, Massachusetts Institute of Technology, Cambridge, MA 02139)

Psychoacoustical tuning curves and interaural-pitch matches were measured in a listener with a moderately severe hearing loss of primarily cochlear origin below $2 \mathrm{kHz}$. The simultaneous-masked tuning curves at 1 $\mathrm{kHz}$ in his impaired ear show that as the level of the probe increased from 4.5 to $13 \mathrm{~dB} \mathrm{SL}$, (1) the frequency location of the tuning-curve tip decreased from approximately 2.85 to $2.20 \mathrm{kHz}$ and (2) the lowest masker level increased from 49 to $83 \mathrm{~dB}$ SPL. The tuning curves for different probe levels appear to reflect tuning properties of different populations of nerve units. The tuning curve in his normal ear was comparable to data from other normal listeners. The interaural-pitch matches were measured from 0.5 to $6 \mathrm{kHz}$ at $10 \mathrm{~dB}$ SL in the impaired ear and approximately 15 to $20 \mathrm{~dB} \mathrm{SL}$ in the normal ear. Results show reasonable identity matches (e.g., a test frequency in the impaired ear was matched relatively close to the same test frequency in the normal ear), although variability was significantly greater for pitch matches below $2 \mathrm{kHz}$. The pure-tone pitch matches of this listener in the impaired frequency region appear more easily explained in terms of temporal theories than place theories of pitch perception. [Work supported by NIH.]

\section{1:35}

MM4. Tuning curves in the presence of a simultaneous noise masker. Edward A. Cudahy and Daniel Geller (Communicative Disorders, Syracuse University, Syracuse, NY 13210)

Psychoacoustic tuning curves for a $20-\mathrm{ms}, 4-\mathrm{kHz}$ signal were measured in quiet and in the presence of a 750-ms simultaneous noise masker. The signal was presented in the temporal center of the masker. The subjects were three normal listeners. The three noise masker conditions were low-pass $1 \mathrm{kHz}$, high-pass $5.5 \mathrm{kHz}$, and broadband. High-pass and broadband noises had little effect on tuning curves, whereas low-pass noise "detuned" the tuning curve by lowering the high-frequency slope. Implications of these findings for the role of suppression in measurements of frequency selectivity will be discussed.

\section{1:50}

MM5. Psychophysical tuning functions, dichotic stimuli, and pulsation thresholds. David R. Soderquist (Department of Psychology, University of North Carolina at Greensboro, Greensboro, NC 27412)

Using a dichotic mode of stimulus presentation, four psychophysical tuning curves were obtained: input filter pattern, input extension pattern, output filter pattern, and output extension pattern [J. Verschuure, Acustica 49, 288-295 (1981)]. Stimulus parameters for all tuning functions were: durations $60 \mathrm{~ms}$, rise/decay $10 \mathrm{~ms}$, interstimulus interval (delta-t) $0.00 \mathrm{~ms}$ (no overlap between pulsator offset and signal onset). Stimuli were presented in sequential pairs (pulsator then signal), alternating between ears; e.g., left ear (pulsator-signal), right ear (pulsator-signal), left ear (pulsator-signal), etc. The alternation between ears was temporally controlled to insure no overlap (delta-t $=0.00 \mathrm{~ms}$ ) between signal offset in one ear and pulsator onset in the other. Pulsation thresholds were obtained by the 
method of adjustment for each of the four tuning patterns. Results indicate that pulsation threshold patterns could be perceived when the stimuli were presented dichotically, i.e., the perception of "continuity" was synthesized by central processes. Tuning patterns, generated with the dichotic stimuli and based on the centrally synthesized pulsation thresholds, appear to be similar to those in the literature. Contrasts of the four tuning patterns will be presented.

\section{2:05}

MM6. Relation between simultaneous-masking psychophysical tuning curves and cubic-difference-tone amplitudes for $F m<F p$. Susan J. Norton ${ }^{\text {a) }}$ and Craig C. Wier (Department of Speech and Hearing Sciences, University of Washington, Seattle, WA 98195)
The detection of cubic difference tones (CDTs) generated by the masker and probe could influence the shapes of simultaneous-masking psychophysical tuning curves (PTCs) if listeners increase the masker level until the CDT, as well as the probe, becomes inaudible. To test this possibility, simultaneous-masking PTCs were measured for a $125-\mathrm{ms}, 2000-\mathrm{Hz}$ probe at 30,45 , and $60 \mathrm{~dB}$ SPL temporally centered in a 250 -ms masker in four normally hearing listeners using a method-of-adjustment procedure. The amplitudes of CDTs produced by the combination of masker and probe from the low-frequency side of the $\operatorname{PTC}(F m<F p)$ were then determined using pulsation-threshold and cancellation procedures. In all cases, a CDT was measurable, i.e., above masked threshold, indicating that CDT threshold did not establish the tuning-curve masker level. Measurements of CDT amplitude as a function of $L 1$ for fixed $L 2 \mathrm{~s}(30,45$, and $60 \mathrm{dBSPL})$ also suggest that for pulsed masker and probe stimuli, listeners did not rely upon the masking of the CDT in the simultaneous-masking PTC task. [Work supported by the Deafness Research Foundation and NIH.] ${ }^{\text {a) }}$ Present address: Boys Town Institute, 555 N. 30th St., Omaha, NE 68131. 


\title{
Session NN. Architectural Acoustics V: Air-Supported Structures
}

\author{
Wendell D. Anderson, Chairman \\ Coffeen Anderson \& Associates Inc., 5805 Outlook, Mission, Kansas 66205 \\ Chairman's Introduction-1:00
}

Invited Papers

$1: 05$

NN1. Fabric air-supported and tensile roofs for large structures-A review of fabric roof technology. David Geiger (Geiger Berger Associates, P.C., 114 Fifth Avenue, New York, NY 10011)

The use of fabric for the roofs of large audience spaces and similar structures is a relatively new technology. It had its beginning with the large air supported structure for the U. S. Pavilion at the 1970 Worlds Fair in Japan. This technology has continued to produce the very large tensile structure at the Jetta Airport in Saudi Arabia, and the large stadium with an air-supported fabric roof that recently opened in Minneapolis and the similar facility that is nearing completion in Vancouver, British Columbia. A review of the major projects that have utilized this technology during the past twelve years is useful in applying the fabric roof concept to future structures.

\section{1:35}

NN2. Acoustical design considerations for two air-supported structures. John A. Curtis, Parker W. Hirtle, and Thomas R. Horrall (Bolt Beranek and Newman Inc., Cambridge, MA 02139)

This paper presents information on the acoustical design of the Hubert $\mathrm{H}$. Humphrey Metrodome in Minneapolis, Minnesota and the Dalhousie University Athletics Complex (Dalplex) in Halifax, Nova Scotia. The Hubert H. Humphrey Metrodome seats up to 63000 people under an air-supported fabric roof. Major concerns that are discussed were reverberation control and the design of a system of loudspeaker clusters that are suspended from the roof membrane and maintain proper orientation when the structure is moved by changes in wind loading. The main feature of Dalplex, which is located in a residential neighborhood, is a $300 \mathrm{ft}$ by $240 \mathrm{ft}$ stainless steel air-supported roof over the field house and running track. This $\frac{1}{16}$-in.-thick stainless steel membrane presented some unique acoustical problems that had to be addressed, including control of reverberation in the fieldhouse, reduction of the noise of rain on the metal roof to acceptable levels, and reduction of crowd noise transmitted through the roof to nearby houses. These and other acoustical considerations are discussed.

NN3. Acoustical properties of glass fiber roof fabrics. Ron Moulder and Jim Merrill (Owens-Corning Fiberglas, Technical Center, Granville, $\mathrm{OH}$ 43023)

Woven glass cloth that has been coated with a hydrocarbon is used extensively as a roof membrane in air or tension-supported structures. Previous presentations have been made on the acoustical environment of some of these structures. This paper will focus on the acoustical properties of the roof fabric as measured in the laboratory. Sound transmission loss values were measured for two coated fabrics with and without inner liners. Tests were conducted in accordance with the ASTM test method E90-75. Sound absorption tests were also conducted on these same roof configurations in accordance with ASTM test method C423-77. The data generated from these tests should be most helpful to the designer or consultant in determining the acoustical impact both inside and outside fabric roof structures.

NN4. Acoustical problems in large structures employing fabric roofs. Robert $\mathrm{C}$. Coffeen (Coffeen, Anderson \& Associates, Inc., 805 Outlook, Mission, KS 66202)

Because of the physical characteristics of fabric roofs, acoustical problems in structures using such roofs cannot normally be resolved using traditional techniques. This presentation discusses the existing and potential acoustical problems of several existing and proposed facilities that utilize both air supported and tensile fabric roof systems. Also presented will be sound absorbing data for a newly conceived tensile roof system. 
NN5. An evaluation of the acoustics of the O'Connell Center, Gainesville, Florida. Bertram Y. Kinzey, Jr., and Gary Siebein (Department of Architecture, 231 Arch., University of Florida, Gainesville, FL 32611)

The O'Connell Center is a mass seating facility on the University of Florida campus in Gainesville, Florida. Activities housed in this large, air-supported fabric structure range from rock concerts to basketball games to commencement exercises. The study will compare the design methods and programmatic restrictions used by Coffeen, Anderson and Associates, Mission, Kansas, the acoustical consultants for the space, with a post occupancy study including field measurements and user evaluations to understand the ability of acousticians to predict the acoustical performance of this relatively new construction technology. A similar building was constructed at the University of South Florida in Tampa, Florida. A comparison of construction techniques and acoustics between the two structures will also be presented.

3:35

NN6. Acoustical properties of "FABRASORB" acoustical fabrics. John Effenberger (Chemical Fabrics, Bennington, VT)

"FABRASORD" acoustical fabric was developed by CHEMFAB about six years ago to provide a solution to the poor acoustics (U. S. Patent No. 4,152,474) within fabric structures. Based on woven fiberglass and polytetrafluoreotylene (PTFE) resins, it is a natural companion to "SHEERFILL" architectural fabrics which have enabled fabric structures to meet all applicable codes for permanent construction by virtue of the durable and fire-safe properties inherent to fiberglass and PTFE. Key to the successful behavior of a FABRASORB acoustical liner to such construction is close control of the acoustical absorption. This in turn is achieved primarily through control of the porosity of the coated fabric. Data are presented showing sound absorption coefficients over a broad range of frequencies as a function of porosity along with specifications for the noise reduction coefficient achievable.

THURSDAY AFTERNOON, 11 NOVEMBER 1982

SARASOTA ROOM, 1:00 TO 4:30 P.M.

\title{
Session OO. Noise VI: General Noise Control, Measurement, and Analysis
}

\author{
George C. Maling, Jr., Chairman \\ IBM Corp., Acoustics Laboratory, Dept. C18, Bldg. 704, P. O. Box 390, Poughkeepsie, New York 12602
}

Chairman's Introduction-1:00

\section{Contributed Papers}

1:05

OO1. Performance testing of a large reactive silencer. Robert W. Rand and Walter F. Strong (Stone \& Webster Engineering Corporation, P. O. Box 2325, Boston, MA 02107)

The conversion of a $650-\mathrm{MW}$ power station unit from oil to coal included conversion from forced to balanced draft boiler operation and the addition of two induced draft fans rated at over one million CFM each. The fans required $25 \mathrm{~dB}$ tonal noise reduction to meet nondegradation noise criteria. Two tuned reactive/absorptive discharge duct silencers were purchased. Detailed performance tests were required in the purchase specification and were conducted on a prototype silencer to assure the manufacturer's guarantee would be met. Several performance indices were measured: insertion loss, noise reduction, attenuation, and pressure drop. Narrow-band frequency analysis was utilized. Community noise measurements were made following silencer installation. They indicated the silenced fans were inaudible.

$1: 20$

OO2. Noise reduction by a barrier in a small boat. Igor V. Nábð̌lek, Amy $M$. Donahue, and M. Virginia Thompson (Department of Audiology and Speech Pathology, College of Liberal Arts, University of Tennessee, Knoxville, TN 37996-0740)
Noise problems in recreational settings have drawn much less attention than the noise in industry. One source of recreational noise is small boats with outboard motors in which the users are sitting in close proximity to the motor. The levels with A-weighting $\left(L_{\mathrm{A}}\right)$ and without weighting $\left(L_{\mathrm{LIN}}\right)$ reached 94 and $102 \mathrm{~dB}$, respectively, in a $4.5-\mathrm{m}(14 \mathrm{ft})$ boat measured at a distance of $180 \mathrm{~cm}$ from an $85 \mathrm{HP}$ motor and $90 \mathrm{~cm}$ above the floor of the boat with boat speed at $40 \mathrm{~km} / \mathrm{h}$. The noise reduction by four barriers ( 71 and $56 \mathrm{~cm}$ tall, and 1.9 and 0.3 thick) in two different positions was determined. In the lower position, $B$, the barrier was $45 \mathrm{~cm}$ from the motor. In the higher position, $M$, the barrier was raised $10 \mathrm{~cm}$ and moved closer to the motor (to the $15-\mathrm{cm}$ distance). As expected, taller, higher, and thicker boards resulted in greater noise reduction. However, thinner boards showed slightly greater noise reduction at frequencies $2 \mathrm{kHz}$ and above, especially in the $M$ position. Dependent on condition, $L_{\mathrm{A}}$ reduction was between 5 and $12 \mathrm{~dB}$, and the $L_{\text {LIN }}$ reduction was between 0 and 4 dB. In octave frequency bands between 1 and $4 \mathrm{kHz}$, barriers in various conditions produced $11-18-\mathrm{dB}$ noise reduction.

\section{$1: 35$}

OO3. Design of a test station for accurate aerodynamic and acoustic testing of small box fans. Leslie M. Gray (Airflow Research \& Manufacturing Corp., 110 Coolidge Hill Road, Watertown, MA 02172) 
In order to correctly assess the impact of the noise of a box fan in a system, the test apparatus must model correctly the environment mechanisms which affect fan noise. This apparatus must not introduce any unintentional bias in the measurement of fan noise, either in level or frequency structure. This paper reviews the physical mechanisms which cause fan noise, and reviews how these mechanisms are affected by both the fan design, and by the fan environment. Careful consideration of these mechanisms results in a set of acoustic and aerodynamic requirements for measurement of fans. These requirements result in the design of a test station for comparative testing of fans. This design is reviewed, and the benefits compared to other approaches. The paper discusses the limitations of the results that can be expected from these laboratory tests, and proposes a standardized test procedure.

\section{1:50}

004. Noise control of surface mine equipment-A retrofit approach. Leonard C. Marraccini (Mine Safety and Health Administration, Pittsburgh, PA 15213)

Noise problems exist in the mining industry as in other industries Mobile surface equipment such as bulldozers, front end loaders, haulage trucks, and shovels generate noise, that for the most part, exceed current noise regulations. New equipment incorporate noise controls installed by the manufacturer. However, the large percentage of equipment are 5 to 10 years old or older and do not have noise controls installed. Over the last 5 years, a major effort has been put forth to reduce the noise exposure of the operators of these mobile equipment through the use of retrofit noise controls. Results of studies involving flammability of acoustic materials and various installation techniques were incorporated into the work. Results have been very successful with typical noise reductions of 8-12 dBA achieved. Material costs have run typically under $\$ 1000$.

OO5. Application of cepstral technique to remove organ pipe tones from combustion spectra. J. H. Miles and C. A. Wasserbauer. (NASA Lewis Research Center, 21000 Brookpark Road, Cleveland, OH 44135)
Signal reinforcement and cancellations due to standing waves may distort constant bandwidth combustion spectra. Cepstral techniques previously applied to the ground-reflection echo problem [J. H. Miles, J. Acoust. Soc. Am. 61, 35-38 (1977)] are used to obtain smooth broadband data. Internal fluctuating pressure measurements made using a J47 combustor attached to a 6-m-long duct are studied. Measurements made using jet $A$ and hydrogen are compared. The acoustic power levels inferred from the measurements are presented for a range of operating conditions.

\section{2:20}

O06. Comparison of measured and predicted mode scattering associated with spinning mode transmission through a peripherally segmented liner. T. L. Parrott and W. R. Watson (NASA Langley Research Center, Hampton, VA 23665)

An experiment to assess mode scattering in lined circular ducts with peripheral segmentation was conducted. This work is a first attempt to experimentally quantify scattering effects for peripherally segmented liners in a laboratory environment. Mode insertion losses were measured for a six segment, two segment, and uniformly lined duct in the Langley Spinning Mode Synthesizer. Selected spinning modes generated in the synthesizer hardwall source section were allowed to propagate through the lined section into a hardwall duct section with anechoic ternination. Estimated mode insertion losses were obtained from a comparison of transmitted mode amplitudes for the various test liners with those for a hardwall duct configuration. Significant mode scattering was predicted and measured for the two segment liner for incident spinning mode orders of 1 and 3 . For spinning mode order 1 , scattering into spinning mode order 2 was observed. For incident spinning mode order 3, scattering into spinning mode order 4 was observed. For the six-segmented liner, measureable mode scattering was difficult to measure. This was thought to be due in part to inadequate incident mode isolation and to the choice of impedance for the segments.

\section{2:45}

O07. Rough analysis of installation effects on turboprop noise. Paul A. Durbin and John F. Groeneweg(NASA Lewis Research Center, 21000 Brookpark Road, Cleveland, OH 44135)

A rough analysis of noise from a propeller operated at angle of attack, and in the nonuniform flow due to a line vortex approximating a wing flow field suggests installation can significantly affect turboprop noise levels. On one side of the propeller, where the blades approach the horizontal plane from above, decreases of noise occur; while on the other side noise increases. The noise reduction is due to negative interference of steady and unsteady sources. An angle of attack, or distance between propeller and vortex, exists for which noise is a minimum. The relative contributions of thickness and loading noise are discussed, and results are presented both as plots of directivity and as plots of maximum noise level.

\section{3:00}

O08. Peak flat sound pressure level of a sonic boom, with limited frequency response. Robert W. Young (Naval Ocean Systems Center, San Diego, CA 92152)

Peak flat sound pressure (level) at a designated place is a frequently used measure of a sonic boom. Primarily to insure correct measurement of the initial peak of the shock $(N)$ wave, a system flat up to $10 \mathrm{kHz}$ is often specified. Primarily to delineate the waveform in the middle of the $N$ and thus to preserve the duration of the positive pressure phase, a system response flat down to $0.05 \mathrm{~Hz}$ is often specified. The shape of a measured waveform is changed drastically by relatively small changes in the highand low-frequency limitations relative to the duration of the $N$ wave. A measured positive transient can occur after the original $N$ wave, which differs little from the measured initial positive transient. In a single graph, this paper provides an estimate how great an error is made in the measurement of peak sound pressure level of a theoretical $N$ wave, when a system is used whose response is limited by a one-pole high-pass filter and a onepole low-pass filter. As an example for a system with $-3 \mathrm{~dB}$ high-pass response at $8 \mathrm{~Hz}$, and $-3 \mathrm{~dB}$ low-pass response at $1 \mathbf{k H z}$, if the duration of the $N$-wave is $400 \mathrm{~ms}$, the expected error in measured peak sound pressure level is $-0.4 \mathrm{~dB}$; if the $-3 \mathrm{~dB}$ response of the low-pass filter is raised from 1 to $4 \mathrm{kHz}$, the expected error is $-0.1 \mathrm{~dB}$.

\section{3:15}

009. Application of the $4 \times$ real-time digital signal processor for the simulation of programmed sound environment: Aircraft/underwater noise simulation. Jean-Pierre Armand (I.R.C.A.M., 31 rue St. Merri, 75004 Paris, France)

Flight simulation and training programs for underwater detection and identification of ships require the synthesis of realistic sonic environments. The sound synthesis must be accurate, it must be linked to the simulation of motion and of the visual environment, and it must respond to changing internal and external conditions and to the actions of the pilot or operator. Analog sound synthesis techniques cannot meet these severe requirements. Therefore, we have applied the $4 \times$ digital sound processor designed at I.R.C.A.M., to the synthesis of highly realistic aircraft and underwater noises. After analysis of the signals in various situations in frequency and time domains, an acoustical sound generation model is implemented in the processor. The parameters defining the sound characteristics being passed by the host simulation computer, are derived from a mechanical model of the planes or ships. For example, the aircraft noise comprises fan, propeller (if needed), jet, gear, aerodynamic sounds, as well 
as alarms and VHF traffic communications. The underwater noises consist of sea background, biological noise (shrimps), and one boat, the complex noise of the boat itself being made up of diesel engines, gear works, auxiliary equipment, and propeller noise, with various cavitation and blade configurations.

3:30

Q010. Application of the two surface method to determine sound power in-situ in the tobacco industry. Kenneth R. Baki (Gilbert/ Commonwealth, Jackson, MI 49201)

This paper reports the results of tests conducted on tobacco processing and cigarette making machinery using the two surface method for the determination of sound power in-situ. This test method, currently in draft form in the ASTM E33.08D committee, was originally intended for sound power measurement of heavy power plant equipment. Described in this paper is the suitability of this method to measurement of light, high-speed machinery. Also described are the benefits of this method, namely the ability to obtain sound power levels of equipment under typical operating conditions in the environment in which it normally operates. This technique also compensates for the presence of extraneous noise sources as well as the directivity of the machine being studied.

\section{3:45}

O011. Design of a robotic acoustic intensity measurement system. Ivan Morse (Department of Mechanical and Industrial Engineering, University of Cincinnati, Cincinnati, $\mathrm{OH}$ ), P. M. Flanagan, W. J. Atherton (Department of Mechanical Engineering, Cleveland State University, Cleveland, OH), John Coy (NASA, Lewis Research Center, Cleveland, $\mathrm{OH}$ ), and David Stephens (Kodak Corp., Rochester, NY)

A robotic acoustic intensity measurement system (RAIMS) was designed and constructed to automate the analysis of sound fields using acoustic intensity techniques. Key system features include a five degree of freedom manipulator arm, a six element acoustic intensity transducer, a digital control unit for the manipulator, dedicated spectrum analysis and desk top calculator. The manipulator arm was designed for a 45-in. maximum vertical and horizontal reach. The acoustic intensity probe is attached to the robot's wrist. Scanning velocity profiles for the intensity transducer are programmable with a maximum velocity of $5 \mathrm{in.} / \mathrm{s}$ and absolute position information. In addition, the manipulator/acoustic intensity transducer unit weights less than $100 \mathrm{lb}$. The acoustic intensity probe designed for this system helps minimize errors in calculating acoustic intensity by using three pairs of microphones. Preliminary results using this system and future applications will be discussed.

4.00

O012. Correlation of core noise obtained by application of three-signal coherence techniques U. H. von Glahn and E. A. Krejsa (NASA Lewis Research Center, 21000 Brookpark Road, Cleveland,OH 44135]

The prediction of frequency content and noise levels of turbofan engine core noise is re-examined as a result of recent test data and a new diagnostic technique [E. A. Krejsa, NASA TM 82634 (1981)]. The diagnostic technique, utilizing a three-signal coherence method is used to obtain core noise spectra for several engines. Similarities and differences of the spectra are discussed. Finally, the three-signal coherence data are correlated, leading to the development of an improved core noise prediction procedure.

\section{4:15}

O013. Interference effects of neighboring source on acoustic intensity measurements in the nearfleld. G. Krishnappa (Engine Laboratory, National Research Council of Canada, Ottawa, Canada K 1A OR6)

The sound powers estimated from acoustic intensity measurements are subject to local variations depending on the acoustical environment. Interference from the neighboring sources affects intensity measurements. Changes in the sound power levels determined from intensity measurements in the nearfield of a source due to the interference from a neighboring source were examined using two identical ( $320 \mathrm{~mm}$ square) loud speakers. Acoustic intensities were measured in planes parallel to the face of the speakers using the two-microphone technique. The sound power levels measured in front of speaker 1 at close distances was not significantly affected when speaker 2 was generating up to $5 \mathrm{~dB}$ higher sound power levels than speaker 1 . The above experiments were carried out in an anechoic chamber. When the speakers were mounted $1.1 \mathrm{~m}$ from a reflecting floor, the changes in the sound power levels due to the reflections were not significant. Theoretical results of acoustic intensities in the neartield due to the interference of two point monopoles were presented.

THURSDAY AFTERNOON, 11 NOVEMBER 1982

BROWARD AND PALM BEACH ROOMS, 1:00 TO 6:00 P.M.

\author{
Session PP. Underwater Acoustics VI: High-Resolution Sonar
}

\author{
Chester M. McKinney, Chairman \\ Applied Research Labs., University of Texas, P.O. Box 8029, Austin. Texas 78712
}

Chairman's Introduction-1,00

Invited Papers

1:05

PP1. An overview of high-resolution sonar developments. Chester M. McKinney (Applied Research Laboratories, The University of Texas at Austin, Box 8029, Austin, TX 78712)

This overview paper is intended to serve as an introduction to the order papers in this session. It will discuss some definitions of high-resolution sonar in terms of sonar acoustical parameters and in terms of target imaging. Several generic types of sonars (forward scanning, side scanning, etc.) will be described and a brief history of the development of each during the past 40 years will be presented. Various techniques to achieve high cross-range resolution for target imaging will be emphasized. These include large real apertures, synthetic aperture arrays, nonlinear parametric arrays, monopulse techniques, and interferometer arrangements. Techniques for target classification (target size and shape, shadow size and shape, target strength, and resonances) 
will be described briefly. The status of research on target echo structure and the ocean environment in support of the development of high resolution sonar will be summarized briefly. Some of the applications (both naval and civilian) for such sonars will be enumerated.

\section{1:25}

PP2. High-resolution sonars. B. Lallement, N. Clavelloux, Georges Grall, and Jacques Joseph (ThomasCSF, DASM, 29283 Brest Cedex, France)

Submarine search and detail sea-floor studies are problems of a different nature and they do not lead to the same sonar design criteria. High-resolution sonars are the key to an efficient detection and classification of small ground objects, and shadow target imaging reveals to be a very powerful tool. The characteristics of various Thomas-CSF high-resolution sonars are given as examples of implementation of these design principles. Their effectiveness is illustrated by a complete set of pictures obtained at sea by both hull-mounted and towed, side-scan sonar. Finally, different images obtained with a high-resolution sonar simulator are presented to conclude on future trends in this field.

\section{$1: 45$}

PP3. High-resolution sonar system techniques. James Andrews (Naval Ocean Research and Development Activity, NSTL Station, MS 39529)

Advances in sonar hardware and signal processing are having a significant impact on our ability to measure and image the sea floor. These capabilities are in turn rapidly improving our understanding of sea foor morphology and structure and the processes which create and modify them. Side-scan sonars have increased ranges from a few hundred meters to 10 to $30 \mathrm{~km}$. Four systems are of interest in current applications; these are Gloria, Sea Marc, SWATHMAP (SQS-26), and Sea Beam. The information derived from these systems goes beyond simple bathymetric mapping to permit discrimination of sediment types and sea floor roughness. Examples of long-range sonargrams will be discussed in a geologic context to demonstrate these capabilities.

\section{2:05}

PP4. Development of high-resolution side-look sonar. Arthur Nelkin and George A. Gilmour(Westinghouse Oceanic Division, P.O. Box 1488, Annapolis, MD 21404)

About 28 years ago, the Navy had a requirement to scan the bottom of channels and harbors with sufficient resolving power to identify minelike objects on the bottom with cylindrical dimensions 11 in. in diameter by 6 in. long. A side-scan sonar was designed to provide a narrow fan shaped beam focused on a line on the bottom by means of a fixed arc array. The transducer array had to be towed a fixed distance /the radius of curvature of the arc array) off of the bottom for optimum focus. The system scanned the bottom by means of the vehicle movement in the direction of tow and perpendicular to the direction of tow by the travel time of the acoustic signal emitted from the transducer to the bottom. This system had a number of limitations: bottom coverage rate $\left(\mathrm{m}^{2} / \mathrm{h}\right)$, fixed height above the bottom, a limited display capability, and limited signal processing. These led to the development of multibeam arrays with electronic all-range focusing, CRT displays, and adaptive time varied gain tied into the background reverberation levels.

PP5. Hybrid focusing for side-look sonar. George A. Gilmour (Westinghouse Oceanic Division MS 9105, P.O. Box 1488, Annapolis, MD 21404)

Two focusing principles are presently used in high performance $\left(0.1^{\circ}\right.$ beamwidth $)$ side-look sonars (SLS), the mechanical arc, and electronic all-range focusing. Hybrid techniques to combine the two principles are described in this paper. Focusing is partially accomplished using the mechanical arc principle, then completed electronically. This can be thought of as a vernier to allow a mechanical arc SLS to be flown at an altitude other than the radius of curvature. Alternatively, it can be considered to be a way to expand the depth of field of a mechanical arc, or to restrict the depth of field of electronic focusing. The techniques described best fit a very high resolution SLS, e.g., $0.05^{\circ}$ beamwidth, the requires only about three parallel beams per side.

\section{Contributed Papers}

2:45

PP6. Synthetic aperture sonar-Theory and system design. C. D. Loggins (Naval Coastal Systems Center, Panama City, FL 32407)

The synthetic-aperture technique is described and differences between the radar and the sonar implementations of this technique are discussed. Synthetic-aperture beamforming is discussed and compared to beamforming in a conventional (real aperture) sonar. Sonar performance for the synthetic aperture sonar is compared to that of a conventional sonar with respect to area-coverage rate, maximum range, and azimuth resolution.
The vernier technique for increasing area-coverage rate and incoherent averaging for reducing image speckle are also discussed

3:00

PP7. Interferometric array for high-resolution sonars. Georges Grall (Thomson-CSF, DASM, 29283 Brest Cedex, France)

The spatial and temporal properties required by a sonar array to obtain an acoustical picture of the sea floor are reviewed and illustrated by 
simulation techniques. A system using both an interferometer for transmission and a linear array for reception is described. The resolution gain obtained is considered, as well as the system limitations such as angular sector and bandwidth. The comparison with a classical sonar is discussed in detail, and experimental results achieved by this kind of array operating at 200 and $420 \mathrm{kHz}$ are presented.

\section{3:15}

PP8. Odd/even beamformers for high-resolution sonar. T. L. Henderson (Applied Research Laboratory, The University of Texas at Austin, P.O. Box 8029, Austin, TX 78712)

The use of odd/even beamformers is a well-known generalization of the phase comparison and amplitude comparison methods employed in monopulse radars and split beam sonars to locate targets precisely within the mainlobe of the beam pattern. However, it is not well known that target angle can be determined unambiguously, and independently of frequency, from the scale factor that relates the two beam outputs, if the odd/even aperture shadings satisfy the Kerr-Murdock conditions and if the odd beam output has been phase corrected by an ideal integrator. Applications of this technique to high resolution sonar are discussed, emphasizing the use of classical linear regression analysis to compare the outputs. The regression coefficient is used to estimate target angle, and the correlation coefficient gives an indication of the quality of the estimate as a function of time or range. A graphically based method is presented for determining the angular separation of two targets at the same range. The use of scale comparison to measure target angle, rather than channel-tochannel time delay as in split beam sonar, appears to offer several advantages, particularly where broadband signals are involved. [Work supported by NAVSEA.]

\section{3:30}

PP9. Data processing for high-resolution active sonar. R. G. Pridham and C. R. Zentner (Raytheon Company, Submarine Signal Division, Portsmouth, RI 02871)

Active sonars which have high resolution in range and angular sector generate large volumes of data which must be interpreted by the sonar operator. Often the magnitude of the data volume greatly exceeds the capabilities of both modern display technology and the human element. This paper discusses the use of computer-assisted data evaluation and display techniques which address this data mapping/reduction problem. The importance of simulation and real data analysis in studying this problem is explained. Results of a typical simulation are given to show the impact of various performance and computer loading parameters.

\section{3:45}

PP10. Log-periodic acoustic lens-acoustic filter plate study II." Robert L. Sternberg (Office of Naval Research, Boston, MA 02210), Warren A. Anderson (Naval Underwater Systems Center, Newport, RI 02840), Howard F. Ilson, and Owen P. Dickson (Naval Underwater Systems Center, New London, CT 06320)

Graphical experimental data in the form of passive receiving patterns is presented for several high resolution acoustic lens-acoustic filter plate combinations showing the comparative properties of various types of flat and curved filter plates. Experimental data relating the achieved frequency independent constant beamwidth of the lens-filter plate combination to the governing slope parameters of the filter plate are summarized for empirical design purposes. The basic experiments were conducted over the frequency range from 25 to $500 \mathrm{kHz}$ but can be scaled to any band of the corresponding scaled width. [Work supported by ONR and NUSC.] a) Part II of Sternberg, Anderson, and Stevens [J. Acoust. Soc. Am. 63, $1617-1621(1978)]$

\section{4:00}

PP11. A study on FS-PDMP system. Shuying Zhang (Institute of Acoustics, Academia Sinica, Beijing, People's Republic of China)
A signal detection system, which consists of a frequency-stepping signal generator and a corresponding post-detected matching processor, was studied both theoretically and experimentally. The characteristic function determining the detection performance of FS-PDMP system was derived and analyzed, and a set of equipment, which can generate and process an eight-unit long signal with various FS modes $[n](T=8 \tau=200$ $\mathrm{ms}, f n=1360+n \times 40 \mathrm{~Hz}$ ), was made for the experimental studies. Some results are as follows: (1) The output waveforms of PDMP matched with many FS modes were recorded. It was been shown that the output with a narrow mainlobe (the duration $=\tau$ ) and a few side lobes (the level $<p / 8, p$ is the peak of mainlobe) can be obtained from PDMP with the FS modes composed of eight different frequencies and that if there are two or more same frequencies in the FS modes, either the mainlobe will widen or the side-lobe level will increase, or even both. (2) The response of the FSPDMP system to Doppler frequency $\beta$ was investigated. For mode [01234567], the output of PDMP gradually reduces from $p$ to $p / 8$ with the variation of $\beta$ from 0 to $\pm 280 \mathrm{~Hz}$, and the time of output appearance correspondingly changes from 0 to $\pm 7 \tau$. For both modes [75310246] and [05274163], when $\beta$ varies from 0 to $\pm 40 \mathrm{~Hz}$, the reduction of the mainlobe of PDMP output is very fast, and no notable side lobe appears for the former while there are two remarkable ones for the latter. (3) The detection threshold of the FS-PDMP system is about $-8 \mathrm{~dB}$ lower than that of a single unit envelope detector. The above results are in conformity with the theoretical analysis.

\section{$4: 15$}

PP12. Acoustic backscatter from the ocean bottom. T. G. Goldsberry, S. P. Pitt, and R. A. Lamb (Applied Research Laboratories, The University of Texas at Austin, P.O. Box 8029, Austin, TX 78712)

In high-frequency sonar, the dominant interfering noise is virtually always reverberation, and the most important contributor to this reverberation is backscattered energy from the ocean floor. This has been long recognized and in $1964 \mathrm{McKinney}$ and Anderson [3. Acoust. Soc. Am. 36, 158-163 (1964)] summarized the results of a comprehensive experimental investigation of bottom backscattering for a number of shallow water locations. Measurements have recently been made at a shallow water site near San Diego, CA that extend the range of bottom backscattering measurements to smaller grazing angles and narrower beam widths than previously reported. Preliminary results of analysis of these data are described and summarized. [This work was supported by Naval Ocean Research and Development Activity, Code 530.]

\section{4:30}

PP13. The bottom topography survey subsystem (BOTOSS). Hollis Boehme, Roy L. Sullivan, and V. Dan Scott (Applied Research Laboratories, The University of Texas at Austin, P.O. Box 8029, Austin, TX 78712)

The AN/SQN-17 Bottom Topography Survey Subsystem (BOTOSS) is a high-resolution sonar used to obtain precise bathymetric data from which bottom topography charts are made. BOTOSS employs a downward looking, multibeam, pulsed sonar and digital signal processing equipment to acquire, process, and store bathymetric data. BOTOSS is one of four subsystems that comprise the Bathymetric Survey System (BASS) and is functionally interfaced with the other subsystems. The projector and hydrophone arrays are mounted beneath the ship's hull in a Tshaped configuration. The projector array consists of 76 projectors in a support frame located in a streamlined projector dome mounted along the ship's keel. The hydrophone array consists of 128 hydrophones located in a foil assembly athwart the ship's keel forward of the projector dome. Transmit and receive beamforming are computer controlled and independently stabilized against platform pitch and roll through use of ship motion components supplied by the Navigation Subsystem. Other significant components of BOTOSS include control/monitor/interface equipment, a Sperry SP 900 High Speed Signal Processor, Hewlet-Packard HP 2100 computer and peripheral equipment, transmitter equipment, and receiver electronics. The subsystem has been installed and tested on USNS WYMAN (T-AGS 34) and is currently employed in making bathymetric surveys. 
PP14. The Model 706 side-scan mapping system: A marine geophysical survey instrument. Rolland L. Darby (Edo Corp., Western Division, Salt Lake City, UT 84115)

A radically new side-scan mapping system, featuring an evolutionary approach, has integrated multiprocessors for future growth and custom expansion, while maintaining compatibility with proven technology in transducers, towfish bodies, and electrographic chart recorders. The balance between hardware and software solutions has produced a relatively small, modular, easily maintained machine with features that extend beyond the total spectrum of previous side-scan systems. These features include the ability to generate true scale mosaics of the sea floor while simultaneously displaying bathymetric, subbottom, and altitude tracking data on a 19-in.-wide, dry paper, electrographic chart recorder. All processing is performed in real time with additional signal processing possible from magnetic tape during playback. Menu driven user programming simplifies operational use and includes self-test fault diagnosis. Fault tolerant software recovery insures minimum downtime, during surveys. The features and design philosophy are examined in detail followed by a discussion of operational performance data gathered during the last year. Custom enhancement capabilities for specific applications will be covered with details on typical configurations.

\section{5:00}

PP15. High-resolution portable scanning sonar with integral digital display. John M. Low (UDI Group Ltd., Woodside Rd, Bridge of Don, Aberdeen, AB2 8EF, Scotland)

A $500-\mathrm{kHz} 360^{\circ}$ mechanically scanned portable sonar with PPI display is described. The underwater unit [21 $(d) \times 35(h) \mathrm{cm}]$ has a rotating assembly which accomodates two transducers, a transmitting array with a $-3-\mathrm{dB}$ beam of $1.4^{\circ}$ by $27^{\circ}$ and a receiving array of $2.7^{\circ}$ by $27^{\circ}$. The surface control and display unit $[44(w) \times 32(h) \times 4(d) \mathrm{cm}]$ incorporates a standard television format 9-in. display and all controls. Although the sonar is of conventional Ping and Listen analog design, the microprocessor controlled $256 \times 256$ digital display with eight grey levels has considerable advantages over long persistance equivalents typically used in sonars of this type. For example, there can be no blooming or picture fade, display manipulations and expansions can be readily achieved and the output can be recorded on a standard video recorder. Critical to the system design is its ease of operation and interpretation by unskilled operators, leading to a number of new sonar users. Therefore among the applications are civil engineering inspection, underwater archaeological monitoring, seabed debris surveys, wellhead relocation, navigation of free swimming and bottom contact vehicles, seabed scour inspection, etc.

\section{$5: 15$}

PP16. Underwater target echo analysis facility. G. P. Coble (Applied Research Laboratories, The University of Texas, at Austin, P.O. Box 8029, Austin, TX 78712)
A versatile, minicomputer-based acoustic data acquisition and analysis system has been installed at the ARL:UT Lake Travis Test Station. This system differs from analog systems used in the past to measure target strength in that the entire echo is digitized and recorded on 9-track digital magnetic tape, thus retaining both amplitude and phase information. Data recorded on tape are read back into the data acquisition computer for analysis. Data stored in this form are also easily disseminated to other investigators. The echo analysis facility became operational in 1979 and has since been used to study echo characteristics of targets of interest to the U.S. Navy. The system has recently been upgraded to accommodate multiple receiver channel experiments. [Work supported by NAVSEA.]

\section{$5: 30$}

PP17. Effective sensor placement and intersensor processing parameter selection for covergence zone acoustic source localization. Robert Perl (Bolt Beranek and Newman, Inc., 1300 N. 17th St., Arlington, VA 22209) and Robert W. Marshall (Naval Air Development Center, Code 3032, Warminster, PA 18974)

The performance of an intersensor processing algorithm which seeks to localize a moving vessel radiating a narrow-band acoustic signal is dependent upon the placement of the sensors relative to the source, as well as on the cross-correlation algorithm and its processing parameters. Since the localization uncertainty is dependent upon the accuracy of the measurements of intersensor differential time of arrival, the processing bandwidth and coherent integration time of the correlator must be chosen in such a way as to maximize the output signal to noise ratio, while simultaneously avoiding decorrelation effects caused by source-sensor motion and nonidentical ocean transfer function (multipath) effects. In this paper we will demonstrate how a-priori estimates of the acoustic source spectrum, the source kinematics, and the propagation characteristics of the ocean medium were used to specify a reasonable deployment geometry for a set of distributed sensors and an effective set of correlation processing parameters for the over-the-horizon localization of a towed projector. [Work supported by NADC.]

\section{$5: 45$}

PP18. Low grazing angle bottom reverberation measurements at 25, 35, and $45 \mathrm{kHz}$. C. Kenton, E. Moritz, E. Pipkin, S. Richardson, and S. Martin (Naval Coastal Systems Center, Panama City, FL 32407)

The results of measurements, utilizing a mobile platform, of bottom backscattering at grazing angles of $1^{\circ}$ to $30^{\circ}$ are reported. The experiments were carried out in the vicinity of Stage I off Panama City and are comprised of measurements at 25,35 , and $45 \mathrm{kH}$ for $\mathrm{cw}$ and $\mathrm{FM}$ transmitted signals of different pulse lengths. Statistical features of this reverberation data will be discussed as well as comparisons to other bottom reverberation measurements and models. [Work supported by Naval Sea Systems Command.] 
$\div$

\title{
Joint Meeting of Standards Committees S1 and S3
}

The activities of S3 will be discussed first, proceeding to matters of interest to both S3 and S1 and concluding with $\mathrm{Sl}$ activities.

\section{Meeting of Standards Committee S3 on Bioacoustics}

\author{
W. A. Yost, Chairman S3
}

Parmly Hearing Institute, Loyola University of Chicago, 6525 North theridan Road, Chicago, Illinois 60626

Standards Committees S3, Bioacoustics. The current status of standards under preparation will be -ivcussed. In addition to those topics of interest including hearing conversation, noise, dosimeters, hearing aids, etc., consideration will be given to new standards which might be needed over the next few years.

Meeting of Standards Committee S1 on Acoustics

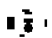

\author{
T. F. W. Embleton, Chairman S1
}

National Research Council, Division of Physics, Montreal Road, Ottawa, Ontario, KIA OSI, Canada

Standards Committee S1, Acoustics. Working group chairpersons will report on their progress in the preparation of standards, methods of measurement and testing, and terminology in physicial acoustics, "telectroacoustics, sonics, ultrasonics, and underwater sound. Work in progress includes measurement of noist 3 burces, noise dosimeters, integrating sound-level meters, and revision and extension of sound level meter specifications. Open discussion of commitee reports is encouraged.

\section{Session QQ. Shock and Vibration IV: Vibration in Structures}

Gerald R. Coonan, Chairman

\section{Peabody Coal Company, P.O. Box 14495, St. Louis, Missouri 63178}

$\cdot \overline{\mathbf{x}}+$

Chairman's Introduction-1:30

1:35

QQ1. System dynamics of large rotating machinery and its foundation. Michael B. de Estrada and ShuhPan Ying (Gilbert/Commonwealth, Jackson, Ml 49201)

System dynamics of a single-speed centrifugal induced draft fan on an isolated pile foundation was investigated to determine the adequacy of foundation design and fan balance. The investigation involved dynamic soils and pile evaluation to establish stiffness and damping of foundation supporting medium. A dynamic computer analysis of complete fan, concrete foundation, soil-pile system was conducted to establish resonant frequencies and system sensitivity to rotating unbalance. Dynamic impact testing using the transfer function technique was performed to determine system natural frequencies and damping for comparison with analytical results. Vibration levels during fan startup and steady-state operation were monitored to determine system resonances, sensitivity, and residual unbalance. Resonant frequencies associated with foundation motion were observed in the 5- to 7-Hz and 10- to 12-Hz regions; and in the the 21- to 25-Hz region for the fan rotor. The fan operating speed is $14.7 \mathrm{~Hz}$ which is outside the resonance zones. The maximum bearing housing sensitivity was measured at 0.027 mils/oz and residual unbalance at $0.0021 \mathrm{lb}$-in.//b which falls within ANSI Standard S2.191975 limits. 
QQ2. Dynamic behavior considerations of floor systems. Patrick W. Linehan (Wiss, Janney, Elstner and Associates, Inc., 330 Pfingsten Rd., Northbrook, IL 60062)

The suitability of a floor system is affected by its dynamic behavior characteristics. The physical properties of the floor system including the stiffness, damping, and natural frequency characteristics are factors which influence the vibration behavior as a result of human (e.g., walking) activity. Further, external forms of excitation (e.g., machinery) adjacent to the floor system can also produce undesirable effects in terms of human response considerations. Wiss, Janney, Elstner and Associates, Inc. have been involved in many programs, including research projects sponsored by the American Iron and Steel Institute, to evaluate the vibration effects of floor systems. Analytical techniques have been developed to calculate the dynamic characteristics, as well as evaluate their behavior from the viewpoint of human response considerations. This presentation will give typical case histories, discuss the breadth of the problem in this area, as well as recommend techniques that can be used to quantify the dynamic characteristics of floor systems including human response considerations.

2:35

QQ3. An overview of vibration problems in fossil power plants. George C. Kao (Sargent \& Lundy Engineers, Chicago, IL 60603)

Vibration problems pertaining to fossil power plant operations are generally recognized in three major areas: flow-induced vibrations, rotary-machine induced vibrations, and operating equipment induced vibrations. Such vibrations have caused failures of structures and equipment and psychological problems to equipment operators. When severe vibrations occurred to operating plants, engineering repairs were implemented often with field vibration data. Several case studies will be used to illustrate sources of vibration and related remedial solutions. Recommendations to minimize such vibration problems are presented.

\section{Contributed Papers}

QQ4. Structural response to low-frequency acoustic excitation. Steven D. Hottman (U.S. Army Construction Engineering Research Laboratory, P.O. Box 4005, Champaign, IL 61820)

Many noise sources at Army installations are of a low-frequency (25 to $125 \mathrm{~Hz}$ ) impulsive nature (e.g., artillery and explosives training, or helicopters). Mitigation of the impacts due to these sources, both on- and offpost, require an understanding of the response of a structure to low-frequency excitation. Little information is available in the literature for response below about $63 \mathrm{~Hz}$. In order to study the response below this band, a structure of typical residential construction has been erected inside the CERL Bi-Axial Shock Test Machine (BSTM) building, and extensively instrumented for vibrational and acoustic response. The BSTM has been utilized to produce impulsive acoustic excitation (114 to $130 \mathrm{~dB}, 25$ to 40 -ms duration) of the structure. A set of specially designed speakers has been utilized to produce nearly pure tone excitation $(90-100 \mathrm{~dB}, 20$ to $125 \mathrm{~Hz}$ ). Production of the excitation signals and preliminary structural response results are presented.

\section{3:20}

QQS. Forced vibrations of two welded plates: A prototype for assessment of the extent of applicability of SEA concepts. Emilios K. Dimitriadis and Allan D. Pierce (School of Mechanical Engineering, Georgia Institute of Technology, Atlanta, GA 30332)

Statistical Energy Analysis (SEA) has as a fundamental tenet that the power flow between two vibrating components of a system is a weighted difference of the energies of the two components, where the coefficients are intrinsic properties of the system, independent of the excitation. A prototype that may give new insight into when and to what extent such a tenet is satisfied for strongly coupled continuous systems is explored here. Two rectangular plates, welded together at right angles along a common edge and simply supported at all other edges are caused to vibrate by random forces applied on one of the plates. The displacement field of each plate is expanded in modal eigenfunctions of the uncoupled plates following a procedure analogous to that previously used for strongly coupled beams by Davies and Wahab [J. Sound Vib. 77, 311-321 (1981)]. The formally exact solution for plate energy spectral density and power flow spectral density is given and is approximated by replacing modal sums by integrals. At higher frequencies and with averages over sufficiently wide frequency bands, the theory yields approximate results consistent with SEA concepts. [Work supported by Whirlpool Corporation.]
3:35

QQ6. Effectiveness of a two-layer damping treatment partially covering a beam. John E. Cole, III (Cambridge Acoustical Associates, Inc., 54 Rindge Ave. Extension, Cambridge, MA 02140)

A damping treatment for an elastic beam consisting of a layer of viscoelastic material beneath an elastic bar is considered. The treatment extends axially over a portion of the beam. Under the assumption of simple end supports and a point force excitation, the vibrational response of the damped structure is analyzed by expanding the beam displacement in a series of normal modes. The normal modes for the bar are taken to be those of a free-free beam and are therefore coupled to each of the simply supported beam modes. A numerical procedure is used to calculate the modal response of the beam from the system of coupled equations. The damping effectiveness of the partial treatment is determined by evaluating the modal loss factor as a function of the extent of coverage. Several equivalent methods are examined to evaluate conveniently the modal loss factor. An efficient numerical scheme is to evaluate the loss factor using the real and imaginary parts of the damped resonance frequency. The results obtained are interpreted with the aid of asymptotic limits for the loss factor of the damped dynamic vibration absorber, and conclusions regarding sensitivity of damping effectiveness to extent of coverage are drawn.

\section{3:50}

QQ7. The effect of longitudinal wave coupling on the diffusion of fexural energy in a one-dimensional waveguide with lossy obstacles. $P$. W. Smith, Jr. (Bolt Beranek and Newman Inc., 10 Moulton St., Cambridge, MA 02138)

Results of a previous power-balance analysis [J. Acoust. Soc. Am. Suppl. 1 62, S83 (1977)] are extended to include flexural-longitudinal coupling at each obstacle. For small coupling, there are two effects. (1) Coupled power constitutes another loss (in addition to dissipation and sound radiation) in determining the attenuation rate ( $\mathrm{dB} /$ obstacle) of the flexural response diffusing from the source. (2) This power reverberates as longitudinal waves and leaks back into flexural, causing a homogeneous background of flexural response in addition to the exponentially attenuated component described in (1). Numerical results are given for a ribbed steel plate underwater. Power-balance methods that postulate highly reverberant flexural fields in each bay (e.g., Statistical Energy Analysis) overestimate the attenuation rate by a factor of about two, in this case. 

Principal results are (1) for small loss factor $\eta(\eta \leqslant 1 / N \pi$, where $N$ is

QQ8. Estimates of power coupling two beams. P. W. Smith, Jr. (Bolt Beranek and Newman Inc., 10 Moulton St., Cambridge, MA 02138)

Davies and Wahab have recently reported extensive precise calculations of the average power coupling the two parts of a beam held by three simple supports, averages being taken over frequency bands and an interval of the ratio of lengths of the two parts [J. Sound Vib. 77, 311 (1981)]. Those results are discussed and compared with prior studies based on one average resonant mode number), the new and old results agree very closely; this is the tightly coupled regime where half the power input to each subsystem is dissipated in the other; (2) agreement is less good for larger $\eta$; in this regime reverberation is slight since round-trip attenuation is large; coupling power varies with the source location within each subsystem; (3) for the particular structure, where the wave energy transmission coefficient of the coupling is large $(0.5)$, there is no regime that is both loosely coupled and highly reverberant.

THURSDAY AFTERNOON, 11 NOVEMBER 1982

ORANGE ROOM, 1:30 TO 5:05 P.M.

\title{
Session RR. Speech Communication VI: Analysis and Synthesis
}

\author{
Edward P. Neuburg, Chairman
}

National Security Agency, Ft. Meade, Maryland 20755

Chairman's Introduction-1:30

\section{Contributed Papers}

\section{1:35}

RR1. Pitch extraction by adaptive window time averaging (AWTA). Joan E. Miller (Bell Laboratories, Murray Hill, NJ 07974)

A running time average applied to a periodic signal will produce a constant, nonvarying output if the averaging interval is equal to the period length or a multiple thereof. Hence, pitch detection may be accomplished by finding the averaging interval which minimizes the variation in the output and taking the reciprocal of this time interval as the fundamental frequency. This notion has been implemented for the extraction of pitch from speech signals. Some important features of the techniques are (1) time integration, an inherent aspect of this method, contributes to the robustness of its performance, (2) the variance of the averaged signal may be normalized, which facilitates a voiced-unvoiced decision, and (3) crucial parameters of the analysis can be easily adapted to the current pitch estimate for better results in sections of changing period length. Details of the procedure will be described, and results obtained for a variety of speech samples will be shown.

\section{1:49}

RR2. On reducing pitch sensitivity of $L P C$ parameters. Sharad Singhal and Bishnu S. Atal (Acoustics Research Department, Bell Laboratories, Murray Hill, NJ 07974)

Mean-squared prediction error criterion used in linear predictive coding of speech has a number of inherent shortcomings. For low pitch frequencies, the LPC analysis largely ignores the pitch-related fine structure of the speech spectrum. However, the sensitivity of the LPC parameters increases rapidly as a function of the pitch frequency. This sensitivity can be directly traced to the mean-squared prediction error criterion used in LPC analysis. Moreover, in the presence of noise and errors resulting from the assumption of an all-pole model, the minimization of prediction error leads to a perceptually suboptimal solution. In this paper, the LPC analysis is considered as a short-time spectral envelope matching problem. Using the LPC-derived parameters as initial values, a search procedure is used to refine the LPC parameter estimates. The new procedure minimizes a perceptual distance metric between the spectrum based on LPC parameters and the samples of the speech spectrum at spectral peaks.
2:03

RR3. Performance evaluation of a real-time LPC coding technique. N. Rao Vemula and Phil T. McLaughlin (General Instrument, Microelectronics, $600 \mathrm{~W}$. John Street, Hicksville, NY 11802)

There are a number of LPC-based speech synthesis integrated circuits available on the market today. For many practical applications of these chips, it is advantageous to utilize a real-time code generation system. Traditional LPC analysis techniques, such as covariance, autocorrelation, and PARCOR, process blocks of speech samples yielding one set of LPC parameters for each block. Autocorrelation and covariance techniques require matrix inversion and are too complex for hardware implementation. PARCOR-type lattice techniques require a large amount of data storage at each stage of the lattice. In this study we have evaluated a fast and efficient LPC analysis technique suitable for hardware implementation for real-time operation. Unlike the traditional LPC analysis techniques, the real-time technique processes individual samples of speech. The LPC parameters are first initialized and then updated as each sample is processed. Computer simulation has shown that the real-time technique yields reasonable spectra. Computer generated synthetic sounds from the real-time technique have almost the same quality as those generated from the block-data techniques. Sounds synthesized using the real-time technique on our SP0256 chip are indistinguishable from those synthesized using traditional techniques.

\section{2:17}

RR4. Test of an automatic syllable peak detector. M. Fleck (Yale University, New Haven, CT and Bell Laboratories, Murray Hill, NJ 07974) and M. Y. Liberman (Bell Laboratories, Murray Hill, NJ 07974)

It is a venerable and commonplace intuition that syllables are "peaks of sonority." Previous work by Mermelstein [J. Acoust. Soc. Am. 58, 880883 (1975)] suggests that this intuition is nearly true on the acoustic surface of speech, and that relatively crude algorithms based on amplitude variation can work fairly well at counting syllables and locating their approximate extent. We have devised a simple speaker-independent algorithm for detecting sonority peaks in continuous speech, and tested its accuracy as a syllable finder on several paragraphs of casual reading by four speakers. Performance was encouraging: less than $10 \%$ misses, and 
less than $1 \%$ false triggers. The misses were about evenly divided among (1) vowel-vowel transition, where no sonority minimum is expected, (2) intervocalic sonorants with insufficient amplitude dip, and (3) devoiced or deleted schwas. Methods for dealing with these cases will be discussed.

RR5. Articulatory constraints on vocal tract area functions and their acoustic implications. S. J. Butler and H. Wakita (Speech Technology Laboratory, 3888 State St., Santa Barbara, CA 93105)

A mathematical model for generating vocal tract area functions for vowel-like sounds has been developed. Using computer simulation of a transmission line analog of the vocal tract, the relationship between acoustic features (in this case formants) and parameters of the model (i.e., articulatory parameters) has been investigated in order to develop improved algorithms for acoustic-articulatory and articulatory-acoustic transformations. The model has been found to be a useful tool for study of acoustic phonetics, examination of the applicability of speaker normalization techniques, and for the refinement of high-quality speech synthesis. Extensions of the model to include consonantal vocal tract configurations are also presented.

\section{2:45}

RR6. The dialogue terminal. Ron Sorace (Acoustics Research Department, Bell Laboratories, Murray Hill, NJ 07974)

The dialogue terminal is a direct application of research in speech recognition, synthesis, and coding at Bell Laboratories. It represents a rather novel approach to state-of-the-art interactions between man and machine and has potential for a variety of applications. Current speech coding techniques permit integration of voice and data for digital tranmission and, coupled with high density memory circuits, enable storage of large amounts of speech for playback, synthesis, or recognition. Speech storage and editing and rate modification are necessary for applications such as voice-mail. Speech synthesis capability such as formant or speechfrom-text techniques are available and have sufficient quality to provide a degree of intelligibility which is adequate for output of data and issuing spoken prompts. The terminal is designed to perform isolated word recognition since this method represents the state-of-the-art. However, for the more experienced user the capability to parse a continuous sequence of commands will give the terminal the ability to adapt to the experience level of the user. The resulting mode of operation consists of synthetic voice prompts and voice commands in a dialogue format.

\section{2:59}

RR7. Synthesis of continuous speech by concatenation of isolated words. Mark A. Randolph and Victor W. Zue (Room 36-541, Research Laboratory of Electronics, Massachusetts Institute of Technology, Cambridge, MA 02139)

This paper reports a feasibility study of synthesizing continuous speech by concatentation of modified isolated word templates. Continuous speech obtained simply by word template concatenation has several inherent problems. It cannot account for the prosodic features normally found in continuous speech, nor can it account for coarticulation; the natural transitions that occur at word boundaries. In an effort to alleviate the first problems, the synthesizer is provided with information describing the duration, the fundamental frequency $(f 0)$ contour, and the energy contour of each sentence. The synthesizer draws from a dictionary of word templates each stored as a sequence of LPC parameters. Before concatenation, the time scales of the synthesis templates are nonlinearly warped using the alignment path obtained from a level building connected speech recognition algorithm. In addition, the $f 0$ and energy contours of the word templates are modified. To account for coarticulation, the various parameters are smoothed at word boundaries. The goal of this synthesis system is an output information rate of 200 bits per second of speech. A demonstration tape will be played.
RR8. Implementation of a prosody scheme in a constructive synthesis environment. Kathleen $M$. Goudie, Kun-Shan Lin, and Gene A. Frantz (Consumer Products Group, Texas Instruments, P.O. Box 10508, M.S. 5893, Lubbock, TX 79408)

In a constructive synthesis environment, lack of natural prosody detracts both from the naturalness and from the intelligibility of synthetic speech. It is time consuming and frustrating for the linguistically untrained user of a constructive-synthesis system to assign pitch patterns and unit durations by mere guesswork. A semi-automatic prosody assignment scheme has been designed here which takes the majority of the prosody-assignment burden off the user and which is capable of assigning fairly natural-sounding pitch patterns to a constructive synthesis string of allophones. The user is required only to mark the primary and secondary stress locations in the text or in the allophone string, plus an indication of whether the constructed phrase should rise or fall in pitch at the end. These stress marks serve as anchor points for the system to compute both an intonation contour and a timing-adjustment contour for the whole phrase. Thus, with a minimum of effort and linguistic knowledge, the user may achieve a relatively natural-sounding constructive speech phrase.

\section{$3: 27$}

RR9. Speech synthesis using allophones. Janet G. May and Eugene H. Lee (General Instrument Corporation, Microelectronics Division, 600 W. John Street, Hicksville, NY 11802)

Synthesizing speech by concatenating allophones is a new application of linear predictive coding (LPC) that provides an unlimited vocabulary. Since the stored units are individual speech sounds, any English word can be synthesized by concatenating the appropriate sounds. Our inventory contains 59 speech sounds and five pauses. Each allophone was created by extracting it from the digital waveform of a word, and then synthesizing it. The sounds are called allophones because some phonemes have two or three versions for different environments. For example, $/ \mathrm{k} /$ and $/ \mathrm{g} /$ each have three allophones: one to be used before front vowels, one before back vowels, and one in final position. In contrast, research revealed that the same $/ p /$ can be used in all environments. Isolating final $/ r /$ from preceding vowels was impossible because of important transitional information. Therefore there are five phonemically unique $V+/ r /$ combinations. Due to the omission of many transitions, allophone synthesis suffers from reduced intelligibility when compared with the technique of synthesizing words as units. This compromise seems minor, however, when its flexibility and low cost are considered.

\section{3:41}

RR10. Formant synthesis: Technique to account for source/tract interaction. J. J. Yea and D. G. Childers (Department of Electrical Engineering, University of Florida, Gainesville, FL 32611)

Research in speech synthesis is presently concerned with methods for improving intelligibility and naturalness. But most work to date has dealt with intelligibility, i.e., the synthesized speech is intelligible but monotone and machinelike in quality. Although this type of speech is useful in some applications, the users of speech synthesizers are demanding a more natural sounding synthesized speech. We are addressing this issue in our research. In particular we are studying the influence of the glottal source function on the production of synthesized speech. Our data base consists of inverse filtered speech, ultra-high-speed laryngeal films, and the electroglottograph waveforms, all temporally synchronized in our experiments. We use the experimentally derived source waveforms obtained from these various method as the excitation for a serial/parallel Klatt formant synthesizer to synthesize sentences. We then evaluate the naturalness of this synthetic speech by listening tests. Our goal is to rank order the contribution of different source parameters to the naturalness of synthetic speech. More specifically, we report on our results to date of the effect of source-tract interaction upon the production of natural sounding speech. 
RR11. Coding of fundamental frequency at low rates. D. O'Shaughnessy (INRS Telecommunications, Bell Northern Research, 3 Place du Commerce, Nuns Island, Quebec H3E 1H6, Canada)

Speech coding at low rates usually requires the coding of excitation information such as fundamental frequency $(F 0)$. Typically, $F 0$ is calculated every $10-25 \mathrm{~ms}$ and represented using $5-6$ bits. Thus $200-600 \mathrm{bits} / \mathrm{s}$ are dedicated to excitation. Since $F 0$ does not change radically between consecutive voiced frames, a differential coding scheme can partially reduce the $F 0$ rate without perceptual degradation in the synthetic speech. However, even lower rates can be achieved by approximating the $F 0$ patterns as linear rises and falls. Each such change is coded as a pair of $F 0$ values and a duration. Specifically, each peak or valley in a smoothed $F 0$ contour is coded, as well as the elapsed time since the previous point. Typical English utterances (not including pauses) appear to average seven peaks and valleys per second. If the durations are coded using 4 bits/sample, $F O$ contours would occupy less than $70 \mathrm{bits} / \mathrm{s}$ with little degradation in speech quality. Such a reduction in $F 0$ storage rate could be useful in very low rate vocoders.

4:09

RR12. Real-time determination of multi-phase excitation for LPC speech synthesis. R. J. Hanson, B. S. Atal, and J. Kohut (Acoustics Research Department, Bell Laboratories, Murray Hill, NJ 07974)

We describe a real-time implementation of a multi-phase excited LPC speech coder using Bell Laboratories DSP chips. A major advantage of multi-pulse excitation is that it produces natural-sounding synthetic speech and avoids both pitch analysis and voiced-voiceless decision. Pulse amplitudes and locations are determined by a noniterative, analysis-bysynthesis procedure which minimizes a perceptual distance criterion. The multi-pulse analysis is performed in fixed-point arithmetic on two DSPs. The analysis yields four excitation pulses for every $5 \mathrm{~ms}$ of speech, which are necessary for producing high quality synthetic speech. A companion real-time LPC area-parameter analyzer using DSP chips was described previously by Hanson and Olive [J. Acoust. Soc. Am. Suppl. 1 69, S18 (1981)].

\section{$4: 23$}

RR13. A speech data base facility using a computer-controlled cassette tape deck. K. L. Shipley, A. E. Rosenberg, and D. E. Bock (Acoustics Research Department, Bell Laboratories, Murray Hill, NJ 07974)

A speech data base collection facility is described which uses a cassette tape deck interfaced to a laboratory computer for storing and accessing speech samples. The computer, under program control, has access to the transport functions as well as the position counter of the cassette deck. In addition, there is a capability of reading and writing digital headers. Digital headers are particularly useful in enabling descriptive information to be written directly with the speech samples as well as enabling precise location of individual samples in a data base. The facility offers an attrac- tive means for compactly storing large data bases of speech samples with relatively easy access to individual samples. An experiment is described in which speech sample utterances were digitized and processed on-line simultaneously with recording directly onto cassette tapes with descriptive digital headers. The recorded speech samples were digitized and processed in the same way and compared with the directly digitized and processed samples. No significant degredation was obtained attributable to the analog cassette recording.

RR14. Intelligibility of running speech using a tactile vocoder aided by lipreading. P. L. Brooks, B. J. Frost, K. Chung, and J. L. Mason (Department of Psychology, Queen's University, Kingston, Ontario, K7L 3N6, Canada)

In a previous paper [P. L. Scilley and B. J. Frost, J. Acoust. Soc. Am. Suppl. 169, S123(1981)] we reported that an artificially deafened subject could identify 150 live spoken words using only the in formation obtained through a tactile vocoder. The vocoder consists of $16,1 / 3$-octave filters whose outputs, after detection and logarithmic amplification, are transmitted to a 16-channel linear solenoid array. The subject continued word learning at approximately the same rate until 250 words were identified. Presently, the subject's performance is being evaluated using (a) phrases derived from the 250 -word vocabulary (b) novel words, and (c) novel phrases comprised of frequently occurring English words. This material is in an "open set" format with each stimulus presented only once so the subject has no idea of the stimulus possibilities. With no additional training after the word learning experiments the subject could understand and correctly repeat $81 \%$ of the (a) phrases, $75 \%$ of the (b) words, and $75 \%$ of the (c) phrases using the tactile vocoder plus lipreading. In the lipreading alone condition $59 \%$ of (a) phrases, $40 \%$ of (b) words, and $48 \%$ of the (c) phrases were repeated correctly. [Work supported by National Health and Welfare Canada.]

\section{$4: 51$}

RR15. Evaluation of a model for generating and displaying synthetic animated lip shapes. Allen A. Montgomery (Army Audiology and Speech Center, Walter Reed Army Medical Center, Washington, DC 20012)

This paper summarizes the development of the capability to synthesize on a vector-driven graphics system the lips of a talker producing phrase length utterances. Details of the algorithm for generating smoothly varying sequence of images using a phoneme string as input are given, including methods for controlling timing and specifying form and extent of coarticulation. Also, data on the intelligibility of real and synthetic utterances are presented and compared. The model seems to account satisfactorily for changes in lipshape due to variation in phonetic context and rate of speaking, and the implementation of the model is shown to produce patterns of intelligibility close to those obtained with natural talkers. [Work supported by Department of Clinical Investigation, WRAMC.] 


\title{
Session SS. Engineering Acoustics IV: Measurement Techniques
}

\author{
Bernard E. McTaggart, Jr., Chairman
}

Naval Sea Systems Command, 14509 Bellemeade Lane, Germantown, Maryland 20767

Chairman's Introduction-2:00

\section{Contributed Papers}

2:05

SS1. Calibration of a dynamic pressure transducer using reciprocity in a plane-wave resonator. G. W. Swift, A. Migliori, J. C. Wheatley (Group P-10, MS-M764 Los Alamos National Laboratory, Los Alamos, NM 87545), and S. L. Garrett (Department of Physics and Chemistry, Naval Postgraduate School, Monterey, CA 93940)

Using the principle of reciprocity in a plane-wave resonator we have obtained on accurate absolute calibration of a dynamic pressure transducer in 10-bar helium gas. The resonator consisted of a 365-cm-long, 7.3crn-diam stainless steel tube with ordinary electrodynamic loudspeakers sealed to both ends. The transducer to be calibrated, mounted near one end of the resonator, was a silicon strain-gauge piezoresistive bridge with a nearly frequency-independent sensitivity from zero frequency through $400 \mathrm{~Hz}$. We found that the reciprocity measurements agreed with a static calibration of the transducer using a mercury manometer to within $1 \%$. consistent with instrumental uncertainties. [Work supported by the U.S. Department of Energy.]

\section{2:20}

SS2. Displacement measurements of vibrating transducers. W. Jack Hughes (Applied Research Laboratory/The Pennsylvania State University, P. O. Box 30, State College, PA 16801)

An in-air measurement procedure has been developed to measure the displacement of a vibrating surface. Specifically the amplitude of vibration has been measured for the radiating surfaces of several transducers (primarily tonpilz type) of different designs. The objective being to determine to what degree the radiating head of a transducer acts as a rigid piston at resonance. In some cases it was known prior to measurement that the radiating head was vibrating with a modal structure, i.e., the comers vibrated out of phase with the center. A fiber optic measurement system was used in order to perform noncontact vibration displacement measurements. The usable frequency range is dc to $100 \mathrm{kHz}$. Displacement amplitudes on the order of one micron have been measured accurately. The apparatus, which was built at the Applied Research Laboratory at The Pennyslvania State University, allows displacement mapping of the entire surface of a transducer's radiating head. [Work supported by NAVSEA.]

\section{2:35}

SS3. Sound intensity probe calibration. Gunnar Rasmussen (Brüel \& Kjær, 18 Nærum Hovedgade, 2850 Nærum, Denmark)

Calibration methods and data for sound intensity probes using two microphone technique will be discussed. Choice of configuration and data to be expected for a given configuration is important for the frequency range and the accuracy. Calibration aecuracy overall, in the high-frequency range and in the low-frequency range, will be discussed along with recommendation regarding the choice of microphone type. Especially the problems associated with the ANSI standardized method of using grazing incidence for pressure measurements will limit the performance of a two microphone sound intensity probe due to diffraction and scattering phen- omena. A configuration is proposed which will minimize problems with diffraction, scatter, rotational symmetry, and phase shift and maintain a well defined spacing $\Delta r$ between the two microphones, and at the same time allow multiple choice of different spacings $\Delta r$.

\section{2:50}

SS4. Directivity pattern measurements of a transducer array in a highly reflective enclosure. Gary A. Zets (Central Acoustics Laboratory, University of Cape Town, University Private Bag, Rondebosch 7700, Republic of South Africa)

Underwater acoustic measurements are commonly made in laboratory test tanks because of the advantages offered by a controlled environment. Violations of some of the conditions assumed by theory often occur, making direct comparisons between measurement and theory impossible unless specialized techniques are utilized. In particular, directivity pattem measurements of highly directional transducers require good freefield and farfield conditions which are usually unattainable in a test tank. In this paper, directivity patterns of a directional array were measured in a highly reflective enclosure to determine the best agreement with theory under these severe conditions. For convenience, and without loss of generality, the experiment was conducted in air. A linear 11 -element microphone array was constructed and tested in a reverberation chamber using a computer controlled, gated measurement system. The theoretical array directivity pattern was computed under both ideal and real conditions and comparisons showed that extremely good measurements can be made if the correct procedure is followed.

\section{3:05}

SS5. A computer-controlled high pressure hydrophone calibrator. Robert E. Crooks and James M. Powers (Naval Underwater Systems Center, New London, CT 06320)

A computer-controlled calibration method was developed which measures the quasistatic receiving sensitivity of piezoelectric hydrophones over a 0 to $7 \mathrm{MPa}$ pressure range. In this method, a pressure step is applied to the hydrophone and the resulting change in charge is measured. This procedure is repeated over the desired pressure range and a plot hydrophone sensitivity versus pressure is generated. Hydrophones up to $10 \times 10 \times 30 \mathrm{~cm}$ in size can be calibrated. A microcomputer was used to handle the large number of repetitive measurements and to perform arithmetic operations, graphic display, and data storage. [Work supported by NAVSEA code 63R.]

\section{3:20}

SS6. Microphone placement factors for one-half inch diameter mierophones. John P. Seller (U.S. Department of Labor, Mine Safety and Health Administration, 4800 Forbes Avenue, Pittsburgh, PA 15213)

A series of tests were conducted to compare the measurement of an undisturbed acoustical field with a 1/2-in.-diam microphone to the measurement of the aocustical sound with a 1/2-in.-diam, shoulder mounted, 
man-worn microphone. One-third octave band spectrum measurements were made and thè positional-center head microphone placement factor was deternined for thret shoulder positions, and five microphone orientations. The acoustical tests were performed in a reverberation room and in an anechoic chamber on five test subjects. The free field measurements were conducted for azimuthal angles from $0^{\circ}-360^{\circ}$ in increments of $45^{\circ}$. Averaged microphone placement factors are presented for a $1 / 2$-in.-diam microphone, in one-third octave bands, from 200 to $8000 \mathrm{~Hz}$, for all test locations, microphone orientations, and acoustical environments. Where possible comparisons are made between the results of this study and, earlier work which determined the microphone placement factors for a 1-in.diam microphone. A computation of the best microphone location orientation tas made for lour defined types of noise spectra. This was done in order to predict the optimum position for the location of a noise dosimeter microphone under free and diffuse field conditions.

\section{3:35}

SS7. Dielectric losses in a large signal hydrophone far from resbnance evaluated from its hysteresis. Yariv Porat and Margaret E. Browder (Naval Research Laboratory, Underwater Sound Reference Detachment, P. O. Box 8337, Orlăndo, FL 32856)

A large signal hydrophone may deviate from the linear dielectric behavior which is characterized by the hysteresis cuive. If the hydrophone is operated far frort its mechanical resonance, the only losses evident in the hysteresis loop will be dielectric. This work describes a method to calculate the hysteresis pattern using Landau phenomenological theory [F. Jona and G. Shirane, Ferroelectric Crystals (McMillan, New York, 1962)] and network analysis of a Tower Sawyer's bridge. The algorithm has the Landau free energy coefficients, the physical dimensions of the hydrophone, and the presumed lossy elements of the network as data. An iteration method was developed for calculating the blocked capacitance of the hydrophone to less than $1 \%$ convergence. The method also enables one to predict the temperature and pressure dependence of losses from Landau theory. The algorithm is programmed on a HP9815S desk calculator and plotted for comparison with the oscilloscope recorded pattern of the hysteresis.

\section{3:50}

S58. Simulation of an underwater loiad for transducers using a lossy polytheric layer. B. H. Nicholls (Plessy Marine Rescarch Unit, Wilkinthroop House, Templecombe, Somerset, England)

The availability of in air test facilities for sonar arrays, particularly regarding validatiơn procedures for the drive amplifiers of the arrays, offers a convenient and cost effective approach for carrying out these tests. An investigation, based on the use of lossy polymers normally employed in anechoic applications, has identified a material with the appropriate properties. This results in a matched impedance with the required loss parameters to enable a close simulation of a water load. The study was considertd, initially, from single transducer element characteristics and developed further ds an array configuration dummy load. The design philosophy and salient features of a specific system are discussed and features of the test procedure are highlighted to demonstrate a unique approach to material characterization.

\section{5}

SS9. The 3-3 parameters for piezoelectric ceramics: New parameterimeasurement relations and transducer design indications. Gordon E. Maltin (Martin Acoustics Software Technology, 2627 Burgener Blvd., San Diego, CA 92110) and Jeffrey L. Johson (Naval Ocean Systems Center, San Diego, CA 92152).

Many underwater acoustic transducers are designed to utilize the 3-3 mode of piezoelectric ceramic materials. The dominant elastic transducer parameter for such segments and stacks is $s_{33}^{D}$ [G. E. Martin, J. Acoust. Soc. Am. 35, 510-520 (1963); 36, 1496-1506 (1964)]. Usually each segment is an annular ring and the direct measurement of $s_{13}^{0}$ is not considered valid. This paper describes parameter evaluation techniques for such 3-3 parameters based upon finite element analyses of a short, thick annulus with relatively large cross section. The measured thickness resonance and antiresonance frequencies are related to the parameters using the equations for a long 3-3 bar with correction factors. The correction for $s_{33}^{D}$ is small and for $k_{33}$ it is nearly $10 \%$. Experimental results for the ring for $20^{\circ}-120^{\circ} \mathrm{C}$ showed that $s_{33}^{D}$ is relatively constant whereas dielectric constant and coupling coufficient $k_{\mathbf{3 3}}$ are strongly dependent upon ambient temperature. Transducer design and reliability implications due to these 3-3 parameter results are discussed. Prior parameter-measurement relations are reviewed and compared to analyses here based upon finiteelement models. [Work supported by NAVSEA 63X.]

\section{$4: 20$}

SS10. Computation of the sound field near baffied and unbaffled planar sources using the fast Fourier transform. Earl G. Williams (Naval Research Laboratory, Code 5133 Washington DC 20375)

The fast Fourier transform is used to evaluate Rayleigh's formula and the resulting bias errors are carefully analyzed. The unacceptably large errors which often occur are nearly completely eliminated by the dse of an "integrated" Green function to replace the usual Neumann Green function in the Rayleigh integral. This new Green function is shown to cause a large spatial attenuation of the replicated sources and to provide highly accurate results. Furthermore, it is shown that FFT techniques can be used to obtain not only the nearfield pressure, but also the nearfield vector velocity and vector intensity for a planar source of arbitrary shape (with specified surface velocity). An iterative technique is presented using the FFT to evaluate the radiation from unbaffled planar sources. In particular the computation of the pressure on the surface of vibrating, unbaffled disk is shown to be in excellent agreement with the exact solution uifng oblate spheroidal functions. The radiated fields from unbaffled sources of any geometry and surface velocity may be evaluated using this technique. The FFT provides an extremely fast and economical numerical solution for radiation problems, especially if an array processor is used to speed the calculations. Solutions using the iterative technique with an array processor are estimated to take only in the order of 10's of seconds to compute 16000 field points.

\section{4:35}

SS11. Measurement of phase of the surface reflection coefficient using two harmonically related signils. Frank Tighe, Jr. (Transducer and Acoustics Subsystems Design Engineering, Westinghouse Electric Corporation, Oceanic Division, Annapolis, MD 21404)

A method for measuring the phase of the surface reflection coefficient using two harmonically related signals was developed and experimentally verfied on samples of high and low loss elastomer and foam materials. This method utilized the property that two harmonically related signals maintain a constant relative phase when propagating over identical paths in a nondispersive medium. When both signals were reflected simultaneously and then detected, the phase angle of the complex surface reflection coefficient was determined by comparing the two reflected signals. The theoretical study resulted in equations which show the reflection coefficient to be a function of frequency and of material properties (sound velocity, density, and absorption) of a medium and a reflecting or boundary surface. Underwater acoustic experiments were conducted using reflected collinear, harmonic acoustic signals to analyze sample material with measured parameters comparing closely with predicted values. [Work from author's Ph.D. dissertation, Catholic University 1982 by same title.]

\section{4:50}

SS12. Computerized measurement and tracking of acoustical resonances. D. Conte and S. L. Garrett (Department of Physics, Naval Postgraduate School, Monterey, CA 93940)

A system will be described which incorporates a Hewlett-Packard 85 "desk-top" computer to control a frequency synthesizer and read the output of a lock-in analyzer to measure, display and record the resonant frequencies, amplitudes, and quality factors of several modes of an acous- 
tical resonator. The system is capable of locating, measuring, and tracking the resonant modes as parameters which effect sound speed and attenuation are varied. [This work has been supported by a grant from the NPS Foundation Research Program.]

\section{5;05}

SS13. Low cost digital data acquisition system for measuring the free decay of standing waves in continuous media. Roger $W$. Meredith (Old Dominion University Research Foundation, P. O. Box 6369, Norfolk, VA 23508) and Allan J. Zuckerwar (NASA Langley Research Center, Hampton, VA 23665)
A low-cost digital system based on an 8-bit Apple II microcomputer has been designed to provide on-line control, data acquisition, and evaluation of sound absorption measurements in gases. The measurements are conducted in a resonant tube, in which an acoustical standing wave is excited, the excitation removed, and the sound absorption evaluated from the free decay envelope. The free decay is initiated from the computer keyboard, after the standing wave is established and the microphone response signal is the source of the analog signal for the A/D converter. The acquisitilon software is written in assembly language and the evaluation software in BasIC. This presentation will discribe the acoustical measurement, hardwhre, software, and system performance. Also, measurements of sound absorption in air will be presented as an example. [Work supported by NASA under Grant NAG 1-234.]

\author{
Session TT. Musical Acoustics IV: General Session \\ 4: \\ Gabriel Weinreich, Chairman \\ Randall Laboratory of Physics, University of Michigan, Ann Arbor, Michigan 48109
}

Chairman's Introduction-2:00

\title{
Contributed Papers
}

\section{2:05}

\section{TT1. Modal coupling in vibrating plates. Graham Caldersmith thd Thomas D. Rossing (Departinent of Physics, Northern Illinois University, DeKalb, IL 60115)}

In square isotropic plates, some of the degenerate mode pairs are split in frequency due to coupling between longitudinal and transverse strain (which might be called Poisson coupling), and the same splitting of degenerate modes may occur in rectangular orthotropic plates having a certain length/width ratio. We have studied this coupling in free plates of aluminum and spruce (quarter-cut and skew-cut) and determined Poisson's ratios from the frequencer ratio of the $(2,0 \pm 0,2)$ modes (called the $X$ and ring modes by Luthiers). The critical length/width ratio was found to be considerably greater $(3)$ the skew-cut spruce than in the quarter-cut spruce (2.1), due to the smaller cross-graip"elastic modulus and Poisson ratio. a) Permanent address: 48 Denn is fy, Latham, ACT 2615, Australia.

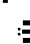

2:20

TT2. Modal analysis and its application. Gordon Ebbitt, Hideo Suzuki, and Lou Abbagnaro (CBS Technology Center, 227 High Ridge Road, Stamford, CT 06905)

Modern modal analysis techniques provide a method for the rapid determination of structural mode shapes and frequencies. This analysis utilizes the impulse measurement of the transfer inertance (acceleration/ force) between a reference point and points on the surface of a structure. The procedure involved in the analysis will be outlined and potential problems in its implementation will be discussed. These potential problems include the improper location of the reference position, the number of measurement points chosen, the resolution of the processing system, apd the spectral content of the impulse. The application of modal analysistio musical instruments will be considered and some results will be presented.

\section{2:35}

TT3. Experimental study of vibration and radiation characteristics of a violin. William Y. Strong, Jr., Emil Torick (CBS Technology Center,
227 High Ridge Road, hamford, CT 06905), and Julian Maynard (Department of Physics and Applied Research Laboratory, 104 Davey Laboratory, Pennsylvania State University, University Park, PA 16802)

A study of a violin's vibration and radiation characteristics has been undertaken using two techniques. The goals of the study are to perform a modal analysis of the instrument and to use the nearfield acoustical holography (NAH) system located at the Pennsylvania State University to reconstruct the instrument's radiated sound field. The modal analysis will locate the various resonances of the top and back plates and show the relative motion of the plates. The NAH system will locate those areas on the instrument that prodice the energy that radiates to the farfield. This paper will discuss the work completed to date and present the results obtained from this work.

$m$

\section{2:50}

TT4. The "4X" real-time digital signal processor. Peppino DiGiugno, Jean Kott, and Jean-Pierre Armand (I.R.C.A.M., 31 rue St. Merri, 75004 Paris, France)

We have realized a real-time digital signal processor for applications in real-time analysis and synthesis of sound and 4 -The $4 \mathrm{X}$ processor works as a peripheral connected to a host comput, 1 main feature of the $4 \mathrm{X}$ processor is its modularity. It consists of up to 16 elementary processors working in parallel. Each of these processors contains a data memory, an address memory, a microprogram memory, a function memory, a multiplier, and an arithmetic and logic unit. All the computations are done in 24-bit fixed point arithmetic. The $4 \mathrm{X}$ processor also contains 256 programmable clocks and a large dual buffer memory for recording and reproduction of signals to and from the host computer disk. The programming of the $4 X$, which is done in high level languages (FORTRAN and $C$ ), allows an easy programmation of the different possible structures of the machine, as oscillators, envelope generators, filters and so on. These capabilities make it possible to implement all known audio synthesis and processing techniques. The $4 \mathrm{X}$ processor is now used for musical composition, synthesis of aircraft noise for flight simulators, synthesis of underwater noise of submarines, audio mixing, and psychoacoustic stimulus generation. 
UU2. Scattering of ultrasonic waves in the MHz range from rough surfaces and rough defects in metals. G. Quentin, M. de Billy, A. Jungman, and F. Cohen Tenoudji (G.P.S. University Paris 7, 2 Place Jussieu, 75251 Paris, Cedex 05, France)

A review is presented on the study of the scattering of ultrasonic waves from water-metal interfaces. These surfaces are either random, or periodically rough. For random rough surface the inverse problem concerns the determination of the rms roughness, the autocorrelation length of the surface's profile and the probability density function of heights. Comparisons will be shown with theoretical scattering diagrams. For periodic surfaces we use ultrasonic spectroscopy in order to study the frequency dependence of the diffracted waves. At normal incidence "anomalies" are observed in the spectra and are explained by mode conversion to waves propagating along the surface. A comparison is made between experimental and theoretical spectra. The inverse problem is also discussed. We shall also describe experiments made on a rough penny-shaped crack embedded in a titanium block and show that one can ultrasonically "measure" not only the size of the crack, but its roughness and the autocorrelation length of its profile.

\section{3:05}

UU3. Scattering by a periodic rough surface. Richard K. Johnson (Schlumberger-Doll Research, P.O. Box 307, Ridgefield, CT 06877)

An exact theory based on the extended boundary conditions method [S. L. Chuang and R. K. Johnson, J. Acoust. Soc. Am. 71, 1368-1376 (1982)] for acoustical scattering from a periodic rough interface between a fluid and a solid is reviewed. Good agreement between theoretical calculations and laboratory measurements is demonstrated. Theory and measurements are used to explore the effects of roughness height and shape as well as material properties on the scattering behavior. Interesting phenomena involving surface wave generation and finite aperture transducer effects are also discussed.

UU4. The boss model for reflection and scattering by rough surfaces. Victor Twersky (Department of Mathematics, University of Illinois, Chicago, IL 60680)

This review emphasizes the effects of including pair correlations and dense packing on coherent reflection and incoherent scattering of sound by distributions of protuberances (bosses) on rigid or free base planes. The earlier energy conserving forms for the specularly reflected wave, the surface impedance, and the differential scattering cross section per unit area, are obtained in terms of a transform of the scattering amplitude of an isolated boss. Low-frequency approximations are developed to delineate the roles of the packing density and multipole coupling effects, and explicit results are given for semi-elliptic cylinders and hemi-ellipsoids. It is shown that for lossless bosses, the reflection coefficient has a minimum, and the incoherent scattering a maximum, at the packing density corresponding to maximum fluctuations in the number of bosses per unit area. It is also shown that multipole coupling effects may be misinterpreted in data inversion programs as changes in boss shape; if such effects are not accounted for, then, e.g., hemispheres may be mistaken for hemiellipsoids broadened along the base plane and shortened along the normal. Illustrations include problems of the gaseous boss adhering to a rigid bottom.

4:05

UU5. Scattering by surfaces with multiple and infinite hierarchies of roughness scales. Elan Moritz (Naval Coastal Systems Center, Panama City, FL 32407)

The theories of scattering from surfaces with multiple roughness scales and surfaces with an infinite hierarchy of roughness scales are reviewed. The statistics of scattered waves are developed from the statistics of the rough surface for single frequency, narrow-band and broadband signals. The influence of variations of ratios of dominant (wavelength) scales will be explored for rough surfaces with finite multiplicity.

\section{Contributed Papers}

4:35

UU6. Scattering from statistically rough surfaces: A 3-D numerical study of the Kirchhoff approximation. David H. Berman and John S. Perkins (Acoustics Division, Code 5160, Naval Research Laboratory, Washington, DC 20375)

The angular spread of acoustic radiation scattered from a rough surface is computed using the Kirchhoff approximation. Both the dependence on azimuth and elevation of the scattered intensity are plotted for model surface statistics. Results are compared to those of perturbation theory and to those of an extended Kirchhoff approximation which formally reduces to the perturbation results in the small surface height limit. In all cases studied, the perturbation result for the scattered intensity near the specular direction dominates the Kirchhoff result. Both hard and soft surfaces are considered and an example of non-Gaussian statistics is presented. The Kirchhoff integral is also compared to an approximation of that integral proposed by M. V. Berry [J. Phys. A 8, 566-584 (1975)].

UU7. Interaction of ultrasonic waves by periodically rough liquid-solid interfaces. L. Adler, A. Jungman (Department of Welding Engineering, Ohio State University, Columbus, $\mathrm{OH} 43210$ ), J. M. Claeys, and O. Leroy (Katholieke Universiteit Leuven Afd, Kortrijk 8500, Belgium)

When a broadband ultrasonic wave interacts with periodically rough liquid-solid interfaces at normal incidence the reflected spectrum contains several minima which are characteristics of the various parameters 
of the interface as reported earlier [J. Acoust. Soc. Am. Suppl. 168, \$81 (1980)]. An analytical approach to this problem will be discussed which uses general boundary conditions suggested by Poiree [Rev. Cethedec 39, 21-24 (1974); 48, 7-34 (1976)]. These boundary conditions are expressed in terms of continuity of functions involving velocity field $v$, and stress tensor $T_{i j}$ in liquid and solid, respectively, as: $\mathbf{V}^{\mathbf{1}} \cdot \nabla \phi=\mathbf{V}^{2} \cdot \nabla \phi$ and $\Sigma_{j=1}^{3} T_{i j}^{1}(\nabla \phi)_{j}=\Sigma_{j=1}^{3} T_{i j}^{2}(\nabla \phi)_{j}$, where $\phi(x, z)=f(x)-z$ defines the profile. The frequency dependence of the reflection coefficient is calculated for various surface parameters. Correlation between height of the irregularities and intensities of diffracted orders is shown to depend mainly on the characteristics of the solid. Theoretical results for the reflected spectra are in very good agreement with experiments. [Work partially supported by the National Foundation of Belgium.]

\section{5;05}

UU8. Low-frequency scattering of an impulse by a cylindrically corrugated surface at grazing incidence. Francois Jouailleca) and Herman Medwin (Department of Physics, Naval Postgraduate School, Monterey, CA 93940)

Point source radiation at grazing incidence to a rough surface generates a slightly dispersive Biot boundary wave. When the surface is a series of adjacent circular half-cylinders (radius a), the boundary wave is frequency, range, and azimuth dependent. Its characteristics have been mea- sured at low frequencies $(K a<1)$ in an anechoic chamber model to a range of $2.2 \mathrm{~m}$ and from 2 to $20 \mathrm{kHz}$. The results agree generally well with the theory [I. Tolstoy, J. Acoust. Soc. Am. 72, 960-972 (1982)]. [Work supported by the Office of Naval Research.] ") Permanent address: D.R.E.T., 75998 Paris Armees, France.

UU9. Scattering from two and three gratings of densely packed compliant tubes. Ronald P. Radlinski and Robert S. Janus (Naval Underwater Systems Center, New London, CT 06320)

In previous studies [R. P. Radlinski and M. M. Simon, J. Acoust. Soc. Am. 72, 607-614 (1982)] of scattering from two gratings of compliant tubes, excitation of higher order antisymmetric modes was shown to degrade insertion loss performance. In this presentation, scattering from gratings which are densely packed with tubes is considered for both two and three layer configurations. For these cases, additional types of structural modes must be incorporated into the analysis. The low-frequency scattering from the multiple gratings can be understood in terms of equivalent circuits. The restricted benefits from the use of three gratings are discussed in terms of the parameters which control the frequencies of transmission maxima. Calculations will be compared with experimental data. [Work supported by NAVSEA.]

\title{
Session VV. Physical Acoustics VII: Elastic and Thermal Effects; Measurement Methods and Devices
}

\author{
Anthony J. Rudgers, Chairman \\ Naval Research Laboratory-USRD, P.O. Box 8337, Orlando, Florida 32856
}

Chairman's Introduction-2:00

Contributed Papers

\section{2:05}

VV1. Resonance modes of an elongated chamber in the presence of a temperature gradient. N. Jacobi, J. Robey, and E. Trinh (Jet Propulsion Laboratory, California Institute of Technology, Pasadena, CA 91109)

The Helmholtz equation was solved analytically for a step temperature profile, so as to describe measurements earlier reported [E. Trinh, T. G. Wang, and J. Robey, J. Acoust. Soc. Am. Suppl. 1 70, $\$ 90$ (1981)] for an elongated vertical chamber with a significant temperature gradient along its main axis. The solution is expressed in terms of trigonometric and/or hyperbolic functions, and a dispersion, or characteristic, equation is obtained for the resonance frequency of the compound system by imposing appropriate boundary conditions. It is found that the hot region sustains periodic, standing wave solutions in some cases, and exponentially decaying, evanescent wave solutions when the standing wave cannot propagate from the cold to the hot region. The characteristic equation is solved by numerical iterations, resulting in temperature-dependent pressure profiles and resonance frequencies which are in good qualitative agreement with experimental results. The frequency spectrum for a given temperature gradient is also computed by searching for the roots of the characteristic equation. The simple step model is extended to describe a gradual temperature change, and for a linear dependence the solutions are expressed in terms of Airy functions. [Work supported by NASA.]

\section{2:20}

VV2. Acoustic energy transfer in the presence of a temperature gradient. M. Barmatz, J. Allen, and M. Gaspar (Jet Propulsion Laboratory, California Institute of Technology, Pasadena, CA 91 109)

The transmission of acoustic energy through a temperature discontinuity region of a long tube resonator has been investigated. The temperature discontinuity divides the tube into two homogeneous regions. With a constant velocity generator at the cold end, the condition for maximum power transform to the termination load at the hot end is that the source transducer see as high an impedance as possible. The boundary condition at the temperature discontinuity is that the interface impedance be the same as seen from the hot or cold region. For the case of negligible attenuation, this condition will be met if the characteristic impedance in each region $(p c / s)$ is the same. For example, in the case of the interface impedance being zero, the lengths of the two regions must be odd multiples of $\lambda$ / 4 , while if this interface impedance is infinite, the lengths must be integral 
multiples of $\lambda / 2$. The effects of non-negligible attenuation on the energy transfer will also be presented. Experiments are now underway to test the predictions of this model. [Work supported by NASA.]

\section{$2: 35$}

VV3. Analytic expressions for resonant and anti-resonant frequencies in a composite viscoelastic material. Ronald L. Kligman and Walter Madigosky (Naval Surface Weapons Center, White Oak, Silver Spring, MD 20910)

General analytic representations for resonances and anti-resonances in the effective dilatation modulus and effective density have been found. These are valid in the Rayleigh regime $k_{1} a<1$, where $k_{1}$ is the compressional wavenumber in the host medium and $a$ is the radius of the spherical inclusion. The resonance frequency with respect to the dilatation modulus can assume values that are real or purely imaginary which correspond to the cases of an underdamped and overdamped system, respectively. A similar situation holds true for the case of the anti-resonance frequency. The effective density shows resonant and anti-resonant behavior analogous to the effective dilatation modulus. All these frequencies are shown to be functions of the weighted ratio of the Lamé constants of the host and inclusion media. Some specific examples will be considered.

\subsection{0}

VV4. Dynamic elastic constant determination via measurement of the acoustic field scattered by a spherical elastomer. Jean C. Piquette (Nava) Research Laboratory, P. O. Box 8337, Orlando, FL 32806)

Dynamic elastic constants can be determined by solving the inverse scattering problem of an infinite plane acoustic wave incident upon a spherically shaped elastomer. A least-squares analysis is used to find the values of the elastic constants that are most consistent with measured scattered pressures. The approximate values of the elastic constants required to initiate the least-squares procedure can be calculated from expressions that have been developed which constitute approximate relationships between these elastic constants and the first few coefficients of the series expansion for the scattered pressure. An experimental investigation of this technique is being developed. [Work supported by NAVSEA 63R.]

\section{3:05}

VV5. Thermoviscous effects on acoustic scattering by elastic solid cylinders and spheres. Wen $\mathrm{H}$. Lin and A. C. Raptis (Components Technology Division (308), Argonne National Laboratory, Argonne, IL 60439)

This paper presents analytic solutions and numerical results of the scattering of plane sound waves from a thermoelastic circular cylinder and from a thermoelastic sphere in an infinite, thermoviscous fluid medium. The thermoelastic properties of the cylinder and the sphere and the viscosity and thermal conductivity of the surrounding fluid are taken into consideration in the solutions of the acoustic-scattering problems. The associated acoustic quantities, such as the acoustic-radiation force, the acoustic attenuation, and the acoustic-scattering pattern, are first derived in closed forms and then evaluated numerically for a given set of material properties. Numerical results show that increasing fluid viscosity tends to increase the magnitudes of the acoustic-radiation force, the acoustic attenuation, and the angular distribution of the scattering pattern. However, the opposite trends are observed when the fluid temperature increases. The plots of the acoustic attenuation coefficients versus the dimensionless wavenumber of the incident sound wave reveal oscillatory phenomena, which are caused by the resonant vibrations of the cylinder or the sphere.

\section{$3: 20$}

VV6. The acoustic radiation force on a heated (or cooled) rigid sphere. Theory. C. P. Lee and T. G. Wang (Jet Propulsion Laboratory, California Institute of Technology, 4800 Oak Grove Drive, Pasadena, CA 91109)
A finite amplitude sound wave can exert a radiation force on an object due to the nonlinearity of the wave field [L. V. King, Proc. R. Soc. (London) Ser. A 147, 212-240 (1934)]. In this work we study the radiation force on a rigid small sphere (i.e., in the long wavelength limit), which temperature is different from that of the environment, in the absence of gravity. This investigation is relevant to material processing in space using acoustic levitation. We first calculate the temperature-induced perturbation of the incident wave plus the wave scattered from the sphere surface using Bonn approximation. Then we evaluate the radiation force on the sphere using the time-averaged integral momentum equation. Both isotropic and nonisotropic temperature profiles are considered. In this calculation the acoustic effect and heat transfer process are essentially decoupled because of the long wavelength limit. The heat transfer information required for determining the force is contained in the parameters which are integrals over the temperature distribution. [Work supported by NASA.]

\section{3:35}

VV7. Design of a traveling lens using acoustic solitons. P. P. Banerjee and A. Korpel (Department of Electrical \& Computer Engineering, The University of Iowa, Iowa City, IA 52242)

In an acoustic traveling wave lens device (ATWL) a high amplitude sound pulse acts to focus an incident light beam which tracks the moving pulse. By this method the resolution of acousto-optic beam deflectors may be increased by an order of magnitude. If a liquid medium is used for maximum effect, the power of the lens is limited by nonlinear behavior, which eventually leads to shock formation. In principle this may be avoided if the medium could be made dispersive such that the phase velocity would decrease with increasing frequency. In that case the pulse shape should remain constant due to a balance between nonlinear and dispersive effects. Such pulses are called solitons; they have, of late, been the subject of much research but, with the exception of optical solitons in fibers, have not been investigated in an applications context. In this communication we discuss a possible application of acoustic solitons. We first describe their properties relative to the nonlinear and dispersive parameters of the medium. We then propose a method of obtaining the necessary dispersion by making the rigid walls of a sound tank locally reacting through the use of thin plastic strips. [This research is sponsored in part by the National Science Foundation under Grant \#ECS 8121781.]

\section{3:50}

VV8. The use of an array receiver in baffle performance measurements. R. Maple (Naval Underwater Systems Center, New London, CT 06320) and D. A. Sachs (Bolt Beranek and Newman Inc., 50 Moulton Street, Cambridge, MA 02238)

The use of an array receiver in place of the omniphone traditionally employed in baffle performance measurements can reduce panel edge diffraction interference as well as provide a measurement which averages over any field nonuniformities caused by panel inhomogeneities (e.g., tile edges and vacuum channels). In addition, boundary reflections and ambient noise can be suppressed. In this paper, data obtained from some recent experiments employing a planar array as a receiver is discussed. The predictions of theoretical models for baffle performance and panel edge diffraction are compared with the experimental results. [Work supported by NUSC, New London, CT.]

\section{4:05}

VV9. Image formation by multiple shading. C. H. Jones, J. H. Thompson, and J. W. Kesner (Westinghouse Oceanic Division, P.O. Box 1488, Annapolis, MD 21404)

A novel method for obtaining an improved image from an array of hydrophones is described. The technique involves generating a set of different images. These images are then combined using an appropriate algorithm. The composite image is superior to any one of the original images. The technique achieves good angular resolution with low spurious side lobes. Three examples are given for a 16-element array using computer simulation. The procedure is applicable to line arrays, planar arrays, and conformal arrays. 
VV10. Acoustic loading in planar networks. M. El-Raheb and P. Wagner (Applied Mechanics, Jet Propulsion Laboratory, California Institute of Technology, Pasadena, CA 91109)

The acoustic pressure in complex planar networks is determined by a method which adopts Green's function surface elements. The boundary of each duct is discretized into small straight and circular elements each carrying a source density of constant strength. This process enables us to convert the Fredholm integral equation in the source density into a set of linear algebraic equations. Increase in computational efficiency is attained using the transfer matrix technique. Basically, the influence coefficients including elements on the interface of conjoined ducts are determined separately for each duct in the network. The unknown source density vector on the walls is expressed in terms of the source density vector at the junction lines. Enforcing compatibility of acoustic pressure and velocity at the interface of consecutive ducts determines a system of linear algebraic equations in the form of tridiagonal blocks. The transfer matrix approach is best suited for long configurations formed by repeated patterns of straight, circular, and branched ducts. The computation of transfer matrices is then limited to a few basic building segments of the network.

\section{$4: 35$}

VV11. A finite difference solution for the propagation of sound in a variable area duct. S. I. Hariharan (ICASE) and H. C. Lester (ANRD) (NASA Langley Research Center, Hampton, VA 23665)

In this paper an explicit time/space finite difference procedure is used to simulate the propagation of sound in a quasi one-dimensional duct containing high Mach number subsonic flow. Nonlinear acoustic equations are derived by perturbing the time-dependent Euler equations about a steady compressible flow. The governing difference relations are based on a fourth-order, two-step (predictor-corrector) MacCormack scheme. Difference equations for the source and termination boundary conditions are derived from the appropriate characteristic relationship. The solution algorithm functions by switching on a time harmonic source and allowing the difference equations to iterate to a steady state. A significant advantage with this approach is that the nonlinear terms can be retained and evaluated without incurring additional computer cost over and above that required for a linear model calculation. The numerical procedure is applied to a Crocco-Tsien duct with a 2.0 area reduction ratio. Throat Mach numbers up to 0.95 are computed for source levels of $135 \mathrm{~dB}$. Overall spatial rms distributions are computed by temporal averaging and SPL levels of the harmonics are computed by finite Fourier transforms. Linear results are validated by comparison with the results of a Galerkin/weighted-residual finite element program. Nonlinear results are compared with experimental data.
VV12. The free-field two-microphone method for the measurement of the acoustic impedance of the ground. Constantine Glaretas ${ }^{a)}$ (Pennsylvania State University, Noise Control Laboratory, University Park, PA 16802)

A new method for measuring the acoustic impedance of the ground has been developed. The free-field two-microphone method, as it is called, consists of measuring the sound pressure field, radiating from a loudspeaker source and propagating parallel to the ground, by two microphones located on the same vertical axis. The transfer function between these two microphone signals is shown to be, for total coherence, equal to the ratio of velocity potentials, predicted by the appropriate point-topoint propagation theory over a plane boundary. A numerical search with a digital computer provides the value of ground impedance which satisfies the transfer function relationship stated above. Two ground models have been tested: a locally reacting and an extended reacting model. From the close agreement of local and extended reaction models in predicting ground impedance, it is concluded that the grass-covered surfaces tested here can be considered as locally reacting, without significant error. The free-field two-microphone method presents certain advantages over other impedance techniques. These advantages include a reduction in possible errors resulting from uncertainties in the exact location of ground surface, and the potential to explore the acoustic properties of the ground using different ground models. " Present address: Wyle Laboratories, 128 Maryland St., El Segundo, CA 90245.

\section{5:05}

VV13. Computer-controlled measurement of dynamic compressibility of polymers. A. W. Nolle and T. S. Lin (Applied Research Laboratories and Department of Physics, The University of Texas at Austin, Austin, TX 78712)

Some years ago a method for measuring the complex compressibility of polymers was reported from the National Bureau of Standards [J. E. McKinney, S. Edelman, and R. S. Marvin, J. Appl. Phys. 27, 425-430 (1956)], and used in several comprehensive studies. The sample is in a rigid chamber small compared to desired wavelengths, along with two piezoelectric transducers and a liquid under controlled static pressure. To find compressibility, one needs the complex transmission factor for this system, that with a standard sample, and that for liquid only. We have adapted the experiment to computer control. The transmission factor is read repeatedly from a precision two-channel lockin detector during a single 100 -min temperature scan while recurrent 100 -s frequency scans occur. A smaller chamber permits measurements above $10 \mathrm{kHz}$. The reduction procedure smooths the data and determines the compressibility for specified standard frequencies and temperatures. Results are shown for a polyurethane for $-2^{\circ}$ to $30^{\circ} \mathrm{C}$ and 0.4 to $11 \mathrm{kHz}$. [Supported by ONR. Assistance of ARL, Penn State is also acknowledged.] 


\title{
Session WW. Psychological Acoustics V: Discrimination: Time, Intensity, and Frequency
}

\author{
David S. Emmerich, Chairman
}

\author{
Department of Psychology, State University of New York at Stony Brook, Stony Brook, New York 11794
}

Chairman's Introduction-2:00

\section{Contributed Papers}

2:05

WW1. Detection of a temporal gap as a function of level and frequency. Søren Buus (Auditory Perception Laboratory, 4l3MU, Northeastern University, Boston, MA 02115) and Mary Florentine (Communications Research Laboratory, 133FR, Northeastern University, Boston, MA 02115)

The minimum detectable gap, MDG, of an octave band of noise was measured as as function of frequency and level. Seven frequencies ranging from $0.25-14 \mathrm{kHz}$ were tested. For each frequency, the level of the noise varied from about $10 \mathrm{~dB}$ SL to a spectrum level of $47 \mathrm{~dB}$ SPL. The gap was produced by turning off and on the signal with a rise-fall time of $1 \mathrm{~ms}$. To eliminate the effect of spectral splatter, each octave band of noise was presented with its complementary band-stop masker. The masker was continuous and its spectrum level was equal to that of the signal. The MDG was measured with a modified BUDTIF procedure and a 2I, 2AFC paradigm. Results for three normal listeners show that the MDG decreases at all frequencies with increasing level up to a spectrum level of $30-40 \mathrm{~dB}$ SPL. For example, the MDGs at $4 \mathrm{kHz}$ were about $9 \mathrm{~ms}$ at $3 \mathrm{~dB}$ SPL (spectrum level), about $6 \mathrm{~ms}$ at $33 \mathrm{~dB}$, and about $7.5 \mathrm{~ms}$ at $53 \mathrm{~dB}$. The effect of frequency is much more pronounced: the MDG at the best level decreases from about $40 \mathrm{~ms}$ at $250 \mathrm{~Hz}$ to about $5 \mathrm{~ms}$ at $4 \mathrm{kHz}$ and above. These results indicate that the auditory filter may limit performance at frequencies below $4 \mathrm{kHz}$, whereas a different mechanism seems to dominate at frequencies above $4 \mathrm{kHz}$. [Work supported by NIH grant RR07143 and Northeastern University's Research and Scholarship Development Fund.]

\section{2:20}

WW2, Duration discrimination of noise bursts presented at a rapid rate. Brian R. Shelton (Department of Psychology, University of Western Ontario, London, Ontario N6A 5C2, Canada)

The influence of the presentation rate of two noise bursts on the discrimination of duration was assessed using an adaptive two-alternative, forced-choice procedure. The time difference required for reliable discrimination was determined for $70 \mathrm{~dB}$ SPL noise bursts of 25-, 50-, 100-, $200-, 400-, 800-$, and 1600 -ms duration. The interval between the presentation of the two noise bursts in each trial was set at either 50,200 , or 1600 ms. The Weber fraction, $\Delta t / t$, was determined by an interaction between the noise duration and the rate of presentation. With a presentation interval of $1600 \mathrm{~ms}, \Delta t / t$ was a constant of 0.08 at all noise durations. Presentation intervals of 50 and $200 \mathrm{~ms}$ produced $\Delta t / t$ functions which were elevated for short noise durations, but converged on the $0.08 \Delta t / t$ performance level with noise duration of $1600 \mathrm{~ms}$. The $\Delta t / t$ for a $25-\mathrm{ms}$ signal was 0.30 with a 50 -ms presentation interval, and 0.22 with $200 \mathrm{~ms}$ between noise bursts. The results are discussed in relation to neural constraints on the timing of rapidly presented stimuli. [Work supported by NSERC.]

\section{2:35}

Ww3. Intensity discrimination at high frequencies in the presence of noise. Neal F. Viemeister (Department of Psychology, University of Minnesota, Minneapolis, MN 55455)
Previous experiments on intensity discrimination in the presence of band-reject noise indicate that recruitment of unsaturated fibers via spread-of-excitation along the cochlear partition is not necessary for the maintenance of the large dynamic range of human hearing. The present experiment is similar to that of Viemeister [J. Acoust. Soc. Am. 56, 1594 1600 (1974)] but extends the situation to frequencies for which an intensity code based upon temporal fine structure is presumably not possible. The primary result of this experiment is that Weber's Law holds over at least a $75-\mathrm{dB}$ range for intensity diserimination of $200-\mathrm{ms}$, diotic bursts of bandpass noise $(6-14 \mathrm{kHz})$ in the presence of a relatively intense, complementary band-reject noise. This indicates that neither large-scale spread-ofexcitation nor temporal synchrony is necessary for the auditory system to maintain its dynamic range. [Supported by NIH NS 12125.]

\section{2:50}

WW4. Can the opposite ear provide profile information? David M. Green and Gerald Kidd, Jr. (Laboratory of Psychophysics, Harvard University, 33 Kirkland Street, Cambridge, MA 02138)

Consider a spectrum produced by 21 components, all equal in amplitude and spaced in equal logarithmic steps in frequency. (The frequency ratio between successive components being 1.12, or about 6 tones/oct.) The signal is an increment in the middle component, near $950 \mathrm{~Hz}$. A twoalternative, forced-choice method is used to measure the signal threshold. The overall level of the spectrum varies randomly within trials by \pm 20 dB. We have described the detection process as profile analysis since it amounts to detecting a "bump" in an otherwise flat spectrum of uncertain overall level. For practiced observers the threshold for the signal, added in phase to the $950-\mathrm{Hz}$ component, is about $14 \mathrm{~dB}$ below the level of that component (a Weber fraction of about 0.2 ). In this experiment we determine the threshold for the increment in the $950-\mathrm{Hz}$ component when the other 20 components are eliminated from the test ear and are presented to the opposite ear. The signal threshold increases by about $12 \mathrm{~dB}$ (a Weber fraction of approximately 1.0). Apparently, the information in the opposite ear cannot be effectively integrated with the signal component to create a profile. [Work supported by NIH.]

\section{3:05}

WW5. Profile analysis for irregular intensity spectra. Gerald Kidd, Jr. and David M. Green (Laboratory of Psychophysics, Harvard University, 33 Kirkland Street, Cambridge, MA 02138)

In our previous experiments with the simultaneous spectral discrimination process called profile analysis, we measured the just detectable increment in the center component of a multitone complex. In those experiments, the multitone background was uniform in level. The present paper describes the results from conditions where the center component of the complex (the one to which the signal was added) was varied in level relative to the other components, thus forming a "pedestal" on the otherwise flat spectrum. The pedestal was varied from $18 \mathrm{~dB}$ below to $24 \mathrm{~dB}$ above the level of the other background components. Four equilog multitone backgrounds were used consisting of $3,5,11$, or 21 components including the pedestal component. The overall level of the sound was varied $\pm 20 \mathrm{~dB}$ within trials. The threshold of an increment in the middle component of the complex was measured as a function of the pedestal 
level. The resulting function had a rather broad, shallow "U" shape. The minimum in the function usually occurred at a small positive pedestal value. In keeping with our earlier findings, the more components in the background, the lower the increment threshold. An asymmetry was observed between positive and negative pedestal levels that may be attributed to masking. [Work supported by NIH.]

\section{3:20}

WW6. Discrimination of modulation depth of SAM noise. Gregory H. Wakefield and Neal V. Viemeister (Department of Psychology, University of Minnesota, Minneapolis, MN 55455)

Difference limens (DLs) for modulation depth of sinusoidally amplitude modulated (SAM) wideband noise were measured as a function of the modulation depth of the standard, the frequency of modulation $(25,100,400 \mathrm{~Hz})$, and the spectrum level of the noise $(0,20,40 \mathrm{~dB}$ SPL). Preliminary results for one subject show that psychometric functions are linear and parallel in $\log d^{\prime}$ and $10 \log \left(m_{1}^{2}-m_{0}^{2}\right)$, where $m_{1}$ and $m_{0}$ are the modulation depths of the variable and standard stimuli, respectively. In the main study, three subjects were tested using a 2IFC adaptive procedure. The $\mathrm{DL}\left[10 \mathrm{Log}\left(m_{1}^{2}-m_{0}^{2}\right)\right]$ is at, or slightly above, the modulation detection threshold when $m_{0}$ is small. For $m_{0}$ above modulation threshold, the DL is a positively accelerating function of $20 \mathrm{Log}\left(m_{0}\right)$. Weber's Law does not describe the discrimination of modulation depth over any appreciable range. Across all conditions, DLs tend to converge to values around $-9 \mathrm{~dB}$ when the level of the standard is $-5 \mathrm{~dB}\left(m_{0}=56 \%\right)$ and generally cannot be measured beyond this level. The effects of noise level on the DL are, in general, small. [Supported by NIH grant NS12125 and by USPHS HD0715I traineeship from the Center for Research in Human Learning.]

\section{3:35}

ww7. Monaural phase effects: Release from masking. Manfred R. Schroeder (Drittes Physikalisches Institut, University of Göttingen, Federal Republic of Germany and Bell Laboratories, Murray Hill, NJ 07974), S. Mehrgardt, and H. W. Strube (Drittes Physikalisches Institut, University of Göttingen, Federal Republic of Germany)

Masked thresholds of tones, masked by multicomponent harmonic stimuli, depend critically on the phase spectrum of the masker. Monaural phase effects in excess of $40 \mathrm{~dB}$ (release from masking) are reported. Based on these results, intelligible speech has been created by phase manipulation of harmonic signals having a time-invariant flat spectrum.

\section{$3+50$}

WW8. Formant discrimination and speech intelligibility at low signal-tonoise ratios in subjects with a sensory-neural hearing loss. J. W. Horst and R. J. Ritsma (Institute of Audiology, University Hospital Groningen, Postbox 30.001, 9700 RB Groningen, The Netherlands)

Formant discrimination experiments were performed with a group of hearing-impaired subjects who according to an audiological diagnosis had a purely sensory-neural hearing loss. The subjects were selected for their relatively flat audiograms. Most of these subjects showed abnormal susceptibility to ambient noise with regard to speech intelligibility. Apart from discrimination in noise of signals with a formantlike spectrum, the frequency selectivity as measured by means of psychophysical tuning curves and speech intelligibility in noise was measured. Significant correlations were found between formant discrimination in noise, frequency selectivity, and speech intelligibility in noise.

4:05

WW9. Auditory signal processing in hearing-impaired persons with residual high-frequency hearing. Glenis $\mathbf{R}$. Long and John $\mathbf{K}$. Cullen, Jr. (Kresge Hearing Research Laboratory of the South, Department of Otorhinolaryngology, LSU Medical Center, New Orleans, LA 70119)
A number of persons with severe sensorineural hearing losses in the frequency range below $6000 \mathrm{~Hz}$ have been shown to have near-normal thresholds for frequencies above $6000 \mathrm{~Hz}$. This paper reports on measures of high-frequency signal processing in seven persons with this type of hearing impairment. Measures taken include estimates of difference limens for intensity and frequency, gap detection, rate discrimination, and narrow-band masking functions. Although little normative data exist for comparative purposes, measures of frequency, intensity, and time resolution are within the range of what one would anticipate by extrapolation. Intensity and frequency differences limens, and wideband and narrowband masking functions also were obtained in the region of impaired hearing (i.e., 3-6 kHz); narrow-band masking measures and difference limens indicated impaired frequency processing and varied between subjects. Intensity discrimination fell within previously published ranges. [Work supported by the National Institutes of Health.]

\section{$4: 20$}

WW10. Frequency discrimination and signal detection in band-reject noise. David S. Emmerich, William S. Brown, and Debbie A. Fantini (Department of Psychology, State University of New York at Stony Brook, Stony Brook, NY 11794)

It is known that sinusoidal stimulation creates a broad pattern of activity in the inner ear. In an attempt to assess the importance of activity remote from the peak of this pattern to subjects given a frequency discrimination task, the tones to be discriminated were present in a "notch" in a background of band-reject noise. The width of the notch was varied in different blocks of trials. For purposes of comparison, the subjects were also given the task of detecting tones presented in the notches of various widths in band-reject noise. The results indicate that when subjects are given frequency-discrimination and signal-detection tasks (adjusted to be of approximately equal difficulty in a white noise background), information is integrated over a wider range in the frequency-discrimination task. A further experiment was conducted in which a noise floor was present in addition to band-reject noise. Again, information from regions remote from the peak of the pattern of activity was found to be more important for frequency discrimination than for detection.

\section{$4 \cdot 35$}

WW11. A possible physiological correlate of frequency discrimination. John H. Mills and Richard A. Schmiedt (Department of Otolaryngology, Medical University of South Carolina, Charleston, SC 29425)

The ability of an observer to detect a change in the frequency of a signal is called frequency discrimination $(\Delta f)$. Frequency discrimination is dependent upon both the signal level and signal frequency. Here, the hypothesis is that frequency discrimination at $1.0 \mathrm{kHz}$ and above is based upon a principle of edge detection of excitation patterns present in the auditory nerve. For lack of more complete data, the high-frequency slopes of tuning curves of primary fibers are used as the criterion measures of the edge detector. There is excellent qualitative agreement between psychophysical data, which describe $\Delta f$ as a function of signal level and signal frequency, and physiological data, which describe the high-frequency slope of a tuning curve as a function of signal level and characteristic frequency. A quantitative test requires additional physiological and psychophysical data. [Supported in part by an NINCDS contract and a grant from NIEHS.]

\section{4:50}

WW12. Frequency discrimination for single channel electrocutaneous signals. Dale Bull, Rebecca E. Eilers, D. K. Oller, and Baiju D. Mandalia (Mailman Center for Child Development, University of Miami, P.O. Box 016820, Miami, FL 33101)

Frequency discrimination of electrocutaneous signals was investigated for several different base frequencies at signal durations of 50,100, and $250 \mathrm{~ms}$. Bipolar pulses (height $=10 \mathrm{~mA}$, width $=13 \mu \mathrm{s}$ ) were presented on a single electrode placed $1 \mathrm{~cm}$ above and $8 \mathrm{~cm}$ to either the left or right 
of the navel. Three practiced subjects judged pairs of stimuli (ISI $=300$ $\mathrm{ms}$ ) in a same-different task. For signal frequencies below $160 \mathrm{~Hz}$, psychometric functions were similar for all subjects and for all signal durations. In this range of frequencies, $\Delta f / f$ was constant at approximately 0.3 . By contrast, for stimuli greater than $200 \mathrm{~Hz}$, the Weber fraction was found to increase both as a function of frequency and signal duration. The results will be discussed in terms of possible intensity coding schemes for electrocutaneous vocoder designs.

THURSDAY AFTERNOON, 11 NOVEMBER 1982

PINELLAS ROOM, 4:10 TO 5:30 P.M.

\title{
Session XX. Architectural Acoustics VI and Noise VII: Noise Control and Energy Conservation
}

\author{
David Fagen, Chairman
}

Fagen \& Associates, Inc., 695 Central Avenue, St. Petersburg, Florida 33701

\author{
Chairman's Introduction-4:10
}

\section{Contributed Papers}

\section{$4: 15$}

XX1. Portable programmable calculator control of acoustic analyzer. Michael Brack (Towne, Richards, and Chaudiere, Inc., 105 N.E. 56th Street, Seattle, WA 98105)

The recent introduction of the Hewlett-Packard Interface Loop (HPIL) has provided the means for interfacing the Ivie IE-30A 1/3-octave, real-time analyzer to the $\mathrm{HP}-41 \mathrm{C}$ programmable calculator resulting in a small portable system for detailed spectrum analysis in the field. A battery-powered interface is described which, in conjunction with the HP-IL converter, allows the HP-4IC to read in the 1/3-octave or octave band spectrum to its memory with $0.2-\mathrm{dB}$ resolution over a $50-\mathrm{dB}$ dynamic range. The weighted level from the SLM portion can also be read into memory with about $0.5-\mathrm{dB}$ resolution. Some of the programs developed for this system are described. These include a program to average spectra for use in determining the space-average SPL in a room from a moving microphone, which can then be printed out, stored to memory, magnetic card, or cassette for use in noise reduction, equalization or other calculations. Machine language programming of the $\mathrm{HP}-41 \mathrm{C}$ will also be discussed for use in speeding up data manipulations by a factor of 10 to 50 times over that of normal user code.

\section{4:30}

XX2. Traffic noise and the open window. Anita B. Lawrence and Marion A. Burgess (Graduate School of the Built Environment, University of N.S.W., Kensington, N.S.W. 2033, Australia)

Natural ventilation is often the only means of providing fresh air in domestic buildings and it may become the preferred method for other buildings as well, in order to conserve energy. The simplest way of achieving natural ventilation is to open a window or door, but the sound attenuation of the building envelope may be severely reduced. The effect of an opening on facade attenuation may be predicted, if the sound transmission loss values of the facade elements are known. The accuracy of such predictions has been investigated by measuring the traffic noise attenuation of the facades of an experimental building. Various percentages of open window area were examined, and the effect of staggering the opening sashes in a spaced double window was measured. [Work supported by A.R.G.C. and S.P.C.C.]

\section{$4: 45$}

XX3. Boundary absorption for noise control and thermal insulation. David K. Holger (Department of Engineering Science and Mechanics, 214 ERI Building, Iowa State University, Ames, IA 50011)
Interior industrial sound level reductions of from 3-6 dBA can often be achieved by adding acoustically absorptive materials to the space involved. When absorption is added at the boundaries, typically the ceiling and/or walls are treated, the thermal insulation provided by the boundaries may also be enhanced. Design procedures for situations involving combined sound absorption and thermal insulation are discussed. A method for estimating sound level reduction, thermal energy conservation, and economic benefits of a combined approach is presented. The method is discussed in terms of a detailed case study.

5:00

XX4. Interior noise levels in passive solar residences. David K. Holger (Department of Engineering Science and Mechanics), and Mary S. Pickett (Departments of Family Environment, Art and Design, Iowa State University, Ames, IA 50011)

Typical single family dwellings with passive solar heating systems involve large open plan areas. Multilevel designs often incorporate open plans within and between levels. Current design practice for passive solar residences commonly utilizes hard interior surface treatments and furnishings-primarily wood, metal, glass, or masonry. The combination of open plan design and acoustically hard surfaces can result in unacceptable interior noise levels. The results of a detailed noise survey conducted in a typical passive solar home are presented. The data reveal that household appliances, HVAC equipment, and home entertainment equipment can all cause excessive interior noise levels. Sound levels in excess of $80 \mathrm{dBA}$ and NC values greater than 60 were observed in living areas of the home. These results indicate that boundary absorption is as important in open plan residential design as it is known to be in open plan commercial and institutional design. [Work supported by the Agriculture and Home Economics Experiment Station and the Engineering Research Institute.]

\section{$5: 15$}

XX5. A case history of noise control for a laboratory with fume hoods. Ronald L. McKay (Bolt Beranek and Newman Inc., P.O. Box 633, Canoga Park, CA 91305)

Centrifugal fans of fiberglass construction and with radial blades were employed to exhaust fume hoods in a medical research facility. The result was high sound levels with annoying blade-passage tonal peaks both indoors and outdoors. Initial sound levels will be presented, as well as reduced levels after the installation of duct silencers. Level distributions within rooms will be shown. Comparisons will be made between actual measured insertion loss of silencers and published insertion loss. Silencer acoustical performance with respect to location in the fan-duct system will be discussed. [Work supported by Daniel, Mann, Johnson, and Mendenhall.] 


\title{
Session YY. Psychological Acoustics VI: Pitch, Complex Waveforms, Localization, and Loudness
}

\author{
William A. Yost, Chairman \\ Parmly Hearing Institute, Loyola University of Chicago, 6525 N. Sheridan Road, Chicago, Illinois 60626
}

Chairman's Introduction-8:30

\section{Contributed Papers}

$8: 35$

YY1. The search for a biological survival value of music. Juan G. Roederer (Geophysical Institute, University of Alaska, Fairbanks, AK 99701)

A most basic issue in the study of music perception is the question of why humans are motivated to pay attention to, or create, musical messages, and why they respond emotionally to them, when such messages seem to convey no real-time relevant biological information as do speech, animal utterances, and environmental sounds. Expanding on previous work [J. G. Roederer, Physics and Psychophysics of Music (Springer, New York, 1979), 2nd ed.], four possible concurrent factors will be examined: (1) the inborn motivation to train language-handling networks of the brain in the processing of simple, organized sound patterns as a prelude to the acquisition of language; (2) the specialization of the cerebral hemispheres in information processing, in which short-term sequencing as required in speech communication and thinking is separated from holistic pattern recognition as required in music; (3) the fact that in humans limbic system functions can be activated by internally evoked images in complete detachment from the current state of environment and organism; (4) the value of music and rhythm as a means to congregate, behaviorally equalize, and even control masses of people.

8:50

YY2. Time separation pitch discrimination of signals consisting of three pulses with various periodicities. Marion G. Ceruti, Robert W. Floyd, and Douglas W. Martin (Naval Ocean Systems Center, Hawaii Laboratory, P.O. Box 997, Kailua, HI 96734)

The ability of human listeners to discriminate between equal and unequal pulse separations in signals containing pulse triplets was measured in psychoacoustic experiments. Stimuli were composed of three identical broadband pulses, each of $1.0-\mathrm{ms}$ duration centered at $2.4 \mathrm{kHz}$. The time occurrence of the third pulse was held constant relative to the first (a delay of $4 \mathrm{~ms}$ ) while the delay between the first and second pulses was varied between 1.75 and $2.25 \mathrm{~ms}$. In the standard signal, the delays between the first and second pulses and the second and third pulses were both $2.0 \mathrm{~ms}$, while in test signals these delays were made unequal by changing the temporal position of the second pulse. Subjects responded either "test" of "standard" upon presentation of the stimuli. The results show that signals with unequal pulse delays can be discriminated from a signal with equal delays when at least a $0.14-\mathrm{ms}$ difference exists between the time interval between first and second pulse of the standard and the corresponding interval in a test signal. Some subjects reported perceiving two Time Separation Pitches (TSP) in most test signals, in contrast to a higher, single TSP in the standard.

\section{9:05}

YY3. Multiple pitch induced by frequency modulation of partials of complex tones. B. Espinoza-Varas and A. S. Bregman (Psychology Department, McGill University, Montreal, Canada, H3A 1B1)

The stimuli were complex tones consisting of the first 12 harmonics of $100 \mathrm{~Hz}$, with duration of $1.5 \mathrm{~s}$. The even-numbered harmonics of the complex had a linearly falling or linearly rising spectral envelope and were frequency modulated by either a sinusoid with $90^{\circ}$ or $270^{\circ}$ phase or by a linear ramp. Frequency and depth of modulation were $0.66 \mathrm{~Hz}$ ( 1.0 cycle/ $1.5 \mathrm{~s}$ ) and $6 \%$, respectively. The odd-numbered harmonics had a flat spectral envelope and were frequency modulated by a $0^{\circ}$ phase sinusoid with frequencies of $0.3,0.66$, or $2.0 \mathrm{~Hz}$, and depths of 3,6 , or $10 \%$. In agreement with McAdams [J. Acoust. Soc. Am. Suppl. 1 68, S109 (1980)], we observed that when a different modulation function is impressed upon each subset of harmonics, the 12-component complex is perceived as having two pitch values, which seem to correspond to those of the individual subsets of harmonics when presented in isolation. An absolute identification experiment was conducted in which listeners were asked to identify the spectral envelope (timbre) and pattern of frequency modulation of the even-numbered harmonics of the 12-component complex. Accuracy of identification ranged from $40 \%-75 \%$ over the various stimulus conditions. In a second experiment, listeners were asked to judge the strength of the periodicity pitch of two-tone complexes $(2500$ and $3000 \mathrm{~Hz}$, or 500 and $600 \mathrm{~Hz}$ ) in which only the higher partial was frequency modulated by a linear ramp with frequency of 1.0 cycle per signal duration. Preliminary results suggest that the periodicity pitch of the two-tone complex becomes weaker as modulation depth increases. The relation between the perceptual data and the time and frequency domain characteristics of the signals will be discussed.

YY4. Pitch of components of complex tones.Robert W. Peters, Brian C. J. Moore, and Brian R. Glasberg(Department of Experimental Psychology, University of Cambridge, Downing Street, Cambridge CB2 3EB, England)

A current question in pitch theory is whether or not the low pitch of a complex sound is dependent on and derived from individual pitches of lower harmonics. If this is the case, pitch shifts due to masking, adaptation, and diplacusis should be similar for both types of pitch. Findings on this issue are equivocal. Some studies found a closer correspondence than others that the two pitches were affected differently. A basic problem in these studies was that pitch-shift effects for the harmonics were estimated by measuring effects on the pitches of pure tones that correpond to frequencies of the harmonics. In this study pitch matches were obtained to harmonics in complex tones and to the same harmonics in isolation. The results indicated that mean pitch values for harmonics in context did not differ markedly from their pure tone pitches. This is contrary to the findings of Terhardt (Seventh ICA, 1971, paper 20 S 13), and it indicates that the pitches of the lower harmonics in complex tones are not shifted by the presence of adjacent harmonics. Given that our subjects could make consistent and accurate matches to harmonics in context, this method is recommended for further studies of the relationship between component and complex tone pitches.

\section{9:35}

YY5. Comparison of microstructures in absolute threshold and in the pitch-intensity relation - a search for a place theory of pitch perception. W. M. Hartmann and M. A. Klein (Physics Department, Michigan State University, E. Lansing, MI 48824)

We have made fine-grained measurements of absolute threshold and of the pitch-intensity relation for sine tones as functions of frequency. For 
some subjects the measured microstructures have small enough error and are sufficiently stable in time that one can hope to draw conclusions from the data. For some ears, in some frequency ranges, there is little threshold microstructure. In these cases the pitch-intensity relation tends to show a frequency-independent shift. More commonly, however, there is considerable threshold microstructure, as much as $10 \mathrm{~dB}$ over one semitone. In those cases there is inevitably appreciable microstructure in the pitchintensity relation; the pitch shift may change sign six or seven times per octave. We have attempted to exploit the microstructures to test models of pitch perception for sine tones. We made standard assumptions about neural driven firing rate. We also supposed that absolute threshold indicates neural sensitivity. We found that our microstructure data are inconsistent with models in which pitch is derived from either the peak locus or the centroid of an average neural firing rate pattern. This conclusion is in agreement with a previous study of pitch shifts caused by a leading tone. [W. M. Hartmann, J. Acoust. Soc. Am. Suppl. 1 68, S109 (1980)]. [Work supported by the NSF and by the NIH.]

9:50

YY6. Effects of monaural stimulation by a low-frequency pure tone on binaural tinnitus. I. M. Young and L. D. Lowry (Department of Otolaryngology, Thomas Jefferson University, Philadelphia, PA 19107)

For a subject with binaural tinnitus pitch $10000 \mathrm{~Hz}$, loudness $50 \mathrm{~dB}$ SPL, left ear was stimulated by a continuous pure tone $250 \mathrm{~Hz}, 125 \mathrm{~dB}$ SPL, $30 \mathrm{~min}$. Immediately following cessation of stimulation, tinnitus completely disappeared from stimulated ear. Twenty-three min after cessation of stimulation, tinnitus was heard as a pitch $12445 \mathrm{~Hz}$. One h after stimulation, tinnitus pitch changed to $12131 \mathrm{~Hz} ; 2 \mathrm{~h}$ later, $11000 \mathrm{~Hz} ; 31 \mathrm{~h}$ later, $10953 \mathrm{~Hz} ; 5 \mathrm{~h}$ later returned to original pitch $10000 \mathrm{~Hz}$ and stayed on to date. For a period of $23 \mathrm{~h}$ after stimulation, however, stimulated ear continuously heard multiple frequency tones approximately $200-800 \mathrm{~Hz}$. In nonstimulated ear, immediately after cessation of stimulation, tinnitus was heard as a pitch $5000 \mathrm{~Hz}$ and gradually increased in pitch to $7500 \mathrm{~Hz}$ for $31 \mathrm{~h}$. Thereafter, tinnitus pitch has been fluctuating between 9000 $10000 \mathrm{~Hz}$. Tinnitus never returned to previous pitch $10000 \mathrm{~Hz}$ until 16 days after stimulation. Apparent differences in recovery pattern and process between two ears were compared with a previous experiment of 500 $\mathrm{Hz}$ monaural stimulation.

\section{0:05}

YY7. Learning a "tonal" vocabulary. M. R. Leek" and C. S. Watson (Boys Town Institute for Communication Disorders in Children, 555 North 30th Street, Omaha, NE 68131)

At the last meeting of this Society, we described the time course of learning to detect individual tonal components of a sequential pattern [Leek et al., J. Acoust. Soc. Am. Suppl. 1 71, S37 (1982)]. In this study, the investigation of auditory perceptual learning was extended to an identification task. Seven listeners were first trained to identify four 3-tone minipatterns or "syllables," using numbered response keys. All possible combinations of these syllables (without repetition) provided twenty-four 4-syllable "words," which the listeners were subsequently asked to identify by naming the order of the syllables. After several weeks of daily practice, most listeners learned to correctly identify the words at $80 \%$ correct or better. Although rates of learning varied among listeners, the learning curves displayed similar characteristics. As identification became more accurate, response latencies dropped by $60 \%-70 \%$, supporting subjective reports of an increasingly automatic and unitary perception of the words. An analysis of perceptual confusions and how they were resolved over time shows that some words were learned as whole units. However, many apparently were learned as concatenations of 1-, 2-, or 3-syllable units, perhaps reflecting the creation of a primitive phonological system by the listener. [Work supported by NIH.] ${ }^{\text {a) }}$ Current address: Department of Speech and Hearing Science, Arizona State University, Tempe, AZ 85281 .

10:20

YY8, Listeners' identification of environmental sounds. Norman J. Lass, Sandra K. Eastham, William C. Parrish, Kathleen A. Scherbick, and
Dawn M. Ralph (Department of Speech Pathology and Audiology, West Virginia University, Morgantown, WV 26506)

The purpose of this investigation was to determine if listeners can accurately identify various environmental sounds. A total of $\mathbf{4 0}$ sounds were recorded: (a) 20 animal sounds from four cows, cats, dogs, pigs, and sheep; (b) 12 inanimate sounds from four typewriters, car horns, and telephones; (c) four musical sounds from a flute, piano, guitar, and drum; and (d) four human sounds $\left(/ \mathrm{p}_{\mathrm{N}}\right)$ from two female and two male speakers. A master tape containing the randomly arranged recordings was prepared and presented to 30 judges who were asked to identify the source of each sound and the confidence of their judgments on a seven-point rating scale. Results indicate that listeners can accurately identify environmental sounds. However, their accuracy was not equal within each sound class as well as between all four classes of sounds investigated: human sounds yielded the highest accuracy, followed by musical and inanimate sounds, and animal sounds were least accurately identified. Moreover, listeners' judgmental confidence was similar to that of their accuracy. Implications of these findings and suggestions for future research are discussed.

\section{0:35}

YY9. Interaction of interaural time and intensity. William A. Yost (Parmly Hearing Institute, Loyola University of Chicago, $6525 \mathrm{~N}$. Sheridan Road, Chicago, IL 60626)

The thresholds for interaural temporal and intensive differences were determined as a function of a variety of stimulus conditions. The thresholds were determined in a two-alternative, forced-choice task when the stimuli were presented with a constant interaural temporal or intensive difference. That is, the thresholds were measured in conditions in which the lateral image is moved of midline with the introduction of an interaural temporal or intensive difference. A random change in overall level prevented the listener from using monaural intensive or binaural loudness differences as cues for discrimination. The thresholds were measured as a function of signal frequency $(200-1200 \mathrm{~Hz})$ for 250 -ms tones presented at $60 \mathrm{~dB}$ SPL. Both interaural temporal and intensive thresholds increased as the value of the constant interaural difference increased (images moved away from midline). The interaural temporal difference threshold changed from approximately $3^{\circ}$ to approximately $18^{\circ}$ as the constant interaural intensive difference increased from 0 to $15 \mathrm{~dB}$. The interaural intensive threshold changed from approximately 1 to approximately $2.1 \mathrm{~dB}$ as the constant interaural temporal difference changed from $0^{\circ}$ to $180^{\circ}$. The effects of frequency were small but significant. The results will be discussed in terms of the relationship between lateralization and localization of pure tones. [Work supported by The National Science Foundation.]

\section{0:50}

YY10. Localization of sound in rooms. W. M. Hartmann (Institut de Recherche et Coordination Acoustique/Musique, 31 rue Saint Merri, 75004 Paris, France)

We have studied the ability of human listeners to locate the origin of a sound in a room in a series of source azimuth identification experiments. All experiments were done in a small rectangular concert hall with variable geometry and acoustical properties. Subjects localized a 50-ms, 500$\mathrm{Hz}$ sine pulse with an rms error of $3.3^{\circ}\left( \pm 0.6^{\circ}\right)$ regardless of room reverberation time. Lowering the ceiling from 11.5 to $3.5 \mathrm{~m}$ decreased the error to $2.8^{\circ}\left( \pm 0.6^{\circ}\right)$. Subjects localized broadband noise without attack transients with an rms error of $2.3^{\circ}\left( \pm 0.6^{\circ}\right)$ if the reverberation time was $1 \mathrm{~s}$. The error increased to $3.2^{\circ}\left( \pm 0.7^{\circ}\right)$ if the reverberation time was $5 \mathrm{~s}$. For complex tones without attack transients the localization error continuously increased as the intensity of spectral components decreased. Performance was nearly random for a $500-\mathrm{Hz}$ sine tone, but was significantly better than random for a $5000-\mathrm{Hz}$ sine tone. Our azimuth identification experiments revealed significant biases, as much as $2^{\circ}$; such biases are, of course, invisible in minimum audible angle experiments.

\section{1:05}

YY11. Binaural horizontal plane localization: Influence of monaural spectral cues. Alan D. Musicant and Robert A. Butler (Departments of 
Behavioral Sciences and Surgery, University of Chicago, Chicago, IL 60637)

A recent article [Butler and Flannery, Percept. Psychophys. 28, 449457 (1980)] reported the existence of monaural spatial referent maps (SRMs). These orderly maps related the perceived horizontal plane locus of narrow bands of noise to center frequency, which ranged from 4-14 kHz. Perceived location was governed primarily by spectral composition and not actual source location. Similar SRMs under binaural listening conditions were obtained in the current experiments. Stimuli were presented from a loudspeaker at $270^{\circ}$ azimuth. Subjects could report the perceived origin as any of 13 positions, each $15^{\circ}$ apart and extending from $360^{\circ}$ to $180^{\circ}$. Results were consistent with observations regarding the region of the horizontal plane most sensitive to spectral cues. [Musicant and Butler, J. Acoust. Soc. Am. Suppl. 1 71, $\$ 87$ (1982)]. Localization tests utilizing specific features of the SRMs decreased binaural localization accuracy. A 4-kHz high-pass noise with a notch at a "map-connecting" region was the stimulus. Stimuli were presented from all 13 loudspeakers in the horizontal plane. Performance decrements were greatest within the $315^{\circ}-225^{\circ}$ arc. The present results strengthen our argument that monaural spectrol cues are an important cue to binaural horizontal plane localization.

\section{1:20}

YY12. Growth rate of perceived magnitude as a function of tone-to-noise ratio. Rhona Hellman (Communication Sciences Laboratory, Boston University, Boston, MA 02215)

The growth of overall perceived magnitude (loudness and annoyance) of single tones superimposed on broadband-flat, low-pass, and high-pass noise spectra was measured by absolute magnitude estimation (AME). Results disclose that, in contrast to noiseness, loudness and annoyance growth are dependent on the relationship between the frequency of the added tone and the spectral shape of the noise. Tones centered in noise produce nonmonotonic loudness and annoyance growth functions; those added to the noise skirt produce power functions. Detailed analysis reveals that the measured exponents are invariant across tone-to-noise ratio when the tones are positioned within the noise spectrum, but not when they are added to the skirt. A high-frequency tone combined with lowpass noise increases the growth rate of perceived magnitude as tone-tonoise ratio increases. Conversely, the addition of a low-frequency tone to high-pass noise decreases the growth rate of perceived magnitude. The obtained results are qualitatively compatible with excitation patterns known to be evoked by auditory stimuli at high intensities. [Work supported by NASA Langley Research Center.]

\section{1:35}

YY13. Measurements of equal loudness and reaction times. Søren Buus, Hilda Greenbaum, and Bertram Scharf (Auditory Perception Laboratory, 413MU, Northeastern University, Boston, MA 02115)

Kohfeld et al. [Percept. Psychophys. 29, 535-549 (1981)] suggested that reaction times may not be the same for equally loud stimuli. However, their equal-loudness contours showed unusually large level differences between the $1000-\mathrm{Hz}$ standard and the $500-$ and $5000-\mathrm{Hz}$ comparisons. To investigate the effect of the loudness-matching procedure used by Kohfeld et al., four listeners made equal-loudness judgments using three different methods: (1) a method of limits with the standard and comparison alternating between ears, similar to Kohfeld et ol.'s procedure, (2) a method using three interweaved adaptive procedures with a $2 \mathrm{I}, 2 \mathrm{AFC}$ paradigm and monotic, random-order presentation of standard and comparison, and (3) the method of adjustment with monotic, alternating presentation of standard and comparison. Results from the first method resembled those of Kohfeld et al: : at 20, 40, and 60 phons, the 500- and $5000-\mathrm{Hz}$ comparisons were set about $8 \mathrm{~dB}$ above the $1000-\mathrm{Hz}$ standard. Results from the second and third methods agreed with each other, but were different from those obtained with the first method: at all three loudness levels, the comparisons were set only a few $\mathrm{dB}$ above the standard. Preliminary measurements indicate that reaction times to equally loud tones, as determined with the second and third methods, were approximately equal. [Work supported by NIH grant RR07143.]

\section{1:50}

YY14. Loudness functions of short-duration tone glides from nonmetric scaling. Kenneth L. Pilgreen (Department of Neurology, Medical College of Virginia, Richmond, VA 23298) and John K. Cullen, Jr. (Kresge Hearing Research Laboratory of the South, Department of Otorlaryngology, LSU Medical Center, New Orleans, LA 70119)

Individual loudness functions of short-duration $(20 \mathrm{~ms})$ tone glides and pure tones were obtained for three listeners. Upsweep and downsweep tone glides varied linarly between endpoint frequencies of 500 and 2500 $\mathrm{Hz}$; binary judgments were made by subjects at ten intensities between 30 and $90 \mathrm{~dB}$ SPL. Subjects judged loudness differences between tone pairs, and loudness projections were derived using a multidimensional unfolding program developed by Schneider [J. Acoust. Soc. Am. 69, 1208-1209 (1981)]. Loudness differences between the tone glides and pure tones occurred over most of the range of intensities; some differences occurred between upsweep and downsweep tone glides at the highest levels tested. These results indicate that for short-duration signals with rapid changes in frequency, loudness growth is a steeper function that for pure tones of similar duration.

\section{2:05}

YY15. Aided and unaided localization on subjects with different degrees and types of losses. R. Wayne Gatehouse and C. L. Patee (Department of Psychology, University of Guelph, Guelph, Ontario, NIG 2W1 Canada)

Binaurally deaf persons categorized by (1) three degrees of ac measured losses (26-35 dB, minimal; 36-45 dB, mild; 46-55 dB, moderate) and (2) clinical categories ( $\mathrm{SN}$, mixed $(\mathbf{M})$, or conductive $\mathrm{C}$ ) were tested on accuracy in "simultaneous" localization (i.e., specification of signal position in both azimuth and elevation). For some subjects this was done with and without their hearing aids. Four signals (WN; 1.0 - and $2.5-\mathrm{kHz}$ tones; and speech) equated for $72 \mathrm{~dB}$ SPL at head position were localized. Three 24-trial randomized blocks of eight degrees azimuths $\left(0^{\circ}, 45^{\circ} \ldots 315^{\circ}\right) \times 3$ elevations $\left(0^{\circ}, \pm 30^{\circ}\right)$ were presented for each signal under all conditions. The orders of signal presentations and blocks were randomly varied. The results like those reported by Tonning (1975) indicate that degree of loss as measured by pure tone audiograms may be useful predictors of localization performance-errors do increase (especially in azimuth) as the loss severity does. However, other factors (age; symmetry/not of audiogram) are also important. Additionally, loss type (SN, M, or C) seems to give some basis for prediction of localization ability. Finally, as in Gatehouse (1981) head-borne monaural aids do appear to produce inferior localization, especially for speech and WN signals. The results will be considered in light of Tonning's long series of articles (1970-75) and in terms of possible diagnostic utility of including localization tests. [Work supported by MRC Canada.] 


\title{
Session ZZ. Underwater Acoustics VIII: Seismic Acoustics
}

\author{
James M. McKisic, Chairman \\ Office of Naval Research, 800 North Quincy Street, Arlington, Virginia 22217 \\ Chairman's Introduction-8:30 \\ Invited Papers.
}

8:35

ZZ1. Synthetic seismograms. C. H. Chapman (Department of Physics, University of Toronto, Toronto, Ontario M5S 1A7, Canada) and J. A. Orcutt (Geological Research Division, Scripps Institution of Oceanography, University of California, San Diego, La Jolla, CA 92093)

The interpretation of seismic data, in particular oceanic refraction data, has improved considerably, in recent years using synthetic seismograms. Several different methods have been developed for computing synthetic seismograms and within the limitations of the various approximations, shown to give equivalent results. The computation of theoretical seismograms can be divided into two parts: the evaluation of the response in a transform domain (normally frequency and horizontal slowness), and the evaluation of the inverse transforms (Fourier, Fourier-Bessel, or Legendre transforms). Developments of the Haskell matrix method are widely used to evaluate the transformed response. Problems with numerical stability have been solved, and the method has been extended to use asymptotic solutions in inhomogeneous layers. Often the complete response is expanded, at least at some interfaces, into "rays" and only a limited set of rays investigated. The ray expansion often simplifies the evaluation of the inverse transforms and takes advantage of separation in the time domain. The inverse transform methods fall into two catagories: spectral and slowness methods. In spectral methods, the inverse wavenumber transform is evaluated first and the intermediate result is the spectral response for a point source. In slowness methods, the inverse frequency transform is evaluated first and the intermediate result is the impulse "plane wave" response. For a general response function, neither method is numerically preferable but the intermediate result in the slowness method is convenient as it corresponds to slant stacking the data. With the ray expansion, the slowness method is often preferable as the inverse transforms can be evaluated exactly - the generalized ray method and the WKBJ seismogram. The last method has recently been extended to laterally inhomogeneous media.

8:55

2Z2. Experimental estimates of the energy partitioning in long-range, low-frequency seismic-acoustic propagation. A. B. Baggeroer (M.I.T., Cambridge, MA 92139)

Several recent experiments have used arrays to resolve the multipath in long range, low-frequency seismic/ acoustic propagation. These experiments have been conducted in several geologic environments including, (i) the thickly sedimented Canada Basin (CANBRAX); (ii) the abyssal plains of the Eastern Arctic (FRAM II and FRAM IV); and (iii) the young crust of the East Pacific (ROSE). In characterizing the seismic/acoustic propagation the array data are used to estimate slowness-travel time spectra. These are first transformed to a " $\tau$ - $p$ " spectra and migrated to a velocity-depth spectra to estimate a crustal model. The slowness-travel time spectra are next used to measure the energy partitioning among the multipaths, or equivalently their scattering function, and their attenuation, or " $Q$ " factors. Finally, they are used to estimate the boundary effects of the seabed upon the model energy to complete the analysis of the seismic/acoustic propagation. Each of the geologic environments generates very diverse multipath structure. The effects of bottom roughness, sediment thickness, and velocity gradients upon the crustal models, energy partitioning of the multipaths and the model energy will be illustrated using experimental results from the array data.

\section{9:15}

Z73. Shallow water infrasonics: Propagation and noise studies with ocean bottom seismometers. D. Rauch and B. Schmalfeldt (SACLANT ASW Research Centre, 19026 La Spezia, Italy)

Sound propagation studies near or below the hydroacoustic cutoff frequency of shallow water ducts have been strongly neglected in the past. The growing interest in bottom-interacting phenomena stimulated the investigation of pertinent propagation mechanisms to identify low-frequency sources or to probe the upper sea floor in-situ. For this purpose three-component ocean bottom seismometers each with a radio link and an external hydrophone have been developed and deployed during several sea trials off the Italian coast near La Spezia. Numerous measurements from srnall underwater explosions and from the ambient background or passing ships were analyzed with various software packages. The results to be discussed in this paper are 
confined to the infrasonic regime from 1 to $18 \mathrm{~Hz}$. Shot-data are illustrated by typical seismogram sections, particle hodographs, dispersion contours for amplitude or phase, and attenuation curves, while noise data are presented in the form of amplitude or cross spectra. It can be clearly demonstrated that the infrasonic energy is mainly transmitted via seismic interface waves of the Stoneley-or Scholte-type propagation along the sea floor.

9:35

ZZ4. Wave attenuation. Paul G. Richards (Lamont-Doherty Geological Observatory and Department of Geological Sciences of Columbia University, Palisades, NY 10964)

It is commonly observed that propagating waves lose amplitude sooner than might be expected for elastic media with smoothly varying properties. The explanation is in part that real materials are not elastic, and in part that real materials are not smoothly varying but have fine scale spatial fluctuations. We thus speak of anelastic attenuation, and attenuation due to scattering. Anelastic attenuation can be understood in a framework that allows for complex moduli relating stress and strain. A brief review will be given, of successful procedures for accommodating anelasticity in the computation of waveforms. The main part of this paper concerns attenuation of transmitted waves when forward scattering is dominant. This is the case for an elastic medium with a heterogeneous velocity structure when the typical fluctuation is spatially confined within less than a wavelength and the wave propagates over a sufficiently long path. The medium is found to have low-pass characteristics. The removal of high frequencies can often be summarized by a frequency-independent apparent $Q$. It is important in seismic prospecting to be able to determine whether observed amplitude decay is due to the presence of anelasticity and true dissipation, or to the presence of velocity fluctuations and resultant scattering. Numerical experiments indicate that when scattering dominates over anelasticity: (1) The coda of a transmitted wave contains relatively higher frequencies than the initial phase; (2) the attenuation deduced from the power spectrum of the transmitted wave is greater than that deduced from the phase spectrum; (3) compressional and shear wave apparent $Q$ 's are approximately equal; and (3) estimates of apparent $Q$ made from reflected coda vary with frequency, while estimates made from the transmitted waves do not.

ZZ5. Inversion for sediment structure using multichannel data. Robert A. Phinney (Department of Geological and Geophysical Sciences, Princeton University, Princeton, NJ 08544)

The problem of inferring a velocity or impedance structure for a layered sediment has traditionally been posed in terms of a restricted data set, such as a normal incidence reflection seismogram or a refraction travel time curve. Data acquisition systems used for petroleum exploration record 96 or more channels in a linear array, and thus obtain a virtually unaliased representation of the wavefield. The data contains reflections from all angles of incidence, including postcritical angles, all normal modes, leaky modes, and head waves. A key tool in dealing with this kind of data is the Radon transform, which transforms the data set into a plane-wave representation. A raw data set $u(x, t)$ is transformed into $v(p, \tau)$, where $\rho$, which parameterizes the plane waves, is the $x$ component of slowness, and $\tau$ is a reduced (intercept) time. The ensemble of plane-wave seismograms is the basis for inversion of the data to obtain a one-dimensional velocity/impedance model. Modeling the lossy elastic transient plane-wave response is easily done by one of the modifications of the Thomson-Haskell matrix method. The algorithm can be vectorized to run very rapidly on a machine with an array processor. The object of the inversion is to obtain a model whose plane-wave decomposition agrees exactly with that of the observed wavefield. In the absence of noise, one plane-wave seismogram suffices to determine the model. An ensemble for a range of wave parameters overdetermines the model. In the presence of noise, the problem has the character of an ill-posed, overdetermined least squares problem. In order to carry through the solution by means of least squares, it is necessary to go through a construction procedure to make the trial model linearly close to the correct one in some parameter subspace. Several construction procedures proceed by stripping from the top down, although none have been reduced to a fully automatic alogrithm, and require some human intervention in the iterations.

ZZ6. Ocean bottom seismic instrumentation. Frederick K. Duennebier (Hawaii Institute of Geophysics, 2525 Correa Road, Honolulu, HI 96822)

During the past several years the quality of seismic data obtained in the deep oceans has improved markedly due to the development of better instrument packages and recording systems. These improvements take advantage of improved understanding of the seismic coupling problem, the effects of ocean currents, and Stoneley waves. The coupling problem arises from the inability of an ocean bottom seismometer (OBS) to move with the ocean bottom in response to seismic waves when the bottom is soft. The transfer function between the motion of the bottom and the OBS is good at very low frequencies and poor at high frequencies, the corner depending on the softness of the bottom and the mechanical characteristics of the OBS. The buoyancy of the OBS also has a pronounced effect on the response. These coupling effects are not observed on hydrophones. Several solutions to the above problems are being proposed and tested. First, since many of the problems with collecting high quality seismic data on the ocean floor are related to the ocean bottom interface, one solution is to remove the sensors from the interface and place them in drill holes under the ocean floor. This operation is considerably more expensive than emplacing OBS's but preliminary results suggest that the increase in data quality is significant. Solutions to the coupling problem include sensor burial, increasing the surface area in contact with the ocean bottom, and making sensor packages neutrally buoyant in the sediments. Current noise problems are 
greatly improved by decreasing the OBS cross section to the water. Stoneley wave generated noise is truly seismic and can be discriminated against by using multiple sensors or hydrophones that are much less sensitive to these signals.

\section{Contributed Papers}

\section{0:35}

ZZ7. Seismic survey signals in the Canadian Beaufort Sea. Charles R. Greene (Polar Research Laboratory, 123 Santa Barbara Street, Santa Barbara, CA 93101)

In conjunction with a study of the responses of bowhead whales to noises from petroleum industry activities in the Beaufort Sea, we recorded signals received from sleeve exploders at a number of ranges from 8 to 29 $\mathrm{km}$. We investigated the detailed nature of these signals, including their spectral content and waveforms, and used the results to describe sound transmission loss in the shallow $(<50 \mathrm{~m})$ waters of the southeastern Beaufort Sea. The major part of the received signal began at about $200 \mathrm{~Hz}$ and swept down to about $100 \mathrm{~Hz}$. The duration ranged from $250 \mathrm{~ms}$ at $8 \mathrm{~km}$ to over $400 \mathrm{~ms}$ at $29 \mathrm{~km}$, with reverberation extending much longer. We derived a regression model $\left(R^{2}=0.972,12\right.$ measurements) with cylindrical spreading plus an absorption loss term of $1.39 \mathrm{~dB} / \mathrm{km}$ for signal components around $150 \mathrm{~Hz}$. [Work supported by the U.S. Bureau of Land Managernent.]

\section{0:50}

ZZ8. Application of a Biot/Stoll model in acoustic simulations. Burlie A. Brunson and Eugene Molinelli (Planning Systems Inc., 7900 Westpark Drive, Suite 600, McLean, VA 22102)

A physical sediment model based on Biot Theory as modified by Stoll is used to predict the geoacoustic properties of the sea floor. The sediment properties are predicted as functions of depth and frequency for use as inputs to acoustic simulation models. The geoacoustic predictions are compared with geoacoustic descriptions derived from inversion of acoustic bottom loss data. The acoustic significance of observed differences is assessed using acoustic simulations such as bottom loss and propagation loss. The sensitivity of geoacoustic predictions to the variability of sea floor geophysical properties is discussed. [Work supported by ONR and NORDA.]

\section{1:05}

ZZ9. Deterministic geoacoustic model for a randomly layered ocean bottom. Kenneth E. Gilbert (Naval Ocean Research and Development Activity, Numerical Modeling Division, NSTL Station, MS 39529)

A deterministic wave equation for the mean acoustic field in a randomly layered medium is derived. The wave equation is used to define a "smooth" deterministic geoacoustic model which is acoustically equivalent at low frequencies to a randomly layered model. The wave equation derivation starts with fundamental relations (Newton's law and Hooke's law) and leads to deterministic expressions for effective sound speed, density, and absorption which are similar to those obtained for periodic medium. In particular, the effective sound speed of the deterministic model depends on the grazing angle, being higher for propagation parallel to the layering than for propagation perpendicular to it. When the angular dependence of the effective sound speed is included, the low-frequency ( $f<100-200 \mathrm{~Hz}$ ) reflection coefficients for the deterministic model are in good agreement with the ensemble averaged coefficients for the associated randomly layered model. Without the angular dependence, the agreement is marginal.

\section{1:20}

ZZ10. The application to real data of a technique for measuring the planewave reflection coefficient of the ocean bottom. George V. Frisk, Douglas R. Mook, James A. Doutt, Earl E. Hays (Woods Hole Oceanographic Institution, Woods Hole, MA 02543), and Alan V. Oppenheim (Research Laboratory of Electronics, Massachusetts Institute of Technology, Cambridge, MA 02139)
A technique has been described for the measurement of the planewave reflection coefficient of a horizontally stratified ocean bottom [G. V. Frisk, A. V. Oppenheim, and D. R. Martinez, J. Acoust. Soc. Am. 68, 602-612 (1980)]. It is based on the exact Hankel transform relationship between the reflection coefficient and the bottom reflected field due to a point source, and was shown to yield excellent results with synthetically generated data for simple bottom examples. We apply the method to real $220-\mathrm{Hz}$ data obtained using a near-bottom experimental geometry. The results of the Hankel transform inversion are compared with those obtained using an iteration of forward models technique. [Work supported by ONR, Johns Hopkins Applied Physics Laboratory, and NORDA.]

\section{1:35}

ZZ11. Propagation through refracting ocean sediments. Ioannis M. Besieris (Department of Electrical Engineering, Virginia Tech, Blacksburg, VA 24061), Werner Kohler (Department of Mathematics, Virginia Tech, Blacksburg, VA 24061), and Herbert Freese (Arete Associates, P.O. Box 2951, Arlington, VA 22202)

A transport theoretic analysis of propagation through highly anisotropic randomly perturbed sediment layers has previously been developed for the case of constant mean density and sound speed. [J. Acoust. Soc. Am. 12, 937-946 (1982); preliminary results reported in J. Acoust. Soc. Am. Suppl. 1 70, S1 (1981)]. The present paper extends this work to include the effects of refraction. The effect of variations in the mean density and sound speed upon the mean field and incoherent intensity is discussed. [Work supported by ONR Contract No. N00014-76-C-0056 and NAUSEA Code 63D.]

\section{1:50}

ZZ12. Acoustic propagation in the ocean with a porous bottom. Tokuo Yamamoto (Division of Ocean Engineering, School of Marine and Atmospheric Science, University of Miami, Miami, FL 33149)

Biot's theory was directly incorporated with the normal mode analysis of acoustic propagation in the ocean with a porous elastic bottom. The complex dispersion relations was obtained in a closed form for the case of a homogeneous ocean with a poro-elastic half-space. The attenuation data from the shallow water experiments in the Mediterranean Sea were compared with the theoretical calculations. The $100-\mathrm{m}$ water/sandy bottom data showed an optional transmission frequency of about $250 \mathrm{~Hz}$. The theoretical analysis using the sediment data revealed that the attenuation of the high-frequency components are mainly due to the seepage of pore water through the sand (the slow compressional wave effect) and the attenuation of the low-frequency components are owing to the energy dissipation of the shear waves by the Coulomb friction in the consolidated sand. As a result, the intermediate frequency components have minimum overall attenuations and result into the optimal transmission frequencies. The agreements between the theory and the experiments are good. [Work supported by NSF.]

\section{2:05}

ZZ13. Wake Island hydrophone seismic array. George $H$. Sutton, Paul W. Pomeroy (Rondout Associates, Inc., Box 224, Stone Ridge, NY 12484), Daniel A. Walker, and Charles S. McCreery (Hawaii Institute of Geophysics, University of Hawaii, Honolulu, HI 96822)

The hydrophone array installed near Wake Island (WHA) was reactivated as a seismic station in June 1979 by the Hawaii Institute of Geophysics. WHA consists of six bottom phones, at $5.5-\mathrm{km}$ depth, in an array 40 $\mathrm{km}$ across and an additional four pairs of phones, at large spacing, suspended at SOFAR depth. The bottom array lies in a flat area on high $Q$ lithosphere $>10^{\mathrm{A}}$ years old. All of the deep and five of the SOFAR phones 
are operational. Until September 1982 , generally data were recorded from only three phones, on a slow speed analog cassette recorder. The station is now being upgraded for digital recording of all 11 phones. Many circumpacific earthquakes and nuclear tests have been well recorded by WHA, its location being ideally suited for monitoring such events. Nearly pure oceanic paths between WHA and circumpacific sources facilitate studies of the ocean-lithosphere waveguide such as propagation and attenuation of high-frequency, teleseismic $\mathrm{Pn}$ and $\mathrm{Sn}$. For frequencies above $3 \mathrm{~Hz}$, WHA is quieter than most quiet continental stations. [Work supported by AFOSR and ONR.]

2Z14. A corner-reflector parametric transducer for acoustic imaging in marine sediment. G. L. Sackman, R. L. Eyman, P. J. LaStrange, and A. H. Seeman (Department of Electrical Engineering, Naval Postgraduate School, Monterey, CA 93940)

An experimental transducer and pulse modulator has been constructed which employs the parametric transient effect [M. B. Moffett, P. J. Westervelt, and R. T. Beyer, J. Acoust. Soc. Am. 49, 339-343 (1941)] for locating and identifying objects buried in marine sediment [L. A. Thompson and T. G. Muir, J. Acoust. Soc. Am. 55, 429 (1974)]. It was desired to produce a probing acoustic beam with the best possible combination of short pulse length and narrow beamwidth for good object resolution while simultaneously using the lowest possible frequency for penetration of the sediment. A novel corner reflector configuration for the transducer is used which is well adapted to operation at great depths. The transducer is shock excited at its $80 \mathrm{kHz}$ primary frequency, from which a $100 \mu \mathrm{s}$ parametrically demodulated baseband pulse is generated in a collimated beam of approximately $20-\mathrm{cm}$ diameter over ranges from 5 to $15 \mathrm{~m}$. [Work supported in part of Naval Undersea Warfare Engineering Station, Keyport, WA.]

\section{2:35}

ZZ15. Sound speed and attenuation in marine sediments measured at high frequencies with a bottom probe system. Y. Igarashi and R. L. Allman (Naval Ocean Systems Center, San Diego, CA 92152)

The evaluation of many high-frequency bottom-interaction models requires as inputs sound speed and attenuation in marine sediment. $A$ bottom probe system mounted on the underside of a cable-controlled underwater vehicle was used to make measurements at 15,30 , and $60 \mathrm{kHz}$, and at probe depth positions of 17,42 , and $67 \mathrm{~cm}$. Sound-speed ratios are determined in-situ to $0.1 \%$ and attenuation to $0.2 \mathrm{~dB} / \mathrm{m}$. Measurements made in a $1 \mathrm{~km}$ by $1 \mathrm{~km}$ area of Puget Sound indicated small but significant spatial distributions of acoustic properties in a nominally uniform area. Attenuation coefficients were determined using the empirical relation $K$ times frequency to the $n$th power. Exponent $n$ was approximately 0.70 . Past data in a Santa Barbara area indicated an $n$ value of 1.19. These results indicate that for different sediments, exponent $n$ is distributed. about one with significant deviations. [Work supported by Naval Sea Systems Command.]

\title{
FRIDAY MORNING, 12 NOVEMBER 1982
}

DADE AND FLORIDA KEYS ROOMS, 9:00 A.M. TO 12:05 P.M.

\author{
Session AAA. Physical Acoustics VIII: Interface Phenomena
}

\author{
Mack A. Breazeale, Chairman \\ Department of Physics and Astronomy, University of Tennessee, Knoxville, Tennessee 37916
}

Chairman's Introduction-9:00

\section{Contributed Papers}

9:05

AAA1. Propagation of acoustic surface-wave pulses. G. C. Gaunaurd, H. Überall (Naval Surface Weapons Center, R43, White Oak, Silver Spring, MD 20910), and J. V. Subrahmanyam (Department of Physics, Catholic University, Washington, DC 20064)

Surface waves on impenetrable or on elastic objects, produced by acoustic scattering or by other mechanisms, show pronounced dispersion effects. Their dispersion curves may be obtained from the resonances exhibited by their amplitude function either in the complex frequency plane ("SEM" or singularity expansion method poles) or in the complex mode number plane ("Watson poles" or "Regge poles") [such acoustic resonances have recently been observed and analyzed by Maze, Taconet, and Ripoche, Phys. Lett. A 84, 309 (1981)]. Accordingly, the propagation of surface-wave pulses, and their arrival times, are determined by their group velocity. This is demonstrated by summing the normal-mode series of the surface wave portion of scattering amplitudes on spherical and cylindrical surfaces, using a stationary-phase approach. At the stationary points, which determine the pulse arrival times, there occurs a coherent summation (constructive interference) of the normal modes, and the stationary condition is recognized to lead to the correct propagation of the pulses with group velocity. [H. Überall is also at Catholic University, Washington, DC and is additionally supported by ONR.]

\section{9:20}

AAA2. Dirac constraint treatment of surface waves. C. A. Uzes (Naval Coastal Systems Center, Panama City, FL 32407)

Some of the commonly used boundary conditions on the displacement field of an elastic body are classified as Dirac constraints. The consequent equations of motion for the surface waves of the elastic body are constructed for a number of surface geometries and their possible applications discussed. [Work supported by ONR/ASEE Summer Faculty Research Program.]

\section{9:35}

AAA3. Approximate closed form solution to the generalized interface wave speed equation. JoEllen Wilbur, Richard $O$. Claus, and John W. Gray (Department of Electrical Engineering, Virginia Polytechnic Institute and State University, Blacksburg, VA 24061)

An approximate closed form solution for the speed of generalized surface acoustic waves which are bound to a thin layer separating two infinite half-spaces is presented. The exact form of the characteristic equation is first derived by considering the usual requirements of particle dis- 
placement and stress continuity at both layer-substrate boundaries [J. P. Jones and J. S. Whittier, J. Appl. Mech. 905 (Dec. 1967)]. It is then noted that in many cases the interface wave speed is less than half the lower longitudinal wave speed and as much as $5 \%$ less than the lower shear wave speed. After expressing terms involving ratios of these speeds in the exact equation as series expansions, this has been used to neglect appropriate small terms and to simplify the resulting equation. Additional simplification yields approximate closed form expressions for all 12 complex roots. Variations in these solutions as a function of the viscoelastic properties of the boundary layer are compared with theoretical [V. Kumar and G. S. Murty, IEEE Trans. Sonics Ultrason. SU-29, 138 (1982)] and experimental results [R. O. Claus, J. Phys. (Paris) 42, C5-1159 (1981)]. [Work supported in part by NSF and NASA.]

\section{$9: 50$}

AAA4. Experimental observations on liquid/solid interface waves. Jacques R. Chamuel (The Charles Stark Draper Laboratory, Inc., 555 Technology Square, Cambridge, MA 02139)

Elastic waves propagating at the interface between a liquid and a solid present interesting complex phenomena as the relative elastic properties of the liquid and the solid are varied. It is difficult to obtain a good physical interpretation of the mathematical solutions of the Stoneley wave equation. Experimental results are presented using limestone/water, aluminum/water, Plexiglas/water, and ice/water. A barrier was placed between source and receiver to isolate the Scholte wave from the water and pseudo-Rayleigh waves. The experiments were repeated with a deep narrow cut made in the solid to stop the seismic waves. The measured Scholte (Stoneley) wave velocities agreed with Strick and Ginzbarg's calculations (1956). For the limestone/water case, the barrier stopped the Scholte wave and did not affect the pseudo-Rayleigh wave. The barrier did not stop the Scholte wave for the Plexiglas and ice cases. The deep narrow cut in the solid stopped the pseudo-Rayleigh wave for the limestone/water case, and did not affect the Scholte wave. The Plexiglas and ice Scholte waves were interrupted by the deep cut. The reported experimental findings provide insight into the physical understanding of interface wave propagation.

\section{0:05}

AAA5. General Rayleigh angle phenomenon. Laszlo Adler (Department of Welding Engineering, Ohio State University, Columbus, OH 43210), Michel de Billy (Groupe de Solide de Physique, Universite Paris 7), and Andy Norris (Department of Engineering Science, Northwestern University, Evanston, IL 60201)

Experimental and theoretical work is presented to investigate the interaction of finite ultrasonic beams with liquid-solid interfaces. The results indicate that for any angle of incidence leaky waves are generated. These leaky waves are radiated to the liquid from the solid suface at the Rayleigh angle. For a general angle of incidence the amplitude of these leaky waves are much smaller than the amplitude of the specularly reflected wave. A special case of this general phenomenon is the Rayleigh angle incidence which produces the null strip in the forward direction and low amplitude scattering in the backward direction.

\section{0:20}

AAA6. Nonspecular reflection and transmission of ultrasonic bounded beams having an $n$ th-polynomial intensity distribution. Tran D. K. Ngoc and Walter G. Mayer (Physics Department, Georgetown University, Washington, DC 20057)

Theoretical investigations of the nonspecular reflection or transmission effects in the past have been done mainly for an incident beam assumed to have a Gaussian intensity distribution. This may not be satisfactory in many cases where a beam is characterized by a non-Gaussian profile. This paper describes nonspecular effects for an incident beam whose intensity distribution is modeled by a general $n$th polynomial, providing more flexibility in simulating reflection and transmission phenomena for bounded beams. An extension of the spectral representation of bounded beams leads to calculations of reflected beam profiles at critical angles associated with a solid-liquid interface for an $\boldsymbol{n}$ th polynomial incident beam. Sample nonspecular reflection and transmission profiles are calculated for a more complex layered media system, i.e., a solid plate immersed in a liquid. Results are compared with known nonspecular characteristics associated with Gaussian incident beams. [Work supported by Office of Naval Research, U.S. Navy.]

\section{$10: 35$}

AAA7. Borehole acoustic reflection coefficients at a horizontal interface. S. K. Chang (Schlumberger-Doll Research, Box 307, Ridgefield, CO 06877)

This paper describes results of borehole acoustic reflection coefficients at a horizontal interface obtained from the finite element method (FEM). A fluid filled borehole perpendicular to the plane interface between two adjacent solid formations is considered. The reflection coefficients of compressional and shear head waves and the Stoneley mode at the boundary are obtained quantitatively. From the study of varying formation parameters and frequencies, we have observed that the compressional and shear head wave reflection coefficients are the same as those of compressional and shear plane waves normally incident on a plane boundary, as if the borehole were not present. The reflection coefficient of the Stoneley mode is dispersive and has a phase shift. The asymptotic Stoneley wave reflection coefficient can be derived by assuming no mode conversions. It was found that the asymptotic Stoneley reflection coefficient is off by a factor which is caused by mode conversions at the edge. We thus define a "mode conversion factor" to be the ratio of FEM to asymptotic results. The mode conversion factor is monotonically decreasing as frequency increases. It is approaching unity at low frequencies, indicating the dominance of Stoneley wave and the validity of the asymptotic reflection coeffcient.

\section{0:50}

AAA8. Approximate expressions for on-axis pressure of piston source radiation transmitted through a liquid-solid interface. $R$. B. Thompson and T. A. Gray (Ames Laboratory, Iowa State University, Ames, IA 50011)

The fields of a piston source in a baffle, radiating into a half-space, are well known. Approximate analytical expressions are presented which extend these to the cases of transmission through planar or cylindrically curved interfaces at normal or oblique incidence. Included are results for both the cases of longitudinal wave and transverse wave generation. In order to obtain analytically tractable results for these complex interactions, it is necessary to make a number of simplifying assumptions, such as the neglect of the variation of interface transmission coefficients over the range of angles of incidence contained within the beam. Hence, application of the results are restricted to illumination away from critical angles. Comparison to experimental measurements of scattering from on-axis reflectors are presented. The results are interpreted in terms of the change in beam cross section from circular to elliptical due to the refraction induced change in propagation direction, coupled with the focusing effects of the cylindrical surface. [Work sponsored by Center for Advanced NDE, operated by Ames Laboratory, USDOE, for AFWAL and DARPA under Contract No. W-7405-ENG-82 with Iowa State University.]

\section{1:05}

AAA9. Scattering of acoustic waves by submerged objects in the presence of a nearby boundary. V. V. Varadan, V. K. Varadan (Wave Propagation Group, The Ohio State University, Columbus, OH 43210), and L. Flax (Code 790, NCSC, Panama City, FL 32407)

The scattering of acoustic waves by objects submerged in water just above a rigid planar boundary is studied. The effect of multiple scattering between the planar boundary and the scatterer of general shape is completely accounted for. Our approach involves the use of the half-space Green's function with Neumann boundary conditions prescribed on the 
rigid planar boundary and the use of the $T$ matrix of the single scatterer. Invoking the Helmholtz integral representation for the fluid half-space, the problem is changed to an equivalent scattering configuration which is equivalent to using the method of images. Frequency dependence and the angular variation of the sound field scattered by such a configuration is studied as a function of the shape and distance from the rigid boundary of the submerged object.

AAA10. Measurements of ultrasonic scattering from flaws near a liquidsolid interface. D. K. Hsu (Physics Department, Colorado State University, Fort Collins, CO 80523), T. A. Gray, and R. B. Thompson (Ames Laboratory, lowa State University, Ames, IA 50011)

Experimental measurements of the ultrasonic scattering from voids and inclusions near a liquid-solid interface are reported. Measurements were made in a pulse-echo mode, using a transducer immersed in the liquid medium. Scatterers in the solid included an oblate spheroidal void, well removed from the interface, and spherical and prolate spheroidal inclusions, on the order of a scatterer radius away from the interface. The results are interpreted in terms of newly developed theoretical calculations for subsurface flaws [V. V. Varadan, V. K. Varadan, T. A. K. Pillai, R. B. Thompson, and D. Hsu, J. Acoust. Soc. Am. Suppl. 1 71, 82 (1982)]. Comparisons of the absolute scattering amplitudes are made in terms of frequency, flaw-to-surface distance, ultrasonic mode, and scattering angle. For the particular parameters of the near surface case studied in this work, spectral variations of the signal are dominated by the interferences of $L \rightarrow L, L \rightarrow T$, and $T \rightarrow T$ scattering from the flaw rather than multiple scattering between the flaw and surface. However, this is not viewed as a general conclusion, and a simple estimate of the magnitude of the multiple scattering process is made. [Work supported by Center for Advanced NDE, operated by Ames Laboratory, USDOE, for AFWAL and DARPA under contract No. W-7405-ENG-82 with Iowa State University.]

\section{1:35}

AAA11. Sound reflection from a fluid-loaded and masked elastic plate. $P$. D. Jackins and G. C. Gaunaurd (Naval Surface Weapons Center, White Oak, Silver Spring, MD 20910)
We have extended available computer codes that predict the classical reflections and transmissions of sound fields through sets of plane elastic layers [i.e., D. Folds and C. Loggins, J. Acoust. Soc. Am. 62, 1102-1115 (1977)]. The present layers separate possibly dissimilar fluids, are possibly viscoelastic, and are insonified from one side. The results we display include reflection $(R)$ and transmission $(T)$ coefficients as functions of frequency $f$ and incidence angles $\theta$. This computational tool has been used to predict the reflections from a single fluid-loaded elastic plate ideally covered with a lossy layer of homogeneous viscoelastic material, insonified from the coated side. Resonances in the coefficients are analyzed in the light of the resonance scattering theory (RST), which has not yet been applied to coated plates. The present model serves to assess the effectiveness of the masking layer to reduce the reflections from the metal plate The predicted displays of calculations are presented for values of the material parameters in the ranges commonly found in practice. The lossy nature of the absorbing layer complicates the otherwise real parameters present in the analysis, and we quantitatively determine the shifts in resonance locations, the widening of the resonance peaks, and the overal reduction of the returned reflections caused by the masker's viscoelasticity.

11:50

AAA12. Acoustic plane-wave scattering by a thin rigid plate with a twodimensional lattice of rectangular apertures. A. Sezginer and $S$. K. Chang (Schlumberger-Doll Research, Box 307, Ridgefield, CO 06877)

Scattering of acoustic plane waves by a thin rigid plate with a twodimensional lattice of rectangular apertures is solved by a numerical technique that identically satisfies the energy conservation. All fields are expressed as summations of Bloch waves using Floquet's theorem. The normal component of the velocity field in the apertures is expanded in a set of basis functions that have the square root singularity at the edges of the apertures, as dictated by the edge condition. The coefficients of the Bloch waves are expressed in terms of the coefficients of the basis functions, which are in turn solved using Galerkin's method. Reciprocity principle is satisfied to the desired accuracy by taking sufficient number of basis functions and Floquet terms. Wood's anomalies are observed for some Rayleigh critical angles.

FRIDAY MORNING, 12 NOVEMBER 1982

ORANGE ROOM, 9:00 A.M. TO 12:05 P.M.

\title{
Session BBB. Speech Communication VII: Phonetic Application to Languages
}

\author{
Victor W. Zue, Chairman
}

\author{
Massachusetts Institute of Technology, 77 Massachusetts Avenue, Cambridge, Massachusetts 02139
}

\author{
Chairman's Introduction-9:00
}

\section{Contributed Papers}

\section{9:05}

BBB1. Reversed sonority in Pashto initial clusters. Alan Bell and Mohamed M. Saka (Department of Linguistics, Box 295, University of Colorado, Boulder, CO 80309)

Pashto possesses both resonant clusters of rising sonority such as lwand clusters of reversed sonority, such as wl-. The highly preferred status of the first clusters has been incorporated in the syllabic theories of Bybee Hooper, Vennemann, Fujimura and Lovins, and Kiparsky. The question arises whether the dispreferred clusters manifest phonetic adaptations to compensate for their reversed sonority. The clusters ml-, lm-, lw-, wl-, and wr- and the singlet initials $\mathrm{m}-, 1-, \mathrm{r}-$, and $\mathrm{w}$ - were analyzed. Waveforms and LPC spectral analyses were obtained for 120 tokens recorded by the second author, using the Haskins Laboratories speech analysis programs. No frication was observed for any onset. All onsets were partially devoiced. The devoicing duration depended only upon the initial segment, not the sonority type of the cluster it began. Comparing the segment durations of the preferred $\mathrm{ml}-$ and $\mathrm{lw}$ - clusters to the reversed $\mathrm{lm}$ - and wl- clusters showed that the more sonorous segments were relatively longer beginning reversed clusters than ending preferred ones; the less sonorous segments 


\section{$9: 20$}

BBB2. Vowel reduction, acoustic system contrast, and vocal tract length. Florina J. Koopmans-van Beinum (Institute of Phonetic Sciences, University of Amsterdam, The Netherlands)

This paper reports on systematics in vowel systems and their relation to the speech production mechanism. First we studied the reduction of contrasts in vowel systems in several speech conditions. In order to compare the degree of acoustic system contrast of various speakers, we introduced the parameter ASC being the dispersion or total variance of a vowel system. Vowel identification tests with excised vowels revealed that on account of the measured ASC-yalues predictions can be made concerning vowel identifiability [measured identifiability for (Dutch) vowels in isolation: $90 \%$; vowels in isolated words: $84 \%$; unstressed vowels from free conversation: $33 \%$ ]. Further research showed that contrast reduction is independent of position or number of vowels in the vowel system. The reference point for calculating ASC existed in the grand mean of all measured formant frequencies per speaker. Independent of language or speech condition these speaker centroids cluster strikingly in the formant field, suggesting a close relation with vocal tract lengths.

\section{9:35}

BBB3. Fitting long rowels and stops into the mora in Japanese. Robert F. Port (Indiana University and Bell Laboratories, Murray Hill, NJ 07974) and Jonathan Dalby (Indiana University, Bloomington, IN 47405)

Earlier studies of two syllable words demonstrate that segment durations in words are contextually adjusted so as to yield word durations that are nearly equal despite differences in the inherent durations of the vowels and consonants that make up the words. The present experiment compares the temporal structure of a pair of two-mora $\mathrm{CVCV}$ words to that of three pairs of three-mora words of three different types; a pair that has an "extra" vocalic mora (CVVCV), a pair that adds a consonantal mora (CVCCV), and a third pair that adds a syllabic mora (CVCVCV). Within each pair the medial stop differs in voicing. The results show that adjustments are made between adjacent morae, not just between adjacent segments, to compensate for inherent differences in mora durations. This problem arises since a segmental mora (i.e., long $V$ or $C$ ) is shorter than a syllabic mora $(\mathrm{CV})$, yet the durations of all three words containing them were found to be nearly equal. Furthermore, the adjustments at the mora level are made independently of the effects due to adjustments at the segment level. [Work supported by NIH.]

\section{9:50}

BBB4. Some acoustic aspects of early imitation of speech: $F 0$ and vowels. Philip Lieberman, John Ryalls, and Steve Rabson (Department of Linguistics, Brown University, Providence, RI 02912)

A Japanese mother and her infant son were recorded on high quality equipment in the home setting. The imitative utterances of the infant were analyzed acoustically and compared to the mother's. The intonation contours from age five weeks were found to reproduce acoustic characteristics of $F 0$ frequency, pattern, and duration; apparently in that order of acquisition. It was also found that the mother raised her $F 0$ from an average of $185 \mathrm{~Hz}$ to an average of $325 \mathrm{~Hz}$ when interacting with her child. 325 $\mathrm{Hz}$ is within the infant's own $F 0$ range, apparently facilitating imitation. At approximately 11 weeks the child also began to spontaneously produce vowel sounds, and to imitate his mother's vowels. The infant vowels were frequency scaled, and not simple acoustic matches. The child could have reproduced some of his mother's vowels almost exactly, but instead finds a phonetic equivalence in an acoustic vowel space that is appropriate to his smaller supralaryngeal vocal tract. [Work supported by NICHHD.]
BBB5. An electropalatographic and acoustical study on coarticulation for alveopalatals $[\kappa],[\mathrm{n}]$. Daniel Recasens (Department of Linguistics, University of Connecticut, Storrs, CT 06268 and Haskins Laboratories, 270 Crown Street, New Haven, CT 06510)

Electropalatographic and acoustical data on VCV́ coarticulation for the alveopalatals $[K],[n]$ versus consonants of different place have been collected, mainly for one Catalan speaker. For alveopalatals, EPG data show almost no effect of vocalic context over time or on the point of palatal maximum constriction; consistently, corresponding acoustical measurements indicate small or no transconsonantal effects and a small range of variation for $F 2$ frequency values at the closure period for different vocalic environments. On the other hand, palatographic and acoustical data on other consonants, e.g., alveolars, show large transconsonantal coarticulatory effects. These results allow for two different articulatory preprogramming strategies largely dependent on constraints imposed by the articulators, one appropriate to a highly invariant vocal tract configuration shaped by a tongue dorsum raising gesture towards the alveopalatal area and another one appropriate to a more variable configuration subject to coarticulation with the surrounding vocalic environment.

\section{$10: 20$}

BBB6. Differences among retroflex consonants. Peter Ladefoged (Phonetics Lab, Linguistics Department, UCLA, Los Angeles, CA 90024) and $P$. Bhaskararao (Deccan College, Pune, India)

Both Dravidian languages such as Telugu and Indo-Aryan languages such as Hindi have contrasts between dental and retroflex consonants. But what is called retroflex in Telugu is not the same as what is called retroflex in Hindi. $\mathrm{X}$-rays of five speakers of each of these languages producing minimal pairs exhibiting these contrasts show that the tongue tip is curled further up and back in the Telugu retroflex consonants than in those in Hindi. The dental consonants in the two languages are more similar. The articulatory differences are reflected in the acoustic data. As expected the formant transitions for the Telugu retroflex consonants involve a greater lowering of the third formant. These results appear to be contrary to the predictions of the quantal theory of speech production, which suggests that there should not be small but significant variations in the articulation of such consonants.

\section{0:35}

BBB7. Spanish intonation in declarative sentences. Angela Signorini (Laboratorio de Fonética Experimental, Universidad Católica Argentina, Bartolomé Mitre 1869, 1039 Buenos Aires, Argentina)

The present paper attempts to describe the overall intonation pattern of Spanish declarative sentences. The global $F 0$ movements were quantified in the $F O$ curves. The starting point, the peaks and final point values were obtained from the curves. Results showed that all the sentences under study could be sorted into at least three different forms. Form I showed a raising-falling movement with only one pitch accent. This generally occurred in the shortest statements. Form II showed two pitch accents, the first in the raising slope and the second in the falling. The body, between the pitch accents, remained quite stable. Form III resembled a concatenation of two type I forms. The falling slope of the first form is not as low as the final falling slope. Depending on the criterion followed for the interpretation of this dent and the intonation unit adopted for segmentation, form III will be considered as a series of two forms I or as a form II. In the latter case, the $F 0$ movement in the body would not be relevant for the intonation pattern and could be considered the acoustic correlate of the prosodic feature boundary.

\section{$10: 50$}

BBB8, Relationship between stress and $F 0$ in Spanish. María Ignacia Massone (Laboratorio de Fonética Experimental, Universidad Católica Argentina, Bartolomé Mitre 1869, 1039 Buenos Aires, Argentina) 
The relationship between stress and $F 0$ as manifested by the $F 0$ curve in declarative and interrogative Spanish sentences is quantified. The material under study were the $F 0$ curves of 20 declarative sentences and four yes-no questions. The peaks and changes of $F 0$ of stressed syllables were measured on the $F 0$ curves and the duration of each segment on spectrograms. Three levels of stress were determined on several key content words. In declarative sentences the high level is manifested by the $F 0$ maximum: $138.42 \mathrm{~Hz}$, and by the greatest $F 0$ change with respect to the preceding syllable: $30.42 \mathrm{~Hz}$. The middle level presents the second $F 0$ peak: $124.5 \mathrm{~Hz}$ and a smaller change: $26.5 \mathrm{~Hz}$. The low level is marked by a greater vowel and consonant duration. The yes-no questions show a greater $F 0$ change and a higher overall frequency of the $F 0$ curve with respect to statements. Therefore, the pattern of interrogative sentences cue distinguishes a question from a statement. Results suggest that stress is marked by different cues according to the level of stress of the content words and to its position within the $F O$ curve.

\section{1:05}

BBB9. Final-word lengthening in Spanish. A. M. B. de Manrique (Laboratorio de Fonética Experimental, Universidad Católica Argentina, Bartolomé Mitre 1869, 1039 Buenos Aires, Argentina)

In an ongoing work about segmental duration and rhythm in Spanish it was observed that not only final vowels were lengthened but also many final-word segments seemed to be elongated. The present paper was undertaken in order to quantify this effect. A set of words which occurred in utterance final position, in utterance nonfinal position, and prior to a major constituent boundary were chosen. All syllables were open, bearing the penult syllable the stress. Results showed that the elongation was of different magnitude across the final-word segments. The greater increment was observed in the final-syllable vowel (mean final-nonfinal ratio: 2.03). The lengthening of the penult syllable may be attributed to the stress, to the positional condition or to both factors. Results showed that penult-syllable elongation was due to a positional factor in the consonant case and to both factors, stress and position, in the vowel case. It was also observed that preboundary words were longer than nonfinal but somewhat shorter than final words. Preboundary words also showed various degrees of lengthening.

\section{$11: 20$}

BBB10. An inverse filtering study of Burmese creaky voice. Hector R. Javkin and Ian Maddieson (Phonetics Laboratory, Linguistics Department, UCLA, Los Angeles, CA 90024)

A systematic study of the glottal characteristics of creaky voice in a language which uses it for linguistic contrast has not yet been done. In Burmese, syllables can contrast plain and creaky phonation. The characteristics of these phonation types were studied using inverse filtering [R. L. Miller, J. Acoust. Soc. Am. 31, 667-677 (1959); G. Fant, Q. Prog. Status Rep. Speech Transmission Lab. No. 1, 85-107 (1979)]. A Burmese speaker was recorded in a sound-treated booth using a high-quality condenser microphone and an FM tape recorder. The recording was analyzed through an analog multi-formant inverse filter. An Oscillomink was used to graph (1) the speech wave, (2) the output of the inverse filter (representing the differentiated glottal flow), and (3) the inverse filter output integrated to represent glottal flow. The differences between creaky and normal phonation can be described in terms of peak flow, the asymmetry between the rate of rise and fall of flow in each glottal pulse, as well as $F 0$. [Work supported by NIH.]

\section{1:35}

BBB11. Acoustic cues for vowel nasalization: A simulation study. Shinji Maeda (Centre National d'Etudes des Telecommunications, 22301 Lannion, France)

The manner in which vowel spectra are modified as the degree of the nasal coupling is varied was investigated by means of a simulation of the vocal-tract system. Connecting a side cavity, which represents the nasal sinuses, to the main nasal tract, speech synthesis by simulation could produce naturally sounding nasalized vowels [S. Maeda, "The role of the sinus cavities in the production of nasal vowels," Proc. IEEE ICASSP82, Paris, 911-914 (1982)]. Such spectral variations were studied in detail to shed some light on acoustic cues for vowel nasalization. The advantage of the simulation approach is that the acoustic characteristics of the nasalized vowels can be calculated for arbitrary coupling magnitude, with a realistic vocal-tract model. The computed vocal-tract transfer functions were then converted to pseudo-auditory excitation patterns. A consistent effect of the coupling, for the 11 French vowels that were investigated, was a flattening of the excitation patterns, extending in the frequency range from 300 to $2500 \mathrm{~Hz}$. Since those vowels with flattened spectra were actually heard as nasalized, it may be suggested that the principal cue for nasalization is spectral flattening in that wide frequency range.

\section{1:50}

BBB12. Monitoring speech production in a foreign language. James Emil Flege (Biocommunication, University of Alabama, Birmingham, AL 35294)

This study examined production by native and non-native speakers of French "tu" (ty) and "tous" (tu) in the absolute-initial position of phrases that: (1) were read from a list; (2) initiated spontaneously generated sentences; and (3) initiated sentences in a spontaneous story. If paying attention to speech improves performance, production should be more nativelike in condition 1 than 3 . However, no effect of speaking condition was observed in a listening test in which "tu" and "tous" were presented in pairs to French-speaking Americans. Higher correct recognition rates were observed for native French than American speakers, and for advanced compared to intermediate Americans. Labeling by native French listeners also indicated much better performance by native than non-native speakers. Recognition rates were higher for the advanced than intermediate Americans for "tous" but not "tu", with a tendency for better recognition in condition 1 than 3 for "tous" produced by both groups of A mericans. Examination of $F 2$ showed no overlap between $/ y /$ and $/ u /$ for vowels produced by the native speakers; little overlap for the advanced Americans; and nearly complete overlap between the $/ u /$ and $/ y /$ produced by the intermediate Americans due to the high $F 2$ of their $/ \mathrm{u} /$. 


\title{
Session CCC. Speech Communication VIII: Articulation and Varia
}

\author{
J. M. Baker, Chairman \\ Dragon Systems, 173 Highland Street, West Newton, Massachusetts 02615
}

Chairman's Introduction-1:30

\section{Contributed Papers}

CCC1. The funefional specificity of articulatory control and coardination. J. A. Scott Kelso (Haskins Laboratories, 270 Crown Street, New Haven, CT 06510 and University of Connecticut, Storrs, CT 06268), Betty Tuller (New York University Medical Center and Haskins Laboratories, 270 Crown Street, New Haven, CT 06510), and Carol A. Fowler (Dartmouth College, Hanover, NH 03755 and Haskins Laboratories, 270 Crown Street, New Haven, CT 06510)

Favored interpretations of "compensatory" movements of the lips to unanticipated resistances applied to the jaw during a closing gesture have focused on afferent feedback control [e.g., Folkins and Abbs (1975)] or feedforward, open-loop control processes [e.g., Abbs and Cole (1982)]. The present experiments sought to identify and analyze, using dynamic perturbation techniques, functional constraints among several articulators when they cooperate to produce a desired utterance. A brushless DC torque motor coupled to the subject's lower teeth via a custom-made prosthesis was programmed to inject a $500-\mathrm{g}$ torque load to the jaw for 1.5 s during the vowel-consonant transition in /baeb/and/baez/ on 20\%$25 \%$ of trials. Infrared L.E.D.s were attached to the lips and jaw and monitored using a modified SELSPOT optical tracking system. In experiment 1 , increased downward displacement of the upper lip occurred in perturbed conditions for $/ b /(p<0.01)$ but not for $/ 2 /$. Experiment 2 replicated this result and also showed, through hooked wire electrode recordings from a tongue muscle, a significant increase in electromyographic activity for $/ z /$ prior to occlusion, but not for $/ b /$. Closure and frication were attained on all trials without any obvious changes in timing. These highly distinctive patterns suggest that the jaw, lips, and tongue may be controlled and coordinated as a single, functional unit (a coordinative structure) that is unique and spocific to the intended act. [Work supported by NINCDS and BRSG.]

\section{1:50}

CCC2. On the kinematics of articulatory control as a function of stress and rate. Betty Tuller (Haskins Laboratories, 270 Crown Street, New Haven, CT 06510 and New York University Medical Center), J. A. Scott Kelso (Haskins Laboratories, 270 Crown Street, New Haven, CT 06510 and University of Connecticut, Storrs, CT 06268), and Katherine S. Harris (The Graduate School, City University of New York and Haskins Laboratories, 270 Crown Street, New Haven, CT 06510)

In a previous paper that analyzed phase relations among various muscles as a function of speaking rate and syllable stress [Tuller, Kelso, and Harris (1982)], we found that the time of onset of consonant-related activity remained fixed relative to time of onset of activity for flanking vowels, although substantial changes occurred in peak EMG and duration of EMG in individual muscles. This finding suggests that it is the internal timing relations that are preserved invariant across suprasegmental variation. It is not known whether the kinematic structure of the movement trajectories exhibits a pattern that can be interpreted in an analogous way. To this end, we examined subject's productions of b-vowel-consonantvowel-b utterances where the vowels were either $/ a /$ or $/ a /$ and the medial consonant either $/ \mathrm{b}, \mathrm{p}, \mathrm{v} /$ or $/ \mathrm{w} /$. Two speaking rates and two stress patterns were varied orthogonally. Infrared L.E.D.s were attached to the lips and jaw and monitored, using a modified SELSPOT optical tracking system. Thus far, the kinematics map rather well onto the earlier EMG findings and together provide evidence for relational invariance in articulation. [Work supported by NINCDS and BRSG.]
CCC3. Variability of tongue muscle activities and its implications. Kiyoshi Honda, Thomas Baer (Haskins Laboratories, 270 Crown Street, New Haven, CT 06510), and Peter J. Alfonso (Department of Communication Sciences, University of Connecticut, U-85, Storrs, CT 06268 and Haskins Laboratories, 270 Crown Street, New Haven, CT 06510)

We have collected a large amount of simultaneously recorded tongue EMG data [P. J. Alfonso et al., J. Acoust. Soc. Am. Suppl. 1 71, S54 (1982)]. The study reported here is based on these data and further investigates the coordination of the tongue muscles in static and dynamic conditions during vowel production. We examine the variability of the data associated with $F 0$ change. We also examine the intercorrelations among these muscles in terms of token-to-token EMG variability. Since the tongue consists of a network of muscles, an articulatory gesture uses 00 ordinated activities of multiple muscles. In repetitions of tokens, relative activities of the muscles vary with each other. These variabilities provide a view on the organization of muscular control of the tongue. The preliminary analysis demonstrated that when the genioglossus showed increased activity during high-pitched $/ \mathrm{i} /$, the antagonistic muscles (the hyoglossus and the styloglossus) showed some increased activities while the agonistic muscles (the mylohyoid and the geniohyoid) sometimes showed relatively low activities. This implies that these variabilities are regulated in order to maintain the noncompressive approximation of the tongue. [Research supported by NINCDS.]

\section{2:20}

CCC4. Development of lingual control. David J. Ostry and Rosemary F. Feltham (Department of Psychology, McGill University, Montreal, Quebec H3A 1BI, Canada)

In both speech and limb movements, the maximum velocity of the movement is correlated with the distance traveled. In adult lingual movements, this is accompanied by a systematic relationship between displacement and the interval from oral release to the point of maximum velocity [D. J. Ostry and A. Parush, J. Acoust. Soc. Am. Suppl. 1 71, 556 (1982)]. The development of these relationships in speech was examined in English speaking children between three and 11. The data were gathered using pulsed ultrasound to monitor tongue dorsum movements during repetitions of alternately stressed CV syllables. Findings were that displacements and maximum velocities were greater for stressed than for unstressed vowels. By age three reliable correlations were obtained between maximum velocity and displacement on both raising and lowering movements of tongue dorsum. Subjects six years or older also showed a constant interval from oral release to maximum tongue lowering velocity across differences in voicing, but like adults, estimates differed with stress. The implications of these findings to speech control and its development are discussed.

\section{2:35}

CCC5. Lingual kinematies and acoustic durations. Avraham Parush and Kevin G. Munhall (Psychology Department, McGill University, Montreal, Quebec, H3A 1B1, Canada)

In a previous paper [J. Acoust. Soc. Am. Suppl. 1 71, S105 (1982)] we showed that in $/ \mathrm{pVCV}$ / sequences, the duration of the lingual V1C tran- 
sition was inversely related to the lingual displacement for the CV 2 transition, while the displacement and maximum velocity of $\mathrm{CV} 2$ were inversely related to the displacement of VIC. Relationships among acoustic durations and these kinematic variables were examined further. The acoustic duration of VI was related to the duration of the VIC transition, while the duration of V2 was related, less consistently, to both the duration and displacement of the $\mathrm{CV} 2$ transition. The duration of $\mathrm{V} 1(\mathrm{~V} 1=/ \mathrm{a} /)$ was negatively related to the displacement of $\mathrm{CV} 2$, but the duration of $\mathrm{V} 2$ was not systematically related to the displacement of V1C. In addition, movements toward closure, relative to V1 acoustic onset, started earlier for greater V1C displacements. Movements toward V2, relative to V1 acoustic offset, started earlier for greater CV2 displacements and maximum velocities. Further, closure duration was negatively related to the displacernent and maximum velocity of the $\mathrm{CV} 2$ transition. Coproduction of vowels and consonants will be discussed.

\section{2:50}

CCC6. Stress and tongue blade movement in alveolar VC gestures. Martha Laferriere (Bell Laboratories, Murray Hill, NJ 07974)

Displacement, peak velocity, and movement time of the tongue blade in VC transitions were studied using data obtained by a computer-controlled x-ray microbeam system [cf. Laferriere and Fujimura (1981)]. Consonants were $/ t /$ and $/ d /$; vowels were either stressed, or unstressed/ reduced. Displacement was the distance moved by the blade pellet from a zero-velocity state for the vowel to the next zero-velocity state for the consonant. A positive relationship was found between displacement and velocity [cf. Kuehn and Moll (1976)]. However, when C was a stop, for a given displacement, blade movement was faster from an unstressed vowel than from a stressed vowel. When $\mathrm{C}$ was a tap, for a given displacement, blade movement was faster when the following vowel was unstressed

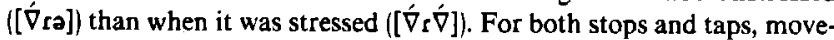
ment time was shorter in the unstressed cases. These results indicate that (1) velocity and movement time of the tongue blade are conditioned by degree of stress, and (2) the broader stress environment (post-consonantal vowel) may also affect articulatory movement of the pre-consonantal transition.

\section{3:05}

CCC7. Articulatory strategies for combining vocalic elements. Fredericka Bell-Berti (St. Johns University, Jamaica, NY 11439 and Haskins Laboratories, 270 Crown Street, New Haven, CT 06510) and Lawerence J. Raphael (Herbert H. Lehman College, CUNY, Bronx, NY 10468 and Haskins Laboratories, 270 Crown Street, New Haven, CT 06510)

Sequences of vowels in English are perceived (and, presumably, produced) variously, depending upon their linguistic functions. Thus, [i] and [?] can be combined to produce: (1) a one-syllable, one-phoneme unit such as the diphthong in boy; (2) a one-syllable, two-phoneme unit (in which the first vocalic element functions as a consonant) such as the glide-plusvowel sequence in yawn; (3) a two-syllable, two-phoneme unit such as the vowel sequences in $I$ see Audie and $I$ saw Edie. An electromyographic study of intrinsic and extrinsic muscles of the tongue has yielded a preliminary description of the articulatory strategies used to differentiate these combinations of vocalic elements. [Research supported by NINCDS.]

\section{$3: 20$}

CCC8. One-stage and two-stage temporal patterns of velar coarticulation. Ameen Al-Bamemi and Anthony Bladon (Phonetics Laboratory, University of Oxford, 41 Wellington Square, Oxford, OXI 2JF, England)

Previous observations of the coarticulatory spread of nasality from a nasal consonant to adjacent vowels have yielded some contradictory evidence and two contradictory hypotheses: (a) coarticulatory timing is determined by segmental content and (b) coarticulation is coordinated in time with a particular articulatory gesture. Using transillumination of the velopharyngeal port, the timing patterns of velum opening were investigated in $(C) V N,(C) V V N$, and (C)VVVN sequences in languages lacking contrastively nasalized vowels. It was found that all speakers used two distinct patterns of velum opening, though with different frequencies of occurrence: first, a single opening gesture of smoothly increasing amplitude, its onset aligned with the first vowel onset irrespective of intervening boundaries; and second, a two-stage opening gesture whose absolute onset was aligned as previously but whose higher-velocity second stage was coordinated with the oral closing gesture for the nasal consonant. Thus we may have to recognize that speakers have available two alternative production strategies.

\section{3:35}

CCC9. Nasal coarticulation of pharyngeal and glottal consonants: $A$ deductive account. Anthony Bladon and Ameen Al-Bamerni (Phonetics Laboratory, University of Oxford, 41 Wellington Square, Oxford, OX1 2JF, England)

The general observation [e.g., Bell-Berti, Stat. Rep. Speech Res., Haskins Labs., 46 (1980)] that velum height decreases as articulatory constriction decreases is examined in the context of pharyngeal and glottal obstruents in Arabic and Kurdish. Investigated by transillumination of the velopharyngeal port, these consonants are seen to be always produced with a substantial velopharyngeal opening. Moreover, the "nasality" exhibits a coarticulatory spread to adjacent vowels. A deductive account of consonant features could use considerations of articulatory synergy and biological bias to predict such findings, in particular the requirement that $/ \mathrm{h} \varsigma \mathrm{h} /$ should be specified as [ + nasal]. In addition, such an account might predict that for reasons of perceptual sufficiency the temporal spread of velopharyngeal opening from a pharyngeal or glottal consonant would be less extensive than from a nasal consonant. This is in fact shown to be the case.

\subsection{0}

CCC10. Laryngeal adjustments in stop and fricative production. Kevin G. Munhall, Avraham Parush, and David J. Ostry (Department of Psychology, McGill University, Montreal, Quebec, H3A 1B1, Canada)

A computerized pulsed ultrasound system was used to study the dynamics of laryngeal abduction during obstruent production. Subjects produced the consonants $/ \mathrm{s} /$ and $/ \mathrm{t} /$ in a $/ \mathrm{trC \varepsilon t} /$ context at two rates, with either the first or second vowel stressed. Preliminary results indicate that the time from offset of the first vowel to the maximum laryngeal opening was positively related to oral closure or approximation duration. However, the maximum opening of the gestures associated with the production of $/ \mathrm{s} /$ occurred closer to vowel offset than those for $/ \mathrm{t} /$ in comparable conditions. In addition, the interval from vowel offset to maximum opening velocity was found to vary as a function of stress and consonant. The time to maximum velocity was shorter for the gesture associated with $/ \mathrm{s} /$ than for $/ t /$ and shorter for consonants following a stressed vowel than for those preceding stressed vowels. These aspects of laryngeal displacement and timing will be discussed in terms of interarticulator organization.

4:05

CCC11: Abstract withdrawn.

\section{4:20}

CCC12. Some acoustic characteristics of Parkinsonian dysarthria. Gary Weismer (Speech Motor Control Laboratories, Waisman Center, University of Wisconsin-Madison, Madison, WI 53706)

The early stages of Parkinsonian dysarthria are often thought to be characterized primarily by laryngeal dysfunction, whereas in later stages a progressive deterioration of supraglottal articulatory function is observed. Most previous analyses of Parkinsonian dysarthria have employed phonetic transcription as a descriptive device, but a few recent papers [e.g., Tatsumi et al., Ann. Bull. RILP 13, 99-104 (1979); Kent and Rosenbek, Brain Lang. 15, 259-292 (1982)] have reported some more objective 
dâta based on acoustic measures. In the present study, we analyze acoustically the temporal structure of sentence recitations produced by ten Parkinsonian dysarthrics. These data are compared to existing data sets for the same utterances produced by normal young adults and geriatrics. Results indicate that the temporal characteristics of the Parkinsonian sentence recitations are only subtly different from those of young adults and geriatrics. However, the spontaneous speech of the Parkinson's patients have clearly aberrant temporal structures. The findings are discussed relative to the general pathophysiology of Parkinson's disease. [Work supported by NIH Program Project NS 13274.]

FRIDAY AFTERNOON, 12 NOVEMBER 1982

DADE AND FLORIDA KEYS ROOMS, 1:35 TO 5:10 P.M.

\author{
Session DDD. Physical Acoustics IX: Radiation and Scattering
}

\author{
Vijay K. Varadan, Chairman \\ Department of Engineering Mechanics, Ohio State University, Columbus, Ohio 43221
}

Chairman's Introduction-1:35

Contributed Papers

$1: 40$

DDD1. Focusing concentric ring electrode array transducer. Richard O. Claus, Janet C. Wade, George D. Dockery, and Kenneth B. Ocheltree (Department of Electrical Engineering, Virginia Polytechnic Institute and State University, Blacksburg, VA 24061)

We have previously described the generation of a two-dimensional Gaussian profile ultrasonic field using a piezoelectric transducer with concentric ring electrodes [R. O. Claus and P. S. Zerwekh, J. Acoust. Soc. Am. Suppl. I 71, S12 (1982)]. In this paper, two-dimensional ultrasonic field focusing achieved by a similar transducer is presented. The focusing transducer was constructed using a $7.62-\mathrm{cm}$-diam crystal quartz disk dimensioned to resonate at $500 \mathrm{kHz}$. Concentric circular electrodes 1.27 $\mathrm{mm}$ wide and separated by $1.27 \mathrm{~mm}$ spaces were deposited on one flat surface of the disk and an aluminum foil ground plane and wear plate was attached to the opposite surface using conducting epoxy. By individually varying the time delays between the $300-\mathrm{V}$ 10-ns pulses applied to the electrodes, the location of the focal plane is controlled. In this plane, focus spot half-power diameters of less than two acoustic wavelengths have been obtained. Experimental measurements obtained in a water tank using electrode phasing for both linear geometrical superposition and lenslike focusing are presented and compared with theoretical results. [Work supported in part by NASA and NSF.]

\section{1:55}

DDD2. Difference equation evaluation of the evolution of radially symmetric ultrasonic fields. George $D$. Dockery, Richard $O$. Claus, and Paul S. Zerwekh (Department of Electrical Engineering, Virginia Polytechnic Institute and State University, Blacksburg, VA 24061)

A simplified difference equation technique has been developed to calculate the three-dimensional evolution of radially symmetric complex ultrasonic fields. Localized changes in the magnitude of the field with respect to distance along the axis of propagation are first assumed to be small compared to those with respect to radial displacement. The complex Helmholtz equation in cylindrical coordinates then may be simplified by separating variables and approximating these variables by truncated series expansions. The resulting difference equation determines complex field values at discrete locations in a plane from corresponding adjacent locations in the preceding plane. Stability criteria which limit both the step sizes between consecutive planes and between calculated field values in one plane are discussed. The technique has been applied to the propaga- tion of ultrasonic fields generated by two-dimensional focusing and nonfocusing uniform and Gaussian profile piezoelectric transducers and theoretical and experimental data are presented. [Work supported in part by NASA and NSF.]

\section{2:10}

DDD3. Point-to-point impulse response due to the presence of simple reflectors. Dov Hazony and Thomas E. Kocher (Department of Electrical Engineering and Applied Physics, Case Institute of Technology, Case Western Reserve University, Cleveland, $\mathrm{OH}$ 44106)

Recently there has been considerable activity in the area of time-dependent impulse response of acoustic radiators [P. R. Stephanishen, J. Acoust. Soc. Am. 49, 1629-1638 (1971)]. A logical extension of these ideas is to the problem of acoustic radiators acting as reflectors. The impulse response for a system consisting of a simple source, a reflector, and a receiver was determined for several reflector geometries. Each reflector was analyzed with a source $\left(P_{1}\right)$ and a receiver $\left(P_{2}\right)$ for several important cases. The specific case, when $P_{1}=P_{2}$, i.e., transmit-receive, provides a very important special case. The principle tool used was Rayleigh's integral. [Work supported by NEI \#5R01EY03251-02 and USAF \#F33615-81-C-0103.]

DDD4. Geometric reconstruction of flaws by one-dimensional inverse Born approximation. D. K. Hsu (Physics Department, Colorado State University, Fort Collins, CO 80523), James H. Rose, R. B. Thompson, and D. O. Thompson (Ames Laboratory, Iowa State University, Ames, IA 50011)

We report a three-dimensional reconstruction of the size, shape, and orientation of volumetric flaws (inclusions and voids) based on the results of one-dimensional Born inversions of the scattering amplitudes for observation directions confined to a finite angular aperture. The reconstruction is realized by performing nonlinear least squares iteration of the Born inversion results in terms of an equivalent ellipsoid. We have successfully reconstructed spherical and nonspherical inclusions in the bulk of plastic samples and an oblate spheroidal void in a titanium disk. In addition we have applied the ellipsoidal reconstruction method to a spheroidlike inclusion very near the surface of a sample and obtained good results. The method was found to remain quite successful even with experimental data confined to a rather limited aperture. Effects on the one-dimensional in- 
verse Born algorithm due to characteristic resonances of the inclusions and flaw-surface interactions will be discussed. [Work sponsored by Engineering Program, Office of Basic Energy Sciences; USDOE and by Center for Advanced NDE, gpereted by Ames Laboratory, USDOE, for AFWAL and DARPA us erded tract No. W-7405-ENG-82 with Iowa State University.] $=$

\section{2:40}

DDD5. Focusing and diffraction of backscattering from fluid spheres: Comparison of the partial-wave sum with a model. Philip L. Marston and Dean S. Langley(Department of Physics, Washington State University, Pullman, WA 99164) $-\mathbf{x}-$

Consider the scattering of a plane wave from a sphere of radius a imbedded in a fluid with a sound speed $c_{0}=M c_{i}$. Backscattered rays with impact parameters $b \neq 0$ are known as glory rays; their contributions $f_{n}$ to the total normalized form function $f$ are enhanced due to axial focusing. Naive application of ray acoustics predicts that $\left|f_{n}\right| \rightarrow \infty$ as $\gamma \rightarrow 0$ where $\gamma$ is the angle relative to the backward axis. We derive an approximation which removes this divergence and describes the effect of diffraction on axial focusing. It is similar to our model for the optical glory of bubbles [D. S. Langley and P. L. Marston, Phys. Rev. Lett. 47, 213-916 (1981)] and it gives $\left|f_{n}\right|=(k a)^{1 / 2} G_{n}\left|J_{0}(k b \sin \gamma)\right|$, where $k$ is the wavenumber and $G_{n}$ is a function of the number of internal chords $n$ and of fluid properties. The modeled $f$ is obtained by interfering several $f_{n}$ with axial reflections. It was compared with an exact partial-wave sum for $f$ with $k a=100$ and 1000 and various $M<1$; our model correctly describes the main features. A strong additional enhancement of $f(\gamma=0)$ is present when $M \approx 1.180$ due to a coincidence of $n=3$ glory and rainbow rays. [Work supported by ONR. Marston is an Alfred P. Sloan Research Fellow.]

$$
\text { ?. ' }
$$

DDD6. Observation of the acoustic glory: Scattering from an elastic sphere in near bacy B. Hanson,") and Kevin L. Williams (Department of Physics, Washington State University, Pullman, WA 99164)

We have measured the scattering for small angles $\gamma$ (relative to the backward axis) from a fused silica sphere of radius $a \approx 52 \mathrm{~mm}$. Tone bursts in water corresponding to $k a \approx 450$ were incident on the sphere; their short duration permitted glory and axial returns to be separated in time. The $\gamma$ for the probe hydrophone was scanned to test a model [P. L. Marston and L. Flax, J. Acoust. Soc. Am. Suppl. 168, S81 (1980)] of diffractive effects on backward axial focusing. Observations tend to support the model as adapted to fused silica: (1) from the arrival time, the strongest echo is evidently due to the 4-chord shear glory ray; (2) its amplitude is $\propto J_{0}(k b \sin \gamma)$ where $b$ is the calculated glory circle radius; (3) its amplitude at $\gamma=0$, though slightlysmaller than predicted, exceeds that of the first axial reflection; and (4) thine times, amplitudes, and $\gamma$ dependences of other echoes are correlated to predictions. The first null of the strongest echo occurs at $\gamma \simeq 1^{\circ}$. Consequently, we demonstrate for the first tine the diffraction limited backward focusing of echoes from a sphere. [Work supported by ONR. Marston is an Alfred P. Sloan Research Fellow.] a) Present address: Defense Systems Division, Honeywell Inc., Hopkins, MN 55343.

\section{3:10}

DDD7. Pressure patterns in the nearfield of a diffracting sphere. $R$. V. Waterhouse (Code 1940, David Taylor Naval Ship R\&D Center, Bethesda, MD 20084 and Physics Department, American University, Washington, DC 20016)

A steady train of plane waves impinges on a sphere. The nearfield diffraction patterns for the normalized rms pressure were computed for values of $k a$ from $1.4 \$ 6$, where $k$ is the wavenumber and $a$ is the radius of the sphere. Charts are given, showing the contours of $\mathrm{ms}$ pressure for $k a=1.5,2,3,4$, and 6, for each of two boundary conditions, pressure reflecting and pressure release. Each chart shows the contour pattern out to a distance of ten radii. The computed expressions are infinite series containing spherical Bessel functions and their gradients. In some cases 75 terms in the series must be evaluated to get convergence to within $1 \%$. The charts show that there are substantial areas where the pressure differs from the undisturbed value by $6 \mathrm{~dB}$ or more.

\section{3:25}

DDD8. "Whispering" waves in a wine glass. Robert E. Apfel (Applied Mechanics, Yale University, New Haven, CT 06520)

The circular motion of a wet finger on the rim of a wine glass that results in the familar resonance of the glass with the accompanying radiation of a tone is also responsible for the launching of circular fajpillary waves (wavelength $\sim 1 \mathrm{~mm}$ ) on the surfitee of the liquid (wine, or other). These waves interfere to produce visible ripples that are nearly stat phary relative to the moving finger and that have the interesting feature of teing confined to a region near the outer perimeter of the glass. Such wal $s$ are analogous to the whispering galley, "aeriak, waves that Lord $\mathbf{P l}$ leigh speaks of in one of his many classic papers (Philos. Mag. 20, 1001-1004 (1910)]. The width of the confined region, which is enuch less than the radius of the glass, is accurately predicted by Rayleight formula for circular membrane waves. Moreover, because the width of the waves is small, the waves can be considered to be one dimensional—a feature that may suggest the application of this phenomenon to the study of others. Demonstration of these waves will be given during the session (as well as at dinner).

3:40

DDD9. Radiation pattern analysis of acoustical scattering from elastlc objects immersed in a fluid. M. F. Werby, L. H. Green, and L. Flax (Naval Coastal Systems Center, Panama City, FL 32407)

Resonance patterns at different aspect angles can be quite complicated and consequently difficult to analyze $\{$ pr a variety of geometrical shapes: A theoretical study based on form function calculations can be both costly, time-consuming, and subject to ambiguity for a variety of aspect angles. We argue that it is possible to perform a sequence of radiation pattern calculations that prove useful in determining resonances at various aspect angles and illustrate this procedure with a number of calculations using computer codes recently developed at NCSC.

\section{3:55}

DDD10. Resonances of elastic spheroidal bodies. L. H. Green, M. Werby, and L. Flax (Naval Coastal Systerns Center, Panama City, FL 32407)

The calculation of the resonances [L. Flax, G. C. Gaunaurd, and H. Uberall, Theory of Resonance Scattering, in Physical Acoustics, Vol. XV, edited by W. P. Mason and R. N. Thurston (Academic, New York, 1981)] of elastic bodies immersed in a fluid is a topic of current interest. In this paper the $T$-matrix approach is applied to the stat ering of plane acoustic waves incident upon spheroidal elastic bodies immersed in water. Various aspect ratios and shell thicknesses will be treated. The wavenumber of the incident wave will satisfy $5<k L / 2<12$, where $k$ is the wavenumber for the incident sound wave and $L / 2$ is the semi-major axis of the spheroidal body.

\section{4:10}

DDD11. A new hybrid T-matrix-boundary element approach for elastic shell scattering problems. $\mathrm{V}$. V. Varadan, K. Eswaran, and V. K. Varadan (Wave Propagation Group, Department of Engineering Mechanics, The Ohio State University, Columbus, $\mathrm{OH} 43210$ )

A new method is proposed and implemented for the study of acpustic wave scatteringty thin elastic shells of revolution submerged in a fiuid. Using the conventional $T$-matrix approach for layered scatterers, diffict!ties were encountered in the past as the shell thickness decreased and the eccentricity of the object increased. This has been overcome for the case of elastic shells by invoking elastic thl $d_{-}$shell theory to characte ze the response of the shell to an incident harmonic wave. This his coupled winthe $T$-matrix approach to arrive at a solution for the rolution scattering problem. Very encouraging numerical results have vict obtained forta finite cylindrical shell with spherical end caps that is im- 
mersed in water. The frequency spectrum has been interpreted by computing the free vibration frequencies of the shell and are now being compared with the experimental results of S. K. Numrich and L. Dragonette of the Naval Research Laboratory, Washington, DC.

\section{$4: 25$}

DDD12. A new $T$-matrix approach for acoustical scattering from elastic shells immersed in a fluid. M. F. Werby, L. H. Green, and L. Flax (Naval Coastal Systems Center, Panama City, FL 32407)

We derive equations using the $T$-matrix approach for acoustical scattering from elastic shells immersed in a fluid. The equations contain unitary and symmetry constraints and afford a new procedure for the calculation of the $T$-matrix. The technique, in addition to satisfying symmetry and unitary conditions, proves to be more numerically stable and less time consuming than the conventional method. In addition, the procedure enables one to determine if convergence has been satisfied and in the limit of no convergence, it proves to be a better approximation than that of the matrix method. We have developed computer codes using the new tech: nique and present comparisons with the conventional procedure. A salient feature of the new technique is that convergence is arrived at with a smaller number of expansion terms than the older method and this is particularly true as the aspect ratio of the target is increased.

\section{4:40}

DDD13. Convergence and the choice of expansion functions in the $T$ matrix approach, L. H. Green, M. Werby, and L. Flax (Naval Coastal Systems Center, Panama City, FL 32407)
In the context of electromagnetic scattering problems, Waterman [ $\mathbf{P}$. C. Waterman, Survey of $T$-Matrix Methods, in Acoustic, Electromagnetic and Elastic Wave Scattering, edited by V. K. Varadan and V. V. Varadan (Pergamon, New York, 1980)] has considered several different choices of expansion functions for the surface currents, when applying the $T$-matrix approach. We will consider the scattering of plane acoustic waves incident upon finite elastic bodies. How different choices of the expansion functions for the surface displacements affect the rate of convergence of the elements of the $T$ matrix and the form function will be presented. Both spherical and nonspherical elastic bodies will be considered.

\section{$4: 55$}

DDD14. Some numerical techniques and their use in the extension of the $T$-matrix approach to scattering. L. Flax, R. Hackman, and $M$ F. Werby (Naval Coastal Systems Center, Panama City, FL 32407)

The $T$-matrix methodology of Waterman has been used to describe acoustic, electromagnetic, and elastic scattering. Some of the limitations on this method from a calculational point of view have been due to difficulties in treating large matrices which arise in association with the need for large sets of basic states required for the description of surfaces which deviate from classical symmetries as well as for high-frequency calculations. We present a number of useful numerical techniques for large, illconditioned matrices which improve the range of applicability of the $T$. matrix theory.

\title{
Session EEE. Psychological Acoustics VII: Testing and Conserving Hearing
}

\author{
Larry E. Humes, Chairman
}

Division of Hearing and Speech Sciences, Vanderbilt University School of Medicine, Nashville, Tennessee 37232

Chairman's Introduction-2:00

\section{Contributed Papers}

2:05

EEE1. Extended high tone hearing performance $(0.25$ to $20 \mathrm{kHz})$ in orchestral musicians with unremarkable hearing health histories. David W. Johnson and Jeffrey H. Aldridge (Department of Otolaryngology, University of Minnesota Medical School, Minneapolis, MN 55455), Robert E. Sherman (Office of Planning and Development, Hennepin County Government Center, Minneapolis, MN 55487), and Adele Lorraine (Minnesota Orchestra, Minneapolis, MN 55403)

Thirty-four members of the Minnesota Orchestra were evaluated otologically and audiologically for hearing acuity for the frequency range 0.25 to $20 \mathrm{kHz}$. Members were grouped by age and sex. Median hearing thresholds for each group for each frequency were calculated and compared to currently available presbycusis tables for the frequencies 0.25 to 8 $\mathrm{kHz}$ and to statistically generated age/sex thresholds for the frequencies 9 to $20 \mathrm{kHz}$. An analysis of individual and median audiometric configurations for each age/sex group explored potential relationships between instrument and position in the orchestra and audiogram. Generally, median performance curves showed a "noise notch" at $6 \mathrm{kHz}$ and decrease of hearing acuity with age for all frequencies, but decrease was most dramatic in the extended high tones ( 9 to $20 \mathrm{kHz}$ ). Certain musician types did demonstrate greater characteristics of noise induced hearing loss. [Work Supported by the Minnesota Foundation for Acoustical Education and Research.]
EEE2. Implications of a population study of hearing thresholds and noise exposure. S. Gatehouse (MRC Institute of Hearing Research-Scottish Section, Southern General Hospital, Glasgow, Scotland), A. C. Davis, and M. P. Haggard (MRC Institute of Hearing Research, Nottingham, England

The Institute of Hearing Research is currently undertaking a 4-yr 3phase national study of hearing in the UK. The multicenter team is applying a comprehensive clinical and audiological evaluation to a stratified sample of approximately 2400 individuals drawn from a questionnaire sample of 36000 . This permits linkage of data on impairment, pathology, and disability to population prevalence. The data furnish many results; two are reported here. Data from equivalent noise exposure levels of $<80$ $\mathrm{dB}(\mathrm{A}), 81-90 \mathrm{~dB}(\mathrm{~A})$ and $>90 \mathrm{~dB}(\mathrm{~A})$ show significant effects of noise exposure at $2000 \mathrm{~Hz}$ and above for the highest exposure band, but not for the midexposure band. This implies that the concept of socioacousis, where prolonged exposure to relatively low levels of noise causes a hearing deficit, may not be valid. There is an interaction with age and a consistent dip in the thresholds at $6000 \mathrm{~Hz}$. The hearing levels of young atologically screened subjects deviate systematically from the ISO standard for audiometer calibration and again exhibit a discernible dip at $6000 \mathrm{~Hz}$. These data throw some doubt on the ISO standard, particularly at $6000 \mathrm{~Hz}$. 
EEE3. Reference equivalent threshold sound pressure levels at 5 and 6.3 kHz using telephonics TDH 39 earphones with MX-41/AR cushions. D. A. Benwell and R. G. Hussey (Radiation Protection Bureau, Health and Welfare Canada, Room 233, Environmental Health Centre, Tunney's Pasture, Ottawa, Ontario, K1A 0L2, Canada)

Reference equivalent threshold sound pressure levels (RETSPLs), were measured at 5 and $6.3 \mathrm{kHz}$ using Telephonics TDH 39 earphones with MX-41/AR cushions. The purpose of the test was to verify RETSPL values calculated by interpolation from those specified in present International Standards Organization acoustics standards, ISO 389 "Standard reference zero for the calibration of pure-tone audiometers," and its amendments. The RETSPL values are intended for the calibration of audiometers using pure tones of fixed frequencies at the preferred frequencies in one-third octave steps or pure-tone audiometers having a continuously variable frequency. Mean measured values of RETSPL's at 5 and $6.3 \mathrm{kHz}$ were $10.9 \pm 2.6$ and $15.1 \pm 2.4 \mathrm{~dB}$, in comparison to predicted values which were 13 and $15 \mathrm{~dB}$, respectively.

\section{2:50}

EEE4. Impact on telephone operator word recognition performance of headphone type and background noise level. David W. Johnson and Robert E. Sherman (Hennepin County Medical Center, 701 Park Avenue, Minneapolis, MN 55415)

Twenty switchboard operators with clinically normal hearing were tested for ability to recognize W-22 words presented to control for order and sequence effects in six approximately matched 25 -word lists. Tape lists were dubbed from an earlier generation of taped lists where signal-to(cafeteria)-noise ratios were 0 to $+15 \mathrm{~dB}$. Lists were presented with operators wearing their own headsets (e.g., Plantronics Star Set) and the newly introduced Danavox Stetomike (which allowed either monaural or binaural listening). Testing performed in a standard double-wall construction sound booth allowed testing each headset configuration (i.e., their own monaural headset, the Stetomike monaural headset, and the Stetomike binaural headset) in quiet and in background noise approximating an actual listening environment faced by operators (a tape of actual operator room noise presented at $+55 \mathrm{dBA}$ ). There was great individual difference in the subjects' ability to correctly recognize words with older subjects performing significantly poorer than younger and the background noise greatly impairing performance overall. There was no statistically significant difference in performance which could be attributed to headphone configuration in either quiet or noisy background conditions.

\section{3:05}

EEE5. A psychophysical evaluation of the dependence of hearing protector attenuation on noise level. Larry $E$. Humes and Jayne B. Ahlstrom (Division of Hearing and Speech Sciences, Vanderbilt University School of Medicine, Nashville, TN 37232)

The attenuation characteristics of ten commercially available hearing protectors (five muffs and five plugs) were examined for one-third octavebands of noise centered at 1000,2000,3150,4000, and $6300 \mathrm{~Hz}$ in ten normal-hearing young adults utilizing four psychophysical procedures. One of these procedures, the real-ear attenuation at threshold procedure described in the American standard for the evaluation of hearing protectors, is designed for use at one-third octave band noise levels below approximately $50 \mathrm{~dB}$ SPL. Three new real-ear psychophysical procedures were also developed and evaluated. These procedures were: (1) a reactiontime paradigm; (2) a loudness magnitude-estimation procedure; and (3) a masked bone-conduction threshold technique. Each procedure was designed to evaluate protectors at one-third octave band noise levels ranging from approximately 50 to $90 \mathrm{~dB}$ SPL. Mean data indicated that: (1) attenuation was linear over the range investigated, although some exceptions to this generalization were apparent; $(2)$ attenuation estimates derived with the reaction-time and magnitude-estimation paradigms were typically lower than those obtained with the other two methods; and (3) all attenuation estimates, regardless of procedure, were lower than manufacturer's specifications for the majority of the protectors. Implications for existing standards are discussed.
EEE6. Perceptual errors arising from the use of multiband nonlinear hearing aids. Harvey Dillon, Gary Walker, and Denis Byrne (National Acoustic Laboratories, 5 Hickson Road, Sydney, 2000, Australia)

Tests were performed of the intelligibility of nonsense monosyllables processed by a computer-simulated multiband hearing aid incorporating compression for high level signals and expansion for low level signals in each band. The overall results indicated that the nonlinear system led to slightly better intelligibility for some subjects at some presentation levels, but otherwise led to equal performance or was even disadvantageous. A traditional examination of the confusions (place/manner, initial/final, vowel context) only revealed that all such subclasses were affected to a similar extent. Examination of the responses to individual items, however, revealed that the perception of a considerable number of items was made consistently better by the processing, while that of others was made consistently worse. Acoustical analysis of these items before and after processing indicated that band-edge effects (which modified formant transitions), and the time dependent frequency response (which either enhanced or degraded the characteristic spectral shapes of bursts and fricatives) were responsible for many of the changes in perception. A processing scheme which uses parametric serial filtering and cross-band gain control signals, which may overcome these problems, has been developed.

\section{3:35}

EEE7. Optimizing modulation rate and waveform for frequencymodulated sound field audiometric stimuli. Harvey Dillon and Gary Walker(National Acoustic Laboratories, 5 Hickson Road, Sydney 2000 , Australia)

Narrow-band stimuli used for audiological sound field testing should satisfy three conditions: (1) have a uniform distribution of power within the band; (2) have a rapid falloff of power outside the band; (3) have amplitude fluctuation (resulting from the rooms transmission response) which are fast enough to be fully integrated by the ear. The first condition ensures that stimuli of a given bandwidth will produce a reverberant field which is as uniform with distance as possible. For $\mathrm{fm}$ stimuli, it is well met by the use of triangular or ramp modulation waveforms. The second condition is important if people with steeply sloping hearing thresholds are not to have their hearing loss underestimated. Sinusoidal modulation waveforms met this condition well and triangular waveforms acceptably so. For the hearing impaired, with reduced temporal integration functions, the last condition is not well met by the usual $5-\mathrm{Hz}$ modulation rate. If the rate is made too high, condition ( 1 ) is violated. The optimum rate appears to be about $20 \mathrm{~Hz}$ and the optimum waveform is triangular.

\section{3:50}

EEE8. The use of a control microphone in reverberent sound field audiometric testing. Harvey Dillon (National Acoustic Laboratories, 5 Hickson Road, Sydney 2000, Australia)

Traditionally, sound field stimuli are "pre-calibrated" at a particular point in the room. Head diffraction effects are thus automatically taken into account when measurements are performed. However, the method is potentially innacurate for two reasons. First, the head diffraction effects measured are dependent on the particular test situation. Second, subjects tend to move away from the pre-calibrated point. An alternative technique is to continuously monitor the SPL in the vicinity of the subject by using a control microphone attached to the subject's head. The method has the advantage that the calibration point then moves with the subject. However, it has the disadvantages that the SPL at the control microphone is not the same as the SPL at the point of interest (hearing aid microphone or eardrum), and that average head diffraction effects must be assumed. Experiments were performed using probe microphones and control microphones in various locations on six adults and a manikin (KEMAR) using both calibration techniques. A quantitative examination of the sources of error in both procedures revealed the control microphone technique to be superior, especially when assessing aided hearing. 